\title{
Kationen-Ordnung in ferri/ferromagnetischen perowskitischen Dünnfilmen
}

\section{Dissertation}

zur Erlangung des mathematisch-naturwissenschaftlichen Doktorgrades

"Doctor rerum naturalium"

der Georg-August-Universität Göttingen

im Promotionsprogramm PROPHYS

der Georg-August University School of Science (GAUSS)

vorgelegt von

Sebastian Hühn

aus Erfurt

Göttingen, 2015 


\section{Betreuungsausschuss}

Prof. Dr. Vasily Moshnyaga, I. Physikalisches Institut

Prof. Dr. Christian Jooß, Institut für Materialphysik

\section{Mitglieder der Prüfungskommission}

Referent: $\quad$ Prof. Dr. Vasily Moshnyaga, I. Physikalisches Institut

Korreferent: $\quad$ Prof. Dr. Christian Jooß, Institut für Materialphysik

2. Korreferent: Prof. Dr. Johan Verbeeck, EMAT, Universität von Antwerpen

Weitere Mitglieder der Prüfungskommission

Prof. Dr. Konrad Samwer, I. Physikalisches Institut

Prof. Dr. Stefan Mathias, I. Physikalisches Institut

Prof. Dr. Ulrich Krebs, Institut für Materialphysik

Tag der mündlichen Prüfung: 




\section{Inhaltsverzeichnis}

$\begin{array}{llr}1 & \text { Einleitung } & 9\end{array}$

2 Grundlagen zur elektronischen Struktur der Perowskite 13

2.1 Einfluss der Struktur auf die orbitale Besetzung . . . . . . . . . . . . . 14

2.1.1 Die Kristallfeldaufspaltung . . . . . . . . . . . . . . . . . . 14

2.1.2 Der Jahn-Teller Effekt . . . . . . . . . . . . . . . . . . . . . . . . . . 14

2.1.3 Einfluss der Fehlanpassung zwischen Substrat und Dünnfilmen . . . . 16

2.2 Austauschmechanismen in Perowskiten . . . . . . . . . . . . . . 16

2.2.1 Der Superaustausch . . . . . . . . . . . . . . 17

2.2.2 Der Doppelaustausch . . . . . . . . . . . . . . 20

2.3 Einfluss der Gitterverzerrungen auf die Austauschwechselwirkungen . . . . . 22

3 Experimentelle Methoden $\quad 25$

3.1 Metallorganische Aerosol Deposition . . . . . . . . . . . . . . 25

3.1 .1 Grundprinzip . . . . . . . . . . . . . . . . . . 25

3.1.2 Standard MAD (MAD-1) . . . . . . . . . . . . 26

3.1.3 MAD mit in situ Wachstumskontrolle (MAD-3) . . . . . . . . . 27

3.1.4 Die Substrate . . . . . . . . . . . . . . . . . 29

3.2 Charakterisierungsmethoden . . . . . . . . . . . . . . 30

3.2.1 Rastersondenmikroskopie . . . . . . . . . . . . . . . . . . . 30

3.2.2 Röntgendiffraktion . . . . . . . . . . . . . . . . . 32

3.2 .3 Röntgenreflexion . . . . . . . . . . . . . . . . . . . 34 
Inhaltsverzeichnis

3.2.4 Transmissionselektronenmikroskopie . . . . . . . . . . . . . 35

3.2.5 Raman-Spektroskopie . . . . . . . . . . . . . . . . . 37

3.2 .6 SQUID Magnetometrie . . . . . . . . . . . . . . 37

3.2.7 Magnetooptischer Kerr-Effekt . . . . . . . . . . . . . . . . . . . . 40

3.2.8 Messung des elektrischen Widerstands . . . . . . . . . . . . . . . 40

4 A-Platz Ordnung in ferromagnetischen halbmetallischen Manganaten

4.1 Künstliche elektronische Phasenseparation in teilgeordneten LPCMO . . . . 45

4.1.1 Elektronischen Phasenseparation und Kolossaler Magnetowiderstand in $\mathrm{LPCMO}$. . . . . . . . . . . . . . . . . . . . . . . . . . . . 45

4.1.2 Präparation von LCMO/PCMO Überstrukturen . . . . . . . . . . . . 49

4.1.3 Elektronische und Magnetische Charakterisierung von LCMO/PCMO Überstrukturen . . . . . . . . . . . . . . . . . . . 56

4.1.4 Diskussion und Vergleich der künstlichen und intrinsischen elektronischer Phasenseparation in LCMO/PCMO und LPCMO . . . . . . . . 60

4.2 Künstliche A-Platz Ordnung in LSMO . . . . . . . . . . . . . . 67

4.2.1 Präparation von LSMO mit MAD . . . . . . . . . . . . . . 70

4.2.2 Strukturelle Eigenschaften der LSMO Übergitter . . . . . . . . . . . . 78

4.2.3 Magnetische und elektrische Eigenschaften der LSMO Übergitter . . . 84

4.2.4 Diskussion der LSMO Übergitter . . . . . . . . . . . . . . . . . . . . 87

5 B-Platz Ordnung in ferri-/ferromagnetischen Doppelperowskiten $\quad 93$

5.1 B-Platz Ordnung in LMCO Filmen . . . . . . . . . . . . . . . . . . . . . 95

5.1 Elektronische Struktur von LMCO . . . . . . . . . . . . . 95

5.1 .2 Präparation von LMCO mit MAD . . . . . . . . . . . . . . 96 
5.1.3 Strukturelle und magnetische Eigenschaften von dicken LMCO Schichten auf $\mathrm{STO}(111) \ldots \ldots \ldots \ldots 1 . \ldots \ldots 1$

5.1.4 Diskussion der B-Platz Ordnung in LMCO Filmen . . . . . . . . . . . 109

5.2 Präparation von ferrimagnetischen Doppelperowskit Filmen mit MAD . . . . 117

5.2.1 Elektronische Struktur von SFMO . . . . . . . . . . . . . 117

5.2.2 Problematik in der Präparation von ferrimagnetischen DP Filmen . . 118

5.2.3 Erprobung der Low-Oxygen-MAD (LOMAD) Technologie . . . . . . 120

5.2.4 Präparation von ferrimagnetischen SFMO Filmen mittels LOMAD . . 127

5.2.5 Präparation von ferrimagnetischen DP Filmen mittels LOMAD: Ausblick133 



\section{Einleitung}

Die Klasse der oxidischen Perowskite (im folgenden Perowskite) beherbergt Verbindungen mit diversen physikalischen Phänomenen wie z.B. Ferroelektrizität, Ferromagnetismus, spinabhängiger Transport, Ladungsordnung [1], kolossaler Magnetowiderstand (CMR) [2] und Hochtemperatur-Supraleitung [3]. Die Komplexität dieser Phänomene, die aus den starken Wechselwirkungen zwischen dem Elektronen-, Spin- und Gittersystems resultiert, weckt ein hohes wissenschaftliches Interesse an dieser Materialklasse, der stark korrelierten Elektronensysteme. Zwar existieren für diese Phänomene Erklärungsansätze, sie sind jedoch noch nicht hinreichend geklärt: Beispielsweise ist der CMR und dessen Zusammenhang mit einer elektronischen Phasenseparation (PS), die in vielen CMR Systemen auftritt, nicht vollständig verstanden [4]. Darüber hinausgehend bieten diese Materialien Anwendungsmöglichkeiten in den Bereichen der Sensor- und Datenspeicherungs-Technologie (z.B. Magnetfeldsensoren mittels des kolossalem oder Tunnel-Magnetowiderstandes [5]) oder auch in der Spintronik (z.B. Spin-injektion über halbmetallische Schottky-Dioden [6]). Speziell ferro- oder ferrimagnetische Perowskite, mit der sich diese Arbeit beschäftigt, sind für die genannten Anwendungsgebiete essenziell.

Ein großes Hindernis für die Anwendbarkeit dieser Materialien in elektrotechnischen Applikationen, ist die Größe der spezifischen Temperaturen, bei der die angesprochenen physikalischen Phänomene beobachtet werden können. Die physikalischen Eigenschaften der Perowskite zeigen eine Abhängigkeit von der Ordnung der verschiedenartigen Metallionen in mehrkomponentigen Systemen. Die Abhängigkeit ergibt sich durch den Einfluss der Metallionen auf die Elektronenkonfiguration und elastischen Verspannung innerhalb des Materials. Man spricht in diesem Zusammenhang auch von der Kontrolle der Füllung und der Bandbreite der elektronischen Bänder im Material durch die Wahl der Metallionen. In der Literatur konnte bereits gezeigt werden, dass die Phasenübergangstemperatur durch Kationen-Ordnung erhöht bzw. im Prinzip erst realisiert werden kann [7-11]. Entsprechend zeigt auch der CMR eine Abhängigkeit von der Kationen-Ordnung [8, 12].

Anknüpfend an diese Studien war die Zielsetzung dieser Arbeit, die Präparation und Charakterisierung von künstlich A-Platz geordneten schmal- und breitbandigen Manganat ${ }^{1}$ Dünnfilmen als auch von natürlich B-Platz geordneten ferro-/ferrimagnetischen doppelperowskitischen Dünnfilmen. Für die Präparation der dünnen Schichten wurde die unkonventionelle Metallorganischen Aerosol Deposition (MAD) verwendet [13]. Die MAD stellt, als eine

\footnotetext{
${ }^{1}$ Auch wenn der chemisch richtige Begriff „,Manganit“ ist, wird in dieser Arbeit der allgemein gebräuchliche Begriff „Manganat" verwendet.
} 


\section{Einleitung}

innovative, Vakuum-freie, chemische Depositionsmethode zur Präparation von qualitativ hochwertigen, oxidischen Dünnfilmen, eine konkurrenzfähige, ökonomische Alternative zu physikalischen Depositionsverfahren wie dem Laserstrahlverdampfen (PLD) oder der Molekularstrahlepitaxie (MBE) dar, welche meist in den angesprochenen Studien Anwendung fanden.

Innerhalb dieser Arbeit konnte gezeigt werden, dass diverse künstlich oder natürlich Kationengeordnete Perowskite mit der MAD Technologie präpariert werden können. Die lagenweise A-Platz Ordnung in Manganaten führt, über die Modulation der Gitterverspannung und der Elektronenbesetzung im $e_{g}$-Band der Manganionen, zu modifizierten elektronischen und magnetischen Eigenschaften. In schmalbandigen CMR Manganaten wurde die PS und somit der CMR über die Ordnung beeinflusst, während in breitbandigen CMR Manganaten ein Weg aufgezeigt werden konnte, der zu Übergangstemperaturen $T_{C}>370 \mathrm{~K}$ führen kann. In geordneten, ferromagnetischen Doppelperowskiten wurde der Einfluss und die Anwesenheit von Antiphasen-Grenzen dargelegt. Über die Einführung einer aktiven Valenz-Kontrolle, konnte die Präparation von halbmetallischen, ferrimagnetischen Doppelperowskiten mit der MAD Technologie ermöglicht werden. 
Aufbau dieser Arbeit Nach einer Einführung in die theoretischen Grundlagen zur elektronischen Struktur von Perowskiten und den verwendeten experimentellen Methoden, behandelt der erste Teil des Kapitels A-Platz Ordnung in Manganaten den Einfluss einer künstlichen Phasenseparation durch lagenweise Ordnung der La- und Pr- Ionen in $\left(L a_{0.5} \operatorname{Pr}_{0.5}\right)_{0.67} \mathrm{Ca}_{0.33} \mathrm{MnO}_{3}$, einem elektronisch phasenseparierten CMR System. Im zweiten Teil dieses Kapitels wird die Auswirkung verschiedener künstlicher Ordnungsgeometrien auf den ferromagnetischen Übergang von $\mathrm{La}_{0.67} \mathrm{Sr}_{0.33} \mathrm{MnO}_{3}$ untersucht.

Die Zielsetzung des Kapitels B-Platz Ordnung in Doppelperowskiten ist die Präparation von ferrimagnetischen halbmetallischen Doppelperowskiten. Dafür wurden im ersten Unterkapitel zunächst die strukturellen und magnetischen Eigenschaften von $\mathrm{La}_{2} \mathrm{CoMnO}_{6}$ Dünnfilmen charakterisiert. Dieses ferromagnetische Doppelperowskit eignet sich als prototypisches System, um die B-Platz Ordnung von MAD präparierten Dünnfilmen dieser Materialklasse zu verifizieren und quantifizieren. Der zweite Teil dieses Abschnittes beschäftigt sich mit der Etablierung der Valenz-Kontrolle, die für die Präparation von ferrimagnetischen Doppelperowskiten mittels MAD Technik unverzichtbar ist.

In jedem Unterkapitel erfolgt eine separate Einführung in die Problemstellung, gefolgt von einem Ergebnis- und Diskussionsteil. Eine Zusammenfassung aller Ergebnisse mit Ausblick findet im letzten Kapitel statt. 



\section{Grundlagen zur elektronischen Struktur der Perow- skite}

Perowskite werden im trivialsten Fall von der chemischen Summenformel $A B O_{3}$ beschrieben, wobei der A-Platz meist von einem Erdalkali oder Seltene-Erd-Metallion und der B-Platz von einem Übergangsmetallion besetzt ist. In der idealen kubischen Darstellung sitzen die APlatz Ionen auf den Ecken, die Sauerstoffionen zentriert auf den Flächen des Kubus und die B-Platz Ionen raumzentriert innerhalb des Kubus. Die Sauerstoffionen bilden ein Oktaeder in dem raumzentriert das B-Ion sitzt (siehe Abb.1).

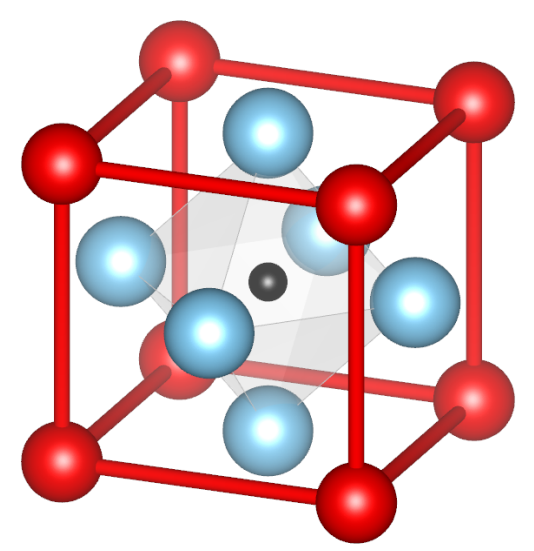

Abbildung 1: Idealisierte Darstellung eines kubischen Perowskites (hier $\mathrm{SrTiO}_{3}$ ): Das Übergangsmetallion auf dem B-Platz (Ti, schwarz) ist von einem Sauerstoff-Oktaeder (blau) umgeben. Der Oktaeder ist von einem Kubus umgeben auf dessen Ecken sich die A-Platz Ionen (Sr, rot) befinden. Die roten Verbindungslinien sollen dabei keine Atombindungen darstellen.

Der Ursprung der beschriebenen physikalischen Phänomene lässt sich in den $d$ Orbitalen des Übergangsmetallions auf dem B-Platz finden. Zwischen den B-Plätzen wechselwirken die $d$ Elektronen über die $p$ Orbitale des Sauerstoffes miteinander (siehe Kapitel 2.2). Die Anzahl der $d$-Elektronen wird durch die Wahl der Ionen auf dem A- und B-Platz definiert. Zusätzlich bewirkt das Volumen der A- und B-Platz Ionen Verspannungen in der Kristallstruktur, die wiederum die Physik der $d$-Elektronen beeinflusst. Somit hat eine Ordnung der A- und/oder B-Platz Ionen durch den Einfluss auf die $d$-Elektronenverteilung und Verspannung im Gitter, auch Einfluss auf die physikalischen Eigenschaften des oxidischen Perowskites. In dieser Arbeit wird die Wirkung künstlicher und selbst getriebener Kationen-Ordnung in speziellen ferromagnetischen, halbmetallischen Manganaten (Summenformel: $A_{x} A_{1-x}^{\prime} M n O_{3}$ ) und ferri- oder ferromagnetischen Doppelperowskiten (Summenformel: $A_{2} B B^{\prime} O_{6}$ ) auf die elektronischen und magnetischen Eigenschaften untersucht. Nach einer theoretischen Einführung, 
über den Einfluss der Kristallstruktur auf die Besetzung der $d$ Orbitale, werden die möglichen magnetischen Austauschmechanismen in diesen Systemen kurz beschrieben.

\subsection{Einfluss der Struktur auf die orbitale Besetzung}

\subsubsection{Die Kristallfeldaufspaltung}

In einem isolierten Übergangsmetallion sind die äußeren fünf $d$ Orbitale $\left(d_{x y}, d_{x z}, d_{y z}, d_{z^{2}}\right.$, $d_{x^{2}-y^{2}}$ ) auf einem energetischen Niveau (man spricht auch von einer 10-fachen Entartung). Sitzt dieses Ion jedoch auf dem B-Platz eines oxidischen Perowskites, ist es von einem Sauerstoffoktaeder umgeben. Betrachtet man die geometrische Form des Raumes der höchsten Elektronen-Aufenthaltswahrscheinlichkeit für die verschiedenen $d$ Orbitale, so stellt sich heraus, dass Elektronen im $d_{z^{2}}$ und $d_{x^{2}-y^{2}}$ Orbital $\left(e_{g}\right.$ Orbitale) sich näher an den elektrisch negativ geladenen $p$-Orbitalen der Sauerstoffionen befinden als Elektronen im $d_{x y}, d_{x z}$ oder $d_{y z}$ Orbital ( $t_{2 g}$ Orbitale). Aufgrund der Coulomb-Wechselwirkung kommt es zu einer energetischen Aufspaltung der d-Orbitale in $e_{g}$ und in energetisch günstigere $t_{2 g}$ Orbitale (siehe Abb.2).

Der Energieunterschied zwischen $e_{g}$ und $t_{2 g}$ wird Kristallfeldenergie $E_{C F}$ genannt. Diese Energie kann einen Einfluss auf die Verteilung der Elektronen innerhalb des d-Orbitals haben. Ist $E_{C F}$ kleiner als die Hund'sche Kopplungsenergie $E_{H}>E_{C F}$ (die Energie, die benötigt wird, um ein Orbital mit zwei Elektronen mit antiparallelen Spins zu besetzen) spricht man von der high-spin Konfiguration: die $e_{g}$ Orbitale werden mit Elektronen besetzt, bevor ein $t_{2 g}$ Orbital mit einem zweiten Elektron besetzt wird. Ist $E_{H}<E_{C F}$ so kommt es in $d$ Orbitalen, die mit 4 - 7 Elektronen besetzt sind, zur low-spin Konfiguration bei der zunächst alle $t_{2 g}$ Orbitale mit jeweils zwei Elektronen besetzt werden, bevor das erste $e_{g}$ Orbital besetzt wird [14]. Für Manganate liegt die Kristallfeldenergie bei $E_{C F} \sim 1 \mathrm{eV}$ während $E_{H} \sim 2-3 \mathrm{eV}$ beträgt [15]. Somit liegt in diesem Material für das $M n$ eine high-spin Konfiguration vor.

\subsubsection{Der Jahn-Teller Effekt}

In $d^{9}$, low-spin $d^{7}$ und high-spin $d^{4}$ Perowskiten kann es neben der Kristallfeldaufspaltung zu einer zusätzlichen Jahn-Teller-Aufspaltung zwischen den Energien der $d$ Orbitale kommen. Ein $e_{g}$ Orbital ist in diesen Konfigurationen halb besetzt, z.B. ist in der high-spin $d^{4}$ Konfiguration das $d_{z^{2}}$ oder $d_{x^{2}-y^{2}}$ Orbital mit einem Elektron besetzt. Aufgrund der Coulombabstoßung zwischen dem Elektron im $e_{g}$ Orbital und den Elektronen im $p$ Orbital des 


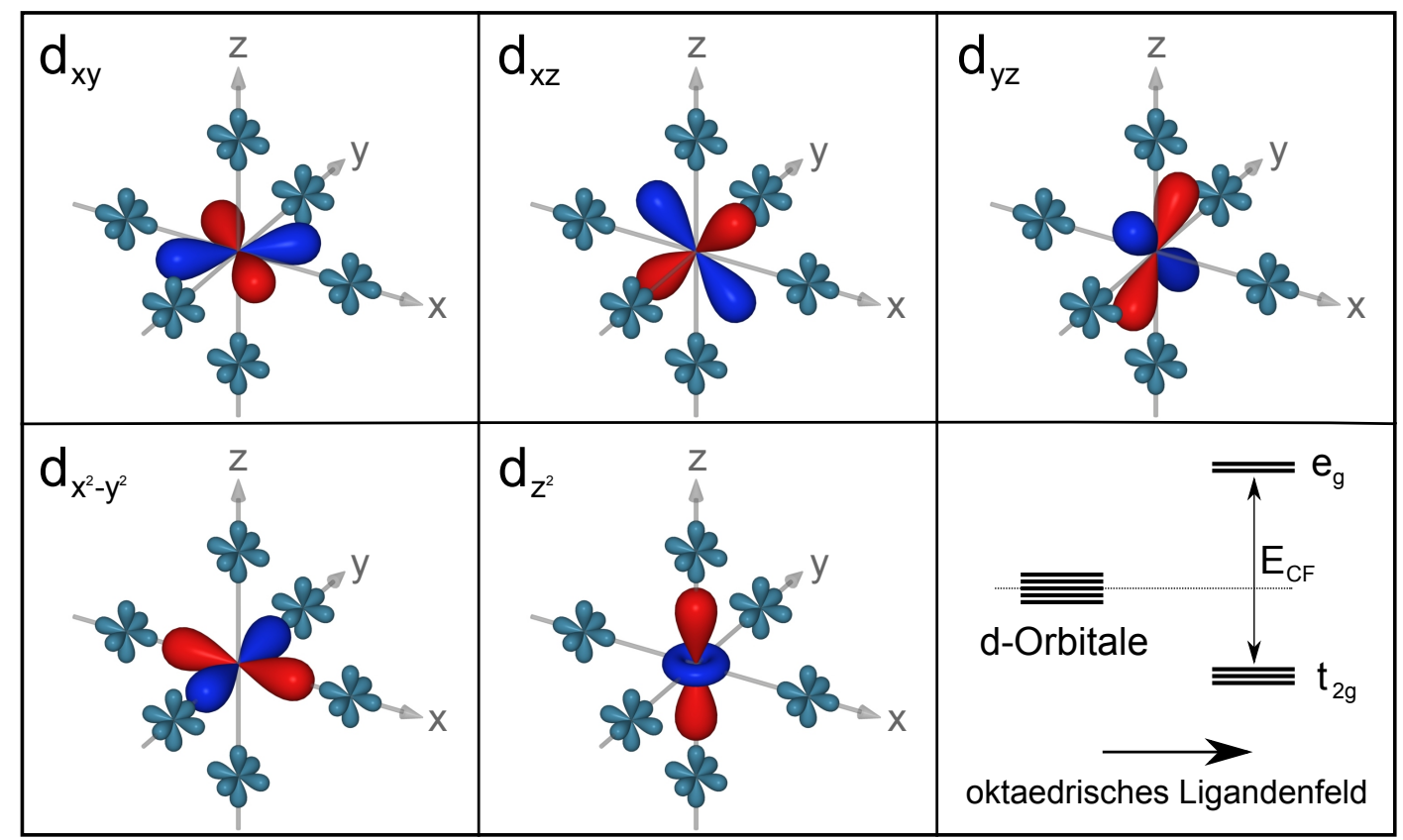

Abbildung 2: Oktaedrische Kristallfeldaufspaltung: Die beiden $e_{g}$ Orbitale $d_{z^{2}}$ und $d_{x^{2}-y^{2}}$ zeigen auf die voll besetzten $p$ Orbitale der Sauerstoffionen (als türkise Struktur verkleinert dargestellt) und liegen deshalb energetisch höher, als die $t_{2 g}$ Orbitale $d_{x y}, d_{x z}$ und $d_{y z}$, die zwischen den $O^{2-}$ Ionen hindurch zeigen. Dadurch ergibt sich die Kristallfeldaufspaltung (unten rechts). Vorzeichen der d Orbital Wellenfunktionen: $+=$ rot, -=blau.

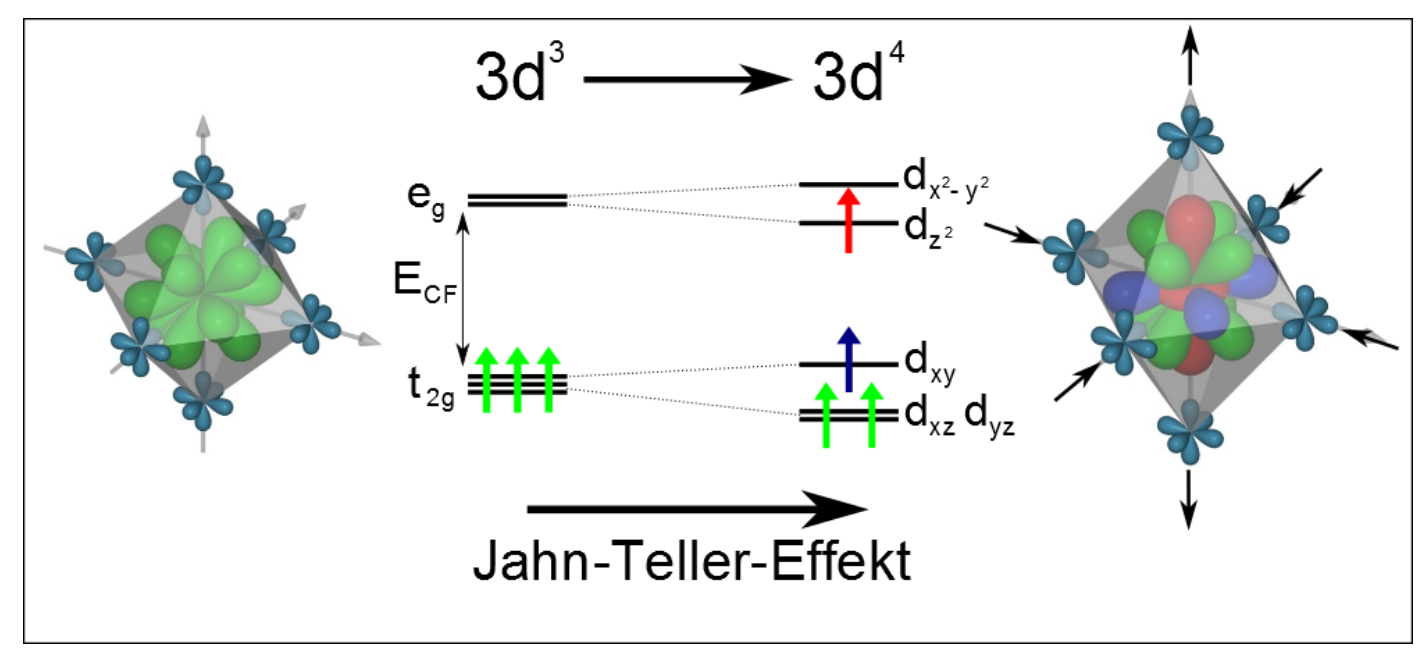

Abbildung 3: Auf der linken Seite ist ein $3 d^{3}$ Perowskit dargestellt. Jedes energetisch gleichwertige $t_{2 g}$ Orbital ist dabei mit einem Elektron besetzt. Fügt man ein weiteres Elektron zu diesem high-spin Zustand hinzu, findet sich dieses in einem $e_{g}$ Orbital wieder. Ist es im $d_{z^{2}}$ Orbital zu finden, wie auf der rechten Seite dargestellt, wird der Sauerstoff-Oktaeder, wie im Text beschrieben, deformiert. Es kommt zu einer weiteren Aufspaltung der Orbitale (Jahn-Teller-Effekt). Die Elektronen sind entsprechend ihrem d Orbital energieabhängig eingefärbt. 
Sauerstoffes wird der Sauerstoffoktaeder um das zentrale Übergangsmetallion verzerrt. Sitzt dabei das Elektron im $d_{z^{2}}$ Orbital, kommt es zu einer Streckung des Oktaeders (wie in Abb.3 dargestellt). Sitzt das Elektron im $d_{x^{2}-y^{2}}$ Orbital, kommt es zu einer Stauchung des Oktaeders. Diese Verzerrung führt wiederum zu einer energetischen Aufspaltung der $e_{g}$ als auch der $t_{2 g}$ Orbitale. Im ersten Fall, indem der Oktaeder gestreckt wird, wird das $d_{z^{2}}, d_{x z}$ und $d_{y z}$ Orbital aufgrund des größeren Abstandes zum Sauerstoff energetisch abgesenkt, während das $d_{x^{2}-y^{2}}$ und das $d_{x y}$ Orbital energetisch erhöht wird [14]. Diese Energieaufspaltung hat in den pseudokubischen Manganaten eine Größenordnung von $\sim 0.25 \mathrm{eV}$ [15].

\subsubsection{Einfluss der Fehlanpassung zwischen Substrat und Dünnfilmen}

Die Fehlanpassung, aufgrund des Unterschiedes der Gitterkonstanten von Substrat und Dünnfilm, resultiert in einer Verspannung des epitaktisch gewachsenen dünnen Filmes. Sind die Gitterparameter des dünnen Filmes, senkrecht zu dessen Wachstumsrichtung, größer (kleiner) als die entsprechenden Gitterparameter des Substrates, erfährt die Struktur des Filmes eine Druckspannung (Zugspannung). Die Änderung der Seitenverhältnisse durch den Jahn-Teller-Effekt in $d^{9}$, low-spin $d^{7}$ und high-spin $d^{4}$ Perowskiten, führt zu einer bevorzugten Besetzung der $e_{g}$ Orbitale. Während unter der Zugspannung das $d_{x^{2}-y^{2}}$ Orbital energetisch günstiger ist, wird unter Druckspannung das $d_{z^{2}}$ Orbital bevorzugt $[16,17]$. In der Literatur wird weiter diskutiert, dass neben der Verspannung durch die Fehlanpassung, auch die Symmetriebrechung an den Grenzflächen eines dünnen Filmes zu einer orbitalen Rekonstruktion führen kann. Im Falle des $\mathrm{La}_{0.7} \mathrm{Sr}_{0.3} \mathrm{MnO}_{3}$ wurde eine verstärkte Besetzung des $d_{z^{2}}$ Orbitals registriert [18].

\subsection{Austauschmechanismen in Perowskiten}

Die Austauschwechselwirkung ist ein rein quantenmechanischer Effekt. Dieser Effekt ermöglicht erst eine langreichweitige magnetische Ordnung, die zu Ferro- oder Antiferromagnetismus (FM oder AFM) führt, bei hohen Temperaturen (von mehreren hundert Kelvin). Dabei existiert eine maximale Temperatur bis zu der, die Ordnung beobachtet werden kann. Im Falle des FMs wird sie als Curie Temperatur $T_{C}$ und im Falle des AFMs als Néel Temperatur $T_{N}$ bezeichnet.

Die Austauschwechselwirkung basiert auf dem Prinzip, dass der Austausch (virtuell oder real) zweier quantenmechanisch ununterscheidbaren Teilchen wieder zum gleichen Zustand führt. Somit muss für eine vollständige Beschreibung eines quantenmechanischen Systems 
mit zwei oder mehr Elektronen beachtet werden, dass die Vertauschung der Teilchen die Wellenfunktion, jedoch nicht das Betragsquadrat der Wellenfunktion, des Systems ändert. Die Änderung der Wellenfunktion durch Elektronenaustausch resultiert aus der antisymmetrischen Vertauschung bei Fermionen (Spin-Statistik-Theorem) und führt dazu, dass jeder Zustand (Energie und Spin) nur genau von einem Elektron besetzt werden kann. Die Identität des Betragsquadrates bedeutet, dass in beiden Zuständen die gleiche Elektronendichte und damit der gleicher makroskopischer Zustand vorliegt. Folglich sind die Elektronen und somit auch deren Spins verschränkt und die vertauschten quantenmechanischen Zustände müssen berücksichtigt werden. Für den Erwartungswert des Hamiltonoperators ergibt sich somit ein zweiter Summand neben der direkten Wechselwirkung, die sogenannte Austauschenergie. Diese kann zu einer Zunahme oder Abnahme der Wechselwirkungsenergie führen. In einem Festkörper mit halb-besetzten Elektronenzuständen (Elektronen an der Fermikante) kann die Austauschenergie, unter der Berücksichtigung der Coulombabstoßung zwischen den Elektronen, zu einer Ordnung der Elektronenspins führen. Diese erzeugt im ferromagnetischen Fall (Spins parallel ausgerichtet) eine remanente Magnetisierung. Weitere Ausführungen zu diesem Thema findet man in weiterführender Literatur [19].

In Übergangsmetalloxiden existiert nur eine geringe direkte Überlappung zwischen den teilgefüllten $d$ Orbitalen, was zu einer verschwindenden direkten Austauschwechselwirkung führt. In den beiden folgenden Unterkapiteln werden zwei Mechanismen für eine Austauschwechselwirkung in Übergangsmetalloxiden über die $p$ Orbitale des Sauerstoffes vorgestellt, die zu einer magnetischen Ordnung in diesen Materialien führen kann.

\subsubsection{Der Superaustausch}

Der Superaustausch (SE) beschreibt eine Austauschwechselwirkung zwischen lokalisierten $d$-Elektronen der Übergangsmetallionen über die $p$-Elektronen des Sauerstoffes [20] [21].

Aufgrund der Heisenbergschen Unschärferelation ist die Energie eines delokalisierten Teilchens kleiner als die eines lokalisierten Teilchens. Bezogen auf die Elektronen in einem Übergangsmetalloxid bedeutet das, dass die Energie eines lokalisierten Elektrons, welches die Möglichkeit eines Transfers in ein Orbital des Nachbarions hat und damit stärker delokalisiert ist, vermindert wird. Die sogenannte Hüpfamplitude $t$ ist ein Maß für die im Transfer gewonnene kinetische Energie. Es ist noch zu bedenken, dass eine potentielle Energie $U$ aufgewendet werden muss, um ein zusätzliches Elektron in das Nachbar-Orbital zu setzen. Bei lokalisierten Elektronen ist $t<<U$. Mithilfe der Störungstheorie zweiter Ordnung gewinnt man mit $t^{2} / U$ ein Maß für den Energiegewinn aus der Möglichkeit des virtuellen Transfers 
2 Grundlagen zur elektronischen Struktur der Perowskite

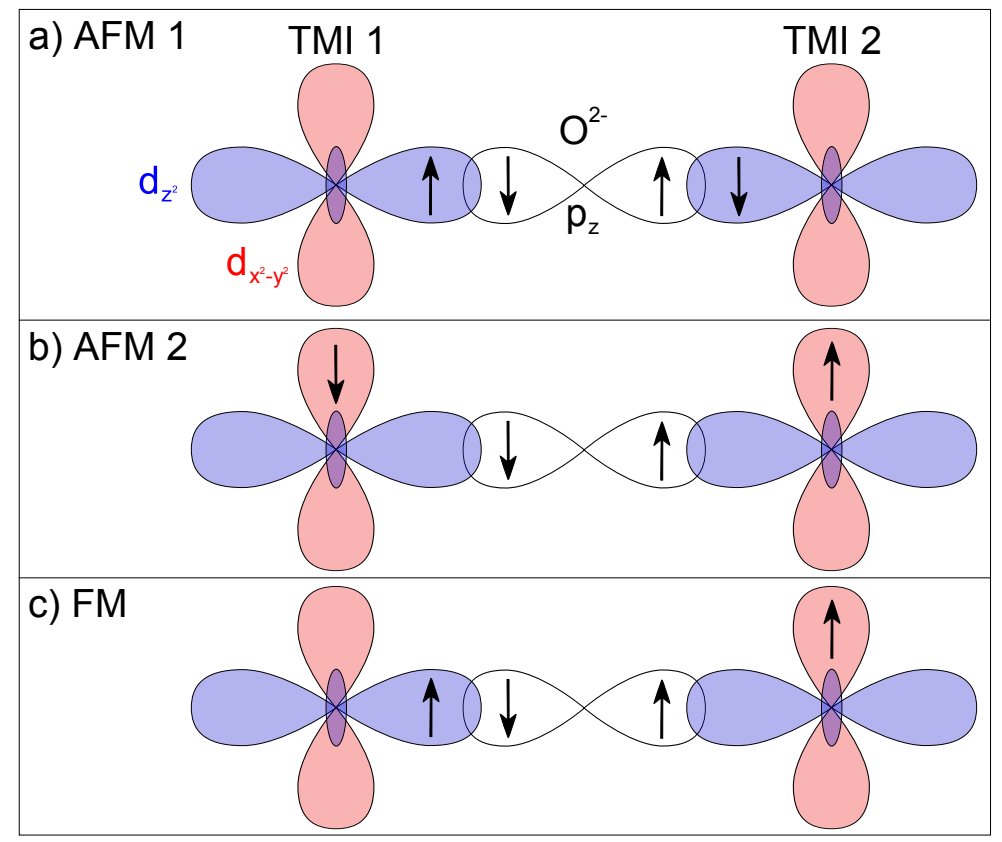

Abbildung 4: SE anhand eines vereinfachtes $e_{g}^{1}-O-e_{g}^{1}$ Systems. In a) befinden sich beide $e_{g}$ Elektron im $d_{z^{2}}$ Orbital. Die Gesamtenergie ist minimal durch das Einstellen der dargestellten AFM-Ordnung. Gleiches gilt für den Fall in b) in dem beide $d_{z^{2}}$ Orbitale leer sind. Für die Konfiguration in c) stellt sich eine FM Ordnung ein. Eine genaue Ausführung befindet sich im Text.

für ein lokalisiertes Elektron.

Geht man von einem direkten $d$ Orbital zu $d$ Orbital Wechselwirkung aus, muss betrachtet werden, ob bereits ein Elektron im Nachbar-Orbital vorhanden ist. Wenn ein Elektron im Nachbar-Orbital vorhanden ist, können beide betrachteten Elektronen den selben oder einen unterschiedlichen Spin haben. Im ersten Fall muss zusätzlich die Hund'sche Kopplungsenergie aufgebracht werden, damit der Wechselwirkung durch das Pauli-Prinzip nicht verboten ist. Die Wechselwirkung findet außerdem nicht statt, wenn das Nachbar-Orbital mit zwei Elektronen voll besetzt ist.

Jedoch existiert in Übergangsmetalloxiden nahezu keine direkte $d$ zu $d$ Wechselwirkung aufgrund der geringen Überlappung der Orbitale. Stattdessen findet die Wechselwirkung über die vollbesetzten $2 p$ Orbitale des Sauerstoffions zwischen zwei Übergangsmetallionen statt. Im einfachsten Fall können die zwei Elektronen im $p$ Orbital des Sauerstoffes als Vermittler zwischen den $d$ Elektronen der Übergangsmetallionen angesehen werden. Je nach der orbitalen Konfiguration der Übergangsmetallionen kann sich ein AFM- oder FM-Austausch zwischen den Spins ihrer $d$-Elektronen ergeben. Zur Veranschaulichung betrachten wir ein vereinfachtes $e_{g}^{1}$-O- $e_{g}^{1}$ System, wie in Abb.4 dargestellt. In Abb.4 a) befindet sich das $e_{g}$-Elektron in beiden Übergangsmetallionen im $d_{z^{2}}$ Orbital. Bei einer AFM-Ordnung der Spins beider 
$e_{g}$-Elektronen, können beide $p$-Elektronen im $p_{z^{-}}$Orbital des Sauerstoffes in die $d_{z^{2}}$-Orbitale der benachbarten Übergangsmetallionen delokalisieren. Somit wird die Energie des Systems minimiert. Haben die Spins der $e_{g}$-Elektronen jedoch eine FM-Ordnung, muss zusätzlich die Hund'sche Kopplungsenergie aufgebracht werden, damit beide $p$-Elektronen delokalisieren können. Aufgrund der höheren Minimierung der Gesamtenergie stellt sich in diesem Fall eine AFM-Ordnung ein. Gleiches gilt auch für den in Abb.4 b) dargestellten Fall, bei dem sich die $e_{g}$-Elektronen in beiden Übergangsmetallionen im $d_{x^{2}-y^{2}}$-Orbital befinden. Befindet sich ein $e_{g}$-Elektron eines Übergangsmetallions im $d_{z^{2}}$-Orbital und das $e_{g}$-Elektron des zweiten Übergangmetallions im $d_{x^{2}-y^{2}}$-Orbital, wie in Abb.4 c) dargestellt, muss der Spin des $p_{z}$-Elektrons, das dem leeren $d_{z^{2}}$-Orbital gegenübersteht, unter Berücksichtigung des PauliPrinzips, parallel zum Spin des $d_{x^{2}-y^{2}}$-Elektrons sein, damit es delokalisieren kann. Das zweite $p_{z}$ Elektron muss dagegen, wie in den letzten beiden Fällen, eine antiparallelen Spin im Vergleich zu dem benachbarten $d_{z^{2}}$ Elektron vorweisen. Hieraus ergibt sich eine FM Ordnung. Mit dem $e_{g}$-Spin ist ein möglicherweise vorhandener $t_{2 g}$-Spin über das Pauli-Prinzip gekoppelt.

Aus diesen Betrachtungen folgen zusammenfassend die sogenannten Goodenough-KanamoriAnderson Regeln (GKA-Regeln) für den SE, welche die magnetische Austauschwechselwirkung in den meisten Fällen richtig vorhersagt [22] [23]:

1. Der $180^{\circ}$ Austausch zwischen zwei leeren oder halb-gefüllten Orbitalen ist stark und antiferromagnetisch (Abb.4 a und b).

2. Der $180^{\circ}$ Austausch zwischen einem halb-gefüllten und einem leeren Orbital ist schwach und ferromagnetisch (Abb.4 c).

3. Der $90^{\circ}$ Austausch zwischen zwei halb-gefüllten Orbitalen ist schwach und ferromagnetisch.

An diesem Punkt sei noch bemerkt, dass, aufgrund der Struktur, nur die ersten zwei GKA Regeln in Perowskiten Anwendung finden. Der 90 Austausch kann in Übergangsmetalloxiden mit Natriumchlorid-Struktur wie $\mathrm{CoO}$ Anwendung finden und wird in dieser Arbeit nicht näher erläutert.

Die Größe der Hüpfamplitude $t$ und damit die Größe der Energieminimierung durch den SE hängt nach Definition direkt von der Überlappung zwischen den zwei am Hüpf-Prozess beteiligten Orbitalen ab. Die $e_{g}$ Orbitale haben in Perowskiten eine direkte Überlappung mit den $p$ Orbitalen des Sauerstoffes, sie gehen miteinander eine kovalente $\sigma$-Bindung ein. Auch 
die Elektronen der $t_{2 g}$ Orbitale können mit den $p$-Elektronen des Sauerstoffs über eine $\pi$ Bindung zwischen den Orbitalen wechselwirken. Die Energien im $\pi$-SE sind jedoch, aufgrund der kleineren Überlappung der Orbitale, geringer als die im $\sigma$-SE.

\subsubsection{Der Doppelaustausch}

Der Doppelaustausch (DE) wurde zunächst von C. Zener [24] vorgeschlagen und später von P.W. Anderson, H. Hasegawa [25] und P.-G. de Gennes [26] quantitativ weiterentwickelt. Ziel war es den von G.H. Jonker und J.H. van Santhen experimentell beobachteten Ferromagnetismus [27] und die Änderung der Leitfähigkeit in der nähe der Curie-Temperatur [28] in $\mathrm{La}_{1-x} A_{x} \mathrm{MnO}_{3}$ mit $\mathrm{A}=\mathrm{Ca}, \mathrm{Sr}, \mathrm{Ba}$, zu erklären. Wie auch schon der SE, beschreibt das Modell des DE eine Austauschwechselwirkung zwischen den $d$-Orbitalen der Übergangsmetallionen über das p-Orbital des Sauerstoffes. Im Unterschied zu dem SE, kann der DE den Magnetismus in Übergangsmetalloxiden erklären, in denen die $e_{g}$ Elektronen reell delokalisiert sind, d.h. in Übergangsmetalloxiden, die ein metallisches Verhalten zeigen. Im, für den DE prototypischen, $\mathrm{La}_{1-x}^{3+} A_{x}^{2+} \mathrm{MnO}_{3}$ System sind, aufgrund des gemischtvalent besetzten A-Platzes, sowohl $M n^{3+}$ als auch $M n^{4+}$-Ionen vorhanden. Die $d$-Orbitale der Mangan-Ionen sind durch das oktaedrische Kristallfeld dieses Perowskites aufgespalten: Die drei $d$-Elektronen des $M n^{4+}$ befinden sich in den $t_{2 g}$ Orbitalen mit paralleler Spin-Ausrichtung (Hund'sches Gesetz). Das $M n^{3+}$ liegt, wie man aus der Tieftemperatur Sättigungsmagnetisierung schließen kann, in diesem Material in der high-spin Konfiguration vor [27]. Folglich befindet sich das vierte $d$-Elektron des $M n^{3+}$ in einem $e_{g}$-Orbital, mit einen zu den $t_{2 g}$-Elektronen parallelen Spin. In Abb. 5 sind diesen beiden Mn-Ionen mit unterschiedlicher Valenz über den Sauerstoff miteinander verknüpft. Wie bereits im Abschnitt über den SE erwähnt, kann die Gesamtenergie des Systems durch die Delokalisierung von Elektronen erniedrigt werden. Die Gesamtenergie kann folglich minimiert werden, wenn das $e_{g}$-Elektron des $M n^{3+}$ reell in das leere $e_{g}$-Orbital des $M n^{4+}$ delokalisiert. Ein direkter Transfer des Elektrons zwischen den $M n$-Ionen ist aufgrund der geringen Überlappung der $d$-Orbitale sehr unwahrscheinlich. Der Vorschlag von Zener [24] war, dass gleichzeitig das $e_{g^{-}}$ Elektron des $\mathrm{Mn}^{3+}$ in das $p$-Orbital des Sauerstoffs und das $p$-Elektron des Sauerstoffs mit dem gleichen Spinzustand wie das $e_{g}$-Elektron in das leere $e_{g}$-Orbital des $M n^{4+}$ transferiert wird (siehe Abb.5).

Sind die magnetischen Momente der beteiligten $M n$-Ionen antiparallel ausgerichtet (AFM Fall in Abb.5 b), muss bei dem beschriebenen Elektronentransfer zusätzlich die Hund'sche Kopplungsenergie für das transferierte $e_{g}$-Elektron aufgebracht werden. Typischerweise über- 


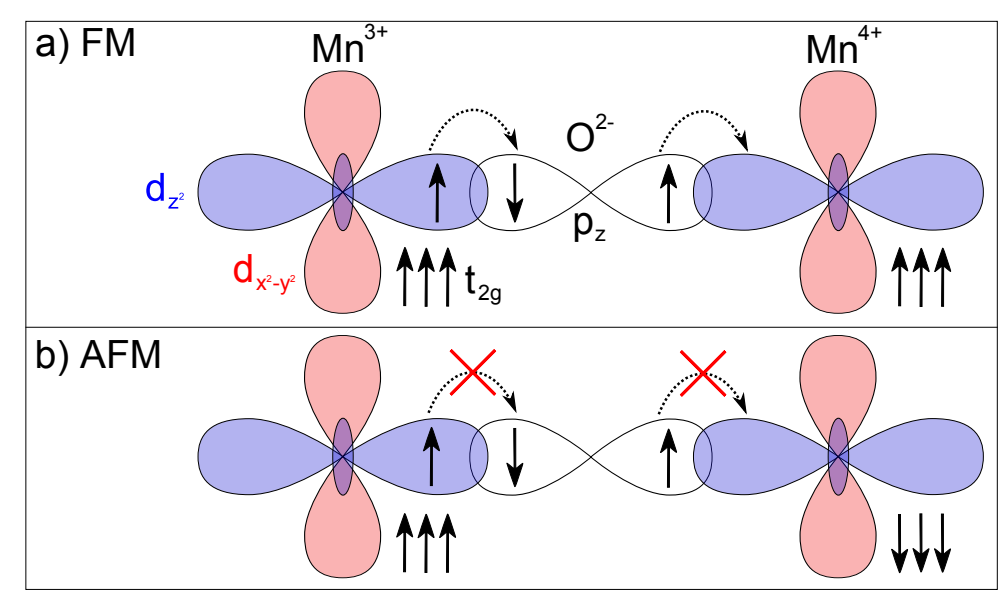

Abbildung 5: Schematische Darstellung des DE. Haben die magnetischen Momente der Mn-Ionen eine FM Ordnung (a), kann der DE über den Sauerstoff stattfinden. Dabei werden gleichzeitig die Elektronen mit gleichen Spin aus dem $\mathrm{Mn}^{3+} e_{g}$-Orbital und dem $\mathrm{O}^{2-}{ }^{-}$-Orbital, wie in der $\mathrm{Ab}$ bildung durch die gepunkteten Pfeile angedeutet, transferiert. Bei einer AFM Ordnung der magnetischen Momente der Mn-Ionen (b), findet der DE nicht statt, da in diesem Fall zusätzlich die Hund'sche Kopplungsenergie aufgebracht werden müsste. Eine genaue Ausführung befindet sich im Text.

wiegt die Hund'sche Kopplungsenergie der Minimierung der Gesamtenergie durch die Delokalisierung der $e_{g}$-Elektronen. Somit findet der $e_{g}$-Elektronentransfer nicht statt. Für eine parallele Ausrichtung der $M n$ - 3d-Elektronen (FM Fall in Abb.5 a) muss die Hund'sche Kopplungsenergie nicht aufgebracht werden und der $e_{g}$-Elektronentransfer kann stattfinden. Folglich kann die effektive Hüpfamplitude $t_{e f f}$ und damit die Leitfähigkeit der $e_{g}$-Elektronen durch

$$
t_{\text {eff }}=t \cdot \cos \frac{\theta_{i j}}{2}
$$

angeben werden, wobei $\theta_{i j}$ der Winkel zwischen den $3 d$-Elektronen-Spins der $M n$-Ionen und $t$ ein Maß für die Breite des Bandes der $e_{g}$-Elektronen ist. Zusätzlich ist die effektive Hüpfamplitude über $t$ auch direkt von den orbitalen Überlappung der Sauerstoff $p$-Orbitale und der Mangan 3d-Orbitale abhängig. Zusammenfassend ist im Modell des DE eine ferromagnetische Ordnung der magnetischen Momente der $M n$ - 3d-Elektronen energetisch bevorzugt. Diese Ordnung ist direkt mit dem Auftreten einer hohen Leitfähigkeit der $e_{g}$-Elektronen gekoppelt. Oberhalb der Curie-Temperatur $T_{C}$ stellt sich ein paramagnetischer Zustand in dem DE-Material ein, d.h. die magnetischen Momente der $M n$-Ionen sind zufällig ausgerichtet. Nach Gleichung (1) erniedrigt sich $t_{\text {eff }}$ im Vergleich zu der ferromagnetischen Ordnung mit $\theta_{i j}=0^{\circ}$ bei Temperaturen $T<T_{C}$. Damit lässt sich die Änderung der Leitfähigkeit bei $T_{C}$ in $\mathrm{La}_{1-x} \mathrm{~A}_{x} \mathrm{MnO}_{3}$ erklären. 


\subsection{Einfluss der Gitterverzerrungen auf die Austauschwechselwir- kungen}

Wie in den letzten zwei Unterabschnitten beschrieben, hängen die magnetischen und elektrischen Eigenschaften in oxidischen Perowskiten stark von der Überlappung, zwischen den $p$-Orbitalen der Sauerstoff-Ionen und den $d$-Orbitalen der Übergangsmetall-Ionen sowie der Besetzung der Orbitale, bzw. der orbitalen Ordnung, ab. Diese beiden Faktoren hängen wiederum stark von der Struktur des Materials ab. Beispielsweise kann eine Druckspannung (Zugspannung) in dem prototypischen DE-System $\mathrm{La}_{0.7} \mathrm{Sr}_{0.3} \mathrm{MnO}_{3}$, wie in Kap. 2.1.3 bereits angesprochen, zu einer präferierten Besetzung der $d_{z^{2}}\left(d_{x^{2}-y^{2}}\right)$ Orbitale (Jahn-TellerEffekt) und somit zu einer orbitalen Ordnung führen. Diese orbitale Ordnung ruft über den SE eine AFM Wechselwirkung hervor [16-18, 29]. Die FM DE Austauschwechselwirkung in $\mathrm{La}_{0.7} \mathrm{Sr}_{0.3} \mathrm{MnO}_{3}$ wird somit unter Verspannung abgeschwächt, bzw. von einer AFM SE Austauschwechselwirkung abgelöst [17]. Zusätzlich führt die Verspannung der Struktur eines dünnen Filmes durch die Fehlanpassung zum Substrat auch zu einer Änderung der angesprochene orbitalen Überlappung. Diese wird zudem von den Verspannungen beeinflusst, die durch die Fehlanpassung der Größe der vorhandenen Ionen innerhalb eins Materials verursacht werden. Die verschiedenen Größen der Ionen einer Komposition führen zu einer geometrische Fehlanpassung in der Perowskitstruktur. Diese Fehlanpassung schlägt sich in einer Änderung der Überlappung der erwähnten Orbitale nieder. Der Goldschmidt Toleranzfaktor (TF) ist ein Maß für die geometrische Fehlanpassung der A- und B-Ionen in der Perowskitstruktur. Im idealen kubischen Perowskit hätte das Verhältnis, zwischen dem Abstand von A-Ion und Sauerstoff $d_{A-O}$ und von B-Ion und Sauerstoff $d_{B-O}$, einen Wert von $\sqrt{2}$. Der TF wird aus diesem Grund folgendermaßen definiert [30]:

$$
T F=\frac{d_{A-O}}{\sqrt{2} d_{B-O}}
$$

Näherungsweise können die Metallionen-Sauerstoff-Abstände durch die entsprechenden Ionenradien ersetzt werden:

$$
T F=\frac{r_{A}+r_{O}}{\sqrt{2}\left(r_{B}+r_{O}\right)}
$$

Für mehr-komponentige Systeme lässt sich der TF über den Mittelwert der entsprechenden Ionenradien $\left\langle r_{A}\right\rangle$ bzw. $\left\langle r_{B}\right\rangle$ ermitteln. Das ideale kubische, oxidische Perowskit ist $\mathrm{SrTiO}_{3}$ (STO) mit einem TF von 1.0. Für TF zwischen 1.0 und 0.98 wird meist die kubische, im unteren Bereich des Intervalls jedoch auch die rhomboedrische Kristallstruktur angetroffen. Zwischen 0.98 und 0.96 stellt sich eine orthorombische Struktur ein. Für einen TF kleiner 
als 0.96 wird die Ilmenit-Struktur und für TF größer als 1.00 die hexagonale Kristallstruktur energetisch günstiger [15]. Die Änderung des TFs geht mit einer kontinuierlichen Änderung des Bindungswinkels $\angle(B-O-B)$ zwischen den B-Platz Ionen und Sauerstoffionen einher (Verkippung der Sauerstoffoktaeder). Der Winkel beträgt $180^{\circ}$, wenn $T F=1.0$ ist. Aufgrund der Geometrie der $p$-Orbitale, führt ein Winkel $\neq 180^{\circ}$ zu einer kleineren Überlappung der Orbitale. Aus diesem Grund ist der Toleranzfaktor auch ein Maß für die Überlappung, der an den Austauschmechanismen beteiligten Orbitale.

In mehr-komponentigen Perowskiten, speziell in Manganaten, hat neben dem Mittelwert der Ionenradien, der in den TF eingeht, auch die Varianz der Ionenradien einen großen Einfluss auf den Austauschmechanismus. Sie beschreibt die Unordnung in dem System, welche durch das Vorhandensein von verschieden großen Ionen hervorgerufen wird. In Systemen mit nur einer Art von B-Platz Ionen (wie die Manganate) und mit $i$ unterschiedlichen A-Ionen ist die Varianz über $\sigma^{2}=\sum_{i} y_{i} r_{i}^{2}-\left\langle r_{A}\right\rangle^{2}$ definiert. Variable $y_{i}$ ist dabei der relative Anteil des A-Platz Ions $i$ mit dem Radius $r_{i}$ und $\left\langle r_{A}\right\rangle$ der Mittelwert der Radien aller A-Platz Ionen. Zusammen mit dem Einfluss des Toleranzfaktors kann für optimal dotierte Manganate mit $\operatorname{Re}_{0.7}^{3+} A_{0.3}^{2+} M n_{0.7}^{3+} M n_{0.3}^{4+} O_{3}$ die Curie Temperatur empirisch abgeschätzt werden durch [7]:

$$
T_{C}\left(\left\langle r_{A}\right\rangle, \sigma^{2}\right)=T_{C, \max }-p_{1} \cdot \sigma^{2}-p_{2} \cdot\left(r_{A}(T F=1)-\left\langle r_{A}\right\rangle\right)^{2}
$$

Der Zusammenhang zwischen $T_{C}$ und $\sigma^{2}$ über den empirische Faktor $p_{1}=2.06 \cdot 10^{7} \mathrm{~K} / \mathrm{nm}^{2}$ wurde mittels einer Bulk-Proben Serie mit $\sigma^{2}=1.6 \ldots 24 \cdot 10^{-5} \mathrm{~nm}^{2}$ und $\left\langle r_{A}\right\rangle=$ const $=$ $0.123 \mathrm{~nm}$ ermittelt. Dabei wurde $p_{2}=2.9 \cdot 10^{7} \mathrm{~K} / \mathrm{nm}^{2}$ über eine Variation von $\left\langle r_{A}\right\rangle$ bestimmt, wobei die hier verwendeten Werte auf $\sigma^{2}=0$ korrigiert wurden [7]. Der Radius $r_{A}(T F=1)$ ist der mittlere Radius der A-Platz Ionen, der benötigt wird damit Toleranzfaktor $T F=1$ ist. Für die hier angegebene Manganat-Dotierung ist $r_{A}(T F=1)=0.13 \mathrm{~nm}$. Aus den empirischen Daten lässt sich mit $T_{C, \max }=530 \mathrm{~K}$, die maximale Curie Temperatur extrapolieren, die in Manganaten für $\sigma^{2}=0$ und $T F=1$ theoretisch erreicht werden kann. Die bisher höchste erreichte Übergangstemperatur in dieser Art von Manganaten kann mit $T_{C}=370 \mathrm{~K}$ in $\mathrm{La}_{0.7} \mathrm{Sr}_{0.3} \mathrm{MnO}_{3}$ Einkristallen gefunden werden [31]. Der Einfluss der Verspannung der Struktur durch die Fehlanpassung der A-Platz Ionen und deren Unordnung auf die Austauschwechselwirkung (hier DE) ist ersichtlich anhand der Diskrepanz zwischen der theoretischen und tatsächlichen Temperatur. Ein Ansatz zur Erhöhung der Übergangstemperatur mittels einer Verminderung von $\sigma^{2}$ wird in Kap. 4.2 vorgestellt.

Auch in den ferro- und ferrimagnetischen Doppelperowskiten, mit zwei unterschiedlichen Atomsorten auf den B-Plätzen, ist die Kationen-Ordnung entscheidend für die magnetischen Eigenschaften. Diese Materialien haben gemein, dass die gleiche B-Platz Sorte antiferro- 
2 Grundlagen zur elektronischen Struktur der Perowskite

magnetisch koppelt, während ungleiche B-Platz Ionen ferromagnetisch koppeln. Auf diese Mechanismen wird in Kap. 5 näher eingegangen. 


\section{Experimentelle Methoden}

\subsection{Metallorganische Aerosol Deposition}

\subsubsection{Grundprinzip}

Die Metallorganische Aerosol Deposition (MAD) [13] ist eine vakuumfreie chemische Depositions-Methode zur Herstellung von dünnen metalloxidischen Filmen. Metallorganische Verbindungen (Präkursoren) werden in einem organischen Lösungsmittel (meist N,N-Dimethylformamid, vk. DMF) gelöst und mit trockener Druckluft fein zerstäubt. Es entsteht ein Aerosol mit einer Tröpfchengröße von $20-50 \mu m$, das auf ein heißes oxidisches Substrat gesprüht wird. In der Nähe des Substrates findet eine Oxidation (in Gleichung ((5)) anhand von Manganacetylacetonat) statt bei der, neben gasförmigen $\mathrm{CO}_{2}$ und $\mathrm{H}_{2} \mathrm{O}$, ein Metalloxid entsteht. Dieses kann sich auf der Substratoberfläche epitaktisch anlagern, während die Abgase abgepumpt werden.

$$
\mathrm{Mn}\left(\mathrm{C}_{5} \mathrm{H}_{7} \mathrm{O}_{2}\right)_{3}+18.25 \mathrm{O}_{2} \longrightarrow \mathrm{MnO}_{2}+15 \mathrm{CO}_{2}+10.5 \mathrm{H}_{2} \mathrm{O}
$$

Die spätere stöchiometrische Zusammensetzung der metalloxidischen Schicht wird über die Konzentration der Präkursoren in der Lösung bestimmt. Dabei muss jedoch beachtet werden, dass diese Verhältnisse nicht exakt den Metallionenverhältnissen in der Metalloxidschicht entsprechen. Die Ursache für diese Diskrepanz wurde bisher noch nicht offen gelegt, was vor allem an dem komplexen Zusammenspiel zwischen Kinematik und Thermodynamik während der Oxidation der verschiedenen Präkursoren (und des organischen Lösungsmittels) und dem Wachstum der dünnen Schicht liegt. Jedoch lassen sich stabile empirische Faktoren $\nu_{i / j}$ zwischen der Konzentration zweier Präkursoren $i$ und $j$ in der Lösung finden, sodass das Verhältnis der Stoffmenge der entsprechenden Metalle in dem Metalloxid äquivalent ist. Über Methoden, wie die Energiedispersive Röntgenspektroskopie (EDX) oder die Massenspektrometrie mit induktiv gekoppelten Plasma (ICPMS), lassen sich diese Faktoren nur ungenau und nicht reproduzierbar ermitteln. Das liegt vor allem daran, dass die Masse und die Schichtdicke des Filmes wesentlich kleiner sind als die des Substrates, wodurch der Substrathintergrund der Messung das Ergebnis verfälscht. Aus diesem Grund wurden die Faktoren in dieser Arbeit durch die Präparation und Charakterisierung von zwei- bzw. dreikomponentigen Sys-temen bestimmt. In der Regel zeigen die physikalischen Eigenschaften der komplexen Oxide eine starke Abhängigkeit von ihrer Stöchiometrie. Unter Kenntnis der genauen strukturellen, elektrischen und magnetischen Eigenschaften des zu deponierenden 
Materials, können die Faktoren ermittelt werden. Die Einstellung der $\nu_{i / j}$ Faktoren muss natürlich für jedes zu deponierende, mehr-komponentige Material, sowohl auch für eine neue Präkursor-Charge geschehen ${ }^{2}$. Zusätzlich können diese Faktoren eine Abhängigkeit von weiteren Komponenten in der Lösung, vom Substratmaterial und von der Depositionstemperatur zeigen. Die einzelnen Stoffmengen, die für mehr-komponentige Systeme benötigt werden, können über:

$$
n_{i}=x_{j / i} \cdot n_{j} \cdot \nu_{i / j}
$$

bestimmt werden, wobei $n$ die Stoffmengen der Präkursoren $i$ und $j$ und $x_{j / i}$ das gewünschte Verhältnis der Stoffmengen der Metalle der Präkursoren j zu i in dem Metalloxid sind. Auf die Einstellung der $\nu_{i / j}$ für die, in dieser Arbeit präparierten, Perowskite wird in den entsprechenden Kapiteln zu den Materialien eingegangen.

Im Rahmen dieser Arbeit wurden drei verschiedene Ausführungen der MAD für die Probenpräparation eingesetzt. Alle haben gemein, dass das metallorganische Aerosol von einer pneumatischen Multikanal-Düse derselben Bauart erzeugt und das Substrat resistiv durch einen dotierten Siliziumkarbid-Heizer erwärmt wird. In den folgenden Unterkapiteln wird auf zwei verschiedenen MAD-Ausführungen näher eingegangen (die dritte Ausführung wird ausführlich in Kap.5.2.3 vorgestellt). Das letzte Unterkapitel beschäftigt sich mit den verwendeten Substraten. Sofern nicht anders aufgeführt, wurden alle in dieser Arbeit vorgestellten Filme mit einer Depositionsrate von $5-10 \mathrm{~nm} / \mathrm{min}$ bei einer Heizertemperatur von $980^{\circ} \mathrm{C}$ gewachsen.

\subsubsection{Standard MAD (MAD-1)}

In der Standard Ausführung der MAD, schematisch dargestellt in Abb.6 a), wird die Lösung mit Hilfe von zwei peristaltischen Präzisions- $\mu l$ Pumpen des Typs Reglo Digital von der Firma Ismatec befördert. Durch einen He-Ne-Laser der auf eine Stelle knapp unter der pneumatischen Düse fokussiert ist und einer Photodiode, außerhalb dieses Strahlenganges, wird eine zeitliche Flussmessung realisiert. Sobald Aerosole aus der pneumatische Düse austreten, streuen Sie das Laserlicht, das von der Photodiode als Signal aufgenommen wird. Damit lässt sich, zusammen mit der Flussrate der peristaltischen Pumpen, eine indirekte Schichtdickenkontrolle realisieren. Jedoch kann der Reproduktionsfehler der Flussrate bei einem Wert von bis $\mathrm{zu} 10 \%$ liegen $^{3}$. Folglich ist dieser relative Fehler auch eine minimale Abschätzung für

\footnotetext{
${ }^{2}$ Verschiedene Präkursor Chargen können einen unterschiedlichen Verunreinigungsgrad von anderen organischen Komponenten oder einen unterschiedlichen Wasseranteil aufweisen, was zu einer abweichenden Effizienz während der Deposition führt.

${ }^{3}$ Die Flussrate kann sich bei zwei direkt aufeinanderfolgenden Depositionen um diesen Wert unterscheiden.
} 
den Fehler des aus der Depositionszeit abgeschätzten Volumen der deponierten Lösung und somit der Schichtdicke. Für die Deposition größerer Volumina eignet sich die Abmessung des Volumens durch Präzisionspipetten. In dieser MAD Anlage kann die Heizertemperatur mit einem Zwei-Wellenlängen-Pyrometer für Temperaturen $>800^{\circ} \mathrm{C}$ überwacht werden.

\subsubsection{MAD mit in situ Wachstumskontrolle (MAD-3)}

Die zweite MAD Variante, die in dieser Arbeit genutzt wurde, ist ausgestattet mit einer in situ ellipsometrischen Überwachung der Deposition und vollautomatischen Hochpräzisionspumpen für die Injektion der Metallorganischen Lösung. Der schematische Aufbau dieser Anlage ist in Abb.6 b) dargestellt. Das Aerosol wird mit Hilfe einer pneumatischen Düse erzeugt, die mit bis zu 6 verschiedenen metallorganischen Lösungen und Druckluft gespeist wird. Die Lösung wird in dieser Anlage durch die Hochpräzisions-Spritzendosierern SyrDos 2 von der HiTec Zang GmbH transportiert. Die Präzision der geförderten Menge hängt von dem Volumen der installierten Spritze ab. Während der gesamten Arbeit wurden $500 \mu l$-Spritzen benutzt, wodurch eine Präzision von $10 \mathrm{nl}$ abgeschätzt werden kann. Das typische Volumen, das man für das Wachstum einer Perowskit-Monolage (ML) benötigt, liegt bei 5 - 10 $\mu$ l. Somit kann die Dicke, der zu wachsenden Schicht, rechnerisch auf 1/100 ML genau eingestellt werden. Mit Hilfe eines sogenannten aktiven Shutters können die Leitungen mit metallorganischer Lösung befüllt werden, ohne das während der Befüllung bereits eine Deposition stattfindet. Dieser aktive Shutter ist mit einer zusätzlichen Druckluftdüse ausgestattet, um die Kühlung des Heizers durch Druckluft weiterhin, auch nach dem Abschatten von der Depositionsdüse, zu gewährleisten. Mit dieser Ausstattung eignet sich diese MAD für die Präparation von präzisen Heterostrukturen.

Der ellipsometrische Aufbau registriert die Änderung der optischen Brechungsindices der Filmoberfläche während der Deposition des Filmes durch die Änderung der Reflexionskoeffizienten. Dieser Aufbau wurde nach dem Polarisator-Modulator-Probe-Analysator-Prinzip (engl. polarizer-modulator-sample-analyzer, vk. PMSA) [33, 34], wie in Abb.6 b) dargestellt, realisiert. Für die Maximierung des Messsignales bei einer Änderung des Verhältnisses der Reflexionskoeffizienten $\rho=r_{p} / r_{s}$, mit den komplexen senkrechten bzw. parallelen Reflexionskoeffizienten $r_{s}$ bzw. $r_{p}$, wurde der He-Ne-Laser mit einem Winkel von $25^{\circ}$ zur Substratoberfläche ausgerichtet. Der Komplementärwinkel davon entspricht in etwa dem Brewster-Winkel von $\mathrm{SrTiO}_{3}$ [35], dem am häufigsten verwendeten Substrat. Die Polarisationsfilter sind auf einen Winkel von $45^{\circ}$ eingestellt und die Modulation des polarisierten Lichtes wird von einem Photoelastischen Modulator (PEM) mit der Frequenz $\omega=50 k \mathrm{~Hz}$ übernommen. In 
a) MAD-1

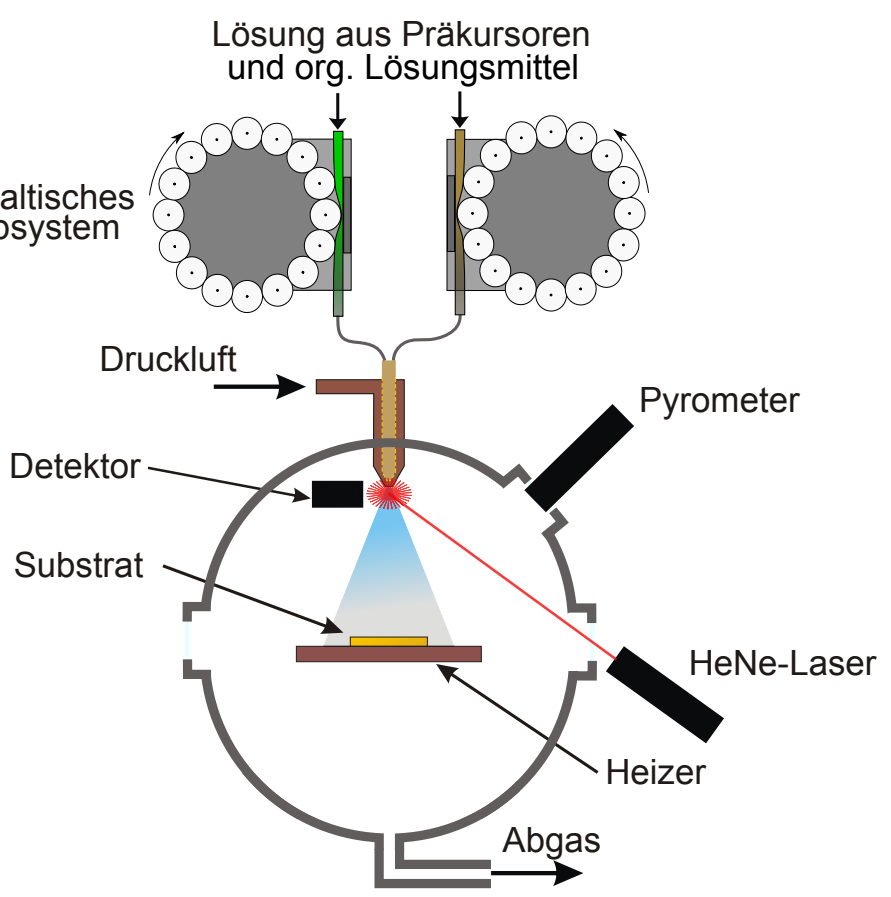

\section{b) MAD-3}

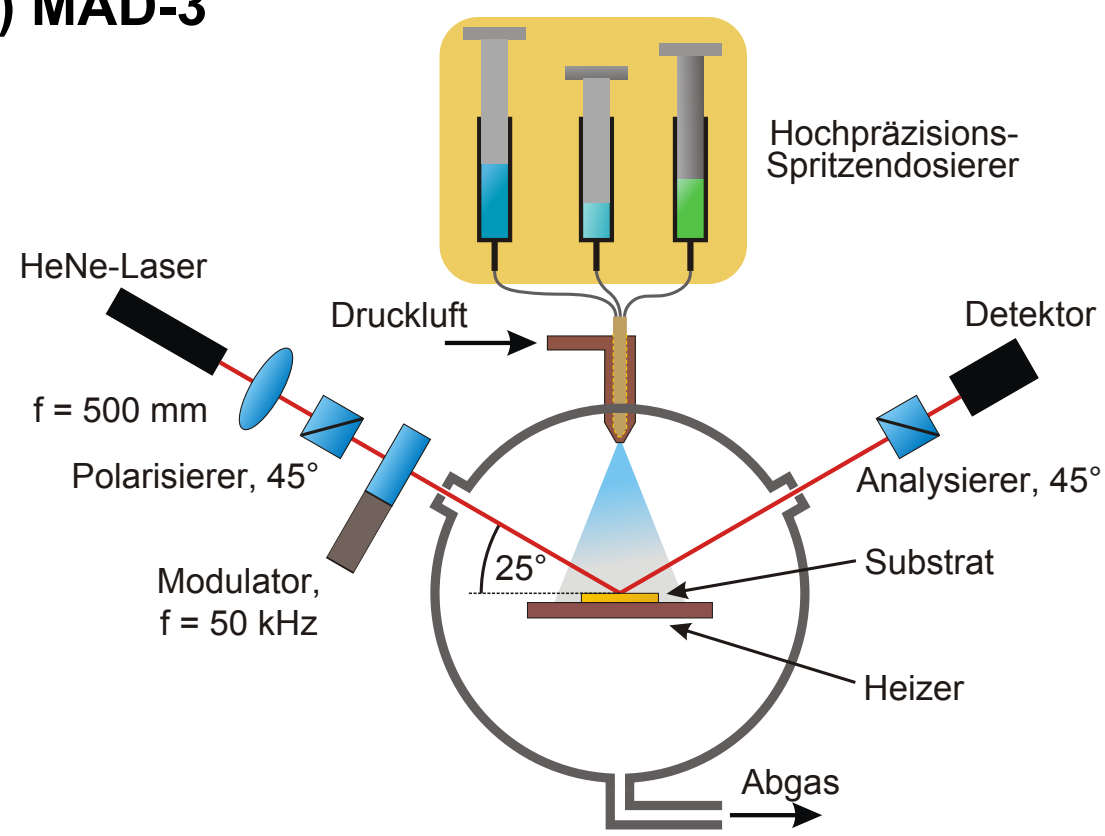

Abbildung 6: Schematischer Aufbau der a) MAD-1 und b) MAD-3 Anlage. Während in der MAD1 (a) die Lösung über eine peristaltische Pumpe transportiert wird und die Depostionsüberwachung über eine Flussmessung (Streulicht eines He-Ne-Lasers durch Aerosole) erfolgt, ist dies in der MAD3 über einen Hochpräzisions-Spritzendosierer bzw. über ein ellipsometrisches PMSA-Setup gelöst. Sowohl Heizer als auch pneumatische Düse sind in beiden Anlagen baugleich. Abbildung b) ist eine modifizierte Version aus der Literatur [32]. 
diesem Aufbau wird die Amplitude der Modulation des PEMs so gewählt, dass die von dem PEM generierte Phasenverschiebung $\delta$ zwischen der parallelen und senkrechten Komponente der Lichtwelle $139^{\circ}$ beträgt. Bei diesem Winkel ist die Bessel-Funktion nullter Ordnung $J_{0}\left(\delta=139^{\circ}\right) \sim 0$. Die erste und zweite Harmonische $I_{\omega}$ bzw. $I_{2 \omega}$ und die DC Lichtintensität wird über einen Photodetektor mit der Hilfe von zwei Lock-in-Verstärkern und einem Keithley 2000 Multimeter gemessen. Die beiden AC-Intensitäten sind mit der Voraussetzung $J_{0}(\delta)=0$ über den Zusammenhang $I_{\omega}=J_{1}(\delta) \sin (2 \Psi) \sin (\Delta)$ bzw. $I_{\omega}=J_{2}(\delta) \sin (2 \Psi) \cos (\Delta)$ mit dem Polarisations-Rotationswinkel $\Psi$ und der Phasenverschiebung $\Delta$, zwischen der einfallenden und reflektierten Lichtwelle, verknüpft. $J_{1}(\delta)$ und $J_{2}(\delta)$ sind dabei die BesselFunktionen der ersten und zweiten Ordnung. Diese beiden Winkel sind wiederum über $\rho=\tan (\Psi) \exp (i \Delta)$ mit dem Verhältnis der Reflexionskoeffizienten verknüpft [33].

Diese Überwachungstechnik ermöglicht eine in situ Schichtdickenkontrolle, über die Interferenz an dünnen Schichten [33] und/oder Oszillationen die bei Lage-für-Lage Wachstum beobachtet werden können, [36-39] für den MAD Prozess bei Umgebungsdruck. Darüber hinaus gelingt damit z.B. auch die in situ Observation der stöchiometrischen Koeffizienten bei einer homoepitaktischen Deposition oder auch die in situ Bestimmung des Valenzzustandes von Mangan bei der Deposition von Manganat-Multilagensystemen [32, 36]. Die mannigfaltige Anwendbarkeit dieser Überwachungsmethode wird ausführlich in der Dissertation von Markus Jungbauer behandelt [36].

In Zusammenarbeit mit Markus Jungbauer wurde die Software „MAD Commander“ erstellt, die den Depositionsprozess steuert und überwacht. Die Software übernimmt die Pumpenansteuerung, die Heizer-Ansteuerung und die Aufnahme und Vorverarbeitung der ellipsometrischen Messung.

\subsubsection{Die Substrate}

In dieser Arbeit wurden einkristalline und atomar polierte $M g O$ Substrate mit (001) Orientierung, $\mathrm{Al}_{2} \mathrm{O}_{3}$ Substrate mit (0001) Orientierung und $\mathrm{SrTiO}_{3}$ (STO) Substrate mit den Orientierungen (001) und (111) verwendet. Alle Substrate waren auf die Größe 10·5・0.5 $\mathrm{mm}^{3}$ zugeschnitten und einseitig poliert. Diese Substrate wurden von der Crystal GmbH und der CrysTec GmbH bezogen und in der Zwischenzeit trocken in einem Exsikkator gelagert. Die relevanten physikalischen Eigenschaften der Substrate werden im nächsten Kapitel behandelt.

Die STO Substrate wurden einer zusätzlichen Vorbehandlung unterzogen, die zu einer ein- 
heitlichen Terminierung der Substratoberfläche führt. Im Gegensatz zu $\mathrm{MgO}$ und $\mathrm{Al}_{2} \mathrm{O}_{3}$ ist das STO aus zwei Metalloxiden $\left(\mathrm{SrO}\right.$ und $\mathrm{TiO}_{2}$ ) aufgebaut, die sich lagenweise in der [100]Richtung abwechseln. Dies hat zur Folge, dass die Oberfläche der STO Substrate sowohl mit $\mathrm{SrO}$ als auch $\mathrm{TiO}_{2}$ abgeschlossen wird. Um die Beeinflussung einer solchen arbiträren Substrat-Terminierung auf das Wachstum des dünnen Films zu verhindern, wurde die Oberfläche auf Ti-Oxid terminiert. Bereits 1994 gelang Kawasaki et al. [40] die Terminierung von STO(001) auf eine $\mathrm{TiO}_{2}$-Oberfläche durch chemisches Ätzen mit einer, aus der Halbleiterindustrie bekannten, gepufferten Flusssäure und anschließendem thermischen Auslagern. Dieses Verfahren wurde später von Koster et al. [41] noch weiter optimiert und von Biswas et al. [42] auch für andere STO Orientierungen gezeigt. Für diese Arbeit wurde das Verfahren, wie in [42] beschrieben verwendet: Zunächst wurden die Substrate für 10min in deionisiertem Wasser mit der Hilfe eines Ultraschallbades gewaschen. Anschließend wurden sie für $30 \mathrm{~s}$ in einer kommerziellen Flusssäure-Puffer-Lösung $\left(\mathrm{NH}_{4} F-H F\right.$, CAS Nummer 70456 - 74 5) geätzt. Nach einem weiteren Wasch- und Abspülvorgang in deionisiertem Wasser wurden die Substrate auf dem Siliziumkarbid-Heizer der MAD 1 in der Umgebungsatmosphäre für $1 h$ bei $\sim 1000^{\circ} \mathrm{C}$ ausgelagert.

\subsection{Charakterisierungsmethoden}

Dieses Kapitel gibt einen Überblick und eine kurze Einführung in die Methoden zur strukturellen, magnetischen und elektrischen Charakterisierung der dünnen Filme und Überstrukturen, die in dieser Arbeit verwendetet wurden. Dabei wird vor allem auf die Aufarbeitung und die Analyse der experimentellen Daten eingegangen. Zusätzlich werden die Resultate der jeweiligen Messverfahren an den, in dieser Arbeit verwendeten, Substraten präsentiert. Die Kenntnis der physikalischen Eigenschaften der Substrate ist absolut notwendig, um die Messungen an den dünnen Filmen korrekt zu interpretieren.

\subsubsection{Rastersondenmikroskopie}

Die Rastersondenmikroskopie ist eine Methode zur Bestimmung der Oberflächenmorphologie von dünnen Schichten. In dieser Arbeit wurde sowohl die Rasterkraftmikroskopie (SFM, Apparaturen: Innova-AFM von Bruker, Nanoscope AFM-Option von Veeco Instruments) als auch die Rastertunnelmikroskopie (STM, Apparaturen: Nanoscope STM-Option von Veeco Instruments) verwendet. Bei der Rastersondenmikroskopie wird eine physikalische Größe an der Sonde, die nahe oder auf der Probenoberfläche ist, observiert, die eine direkte 

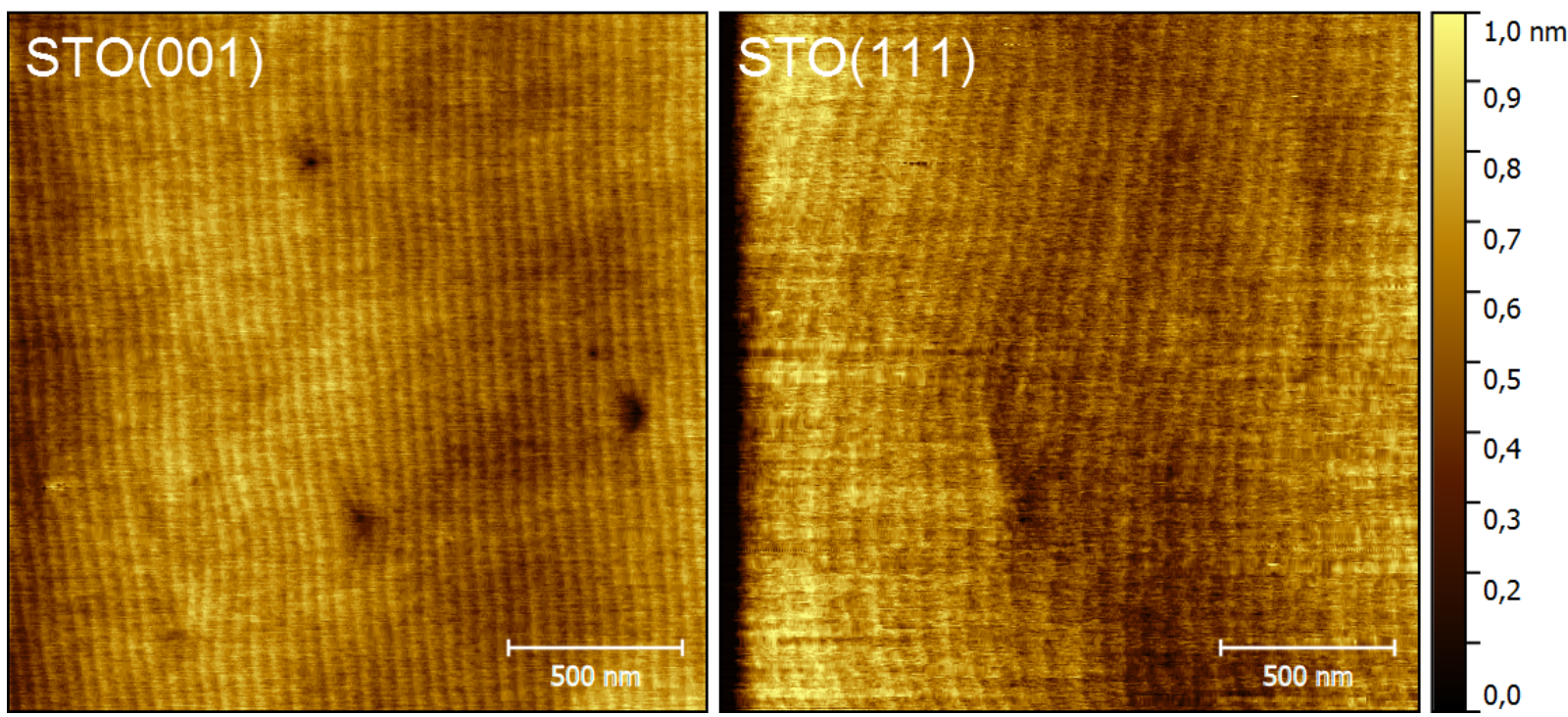

Abbildung 7: SFM Aufnahmen von einem STO(001) und STO(111) Substrat nach der in Kap. 3.1.4 beschriebenen Vorbehandlung.

Abhängigkeit zum Sonden/Proben-Abstand zeigt. Im Falle der STM ist dies ein Tunnelstrom zwischen einer metallische Spitze und der Probe, während es bei der SFM die Auslenkung eines Cantilevers durch den Kontakt zur Probenoberfläche ist. Diese physikalische Größe wird über einen Proportional-Integral-Regler (PIC-Kreis) konstant gehalten, während die Sonde mit der Hilfe von piezoelektrischen Stellelementen über die Probe geführt wird. Die Änderung die in den PIC-Kreis eingehen, um die Größe konstant zu halten, liefern einen direkten Rückschluss auf die Oberflächenmorphologie der Probe. Für weitergehende Informationen über diese Methode sei auf die Literatur [43] verwiesen. Alle in dieser Arbeit gezeigten Bilder aus der Rastersondenmikroskopie wurden mit der Software Gwyddion [44] bearbeitet: Zunächst wurden die Messzeilen aus den Rohdaten durch das Abgleichen des Höhenmedians korrigiert, anschließend wurden fehlerhafte Messzeilen ausgeglichen und die Daten durch die Subtraktion der Mittelwertebene nivelliert.

In Abb.7 ist die Oberflächenmorphologie für zwei STO Substrate mit (001) und (111) Orientierung nach der Vorbehandlung, wie sie in Kap.3.1.4 beschrieben ist, gezeigt. Aufgrund des hohen Widerstandes von STO bei Raumtemperatur wurden diese Aufnahmen mit dem SFM erstellt. Auf den Bildern sind klar Stufen von der Höhe einer Einheitszelle für die (001) bzw. einer Einheitszelle $/ \sqrt{3}$ für die (111) Orientierung. Diese Stufen resultieren aus dem Fehlschnitt zwischen der Substratoberfläche und den kristallinen Achsen. Auf den Stufen ist die Oberfläche atomar glatt. Aus dieser Messung lässt sich somit die Vizinalität der Substrate gewinnen. Sie liegt für die in Abb.7 gezeigten Substrate bei $0.44^{\circ}$ für das STO(001) und bei $0.2^{\circ}$ für das STO(111). Generell ist die Vizinalität für alle in dieser Arbeit verwendeten 
Substrate $<0.5^{\circ}$. In beiden Bildern sind auch Stufen zu erkennen, die plötzlich verschwinden bzw. auftauchen. Dies sind Versetzungsfehler in der Kristallstruktur der Substrate, die sich bis auf die Oberfläche fortsetzen.

\subsubsection{Röntgendiffraktion}

Die Röntgendiffraktion (XRD) ist ein Verfahren um die strukturellen Eigenschaften von kondensierter Materie zu bestimmen. Dies geschieht mittels der Streuung elektromagnetischer Wellen. Da typische Atomgrößen bzw. -abstände im Å-Bereich liegen, muss Röntgenstrahlung verwendet werden. Das eingestrahlte Röntgenlicht wird dabei an den Elektronenhüllen der Atome der Probe gestreut. Im Kristall interferiert das gestreute Röntgenlicht. Im Folgenden sei $\vec{k}_{i}\left(\vec{k}_{s}\right)$ der Wellenvektor der einfallenden (gestreuten) Röntgenstrahlung. Wird die Laue-Bedingung $\vec{k}_{s}-\vec{k}_{i}=\vec{G}_{h k l}$ erfüllt, wobei $\vec{G}_{h k l}$ der reziproke Gittervektor ist, der die Netzebenenschar (hkl) repräsentiert, findet eine konstruktive Interferenz statt. Vereinfacht lässt sich dieser Sachverhalt in der Bragg-Bedingung $n \lambda=2 \cdot d_{h k l} \cdot \sin (\theta)$ wiederfinden, wobei $\theta$ der halbe Winkel zwischen den Wellenvektoren $\vec{k}_{i}$ und $\vec{k}_{s}, d_{h k l}=2 \pi /\left|\vec{G}_{h k l}\right|$ der Abstand der Netzebenen der Netzebenenschar (hkl), $\lambda$ die Wellenlänge der Röntgenstrahlung und $n$ eine natürliche Zahl ist. Die Intensität des Reflexes zur Ebenenschar (hkl) $I_{h k l}$, ist proportional zu dem Strukturfaktor

$$
F_{h k l}=\sum_{n=1}^{N} f_{n} \exp \left(2 \pi i\left(\vec{G}_{h k l} \vec{r}_{n}\right)\right.
$$

wobei $N$ die Anzahl der Atome mit den atomaren Streufaktoren $f_{n}$ an der Position $\vec{r}_{n}$ in der Einheitszelle ist. Für die absolute Intensität müssen noch alle Einheitszellen der Anzahl $M$ berücksichtigt werden, die vom Röntgenlicht bestrahlt werden: $\sum_{m=1}^{M} \exp \left(i \vec{G}_{h k l} \vec{R}_{m}\right)$, wobei $\vec{R}_{m}$ die Position der Einheitszelle $m$ ist. Dieser Faktor beschreibt auch das Auftreten von Laue-Oszillationen (Intensitätsoszillationen neben dem Bragg-Reflex) bei dünnen Schichten, aus denen sich die Schichtdicke bestimmen lässt. Zusätzlich muss auch die Polarisierbarkeit von Röntgenlicht und die Bewegung der Probe während der Messung einkalkuliert werden, was mit dem Lorentz-Polarisations-Faktor $\left(1+\cos ^{2}(2 \theta)\right) /\left(\sin ^{2}(\theta) \cos (\theta)\right)$ geschieht. Die Gesamtintensität ergibt sich aus dem Betragsquadrat der genannten Faktoren [45] zu:

$$
I_{h k l}=\left|F_{h k l} \sum_{m=1}^{M} \exp \left(i \vec{G}_{h k l} \vec{R}_{m}\right)\left(1+\cos ^{2}(2 \theta)\right) /\left(\sin ^{2}(\theta) \cos (\theta)\right)\right|^{2}
$$

Diese Vorbetrachtung zeigt, dass in den XRD-Messungen eine enorme Informationsdich- 


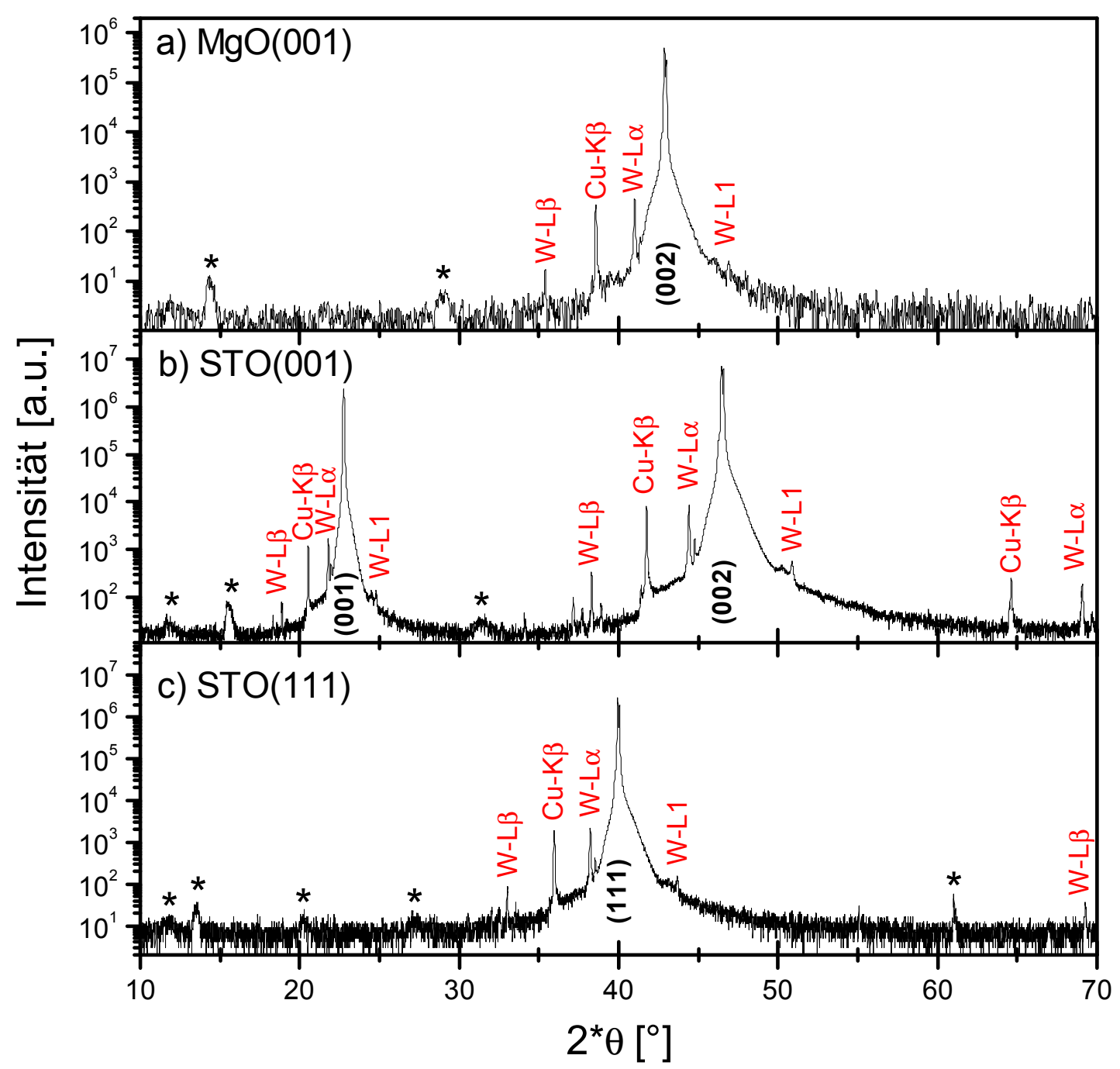

Abbildung 8: XRD-Messung der in dieser Arbeit verwendeten Substrate: a) $M g O(001)$, b) STO(001) und c) STO(111). Neben der Hauptwellenlängen $C u-K \alpha 1,2$ können auch andere Wellenlängen von $C u$ und $W$ beobachtet werden (in rot indiziert). Kleine unidentifizierbare Reflexe sind mit * markiert. 


\section{Experimentelle Methoden}

te über die strukturellen Eigenschaften steckt. Aufgrund der Tatsache, dass Detektoren die Intensität als das Betragsquadrat der eingehenden Faktoren messen, geht die Phaseninformation verloren. Diese würde man benötigen, um aus den Messdaten direkt die strukturellen Eigenschaften bestimmen zu können (Rücktransformation der Daten zu einem passenden strukturellen Modell). Aus diesem Grund muss, um die Daten auswerten zu können, eine XRD-Simulation für ein strukturelles Modell erstellt werden und mit den Messdaten abgeglichen werden. Dieses Modell wird nun weiter angepasst, bis die simulierten und gemessenen Daten eine zufriedenstellende Übereinstimmung erreichen. Von Markus Jungbauer wurde eine Simulation erstellt, die über Gleichung (8) das XRD-Pattern für perowskitische Übergitter simuliert. Zusätzlich wurde neben den Faktoren aus Gleichung (8) die Temperaturabhängigkeit über den Debye-Waller-Faktor $\exp \left(-1 / 3\left|\vec{G}_{h k l}\right|^{2} \overline{u^{2}}(T)\right)$, eingebracht, wobei $u(T)$ die temperaturabhängige Oszillationsamplitude der Atome ist, die über das DebyeModell abgeschätzt werden kann. Für die Simulationen wurden die atomaren Streufaktoren aus [46] verwendet. Im Rahmen dieser Arbeit wurde die Simulation für die Analyse von Antiphasen-Defekten in Doppelperowskiten erweitert, wodurch die B-Platz Ordnung in den Doppelperowskiten über die Röntgendiffraktion abgeschätzt werden kann.

In dieser Arbeit wurde das Röntgendiffraktometer D8 Advance der Firma Bruker AXS verwendet. Als Röntgenquelle diente eine Kupferanode, deren Strahlung mittels eines GöbelSpiegels auf die $C u-K_{\alpha_{1,2}}$-Linien monochromatisiert wurde. Das gewichtete Mittel dieser Strahlung, das die Intensität der Wellenlängen berücksichtigt, liegt bei $\lambda=0.154184 \mathrm{~nm}$. Die Messungen wurden in der Bragg-Brentano-Geometrie $(\theta-2 \theta$-Geometrie) durchgeführt.

In Abb.8 sind die Weitwinkel XRD Messungen für die, in dieser Arbeit, wichtigsten Substrate dargestellt. Aufgrund des Auftretens von zahlreichen Fremdreflexen werden diese Daten bei allen weiteren XRD Spektren unterlegt sein, um die Reflexe des dünnen Filmes klar zu erkennen. Die Spektren zeigen deutlich, dass die Monochromatisierung des Röntgenlichtes nicht vollständig geschieht. Einige dieser Fremdreflexe können weiteren Röntgenwellenlängen von $C u$ oder $W$ zugeordnet werden (rot in Abb.8), während andere nicht zugeordnet werden können (markiert mit * in Abb.8). Etwaige Reflexe der Schichten von anderen Wellenlängen (ausgenommen den $C u-K_{\alpha_{1,2}}$-Linien) sind aufgrund der großen Volumendifferenz zwischen Film und Substrat (und damit Intensitätsdifferenz) nicht zu erwarten.

\subsubsection{Röntgenreflexion}

Für Winkel $2 \theta<10^{\circ}$ und Röntgenwellenlängen wie im vorhergehenden Kapitel ist die Laue-Bedingung für Perowskite nicht erfüllt. Jedoch kann hiermit die Bragg-Bedingung 
$n \lambda=2 \cdot d \cdot \sin (\theta)$ für typische Schichtdicken $d$ von dünnen Filmen erfüllt werden. In der Messung kann eine Oszillation mit $n$ Ordnungen an der Flanke des Totalreflexionspeaks observiert werden. Bei diesen Messungen spricht man von der Röntgenreflexion (XRR), die in dieser Arbeit auch mit dem Röntgendiffraktometer D8 Advance der Firma Bruker AXS durchgeführt wurden. Neben der Bestimmung der Schichtdicke erhält man aus diesen Messungen auch Informationen über die Rauigkeit der Filmoberfläche und an der Substrat-Film Grenzfläche. Letzteres beschreibt die chemische Interdiffussion zwischen Film- und Substratmaterial. Für Multilagensysteme gehen in die Messdaten auch die Information über die Rauigkeiten aller Grenzflächen und die Schichtdicken aller Lagen ein. Genau wie bei den XRD Messungen ist hier auch das Phasenproblem vorhanden und damit muss auch die gleiche Prozedur bei der Interpretation dieser Messergebnisse durchlaufen werden. Für einphasige, dünne Schichten kann das XRR Spektrum über die Fresnelschen Formeln berechnet [47] und für kompliziertere Multilagensysteme z.B. über den Parratt-Formalismus iterativ simuliert werden $[48,49]$. Voraussetzung für die Erstellung eines theoretischen Spektrums ist die Kenntnis der komplexen optischen Konstanten $N(\lambda)=1-\delta(\lambda)+i \beta(\lambda)$ des Substrat- und Schichtmaterials bei der entsprechenden Röntgenwellenlänge $\lambda$. Die reelle (imaginäre) optische Konstante $\delta(\beta)$ beschreibt die Reflexion (Absorption) und kann aus den Streuamplituden der Elemente bestimmt werden, die in der Literatur beispielsweise in der Chantler Tabelle [50] gefunden werden können. Die erwähnten Methoden werden von der Software ReMagX [51], welche in dieser Arbeit verwendet wurde, aufgegriffen um (unter anderem) XRR-Spektren zu simulieren bzw. an Messdaten anzupassen. Diese Software wurde von der Gruppe um E. Goering vom Max-Planck-Institut für Intelligente Systeme in Stuttgart entwickelt [51]. Die Grenzflächenrauigkeit wird in dieser Software über die Nevot-Croce-Methode modelliert [52]. Bei dieser Methode werden die optischen Konstanten der angrenzenden Materialien über eine gaußsche Fehlerfunktion ineinander überführt, wobei das quadratische Mittel (RMS) der Rauigkeit $\sigma$ die charakteristische Größe der Breite des Überganges ist.

\subsubsection{Transmissionselektronenmikroskopie}

Die in dieser Arbeit gezeigten Transmissionselektronenmikroskopie (TEM) Messungen, wurden in der Gruppe um Johan Verbeeck vom EMAT Institut der Universität von Antwerpen durchgeführt und analysiert. Die TEM Technik erlaubt die bildliche Charakterisierung von Materialien auf der atomaren Größenskala. Dazu wird eine, auf wenige $n m$ dicke, Probe von einem Elektronenstrahl, der von einer Elektronenkanone erzeugt und von elektromagnetischen Linsen fokussiert wird, beleuchtet. Die Wechselwirkung zwischen den hochenergetischen Elektronen aus dem Strahl und den Atomen, Phononen bzw. dem Elektronensystem 
der Probe führen zu elastischen und inelastischen Streuprozessen, die auf der atomaren Skala Auskunft über die Chemie und die Struktur der Probe liefern. Weiter sollen in diesem Unterkapitel zusammenfassend spezielle TEM-Verfahren vorgestellt werden, die für diese Arbeit eine wichtige Rolle gespielt haben.

\section{High angle annular dark field scanning TEM}

Bei dem Raster-TEM Verfahren (STEM, $\mathrm{S}=$ scanning) wird die Probe mit einem fokussierten Elektronenstrahl abgerastert, um ortsabhängige Informationen zu erhalten. Hierbei kann eine Ortsauflösung von 0.05nm erreicht werden [53]. In dem high angle annular dark field Modus (HAADF) werden nun die Elektronen registriert, die um 50 - 250mrad, in Bezug zum einfallenden Elektronenstrahl, von den Atomen der Probe gestreut wurden. Die Streuung für diese Elektronen wird als Rutherford-artig angesehen [54]. Die gemessene Intensität am HAADF Detektor $I_{H A A D F}$ und damit der Kontrast einer HAADF Aufnahme ist damit exponentiell abhängig von der Ordnungszahl $Z$ der Atome mit $I_{H A A D F} \sim Z^{1.6-1.9}$ [55]. Mit diesem Verfahren gelingt die Abbildung der Struktur einer Probe, welche einen Kontrast proportional zur Masse der abgebildeten Atome hat.

\section{Elektronenenergieverlustspektroskopie}

Die Elektronenenergieverlustspektroskopie (EELS) ist ein STEM Verfahren bei dem das Spektrum über die kinetischen Energie der inelastisch gestreuten Elektronen erstellt wird. Üblicherweise werden dabei die Elektronen detektiert, die 0 - 100 mrad, in Bezug zum einfallenden Elektronenstrahl, gestreut werden. Der Energieverlust $\Delta E$ der Elektronen kann in zwei Bereiche unterschieden werden: Niedrig-Verlust-Bereich (low-loss spectra) mit $\Delta E \leq$ $50 \mathrm{eV}$ und dem Kern-Verlust-Bereich (core-loss spectra) mit $\Delta E \sim 50-3000 \mathrm{eV}$. In dem Kern-Verlust-Bereich sind die Elektronen zu finden, die an den Elektronen der inneren Schale der Atome inelastisch gestreut wurden. Bei dieser Streuung werden gebundene Elektronen aus der inneren Schale der Atome durch das hochenergetische Elektron angeregt und in einen höheren unbesetzten Zustand befördert. Diese Intrabandübergänge sind über ihre Anregungsenergien charakterisierbar, die von der Art und dem Zustand des Atoms und von dem Quantenzustand des gebundenen Elektrons abhängen. Beispielsweise hat die Anregung eines $2 p$ Elektrons des Mangans in die $3 d$ Schale eine charakteristische Energie von $640 \mathrm{eV}$. Dieser Übergang wird auch $M n L_{2,3}$ genannt. Über einen breiten Spektralbereich kann somit eine EELS Feinstruktur erstellt werden, die Auskunft über die Art und den Valenzzustand des durch das STEM selektierte Atom liefern kann. Für weitergehende Details sei auf die Literatur verwiesen $[53,56]$. 


\section{Energiedispersive Röntgenspektroskopie}

Rekombinieren die durch inelastische Stöße angeregten Löcher in den inneren Schalen mit höherenergetischen Elektronen, werden charakteristische Röntgen-Photonen emittiert. In der energiedispersiven Röntgenspektroskopie wird ein Energiespektrum dieser Röntgenstrahlung aufgenommen. Diesem Spektrum kann eindeutig ein Element zugeordnet werden. Im STEM Verfahren ist es mittlerweile möglich, wie in der EELS, atomar aufgelöste, elementspezifische Karten zu erstellen [57].

\subsubsection{Raman-Spektroskopie}

Bei der Raman-Spektroskopie [58] nutzt man die inelastische Streuung von monochromatischem sichtbaren Licht an den optischen Phononen eines Festkörpers, um die strukturelle Eigenschaften des untersuchten Materials zu bestimmen. Da das Phononenspektrum von der Kristallstruktur und der Masse der Atome abhängt, kann über das Raman-Spektrum ein unbekanntes Material identifiziert werden. Innerhalb dieser Arbeit wurde die RamanSpektroskopie genutzt um den Oxidationszustand von Eisenoxid-Dünnfilmen zu bestimmen. Für weiterführende Informationen bezüglich dieser Methode sei auf die Literatur verwiesen $[58]$.

\subsubsection{SQUID Magnetometrie}

Die Messungen der magnetischen Eigenschaften der dünnen Filme wurden, in dieser Arbeit, größtenteils mit der RSO-Option des Magnetic Properties Measurement System (MPMS) von der Firma Quantum Design durchgeführt. Bei dieser Methode wird die Probe mit einer periodischen Bewegung durch ein Gradiometer zweiter Ordnung ${ }^{4}$ gefahren. Das Gradiometer ist induktiv mit einer supraleitenden rf-Quanteninterferenzeinheit (rf-SQUID) verbunden. Das rf-SQUID nutzt den Josephson-Effekt und die Flussquantisierung aus, um die (durch die bewegte Probe) induzierte Änderung des magnetischen Flusses auf theoretisch ein Flussquant $\phi_{0} \sim 2.067 \cdot 10^{-15} \mathrm{~Wb}$ genau zu bestimmen. Unter der Betrachtung des typischen Probenvolumens in diesem System erhält man somit die Größenordnung der Standardabweichung mit $\sigma_{M} \sim 10^{-8}$ emu für eine Messung der Magnetisierung. Eine detaillierte Beschreibung der Funktionsweise des SQUIDs kann in einschlägiger Literatur [59, 60] nachgelesen werden. Die Probe kann in diesem MPMS System in einem Parameterraum von $T=4 \ldots 400 \mathrm{~K}$ und $H=0 \ldots 50 k O e$ vermessen werden.

\footnotetext{
${ }^{4}$ Supraleitende Pickup-Spule aus vier Windungen, bei der die zwei mittleren Wicklungen den beiden äußeren gegenüber entgegengesetzt verlaufen.
} 


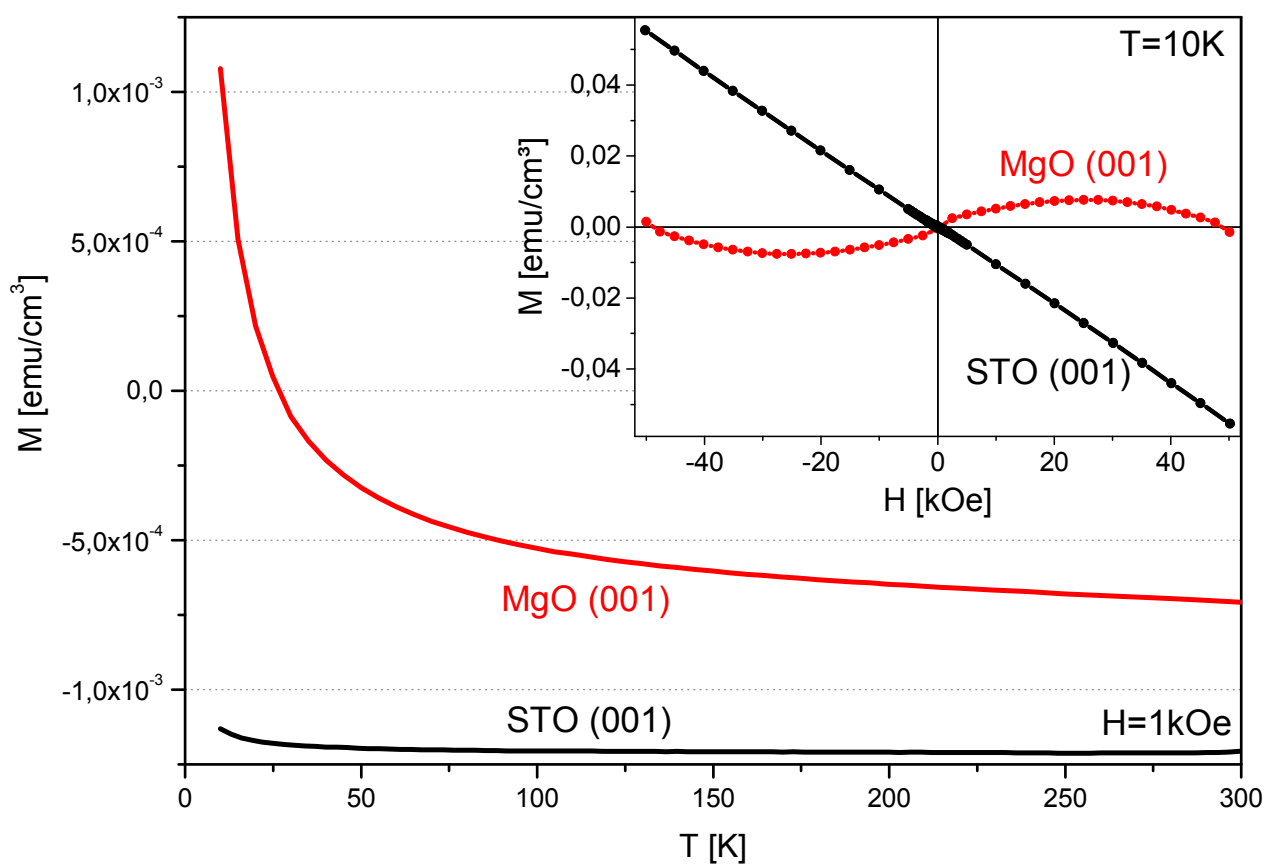

Abbildung 9: Magnetisierung als Funktion der Temperatur von einem $10 \cdot 5 \cdot 0.5 \mathrm{~mm}^{3}$ STO(001) (schwarz) und $\mathrm{MgO}(001)$ (rot) Substrat bei einem externen Feld von $1 \mathrm{kOe}$. Das eingebettete Diagramm zeigt die Magnetisierung der Substrate als Funktion des externen Feldes $H$ bei einer Temperatur von $10 K$.

Die Genauigkeit des MPMS Systems reicht theoretisch aus, dass der Fehler bei der Messung der Magnetisierung von typischen ferro/ferrimagnetischen dünnen Filmen kleiner ist als $0.1 \%$. Bei dieser Betrachtung wird jedoch davon ausgegangen, dass nur der perfekt installierte Film vermessen wird, was sich experimentell jedoch nicht bewerkstelligen lässt: Es ist möglich, dass der Film bezüglich der Bewegungsrichtung durch das Gradiometer um den Winkel $\phi$ leicht verkippt eingebaut ist. Da ferro/ferrimagnetische dünne Filme durch die magnetische Formanisotropie meist eine in-plane Anisotropie aufweisen [19], ist die Magnetisierung eines verkippten Filmes nicht parallel zum angelegten externen Feld. Dies hat zu Folge, dass der, in dem Gradiometer induzierte, Strom und damit die gemessene Magnetisierung um den Faktor $\cos (\phi)$ von dem wirklichen Wert abweicht ${ }^{5}$. Der Fluchtungsfehler $\phi$ beim Einbau der Proben kann auf einen Maximalwert von $\phi_{\max } \sim 15^{\circ}$ abgeschätzt werden, was zu einer Verminderung des Messsignals von $\sim-4 \%$ führt.

Zusätzlich lässt es sich nicht vermeiden, dass das Substrat und der Probenhalter bei der Messung beteiligt sind. Eine wichtige Größe für die Charakterisierung von ferri/ferromagnetischen

\footnotetext{
${ }^{5}$ Das Gradiometer ist supraleitend. Aus der zweiten London-Gleichung für Supraleiter [59] ist bekannt: $\operatorname{rot} \vec{j} \sim-\vec{B}$, mit dem durch die magnetische Flussdichte $B$ induzierten Supraleiterstrom $j$. Verwendung des Kelvin Stokes Theorems liefert $\int \vec{j} \cdot d \vec{s}=\int \vec{B} \cdot d \vec{A}$ mit der Flächenintegration über die Fläche $A$ und dem Linienintegral über die Kante der Fläche $s$. Daraus folgt $j \sim M \cdot A \cdot \cos (\phi)$, mit der Magnetisierung $M$.
} 
Materialien ist die Sättigungsmagnetisierung bei tiefen Temperaturen und hohen Feldern. Um diese Größe für dünne Filme zu bestimmen, muss die Magnetisierung des Hintergrundes, bestehend aus der Magnetisierung des Substrates und des Probenhalters, gewissenhaft abgezogen werden. Die temperaturabhängigen Magnetisierungmessungen für ein STO(001) und ein $\mathrm{MgO}(001)$ (zusammen mit dem Probenhalter) sind in Abb.9 dargestellt. Der Probenhalter ist eine diamagnetische Kunststoffkapsel. Das STO zeigt ein diamagnetisches Verhalten für alle untersuchten Temperaturen. Bei hohen Temperaturen zeigt das MgO Substrat auch ein diamagnetisches Verhalten, bei tiefen Temperaturen wird es allerdings klar paramagnetisch. Dieses Verhalten kann auf Verunreinigungen des MgO Kristalls zurück geführt werden. Für Filme auf STO Standard Substraten mit einem Volumen von $10 \cdot 5 \cdot 0.5 \mathrm{~mm}^{3}$ wurde eine Gerade mit dem Anstieg -2.8 $10^{-8}$ emu/Oe abgezogen, der sich aus dem Anstieg der magnetfeldabhängigen Magnetisierung des Substrates ergibt (siehe eingebettetes Diagramm in Abb.9). Auf einen para/diamagnetischen Hintergrund, wie er bei den MgO Substraten beobachtet werden kann, ist dieses Verfahren nicht übertragbar. Anhand des eingebetteten Diagramms in Abb.9 ist dieser Fakt leicht ersichtlich. In dieser Abbildung ist die magnetfeldabhängige Magnetisierung des MgO Substrates bei $10 K$ in rot dargestellt. Bei kleinen Feldern dominiert das paramagnetische Verhalten, sobald der Paramagnet gesättigt ist, dominiert einen diamagnetisches Verhalten. Für Filme auf $\mathrm{MgO}$ wurde zunächst die hier gezeigte Kurve abgezogen, anschließend war durch ein unterschiedlichen diamagnetischen Beitrag von Substrat zu Substrat, zumeist ein zusätzlicher linearer Hintergrund abzuziehen. Der Fehler der sich durch den Abzug des Hintergrundes ergibt lässt sich nur schwer abschätzen.

Bei der Bestimmung der Sättigungsmagnetisierung fließt auch das Volumen des Films ein. Dieses wurde über die Röntgenreflektometrie und über die Vermessung der beschichteten Oberfläche mit einem digitalen Messschieber bestimmt. Die Oberflächenvermessung ist mit einem Fehler von ca. 5\% behaftet. Insgesamt ergibt sich für die Magnetisierungsmessungen von dünnen Filmen ein systematischer Fehler von min. 5\%, wobei eher eine zu niedrige Magnetisierung gemessen wird.

Die Curie-Temperatur wird in dieser Arbeit aus der temperaturabhängigen Magnetisierungsmessung $M(T)$ über das Minimum der Funktion $M(T) \cdot d M(T) / d T \sim-1 /\left(T-T_{C}\right)(\operatorname{loga-}$ rithmische Ableitung) ermittelt ${ }^{6}$.

\footnotetext{
${ }^{6}$ An diesem Punkt weicht die Magnetisierung von dem Curie-Weiss-Verhalten, das bei hohen Temperaturen $T>T_{C}$ beobachtet werden kann, ab.
} 


\subsubsection{Magnetooptischer Kerr-Effekt}

Der magnetooptische Kerr-Effekt (MOKE) [61, 62] kann ausgenutzt werden um die magnetischen Eigenschaften eines dünnen Films qualitativ, schnell und ortsabhängig zu erfassen. Bei diesem Effekt wird die Polarisationsebene eines einfallenden Lichtstrahls bei der Reflexion an einer ferri/ferromagnetischen Oberfläche gedreht. Diese Drehung resultiert aus dem magnetischem zirkularen Dichroismus [63, 64]. Ähnlich wie in dem Ellipsometrie-Aufbau, beschrieben in Kap. 3.1.3, kann die Drehung des polarisierten Lichtes und damit die Magnetisierung über das PMSA-Prinzip unter Zuhilfenahme von Lock-in- und photoelastischer Modulations-Technik bestimmt werden [33, 34]. Ein solcher Aufbau, zusammen mit einem Helmholtz-Spulen-Paar, wurde benutzt, um die in dieser Arbeit angefertigten longitudinalen MOKE Messungen zu erstellen. Als Lichtquelle wurde dabei ein He-Ne-Laser benutzt. Da diese Methode nur eine Nebenrolle in dieser Arbeit spielt, wird hier nicht näher darauf eingegangen.

\subsubsection{Messung des elektrischen Widerstands}

Die Messungen des elektrischen Widerstands der dünnen Filme wurden in dem Physical Properties Measurement System (PPMS) der Firma Quantum Design oder der „Willi-Apparatur“ durchgeführt. Das PPMS ist ein kommerzielles System, das die Messung des DC-Widerstandes, mit Hilfe der Standard-Vierpunkt-Messbrücke der DC Option, in einem Parameterraum von $T=1.9-400 K$ und $H=0$ - 90kOe ermöglicht. Mit der „Willi-Apparatur" können ebenso unkompliziert und schnell temperaturabhängige Widerstandsmessungen durchgeführt werden. In dieser nicht-kommerziellen Anlage wird die Probe langsam in ein Heliumbad eingetaucht, wodurch der messbare Temperaturbereich mit $T=4.2-295 K$ festgelegt werden kann. Parallel wird der Widerstand über ein Keithley 2000 Multimeter und die Temperatur über eine thermische Diode ermittelt. Die Messungen beider Apparaturen haben gemein, dass der Widerstand über das Vierpunkt-Messverfahren (Kelvin-Messverfahren) ermittelt wurde. Dabei wird über die zwei äußeren Kontakte ein konstanter Strom angelegt, dessen Spannungsabfall über die beiden inneren Kontakte gemessen wird. Der Vorteil dieses Widerstand-Messverfahrens ist, dass dabei nicht die Kontakt- und Leitungswiderstände mitgemessen werden. Die Kontakte wurden jeweils mit Kupferdraht und Leitsilber präpariert. Alle in dieser Arbeit verwendeten Substrate zeigen einen sehr hohen Widerstand bei Raumtemperatur und ein isolierendes Verhalten. Folglich haben sie keinen Einfluss auf die Messungen des elektrischen Widerstands der dünnen Schichten. 


\section{A-Platz Ordnung in ferromagnetischen halbmetal- lischen Manganaten}

Bereits seit vielen Jahren erzeugen perowskitische Manganate mit der generellen Formel $\operatorname{Re}_{1-x}^{3+} A_{x}^{2+} M n_{1-x}^{3+} M n_{x}^{4+} O_{3}$, wobei $R e$ und $A$ jeweils Elemente aus der Gruppe der seltenen Erden $(L a, \operatorname{Pr}, N d, \ldots)$ bzw. der Erdalkalimetalle $(C a, S r, B a, \ldots)$ sind, ein reges wissenschaftliches Interesse. Verantwortlich dafür ist das Auftreten des CMR Effektes [2, 65], eine Änderung des elektrischen Widerstandes von mehreren Größenordnungen durch das Anlegen eines magnetischen Feldes sowie das halbmetallische ${ }^{7}$ Verhalten [66] dieser oxidischen Perowskite, die damit ein großes Potential für die Anwendung in der Spintronik besitzen.

Die Ausgangsverbindung für die Familie der CMR Manganate ist das $\mathrm{LaMnO}_{3}$ (LMO). Aufgrund der großen Coulomb-Abstoßung zwischen den 3d-Elektronen ist das LMO ein Mott-Hubbard Isolator [67]. Die $\mathrm{Mn}^{3+}$-Ionen liegen dabei in einer high-spin Elektronenkonfiguration vor. Die damit einhergehenden Jahn-Teller-Verzerrungen werden innerhalb des Kristalls zu einem kooperativen Effekt, d.h. die langen Achsen der Sauerstoffoktaeder zeigen dabei auf die kurzen Achsen, halb-gefüllte 3d-Orbitale zeigen auf leere 3d-Orbitale innerhalb einer Ebene. Zwischen diesen Ebenen stehen sich jeweils leere Orbitale gegenüber. Zusammen mit dem isolierenden Verhalten der $3 d$-Elektronen hat das zur Folge, dass ein A-Typ AFM in Bulk-Kristallen beobachtet wird [68], der über den Superaustausch erklärt werden kann: Innerhalb der beschriebenen Ebenen sind die 3d-Elektronen ferromagnetisch (FM) gekoppelt (2. GKA-Regel), während die Kopplung zwischen den Ebenen antiferromagnetisch (AFM) ist (1. GKA-Regel) [69].

Ersetzt man in LMO das $L a$ teilweise durch $A^{2+}$-Ionen dotiert man damit Löcher in das 3d-Band der $M n^{3+}$-Matrix ein, es entstehen $M n^{4+}$-Ionen. Dies hat zur Folge, dass eine Fülle von neuen Phasenübergängen in diesem Material beobachtet werden kann. In der Regel kann zum einen für $x<0.5 \mathrm{FM}$ in Verbindung mit einem metallischen Verhalten (FMM) und zum anderen für $x>0.5$ eine Ordnung der Orbitale und der Ladung in Verbindung mit AFM (AFM-COO) beobachtet werden [70]. Wie bereits in Kap.2.2 angesprochen, lässt sich der Metall/Isolator Übergang nahe $T_{C}$ und der FM für $L a_{1-x}^{3+} A_{x}^{2+} M n_{1-x}^{3+} M n_{x}^{4+} O_{3},(x<0.5)$ $[27,28]$ qualitativ durch den Doppelaustausch (DE) erklären. Für den $(x>0.5)$-Fall fin-

\footnotetext{
${ }^{7}$ Unter Halbmetallen versteht man in dieser Arbeit Materialien, die für die Elektronen mit unterschiedlichen Spin unterschiedliche elektrische Leitfähigkeiten zeigen. D.h. es existiert eine metallische Leitfähigkeit für Elektronen mit einer ausgezeichneten Spin-Richtung, während die Elektronen mit entgegengesetztem Spin ein isolierendes Verhalten aufweisen. Die hier verwendete Bezeichnung darf nicht mit Semimetall, Halbleiter oder der Bezeichnung Halbmetall (vergl. engl. metaloid) für eine Gruppe von Elementen aus dem Periodensystem zwischen den Metallen und den Nichtmetallen verwechselt werden.
} 


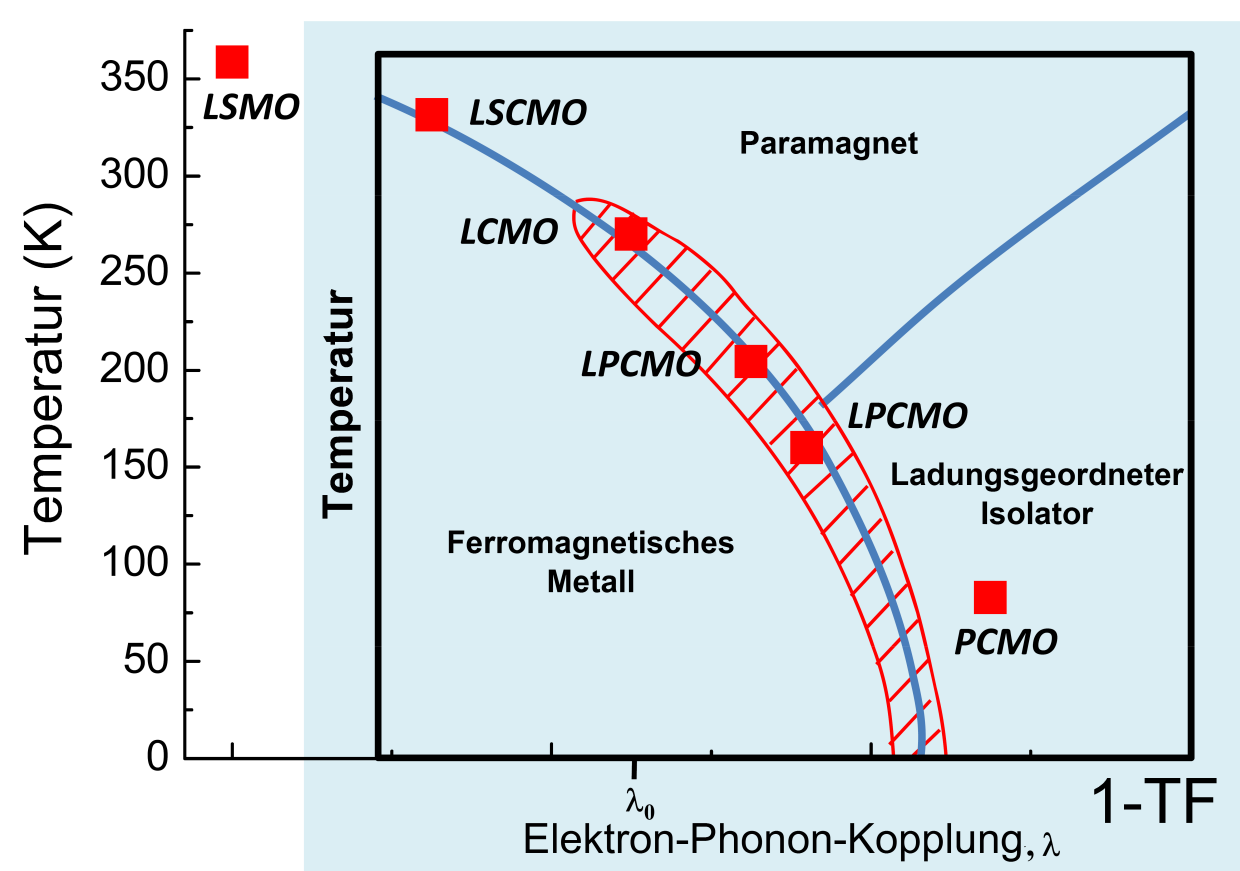

Abbildung 10: Generisches Phasendiagramm für CMR-Manganate aus [73], überlagert von einem experimentellen Phasendiagramm von optimal dotierten CMR Manganaten $(x=0.3)$ mit den Daten aus [74]. Bei diesem Diagramm handelt es sich um eine modifizierte Version aus der Literatur [75].

det der Superaustausch (SE) Anwendung. Mit dem Einbringen von $A^{2+}$-Ionen ändert man zusätzlich den chemischen Druck innerhalb des Kristalls durch die verschieden Ionenradien der Elemente, die auf dem A-Platz zu finden sind. Damit ändert man auch den $M n-O-M n$ Winkel und damit die Überlappung der Orbitale. Das hat wiederum einen direkten Einfluss darauf, wie stark die 3d-Elektronen gebunden bzw. delokalisiert sind. Somit wird auch die Austauschwechselwirkung zwischen den 3d-Elektronen, sowohl für den SE- (mit gebundenen Elektronen) als auch für den DE-Fall (mit delokalisierten Elektronen) durch den chemischen Druck beeinflusst. Der erstaunlich große Einfluss des Kristallgitter-Systems auf das Elektronensystem in Manganaten ist dabei auf eine große Elektron-Phonon-Kopplung $\lambda$, aufgrund des Jahn-Teller-Effektes zurückzuführen. Es wurde theoretisch gezeigt, dass $\lambda$ in Manganaten groß genug ist, dass die $e_{g}$-Elektronen bei Jahn-Teller-Verzerrungen lokalisiert sind. Das daraus resultierende Quasiteilchen (Elektron+Phonon über die Jahn-Teller-Verzerrung) bezeichnet man als Jahn-Teller-Polaron [71, 72].

Zusammenfassend können die Phasenübergänge in Manganaten durch die Lochkonzentration $x$ und durch die Gitterverzerrung, die im trivialsten Fall mit dem TF beschrieben werden kann, beeinflusst werden. Man spricht hierbei auch von der Kontrolle der Füllung (Parameter $x$ ) und der Bandbreite (Parameter TF) der elektronischen Bänder [67]. In dieser Arbeit wird weiter speziell der Fall $x \sim 0.33$ betrachtet, aus diesem Grund wird auf 
Variation von $x$ nicht weiter eingegangen. Bei der Variation von $T F$ unterscheidet man CMR-Manganate mit großer Bandbreite $0.98<T F<1$ (z.B. $\operatorname{La}_{0.67} \mathrm{Sr}_{0.33} \mathrm{MnO}_{3}$ ), mit mittlerer Bandbreite $0.96<T F<0.98$ (z.B. $\mathrm{La}_{0.67} \mathrm{Ca}_{0.33} \mathrm{MnO}_{3}$ ) und kleiner Bandbreite $T F<0.96$ (z.B. $\operatorname{Pr}_{0.67} \mathrm{Ca}_{0.33} \mathrm{MnO}_{3}$ ). Bemerkenswerterweise ist der Toleranzfaktor proportional zu der Elektron-Phonon-Kopplung $\lambda$ mit $\lambda \sim 1-T F$ [75]. Dieser Zusammenhang ist in Abb.10 dargestellt. Auf dem blauen Hintergrund ist das generalisierte Phasendiagramm für CMR-Manganate von Mathur und Littlewood [73] gezeigt. Es sind neben der paramagnetisch isolierenden Phase oberhalb der Übergangstemperaturen die zwei Hauptphasen der CMR-Manganate gezeigt: die FMM-Phase für kleine Werte von $\lambda$ und die AFM-COOPhase für große Werte von $\lambda$. Dabei ist der Phasenübergang für $\lambda<\lambda_{0}$ ein kontinuierlicher Phasenübergang zweiter Ordnung, der für $\lambda>\lambda_{0}$ in einen Phasenübergang erster Ordnung übergeht. Letzterer führt zu einer elektronischen Phasenseparation (PS), die durch den schraffierten Bereich dargestellt werden soll. Die PS steht in direktem Zusammenhang mit dem in diesem Bereich auftretenden hohen CMR [75, 76]. Auf diesem generellen Phasendiagramm sind die experimentellen Werte von Hwang et al. [74] für optimal dotierte Manganate $\left(\mathrm{La}_{0.7} \mathrm{Sr}_{0.3} \mathrm{MnO}_{3}\right.$ - LSMO, $\mathrm{La}_{0.7}\left(\mathrm{Sr}_{1-z} \mathrm{Ca} a_{z}\right)_{0.3} \mathrm{MnO}_{3}$ - LSCMO, $\mathrm{La}_{0.7} \mathrm{Ca}_{0.3} \mathrm{MnO}_{3}$ - LCMO, $\left(\mathrm{La}_{1-y} \mathrm{Pr}_{y}\right)_{0.7} \mathrm{Ca}_{0.3} \mathrm{MnO}_{3}$ - LPCMO, $\mathrm{Pr}_{0.7} \mathrm{Ca}_{0.3} \mathrm{MnO}_{3}$ - PCMO ) als Funktion von $1-\mathrm{TF}$ dargestellt. Die Übereinstimmung der beiden Diagramme kann damit verstanden werden, dass mit der Abnahme von TF, die orthorhombische Verzerrung größer wird und damit die Bandbreite abnimmt. Somit nimmt $T_{C}$ proportional zu $T F$ ab und schließlich sind die Elektronen stärker an das Gitter gebunden, es entsteht eine AFM-COO-Phase [74].

Es gibt zwei signifikante Punkte in diesem Phasendiagramm, die für spintronische Anwendungen interessant sind: Zum einen ist das der große CMR rund um das LPCMO System $\left(\lambda<\lambda_{0}\right)$, zum anderen das LSMO als ferromagnetisches Halbmetall bei hohen Temperaturen $(T F \sim 1)$. In den nächsten zwei Kapiteln wird der Einfluss von der A-Platz Ordnung auf diese zwei Systeme untersucht. Dabei wurde die elektronische PS künstlich in teilgeordneten $\left(\mathrm{La}_{0.5} \mathrm{Pr}_{0.5}\right)_{0.67} \mathrm{Ca}_{0.33} \mathrm{O}_{3}$ modelliert. In LSMO wurde hingegen untersucht, ob es möglich ist, die Curie-Temperatur mit der Hilfe von künstlicher A-Platz Ordnung zu erhöhen. 



\subsection{Künstliche elektronische Phasenseparation in teilgeordneten $\left(\mathrm{La}_{0.5} \mathrm{Pr}_{0.5}\right)_{0.67} \mathrm{Ca}_{0.33} \mathrm{MnO}_{3}$}

\subsubsection{Elektronischen Phasenseparation und Kolossaler Magnetowiderstand in $\left(\mathrm{La}_{1-\mathrm{y}} \mathrm{Pr}_{\mathrm{y}}\right)_{0.67} \mathrm{Ca}_{0.33} \mathrm{MnO}_{3}$}

Vor 15 Jahren wurde bereits gezeigt, dass in dem $\left(\mathrm{La}_{1-y} \mathrm{Pr}_{y}\right)_{0.67} \mathrm{Ca}_{0.33} \mathrm{MnO}_{3}$ (LPCMO) System eine elektronische PS nahe $T_{C}$ stattfindet. Das bei hohen Temperaturen paramagnetische Material bildet dabei sowohl FMM als auch AFM-COO Bereiche unter Kühlung/Anlegen eines externen Feldes aus. Mit Hilfe dieser PS konnte auch ein Ansatz für die Erklärung des großen CMR erstellt werden [76]. Die genauen Wechselwirkungen zwischen diesen konkurrierenden Phasen sind damit jedoch nicht vollständig verstanden.

In Abb.11 ist der spezifische Widerstand (a) und die Magnetisierung (b) eines dünnen $\left(\mathrm{La}_{0.6} \mathrm{Pr}_{0.4}\right)_{0.67} \mathrm{Ca}_{0.33} \mathrm{MnO}_{3}$ Filmes auf $\mathrm{MgO}(100)$ Substrat als Funktion der Temperatur $T$ und des magnetischen Feldes $H$ dargestellt (Daten aus [77, 78]). Der Metall/Isolator Übergang (MIT) bei der Temperatur $T_{M I} \sim T_{C} \sim 190 K$ ist klar zu erkennen. In einem relativ engen Intervall um diese Temperatur ist ein hoher $\mathrm{CMR}=(R(0)-R(H)) / R(H)$ vorhanden. Der Phasenübergang erster Ordnung ist klar durch die Existenz einer Hysterese zwischen den Aufwärm/Abkühl- bzw. Feldauf/Feldab-Kurven (in Abb.11 durch Pfeile angedeutet) zu erkennen. Dabei muss für die feldabhängige Magnetisierung bei $T_{C} \sim 190 \mathrm{~K}$ beachtet werden, dass neben der ferromagnetische Hysterese durch Domänenwachstum/drehung bei niedrigeren Feldern $H<0.3 k O e$, eine zusätzliche Hysterese zwischen $0.3 k O e<H<$ $8 k O e$ vorhanden ist. Der feldabhängige Widerstand bei $T_{M I} \sim T_{C} \sim 190 \mathrm{~K}$ zeigt für die Feldauf-Kurve ein Maximum im Widerstand nahe dem Koerzitivfeld (in Abb.11 angedeutet durch die blauen Markierungen). Dieses Verhalten ist ähnlich dem Widerstandsverlauf, der in Tunnel-Magnetowiderstands-Experimenten beobachtet werden kann [19]. Das Koerzitivfeld (geschlossene Symbole in Abb.12) zeigt eine wurzelförmige Temperaturabhängigkeit für $T<<T_{C}$ und steigt nahe $T_{C}$ stark an, bevor es für $T>T_{C}$ verschwindet. In breitbandigen Manganaten (LSMO, LCMO) wird im Kontrast dazu eine lineare Temperaturabhängigkeit von $H_{C}$ beobachtet und der Peak nahe $T_{C}$ taucht nicht auf [78, 79]. Das wurzelförmige Verhalten in LPCMO ist dabei zurückzuführen auf die Anwesenheit von eindomänigen, ferromagnetischen Partikeln [80] mit einer superparamagnetischen Block Temperatur von $T_{B} \sim 1.05 \cdot T_{C}$. Die Domänengröße kann damit auf $\delta \sim 6-8 n m$ abgeschätzt werden [78]. Das Maximum von $H_{C}$ nahe $T_{C}$ ist dabei ein klares Indiz, dass in diesem phasenseparierten System eine Austauschwechselwirkung zwischen FM- und AFM-Domänen statt findet (wie bereits in anderen AFM/FM gekoppelten Systemen beobachtet [81-83]). Aus den anharmo- 
4 A-Platz Ordnung in ferromagnetischen halbmetallischen Manganaten
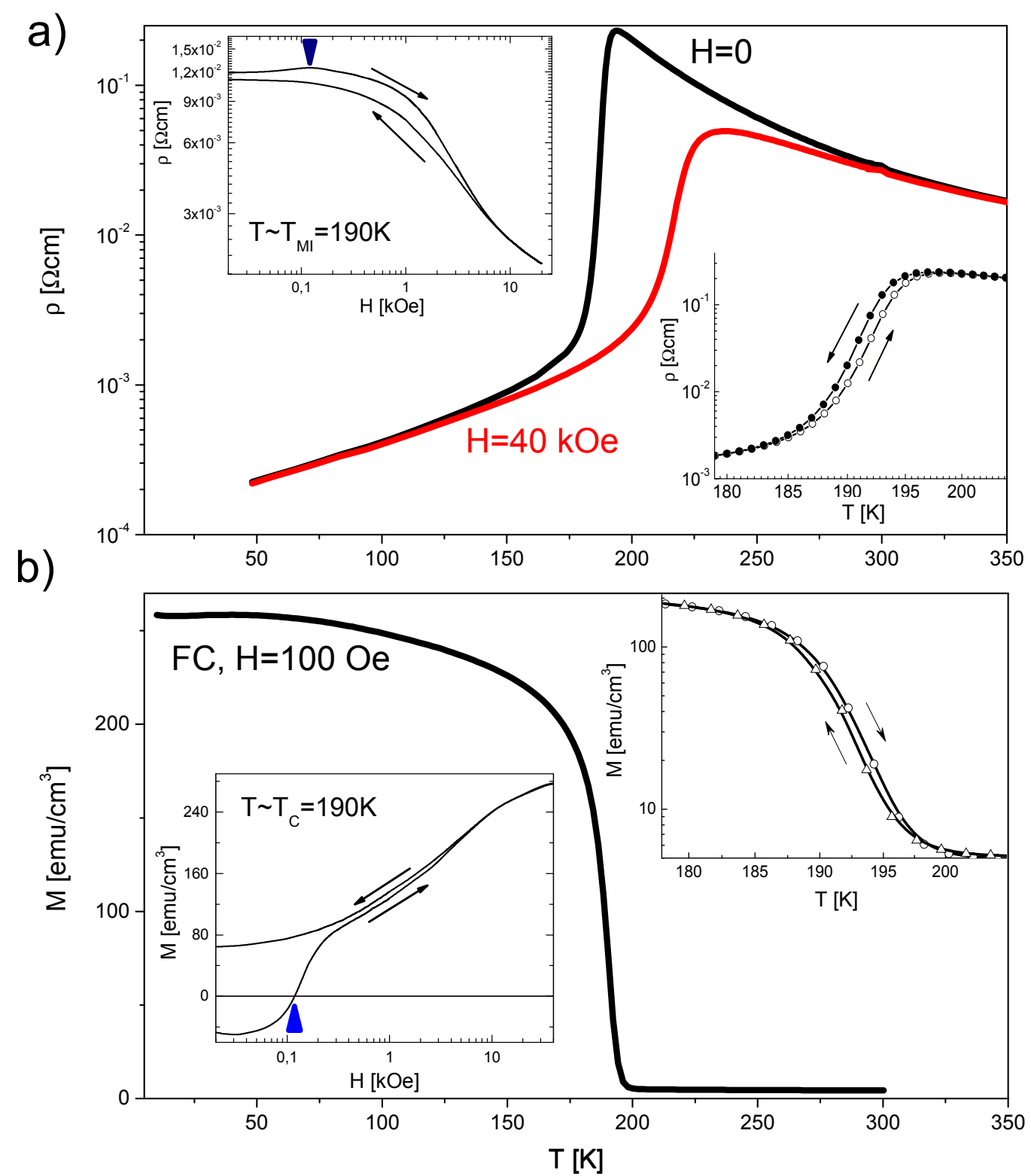

Abbildung 11: a) Spezifischer Widerstand $\rho$ als Funktion der Temperatur $T$ eines $\left(\mathrm{La}_{0.6} \mathrm{Pr}_{0.4}\right)_{0.67} \mathrm{Ca}_{0.33} \mathrm{MnO}_{3}$ Filmes auf $\mathrm{MgO}(100)$ Substrat bei einem externen magnetischen Feld von 0Oe (schwarz) und 40kOe (rot). Es ist sowohl die Abkühl- als auch die Aufwärmkurve gezeigt. Das rechte, eingebettete Diagramm zeigt diese Funktion vergrößert, zur Verdeutlichung der thermalen Hysterese. Das linke, eingebettete Diagramm zeigt die Feldabhängigkeit des spezifischen Widerstands bei einer Temperatur nahe $T_{C}$ bei $190 \mathrm{~K}$. b) Die Magnetisierung der gleichen Probe bei einem externen Feld von 100Oe. Die eingebetteten Diagramme sind wie in a) zu begreifen. Die Pfeile zeigen die Messrichtung an. Die blauen Markierungen in den feldabhängigen Kurven deuten an, dass ein Maximum im Widerstand für Temperaturen nahe $T_{C}$ bei dem Koerzitivfeld zu finden ist. Die Daten sind aus [77, 78] entnommen. 


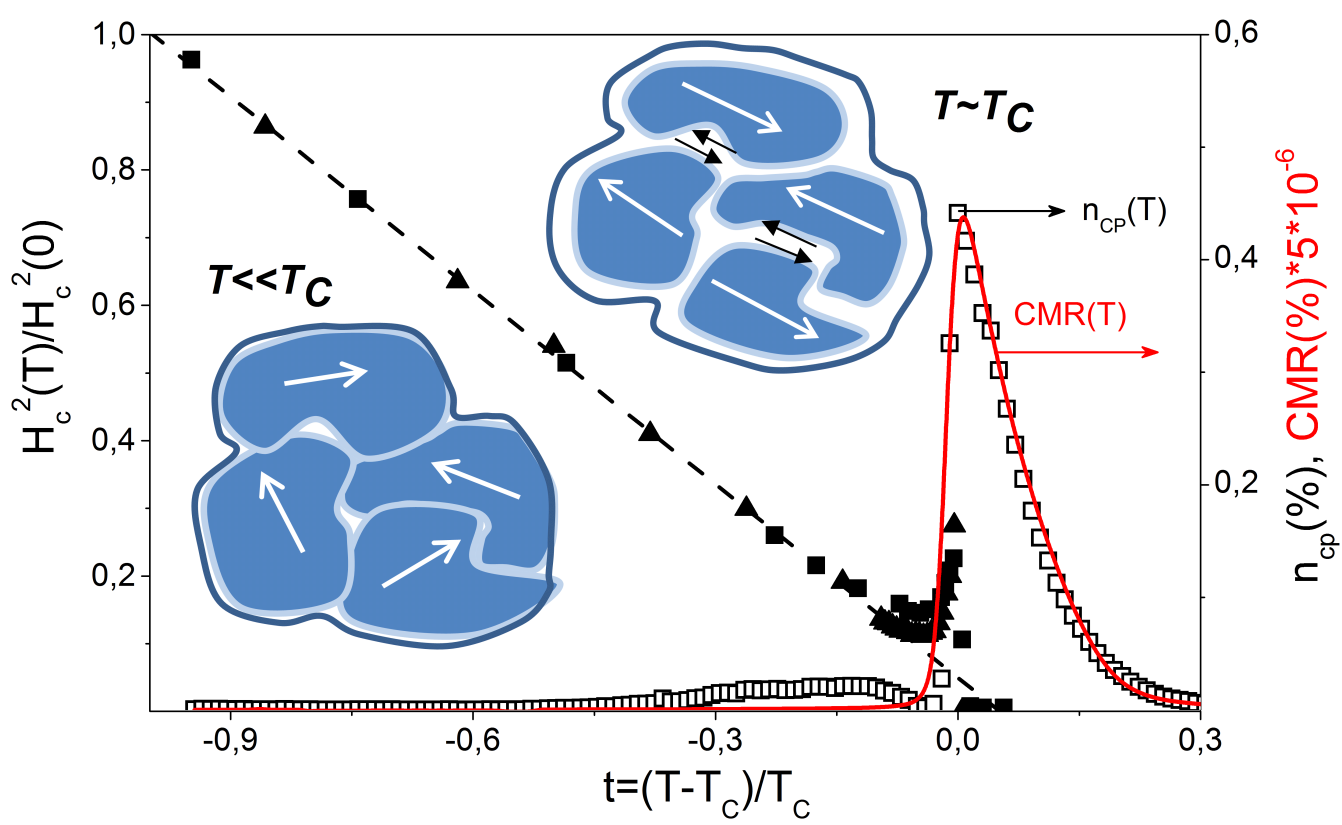

Abbildung 12: Die geschlossenen Symbole zeigen das Quadrat des normierten Koerzitivfeldes $h_{c}=$ $\left(H_{c}(T) / H_{c}(0)\right)^{2}$ als Funktion der normierten Temperatur $t=\left(t-T_{C}\right) / T_{C}$, für LPCMO Filme mit $y=0.4$ (Quadrate) und $y=0.375$ (Dreiecke). Die gestrichelte Linie zeigt die lineare Anpassung mit der eine Block Temperatur von $T_{B}=1.05 \cdot T_{C}$ bestimmt werden kann. Die offenen Symbole zeigen die relative Anzahl der korrelierten Polaronen $n_{C P}(T)$. Mit dieser Anzahl skaliert die CMR(T) Kurve (rot). Die Skizzen zeigen das Modell der magnetischen Struktur in der Nähe und weit weg von $T_{C}$. Die Daten sind aus [78] entnommen.

nischen Beträgen aus AC-Widerstandsmessungen (spezifischer: der dritten Harmonischen) kann die relative Anzahl der korrelierten Jahn-Teller-Polaronen $n_{C P}$ temperaturabhängig bestimmt werden (offene Symbole in Abb.12). Diese Größe hat mit 0.5\% ein Maximum bei $T \sim T_{C}$ und skaliert mit der $\operatorname{CMR}(T)$ Kurve [79]. Benutzt man die Korrelationslänge der Jahn-Teller-Polaronen von $\delta \sim 1$ - 2nm, die aus Neutronen-Streuexperimenten gewonnen wurde [84, 85] und legt die daraus resultierende AFM-COO Phase auf die Domänengrenzen, um die ferromagnetischen eindomänigen Partikel herum, kann man aus der Anzahl $n_{C P}$ einen Durchmesser von $\delta \sim 9 n m$ für die FM-Domänen erhalten [78]. Dieses Ergebnis stimmt sehr gut mit dem Resultat für $\delta$ aus der Abschätzung über die Block Temperatur $T_{B}$ überein. Aus diesem Bild erhält man ein Modell für die PS in LPCMO (skizziert in Abb.12), welches die beschriebenen Ergebnisse hinreichend erklären kann: Nahe $T_{C}$ ist eine AFM-COO Phase aus korrelierten Jahn-Teller-Polaronen zwischen den FM Partikeln vorhanden, die zu einer AFM Kopplung zwischen den FM-Partikeln führt. Legt man ein kleines magnetisches Feld an, erwartet man in diesem Modell zunächst das beobachtete TMR-artige Verhalten: Für $H=H_{C}$ ist der Widerstand maximal, aufgrund der hohen AFM-Ordnung zwischen den FM Partikel. Erhöht man weiter das externe Feld auf $H>H_{C}$ richten sich die FM-Domänen parallel 
4 A-Platz Ordnung in ferromagnetischen halbmetallischen Manganaten

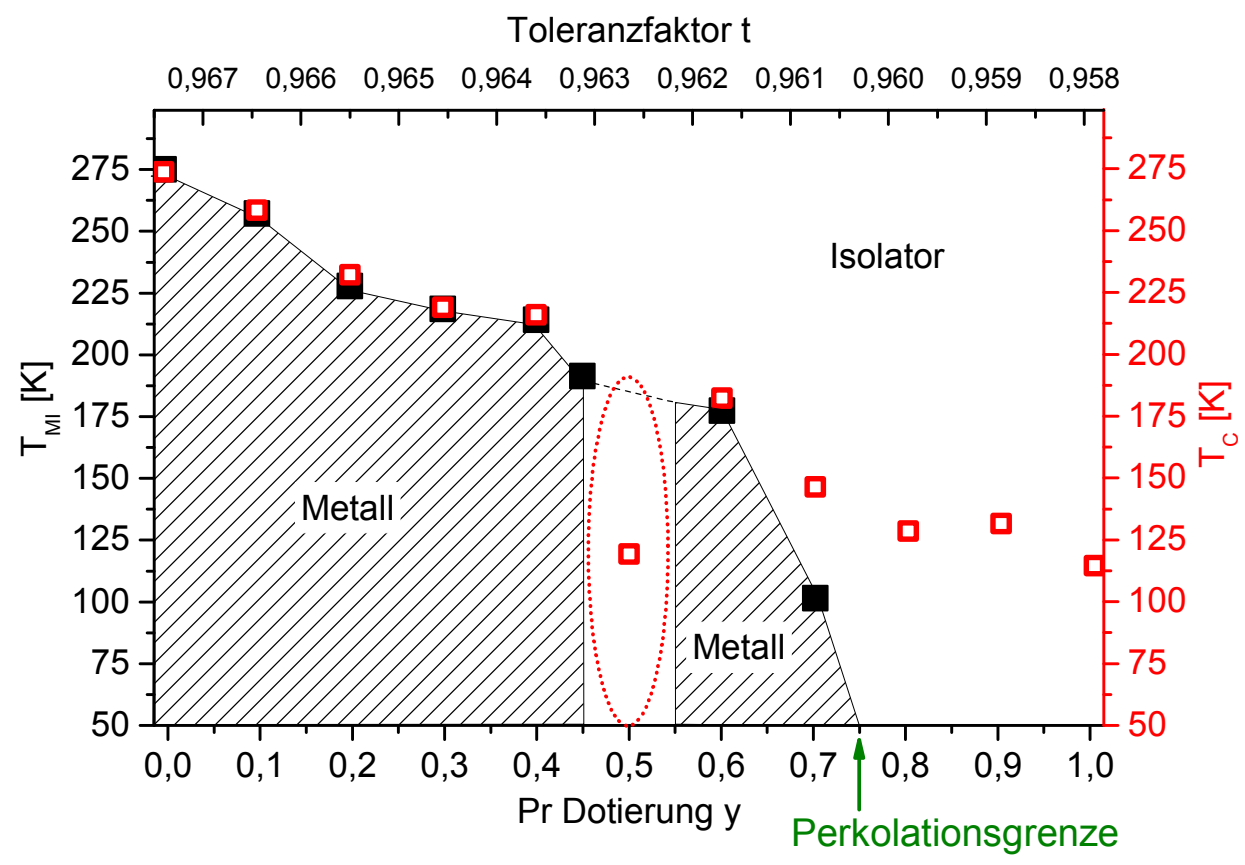

Abbildung 13: Die MIT Temperatur $T_{M I}$ (schwarz) und die Curie-Temperatur $T_{C}$ (rot) als Funktion der Pr-Dotierung y von $\left(\mathrm{La}_{1-y} \mathrm{Pr}_{y}\right)_{0.67} \mathrm{Ca}_{0.33} \mathrm{MnO}_{3}$ Filmen auf $\mathrm{MgO}(100)$ Substrat. Ab der Perkolationsgrenze von $y_{c} \sim 0.75$ ist kein MIT mehr im Material vorhanden. Es zeigt sich eine Unstetigkeit bei $y=0.5$ bei der, im Gegensatz zu bekannten Daten in Bulk-Polykristallen [74, 76], kein MIT und ein stark verringertes $T_{C}$ gemessen wird. Die Daten sind aus [77] entnommen.

aus. Durch den halbmetallischen Charakter des Transports, fällt der Widerstand in diesem Bereich drastisch ab (CMR). Die Hysteresen im Widerstand und in der Magnetisierung schließen sich für mehrere $k O e$. Bei diesen hohen Feldern werden die antiferromagnetischen Korrelationen zerstört. Senkt man die Temperatur in diesem Modell auf $T<<T_{C}$ nimmt die Anzahl der korrelierten Polaronen stark ab. Damit existiert keine AFM-Kopplung zwischen den FM Partikeln. In diesem Bereich des Phasendiagrammes ist damit auch kein CMR zu finden. Die Daten zeigen klar, dass die Größe des CMR in einem direkten Zusammenhang mit der PS und der Anwesenheit von korrelierten Polaronen steht [78].

Mit der Variation des $L a / P r$ Verhältnisses im LPCMO System mit $x \sim 0.33$ bewegt sich das Manganat zwischen einer mittleren Bandbreite (LCMO) und einer kleinen Bandbreite (PCMO). Das LCMO bildet bei niedrigen Temperaturen eine FMM-Phase mit einem Phasenübergang nahe der 2.Ordnung und einen geringen Anteil an korrelierten Polaronen aus $[75,86]$. Bei PCMO wird diskutiert, dass sich bei tiefen Temperaturen ein COOIsolator mit einem gekanteten AFM- [87] oder ein elektronisch phasenseparierter Zustand, der hauptsächlich aus einer isolierenden AFM-COO und einem kleinen Anteil einer FMM Phase [88, 89] besteht, ausbildet. Für das LPCMO System bedeutet das folglich, dass mit der Erhöhung des Pr-Anteils y der Anteil der COO-Phase stetig ansteigt und sich der MIT 
zu tieferen Temperaturen verschiebt. Dies spiegelt sich in den experimentellen Ergebnissen wieder $[74,76,77]$ (siehe Abb.13). Es existiert ein kritischer Wert $y_{c}$, so dass für $y>y_{c}$ kein MIT stattfindet. Dieser Wert wird auch Perkolationsgrenze genannt: Der Anteil der FMM-Phase ist dabei nicht mehr groß genug, damit sich ein perkolativer Pfad durch das Material ausbilden kann, über den der elektrische Transport vermittelt wird [76]. Somit bleibt das Material über den gesamten Temperaturverlauf isolierend. Für dünne LPCMO Filme auf MgO-Substrat (siehe Abb.13) ist die Perkolationsgrenze bei $y_{c} \sim 0.75$ zu finden [77]. Dennoch kann in dem Bereich $y>y_{c}$ ein MIT mit dem Anlegen eines externen Feldes induziert werden [77]. Dabei schmilzt man die COO-Phase (zum Teil) mit dem externen Feld auf $[78,90]$ und verschiebt damit die Volumenanteile der AFM-COO- und FMM-Phase. In dem in Abb.13 aufgeführten Diagramm für dünne LPCMO Filme gibt es einen kritischen Punkt bei $y=0.5$ (in Abb.13 mit einer roten Ellipse markiert). Die Magnetisierung ist in Größe und Übergangstemperatur verringert, es existiert kein MIT ohne externes Feld [77]. Diese Unstetigkeit wurde in Bulk-Polykristallen nicht registriert $[74,76]$ (in Abb.13 angedeutet durch die schwarze gestrichelte Linie). Generell zeigen die Proben mit $y=0.5$ ähnliche magnetische und elektrische Eigenschaften wie LPCMO mit $y \sim 0.9$ [77]. Bislang existiert keine hinreichende Erklärung für diese Unstetigkeit. Eventuell existiert in diesem Teil des Phasendiagrammes eine gewisse Tendenz zur Ordnung oder zur Unordnung der drei verschiedenen A-Platz Ionen, deren Anzahl hier etwa gleich groß ist (nominell $L a_{\sim 0.33} \operatorname{Pr}_{\sim 0.33} \mathrm{Ca}_{\sim 0.33} \mathrm{MnO}_{3}$ ). Eine künstliche Ordnung der A-Platz Ionen durch die Präparation von $\left(\mathrm{La}_{0.67} \mathrm{Ca}_{0.33} \mathrm{MnO}_{3}\right) /\left(\mathrm{Pr}_{0.67} \mathrm{Ca}_{0.33} \mathrm{MnO}_{3}\right)$ (LCMO/PCMO) Überstrukturen kann einerseits Einblicke in diese Thematik bringen, andererseits stellen diese Überstrukturen ein künstlich, lagenweise PS System (in FMM-LCMO und CAFM-COOPCMO Lagen) dar, welches sich geometrisch klar von der natürlichen PS unterscheidet. Daraus können neue Erkenntnisse über die Wechselwirkungen und die kritischen Größen zwischen bzw. in den zwei konkurrierenden Phasen gewonnen werden.

\subsubsection{Präparation von $\left(\mathrm{La}_{0.67} \mathrm{Ca}_{0.33} \mathrm{MnO}_{3}\right) /\left(\mathrm{Pr}_{0.67} \mathrm{Ca}_{0.33} \mathrm{MnO}_{3}\right)$ Überstrukturen}

Das $\mathrm{La}_{0.67} \mathrm{Ca}_{0.33} \mathrm{MnO}_{3}$ (LCMO) System wurde zunächst, wie bereits in einer früheren Arbeit beschrieben [77], eingestellt: Dazu wurde das $L a / M n$-Verhältnis mit der Präparation von $\mathrm{LaMnO}_{3}$ Filmen auf $\mathrm{MgO}(001)$, näherungsweise ermittelt, indem die Oberflächenmorphologie schrittweise optimiert wurde. Das $\mathrm{La} / \mathrm{Ca}$-Verhältnis wurde anschließend ermittelt, indem die Curie- bzw. die MIT-Temperatur $T_{C}$ bzw. $T_{M I}$ sowie die Schärfe dieses Phasenüberganges, ausgedrückt durch den Wert $\alpha_{R}=\operatorname{Max}((T / R) \cdot(d R / d T))$, optimiert wurde. Im LCMO System ist das maximale $T_{C} \sim 275^{\circ} \mathrm{C}$ gerade bei $x=0.33$ anzutreffen [91]. Der absolu- 
4 A-Platz Ordnung in ferromagnetischen halbmetallischen Manganaten

te stöchiometrische Fehler des $L a / C a$-Verhältnisses im Film $\delta x$ kann, aufgrund der Breite des Maximums von $T_{C}$ in dem Bulk-Kristall-Phasendiagramm mit $\delta x \sim 0.03$ abgeschätzt werden. Die ermittelten Koeffizienten für das $L a / M n$ - und das $L a / C a$-Verhältnis unterscheiden sich nicht von den Koeffizienten aus der Literatur [77]. Diese Koeffizienten wurden auch für das $\operatorname{Pr}_{0.67} \mathrm{Ca}_{0.33} \mathrm{MnO}_{3}$ (PCMO) System angenommen $(\mathrm{Pr} / \mathrm{Mn}=\mathrm{La} / \mathrm{Mn}$ und $\operatorname{Pr} / C a=L a / C a)$.

Für die Präparation dieses Systems wurde das $\mathrm{MgO}(001)$ als Substrat gewählt. Der Unterschied zwischen den pseudokubischen Gitterkonstanten von $\mathrm{MgO}$ (mit $\sim 0.42 \mathrm{~nm}$ ) und LPCMO (mit $\sim 0.386 n m$ ) ist mit $\sim 8 \%$ sehr groß. Diese große Diskrepanz führt bei dem Wachstum der allerersten Lagen des Manganatmaterials zu einer vermehrten Ausbildung von Versetzungen. Nach wenigen Lagen $\left(\sim 3 u . c .^{8}\right)$ wächst das Material epitaktisch und unverspannt auf dem Substrat auf. Dieser Mechanismus funktioniert besonders gut für bestimmte Größenverhältnisse der Gitterkonstanten und Gitterstruktur von Substrat und Filmmaterial $^{9}$ und wird "domain-matching-epitaxy" genannt [92]. In dem LPCMO System, in dem der chemische Druck über die Dotierung variiert wird und diese somit einen großen Einfluss auf die physikalischen Eigenschaften des Systems hat, ist es von Vorteil, wenn der Film relaxiert wächst und keine weiteren Verspannungen über das Substrat in das System eingebracht werden. Die Abbildungen 14 a) und b) zeigen das epitaktische Wachstum von LCMO und PCMO anhand von XRD-Messungen. Diese Messungen offenbaren keine weiteren Reflexe neben den Film(001)- und den Substrat-Reflexen (das Spektrum des blanken Substrates ist in Abb.14 in rot gezeigt). Dabei ist anzumerken, dass der hier gezeigte $\sim 100 \mathrm{~nm}$ dicke PCMO Film auf einem $\sim 8 n m$ dicken LCMO Puffer-Film gewachsen wurde. Dies ist nötig, da PCMO polykristallin-texturiert in (001) und (011)-Richtung direkt auf MgO aufwächst [77], jedoch nicht auf LCMO, wie in Abb.14b gezeigt wird. Die out-of-plane Gitterkonstante von LCMO liegt bei $~ 0.3863 \mathrm{~nm}$, nahe der Gitterkonstante von Bulk-LCMO mit 0.3867nm [93]. Das PCMO scheint hingegen teilweise verspannt auf LCMO aufzuwachsen: zum einen sind die (001) Reflexe verbreitert, zum anderen ist die out-of-plane Gitterkonstante mit 0.384nm kleiner als die von Bulk-PCMO mit 0.385nm [94]. Die glatte Oberflächenflächenmorphologie des $\sim 80 \mathrm{~nm}$ dicken LCMO und des $\sim 100 \mathrm{~nm}$ PCMO Films mit dem quadratischen Mittel der Rauigkeit $0.43 \mathrm{~nm}$ bzw. $0.71 \mathrm{~nm}$, gezeigt durch die STM-Aufnahmen in Abb.14, zeugen von der guten kristallinen Qualität der Filme.

Die Magnetisierung für LCMO bestätigt, wie bereits erwähnt, eine Curie-Temperatur von $T_{C}=275 K$ und eine Sättigungsmagnetisierung von $M_{s} \sim 3.6 \mu_{B} / M n$ bei tiefen Temperaturen (siehe Abb.15). Dieser Wert entspricht näherungsweise der maximalen, theoretischen

\footnotetext{
${ }^{8}$ u.c. ist die Größe einer pseudokubischen Gitterkonstante des Materials.

${ }^{9} \mathrm{Im}$ Fall von LCMO/MgO ist dieses Verhältnis 11/12.
} 
4.1 Künstliche elektronische Phasenseparation in teilgeordneten LPCMO

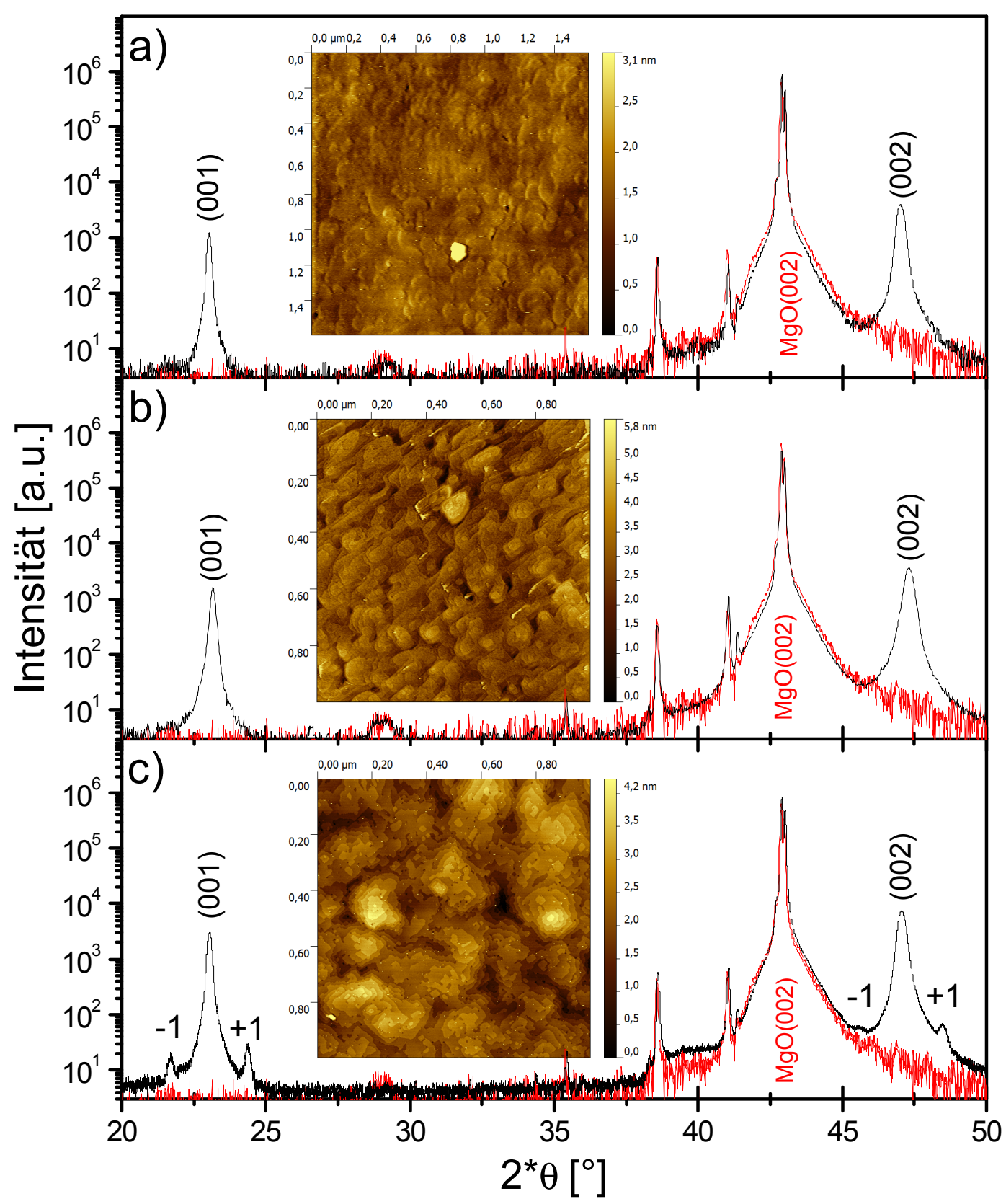

Abbildung 14: XRD-Spektren von a)LCMO, b)PCMO auf LCMO Puffer-Film, und

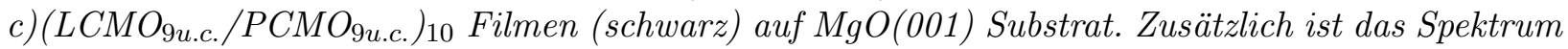
des Substrates in rot gezeigt. In allen Spektren sind keine weiteren Reflexe neben den Film(00l)-, Substrat- und den Überstruktur-Reflexen in c) (markiert mit -1 und +1 ) zu erkennen. Die eingebetteten Bilder zeigen die STM-Aufnahmen der entsprechenden Proben. 
4 A-Platz Ordnung in ferromagnetischen halbmetallischen Manganaten

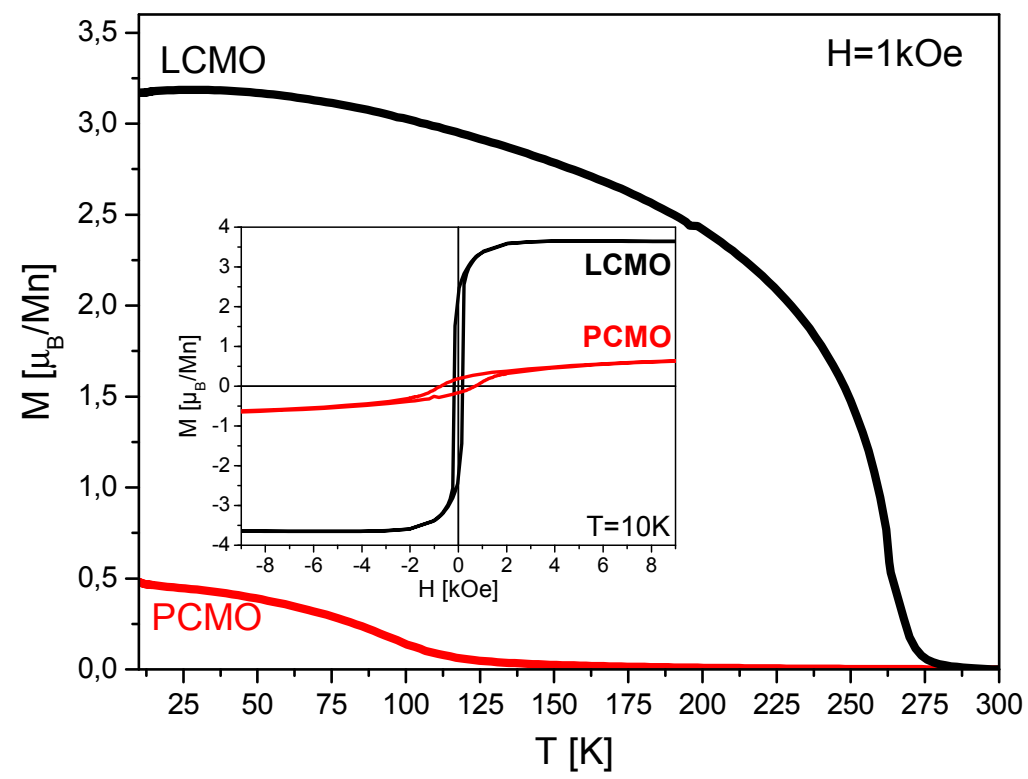

Abbildung 15: Magnetische Eigenschaften von LCMO und PCMO auf $M g O(001)$ $M(T)$-Kurven bei einem externen Feld von $1 k O e$ von einem 80 $\mathrm{nm}$ LCMO (schwarz) und einem 100nm PCMO Film auf einem 8nm LCMO Puffer-Film (rot) auf MgO(001)

Substrat. Das eingebettete Diagramm zeigt die $M(H)$ Hysterese-Schleifen der erwähnten Proben nach dem Einkühlen im Nullfeld auf eine Temperatur von $10 K$.

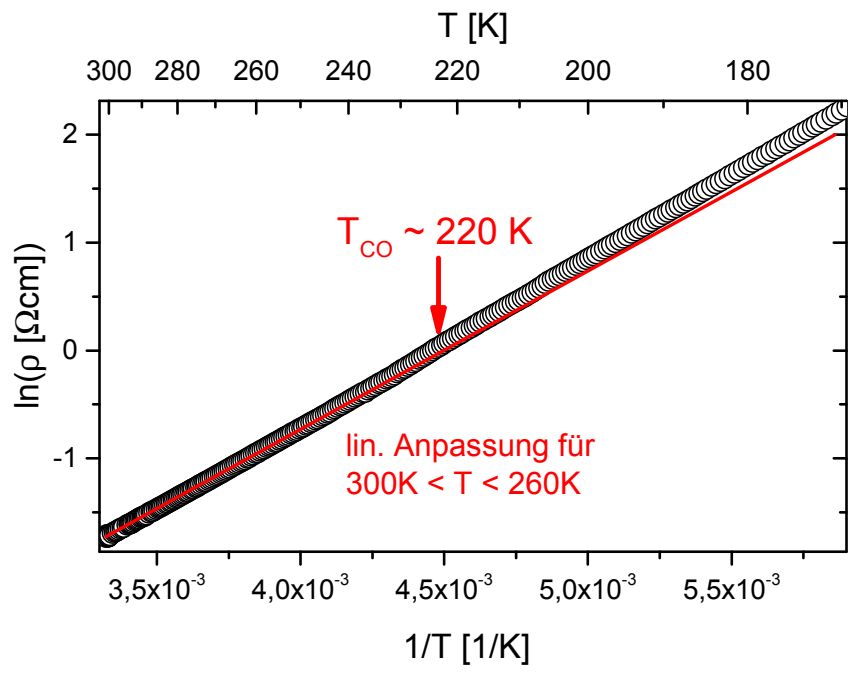

Abbildung 16: Natürlicher Logarithmus des spezifischen elektrischen Widerstandes $\ln (\rho)$ als Funktion der reziproken Temperatur $1 / T$ für einen 80nm PCMO-Film auf MgO(001)-Substrat. Die rote Gerade ist eine lineare Anpassung für die Daten zwischen $300-260 K$. Diese Gerade weicht für tiefere Temperaturen ab einer Temperatur von $220-230 K$ stark von den Messdaten ab. Nach Yang et al. ist an diesem Punkt die Ladungsordnungs-Temperatur $T_{C O}$ zu finden [95]. 
4.1 Künstliche elektronische Phasenseparation in teilgeordneten LPCMO

Sättigungsmagnetisierung, die man für diese $e_{g}$-Elektronendotierung erwartet: $M_{s, \text { theo }}=0.67 \cdot 4 \mu_{B} / M n^{3+}+0.33 \cdot 3 \mu_{B} / M n^{4+}=3.67 \mu_{B} / M n$. Dieser Film zeigt nahe $T_{C}$ einen MIT und in diesem Phasenübergangsbereich einen CMR (mit der Definition $\operatorname{CMR}(H)=$ $(R(0)-R(H)) / R(H))$ von $\operatorname{CMR}(70 k O e) \sim 300 \%$. Der PCMO Film mit den adaptierten Koeffizienten aus dem LCMO System ist über den gesamten Temperaturbereich isolierend, zusätzlich existiert eine erhöhte Magnetisierung unterhalb einer Temperatur von $110 K$ (siehe Abb.15). Dieses Verhalten wurde bereits beobachtet: In PCMO Bulk-Kristallen (mit $x=0.33$ ) liegt bei tiefen Temperaturen $\leq 100 K$ ein gekanteter AFM [87] oder eine FM/AFM-PS [88, 89] vor. Die PCMO $M(H)$ Hysterese-Schleife bei $10 K$ zeigt eine kleine Remanenz und damit ein kleines ferromagnetisches Moment. Dieses ferromagnetische Moment könnte auf den 8nm LCMO Puffer-Film und/oder auf FMM-Bereiche, die sich bei einer PS bilden, zurückzuführen sein. Das angelegte maximale Feld in dieser Messung von $50 k O e$ und in der feldabhängigen Widerstandsmessung von 70kOe [77] reicht nicht aus, um einen metamagnetischen Übergang in eine metastabile FMM Phase zu erzwingen, wie er in PCMO-Bulk-Kristallen beobachtet wird [96]. Man spricht in diesem Zusammenhang auch von dem Aufschmelzen der korrelierten Polaronen [97], ein Prozess der in dem, für diese Arbeit präparierten, PCMO nicht beobachtet werden kann. Der LadungsordnungsPhasenübergang lässt sich über den temperaturabhängigen Widerstand mit Hilfe der Methodik, die von Yang et al. etabliert wurde, bestimmen [95]: Für die Auftragung des logarithmischen, spezifischen Widerstandes $\ln (\rho)$ gegen die reziproke Temperatur $1 / T$ existiert eine Änderung des linearen Anstiegs bei der Ladungsordnungs-Temperatur $T_{C O}$ (siehe Abb.16). Dies ist mit einer Änderung der Aktivierungsenergie des Leitungsprozesses gleichzusetzen und wird als Indiz für den Ladungsordnungs-Phasenübergang angesehen [95]. Das in dieser Arbeit präparierte PCMO, hat nach dieser Methode eine Ladungsordnungs-Temperatur von $T_{C O}=220-230 K$, was in guter Übereinstimmung mit dem Bulk-Kristall-Phasendiagramm für eine Dotierung von $x=0.33$ ist [87].

Eine Schichtdickenkontrolle lässt sich durch die Überwachung der deponierten Präkursorlösung-Volumina realisieren. Jedoch ist eine direkte Vorgabe des deponierten Volumen in dem, bis zu dem Zeitpunkt der Präparation, der in diesem Kapitel beschriebenen Proben, einzigen MAD-Setup - MAD 1 (siehe Kap.3.1.2) nicht möglich. Das deponierte Volumen $V_{D e p}$ kann jedoch über die Depositionszeit $t_{D e p}$ (die über die optische Aerosolflussmessung gemessen werden kann) mit $V_{D e p}=\dot{V}_{D e p} \cdot t_{D e p}$ abgeschätzt werden. Allerdings hat dieses Setup mit den peristaltischen Pumpen einen Fehler von bis zu $10 \%$ in der Fließrate $\dot{V}_{D e p}$. Somit ist der Fehler der Schichtdicke auch mit min. 10\% zu veranschlagen. Um diesen Fehler zu minimieren wurde vor jeder Deposition die Flussrate geeicht, indem min. zehn mal die Zeit für den Durchfluss 
4 A-Platz Ordnung in ferromagnetischen halbmetallischen Manganaten

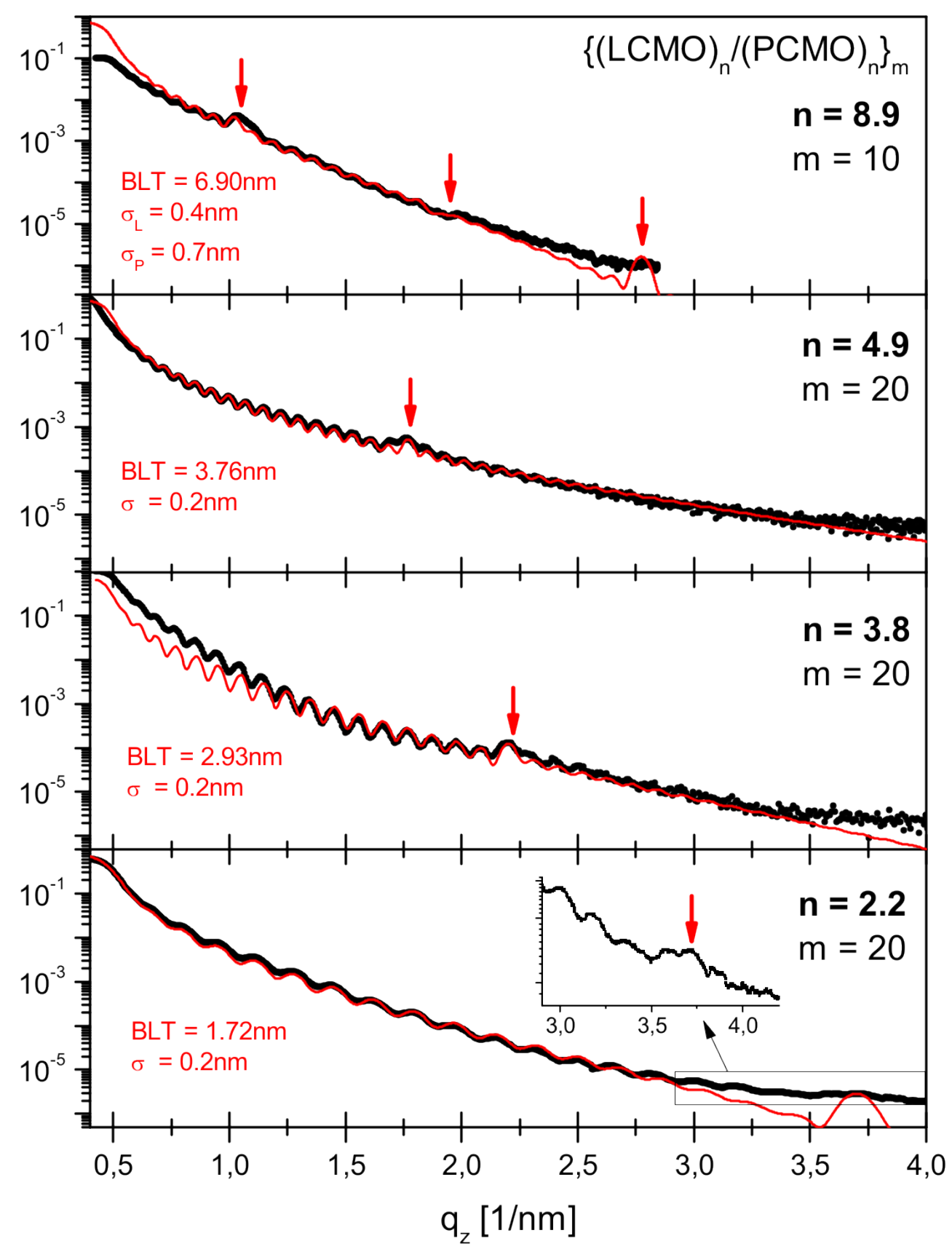

Abbildung 17: Röntgenreflektometriemessungen von $\left(L C M O_{n \text { u.c. }} / P C M O_{n \text { u.c. }}\right)_{m}$ Überstrukturen auf $\mathrm{MgO}(001)$ für verschiedene Bilagen-Dicken $(B L T=2 \cdot n \cdot c)$. Die Überstruktur-Reflexe sind mit roten Pfeilen markiert, und für den $n=2$ Film vergrößert in einem eingebetteten Diagramm dargestellt. Die Messungen wurden mit der Simulationssoftware ReMagX [51] angepasst. Aus den Fit-Parametern erhält man die BLT und die RMS Grenzflächenrauigkeiten $\sigma$. Im oberen Fit für $n=9$ unterscheidet sich die Rauigkeit der Grenzfläche LCMO/PCMO $\sigma_{L}$ von der Grenzfüche PCMO/LCMO $\sigma_{P}$ (mit der Reihenfolge untere/obere Lage). 
von $100 \mu l$ Isopropanol ${ }^{10}$ gemessen und gemittelt wurde. Die Effektivität der Präkursorlösung, d.h. das Verhältnis $d / V$ mit der Schichtdicke des deponierten Filmes $d$ und dem Volumen, der dafür benötigten Präkursorlösung $V$, ist für LCMO und PCMO gleich [77].

Es wurden $\left(\left(\mathrm{La}_{0.67} \mathrm{Ca}_{0.33} \mathrm{MnO}_{3}\right)_{n} /\left(\mathrm{Pr}_{0.67} \mathrm{Ca}_{0.33} \mathrm{MnO}_{3}\right)_{n}\right)_{m}$ (LCMO/PCMO) Übergitter mit nominell $n=2,4,5,9$ und $m=10-20$ Wiederholungen präpariert. Dabei ist die erste Lage aller LCMO/PCMO Übergitter eine 3nm LCMO Pufferschicht um das domainmatching-epitaxy zum Substrat zu gewährleisten. Die Bilagen-Dicke (BLT) konnte mittels XRR-Messungen und -Simulationen mit der Software ReMagX [51] genau auf BLT $/ 2=n=$ 2.2, 3.8, 4.9 bzw. 8.9u.c. bestimmt werden ${ }^{11}$. Die Messungen (schwarz) und Simulationen (rot) sind in Abb.17 aufgetragen. Die Position der Überstrukturreflexe ist mit roten Pfeilen gekennzeichnet. Zusätzlich erhält man aus den Simulationen die Grenzflächenrauigkeiten (als quadratisches Mittel (RMS)) $\sigma$ zwischen den einzelnen Lagen. Diese liegt für die Übergitter mit $n \leq 5$ bei ca. einer halben Monolage mit $\sigma=0.2 n m$. Bemerkenswert ist, dass auch für den $n=2$ Film der Überstrukturreflex klar zu erkennen ist, obwohl der Unterschied der optischen Konstanten zwischen LCMO und PCMO sehr klein ist $(\Delta \delta \sim 0.6 \%$ und $\Delta \beta \sim 4.8 \%$ ). Für den $n=9$ Film sind, aufgrund der großen BLT, drei Überstrukturreflexe in der Messung zu finden und somit mehr Informationen über die Struktur vorhanden. Hier gelingt eine Unterscheidung der Rauigkeit von der Grenzfläche LCMO/PCMO $\sigma_{L}$ und von der Grenzfläche PCMO/LCMO $\sigma_{P}$ (mit der Reihenfolge untere/obere Lage). Es ergibt sich aus der Anpassung der Messkurve eine größere Grenzflächenrauigkeit mit $\sigma<\sigma_{L}=0.4 \mathrm{~nm}<0.7 \mathrm{~nm}=\sigma_{P}$. Um die Anpassung so, wie sie in Abb.17 dargestellt ist, zu bewerkstelligen, muss angenommen werden, dass sich das $\operatorname{Pr} / \mathrm{Ca}$ - leicht (ca. 5\%) von dem $\mathrm{La} / \mathrm{Ca}$-Verhältnis unterscheidet. Hierbei muss jedoch beachtet werden, dass die Größe der Grenzflächenrauigkeit stark von der Wahl der Dotierungsdifferenz abhängt. Da diese nicht genau terminiert werden kann, sind die absoluten Werte von $\sigma$ für alle in Abb.17 gezeigten Messungen nur schwer zu bestimmen. Im Vergleich mit der Oberflächenrauigkeit, ermittelt aus den STM Aufnahmen (weiter unten), stellt die Grenzflächenrauigkeit, die sich für den $n=9$ Film ergibt, ein realistisches Maß für $\sigma$ in den LCMO/PCMO Übergittern auf MgO dar. Aufgrund der geringeren Informationsdichte in den Messungen der Proben mit $n<9$ (nur ein Überstrukturreflex), kann keine Aussage darüber getroffen werden, ob die in der $n=9$ Probe ermittelten strukturellen Eigenschaften (Dotierungsdifferenz, Unterschied in der Grenzflächenrauigkeit), auch auf die Proben mit kleineren $n$ zutreffen. Diese relative scharfen Grenzflächen resultieren auch aus der Tatsache heraus, dass der La-Pr-Diffusionskoeffizient nahe der Depositionstemperatur

\footnotetext{
${ }^{10}$ Das Volumen wurde mit einer Hochpräzisionspipette (rel. Fehler $<1.2 \%$ ) in das System eingegeben; im Fließverhalten gibt keinen Unterschied zwischen DMF und Isopropanol.

${ }^{11}$ In diesen Simulationen kann nur die BLT ermittelt, jedoch nicht die einzelnen Schichtdicken von LCMO und PCMO.
} 
4 A-Platz Ordnung in ferromagnetischen halbmetallischen Manganaten

mit $D\left(945^{\circ} \mathrm{C}\right)<5.3 \cdot 10^{-19} \mathrm{~cm}^{2} / \mathrm{s}$ sehr klein ist. Diese Abschätzung wurde von Felix Massel im Rahmen seiner Bachelorarbeit ermittelt, in der die Änderung der strukturellen und elektrischen Eigenschaften der LCMO/PCMO Übergitter als Funktion der thermischen Auslagerung untersucht wurden [98]. Exemplarisch ist in Abb.14 c) das XRD-Spektrum für den $n=9$ Film dargestellt. Es sind keine Fremdphasenreflexe vorhanden. Die Überstrukturreflexe, die auch nur durch den leichten, bereits angesprochenen, Dotierungsunterschied zwischen LCMO und PCMO erklärt werden können ${ }^{12}$, sind mit -1 und +1 markiert. Die anderen Filme dieser Serie zeigen keine Überstrukturreflexe. Die pseudokubische Gitterkonstante der Überstrukturen liegt nahe der out-of-plane Gitterkonstanten von LCMO, für die verschiedenen Übergitter mit unterschiedlichen $n$, zwischen $0.3866 \mathrm{~nm}$ und $0.3860 \mathrm{~nm}$. Diese Werte zeigen keine systematische Abhängigkeit von $n$. Das STM-Bild des $n=9$ Films (eingebettetes Bild in Abb.14 c) zeigt eine glatte Oberfläche, es sind gitterkonstantengroße Stufen zu erkennen. Die RMS Oberflächenrauigkeit liegt für alle Übergitter zwischen $0.5-0.8 n m$. Zusammenfassend kann festgestellt werden, dass epitaktische LCMO/PCMO Übergitter auf $\mathrm{MgO}(001)$ Substrat präpariert werden konnten. Möglicherweise existiert ein leichter Dotierungsunterschied zwischen LCMO und PCMO. Jedoch wird im Vergleich mit den BulkKristall-Phasendiagrammen [87, 91] keine große Änderung der magnetischen und elektronischen Eigenschaften erwartet. Die RMS Grenzflächenrauigkeit kann auf Werte zwischen 1 - 2u.c. abgeschätzt werden. Daraus folgt, dass insbesondere für Schichten mit kleiner BLT kein reines PCMO und LCMO vorhanden ist. Jedoch ist weiterhin eine chemische Modulation existent, die dieses Material von dem gemischten LPCMO $y=0.5$ Material unterscheidet.

\subsubsection{Elektronische und Magnetische Charakterisierung von $\left(\mathrm{La}_{0.67} \mathrm{Ca}_{0.33} \mathrm{MnO}_{3}\right) /\left(\mathrm{Pr}_{0.67} \mathrm{Ca}_{0.33} \mathrm{MnO}_{3}\right)$ Überstrukturen}

Zunächst ist anzumerken, dass neben den beschriebenen LCMO/PCMO Übergittern auch zwei weitere LPCMO Filme mit $y=0.5$ präpariert wurden, die prinzipiell die ungeordnete Variante der Übergitter mit $n=0$ darstellen. Der Widerstand (bei Nullfeld und bei 20kOe) und die Magnetisierung für einige LCMO/PCMO Übergitter mit verschiedenen $n$ und LPCMO Filme mit verschiedenen $y$, sind als Funktion der Temperatur in Abb.18 bzw. Abb.20 aufgetragen. Die LCMO/PCMO Übergitter mit $n \geq 5$ zeigen einen MIT bei $T_{M I}$ nahe der CurieTemperatur $T_{C} \geq 220 K$, keine thermale Hysterese im Widerstand oder in der Magnetisierung und einen maximalen $\operatorname{CMR}(H)$ in der Größenordnung von $\operatorname{CMR}(20 k O e) \sim 10^{2}-10^{3} \%$. Im Gegensatz dazu weisen die Übergitter mit $n=2$ und $n=4$ einen großen $\operatorname{CMR}(20 k O e) \sim 10^{5}$ - $10^{7 \%}$ und eine thermale Hysterese auf. Darüber hinaus existiert kein MIT bei Nullfeld in

\footnotetext{
${ }^{12}$ Dies kann mit Hilfe der XRD Simulation aus Kap.3.2.2 erschlossen werden.
} 


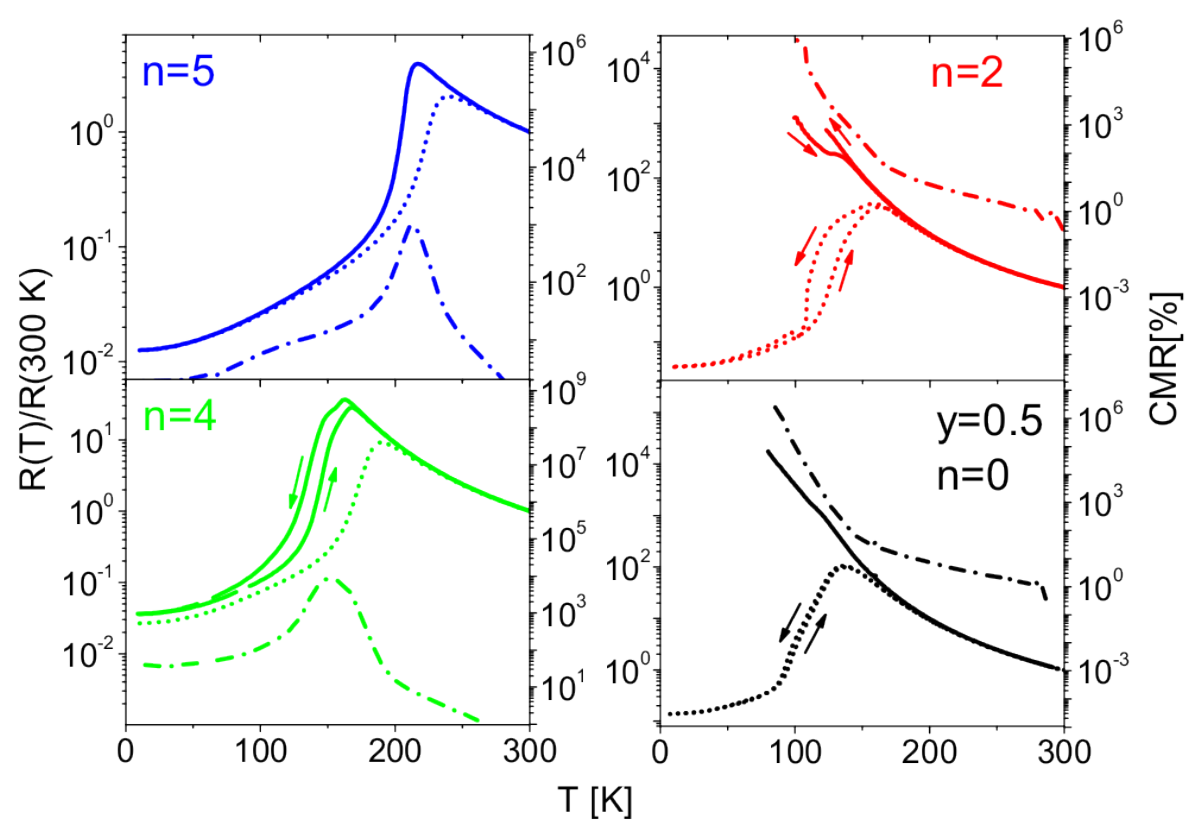

Abbildung 18: Gezeigt sind die CMR(T,20kOe)- (Strichpunktlinie) und die, auf den Widerstand bei $300 K$ normierten, $R(T)$-Kurven bei Nullfeld (durchgezogene Linie) und bei einem externen Feld von 20kOe (gepunktete Linie) für LCMO/PCMO Übergitter mit $n=5$ (blau), 4 (grün), 2 (rot) und $n=0$ bzw. LPCMO mit $y=0.5$ (schwarz). Die Einkühl- und Aufwärmkurven sind mit Pfeilen markiert.

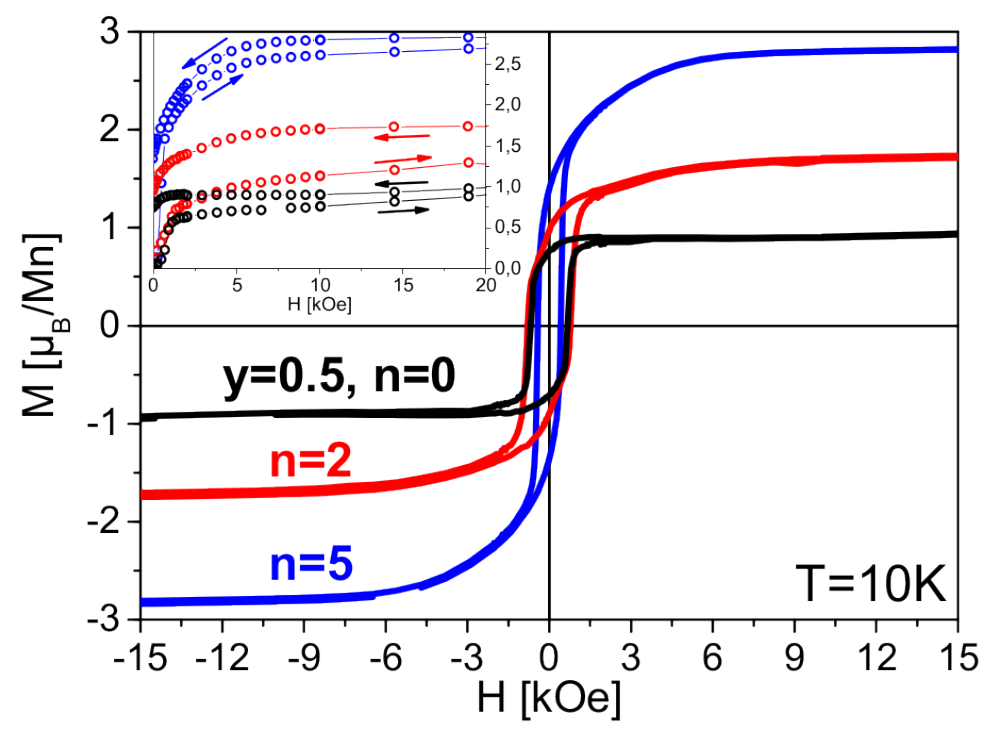

Abbildung 19: $M(H)$-Kurven nach der Einkühlung bei Nullfeld auf $10 K$ von LCMO/PCMO $\ddot{U}$ bergittern mit $n=5$ (blau), $n=2$ (rot) und $n=0$ bzw. LPCMO mit $y=0.5$. Das eingebettete Diagramm zeigt die Neukurven zwischen 0 und 20kOe. Für diese Messungen wurde ein maximales Feld von 50kOe angelegt. 
4 A-Platz Ordnung in ferromagnetischen halbmetallischen Manganaten

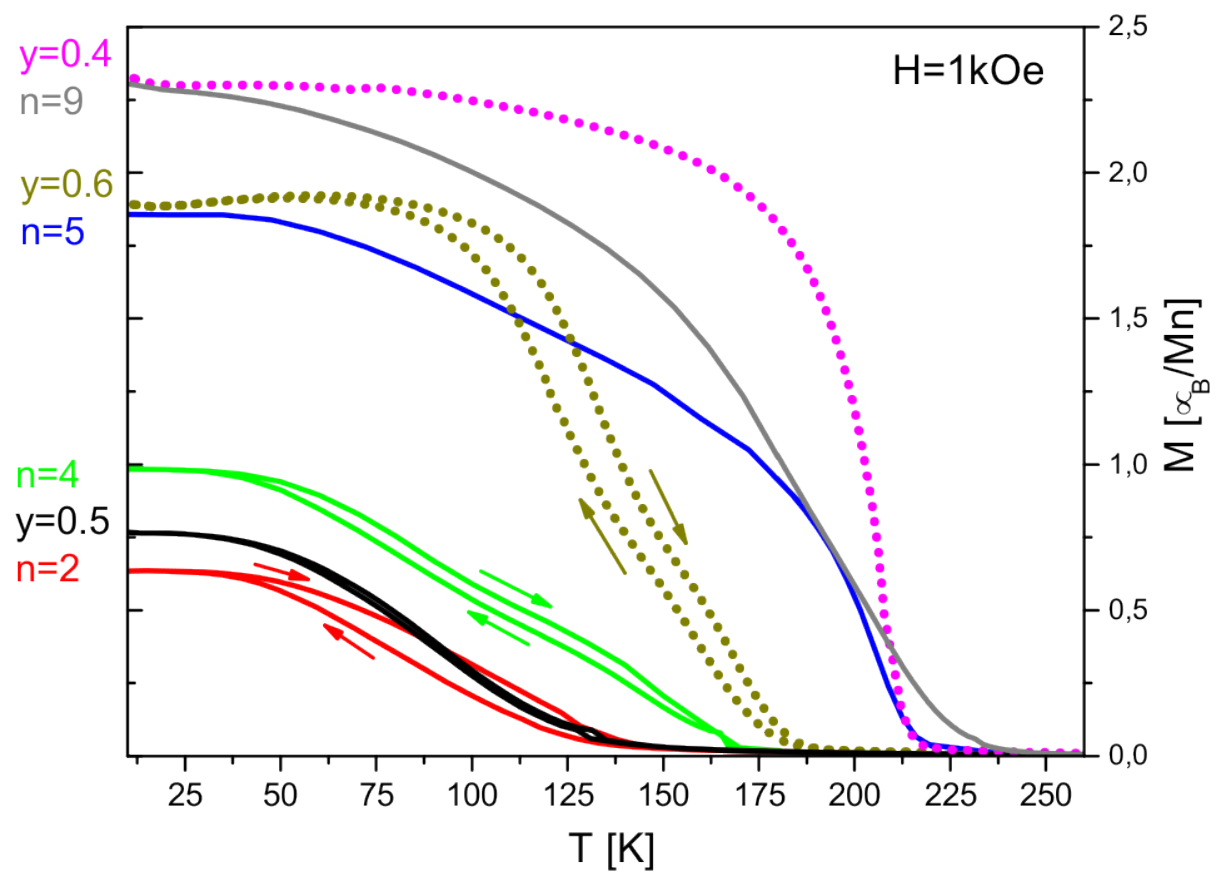

Abbildung 20: $M(T)$-Kurven bei einem externen Feld von $1 k O e$ von LCMO/PCMO Übergitter (durchgezogene Linie) mit $n=9$ (grau), 5 (blau), 4 (grün), 2 (rot) und LPCMO-Filme (gepunktete Linie) mit $y=0.4$ (magenta) und 0.6 (ocker). Einkühl- und Aufwärmkurven sind mit Pfeilen markiert.

dem $n=2$ Übergitter. Ähnlich dazu sind die elektronischen Eigenschaften der LPCMO Filme mit $y=0.5$. Sie zeigen keinen $T_{M I}$ bei Nullfeld, einen hohen CMR, jedoch, im Unterschied zu dem Übergitter mit $n=2$, nur ein sehr schwaches hysteretisches Verhalten. Als Funktion der BLT fällt das $T_{M I}$ und damit auch $T_{C}$ der Übergitter mit steigendem $n$ ab, die Tendenz ist vergleichbar mit dem LPCMO System mit steigender Pr-Dotierung y. In Abb.19 ist die Magnetisierung $M$ als Funktion des externen Feldes $H$ mit einem maximalen angelegten Feld von $50 k O e$, für drei LCMO/PCMO Übergitter nach dem Einkühlen bei Nullfeld auf $10 \mathrm{~K}$ dargestellt. Mit kleiner werdenden BLT sinkt die Sättigungsmagnetisierung von $2.8 \mu_{B} / M n$ bei $n=5$ auf eine Wert von $\sim 0.9 \mu_{B} / M n$ bei $n=0$, bzw. im LPCMO Sys-tem $y=0.5$, stetig ab. Gleichzeitig steigt das Koerzitivfeld mit $n$ weiter, bis zu einem Wert von $8000 e$ an, wobei das $H_{c}$ von dem $n=2$ Übergitter leicht größer ist als das von dem $y=0.5$ LPCMO Film. Zusätzlich sind die Neukurven dieser Messungen in dem eingebetteten Diagramm in Abb.19 dargestellt. Die Differenz zwischen der Feldauf- und Feldab-Kurve ist für das $n=2$ Übergitter signifikant größer im Vergleich zu dem $n=5$ Übergitter und dem $y=0.5$ LPCMO Film.

Um Aussagen über den PS Zustand treffen zu können, wurde die Relaxation des elektrischen Widerstandes nach dem Anlegen eines externen Feldes gemessen. Diese Messungen wurden in einem optischen Kryostat (Displex 202E von Advanced Research Systems) mit 
4.1 Künstliche elektronische Phasenseparation in teilgeordneten LPCMO

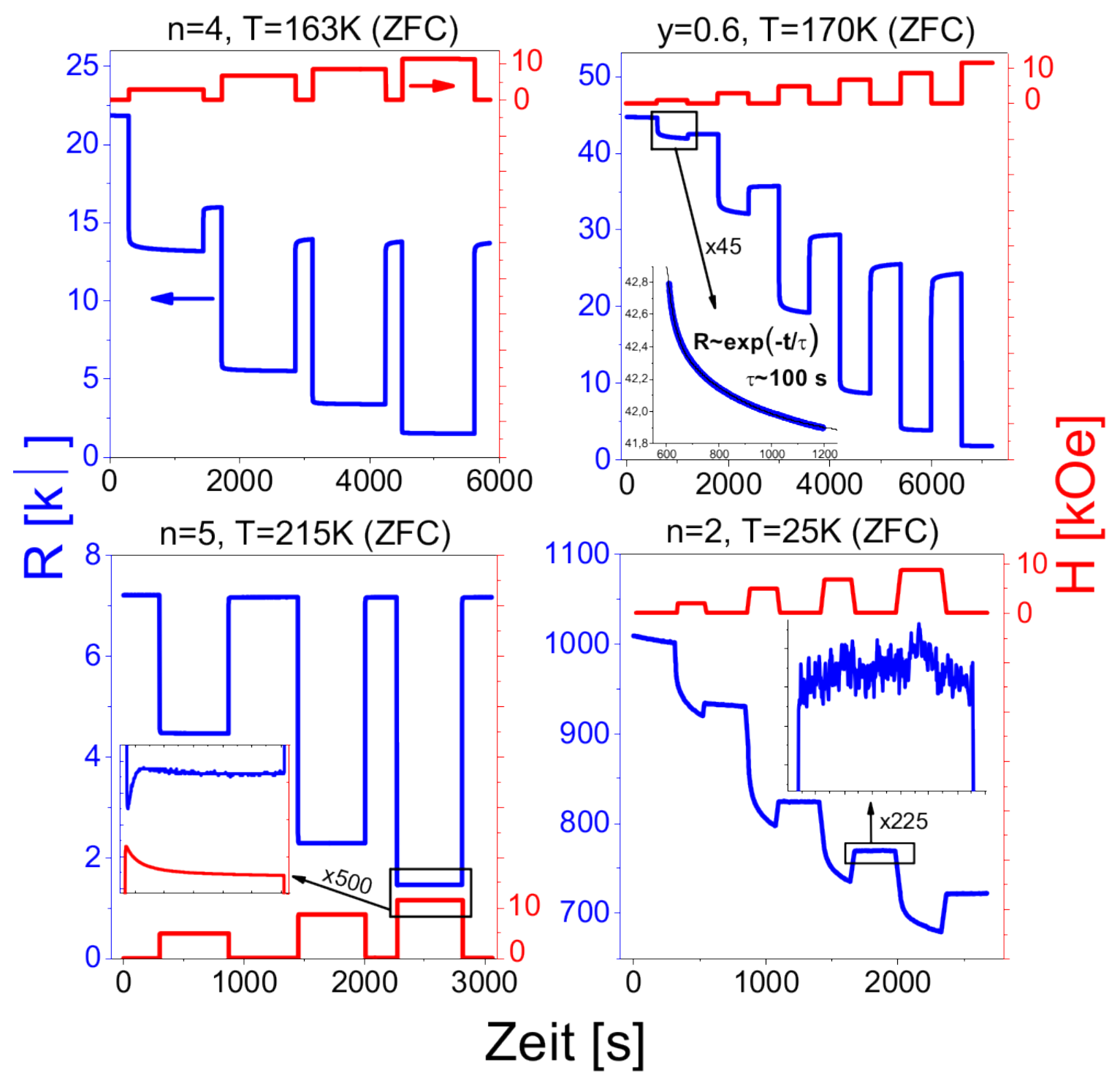

Abbildung 21: Relaxation des elektrischen Widerstandes $R$ durch das Schalten eines externen Feldes $H$ für die LCMO/PCMO Übergitter mit $n=5$, 4, 2 sowie einem LPCMO-Film mit $y=0.6$, nach einer Abkühlung zu der, über dem entsprechenden Diagramm angegebenen, Temperatur bei Nullfeld (ZFC). Die eingebetteten Diagramme zeigen die Vergrößerung des entsprechend markierten Bereiches. Die Daten sind aus [99] entnommen. 
4 A-Platz Ordnung in ferromagnetischen halbmetallischen Manganaten

einem geschlossenen He-Kühlkreislauf, der sich in einem konventionellen Elektromagneten (mit einem maximalen magnetischen Fluss $B_{\max } \sim 1.5 T$ ) befindet, durchgeführt. Über eine Vierpunktmessung auf der Probenoberfläche wurde ein Strom von $100 \mu A$ angelegt und die Spannung mit einem Multimeter des Typs Keithley 197 gemessen. Diese Messungen wurden von Felix Koeth im Rahmen seiner Bachelorarbeit [99] durchgeführt. Aufgrund der langreichweitigen PS im LPCMO System gibt es metastabile Zustände. Diese sind durch eine Hierarchie von Energiebarrieren, die durch elastische Deformationen im Kristall induziert werden, von dem Gleichgewichtszustand getrennt. Legt man nun ein externes Magnetfeld an diesen metastabilen Zustand an, relaxiert das System langsam in den Gleichgewichtszustand [100, 101]. An diesem Punkt muss noch angemerkt werden, dass das Schalten des Magneten in einem wesentlich kleineren Zeitraum abläuft, als die Widerstands Relaxation mit typischen Werten im Bereich von mehreren Sekunden [99, 101]. Die wichtigsten Ergebnisse dieser Messungen an LCMO/PCMO Übergittern und an LPCMO Filmen sind in Abb.21 dargestellt. Um den metastabilen Zustand zu erreichen wurden die Proben zunächst in Nullfeld auf Temperaturen nahe $T_{M I}$ abgekühlt. Für LPCMO mit $y=0.6$ wird eine Relaxation mit einer typischen Relaxationszeit von $\tau \sim 100 s$ und eine Rückrelaxation, nach dem Abschalten des Feldes, beobachtet. Diese Beobachtung ist übereinstimmend mit den Resultaten in Bulk-LPCMO Kristallen [101]. Genau das gleiche Verhalten beobachtet man in den LCMO/PCMO Übergittern mit $y=4$. In dem $n=2$ Übergitter ist das Relaxationsverhalten, wie in den bisher beschriebenen Proben nicht beschränkt auf einen kleinen Temperaturbereich um $T_{M I}$, stattdessen ist es für $T<90 \mathrm{~K}$ immer vorhanden (in Abb.21 exemplarisch für $T=25 \mathrm{~K}$ gezeigt). Zusätzlich existiert keine Rückrelaxation in dieser Probe. LCMO/PCMO Übergitter mit $n \geq 5$ zeigen keine langsame Relaxation. Die hier beobachtete schnelle Relaxation kann dem Abklingen des externen Feldes zugeschrieben werden.

\subsubsection{Diskussion und Vergleich der künstlichen und intrinsischen elektronischer Phasenseparation in LCMO/PCMO und LPCMO}

Die Daten aus den Relaxationsmessungen der Übergitter mit $n=2$ als auch $n=4$ zeigen, dass ein PS-Zustand vorhanden ist, der, aufgrund der Messgeometrie, auch senkrecht zu den einzelnen Lagen der Übergitterstruktur existiert. Dabei verbleibt die $n=2$ Probe nach dem Entfernen des externen Feldes in einem großskaligen PS-Zustand (keine thermale Rückrelaxation), der über die Geometrie der künstlichen PS durch die Übergitterstruktur hinaus geht. Das bedeutet, es existiert eine elektrische PS, die nicht aus der chemischen PS durch die Aufteilung in LCMO- und PCMO-Lagen entsteht. In den $n \geq 5$ Übergittern hingegen existiert kein metastabiler- und damit auch kein PS-Zustand parallel zur Messrichtung 
des elektrischen Widerstandes. Zusammenfassend können die LCMO/PCMO Übergitter damit in zwei Gruppen aufgeteilt werden, deren Grenze zwischen $n=5$ und $n=4$ liegt mit $n_{\text {krit }} \sim 4.5$.

Oberhalb dieser Grenze erkennt man in den Widerstandsmessungen, dass der Phasenübergang vom paramagnetischen Isolator (PMI) zum FMM nahe der 2.Ordnung ist (es ist keine thermische Hysterese zu erkennen, moderater CMR, keine langsame Relaxation). Demzufolge entspricht dieser Phasenübergang, einem PMI/FMM Übergang, wie man ihn in LCMO erwarten würde. Auch das $T_{C} \sim T_{M I} \sim 220$ - 230K liegt nahe (etwas unterhalb) der LCMO Übergangstemperatur von $T_{C} \sim 270 K$. In Anbetracht der Richtung der Messung des elektrischen Widerstandes (parallel zur Filmoberfläche), kann aus dieser Beobachtung geschlossen werden, dass die elektrische Leitung bei tiefen Temperaturen vornehmlich von den LCMO Lagen getragen wird. Jedoch kann nicht ermittelt werden, ob das PCMO in diesen Übergittern isolierend ist, da in den $R(T)$ Kurven kein isolierendes Verhalten bei tiefen Temperaturen erkennbar ist. Es muss an dieser Stelle jedoch erwähnt werden, dass leitfähige Kurzschlüsse durch das mit La andotierte PCMO Material (aufgrund der Grenzflächenrauigkeit) oder FMM-Domänen durch eine elektrische PS in den PCMO Lagen vorhanden sind, die verhindern würden, dass ein isolierendes Verhalten von PCMO beobachtet werden kann. Die Sättigungsmagnetisierung ist für das gesamte Übergitter im Vergleich zu den LCMO Filmen reduziert, allerdings nicht so stark, wie man es von zwei nicht gegenseitig beeinflussten LCMO und PCMO Bestandteilen erwarten würde: Nach Abb.15 ist die Sättigungsmagnetisierung von LCMO $M_{s, L C M O} \sim 3.6 \mu_{B} / M n$ und von PCMO (von dem FM-Anteil) $M_{s, P C M O} \sim 0.5 \mu_{B} / M n$. Somit ist die Sättigungsmagnetisierung für ein Übergitter, dass aus gleichen, nicht wechselwirkenden Bestandteilen LCMO und PCMO besteht $M_{s, L P-S L} \sim 0.5 \cdot 3.6 \mu_{B} / M n+0.5 \cdot 0.5 \mu_{B} / M n \sim 2 \mu_{B} / M n$. Aus den experimentellen Daten lässt sich schließen, dass die Magnetisierung des PCMO im Vergleich zum einphasigen Film stark erhöht ist ${ }^{13}$. Eine Vergrößerung der Bandbreite von PCMO an der Grenzfläche zum LCMO durch die Eindotierung von La und/oder Verspannung durch das LCMO verursacht eine Erhöhung des FMM-Anteils in PCMO [74, 75] und damit auch der Sättigungsmagnetisie-rung. Dies hat zur Folge, dass gleichzeitig die Bandbreite von LCMO gesenkt wird, was die verringerte Übergangstemperatur erklären kann. In LCMO/PCMO Übergittern mit $n \leq 5$ zeigen die einzelnen Manganate (LCMO und PCMO) eine geringe gegenseitige Wechselwirkung. Explizit liegt in diesen Übergittern keine von der chemischen PS losgelöste elektrische PS vor, was durch die Relaxationsmessungen gezeigt wird. Während die Bandbreite des PCMOs gegenüber den Bulk-Eigenschaften scheinbar erhöht ist, ist die

\footnotetext{
${ }^{13}$ Die Annahme, dass LCMO eine Sättigungsmagnetisierung $M_{s}>3.6 \mu_{B} / M n$ besitzt, ist ohne experimentelle Bestätigung durch eine ortsaufgelöste Messung der Magnetisierung aus Neutronen- oder Röntgenstreuung, nicht haltbar.
} 
4 A-Platz Ordnung in ferromagnetischen halbmetallischen Manganaten

Bandbreite von LCMO verringert. Diese Wechselwirkung wird dabei durch die gegenseitige Verspannung und/oder Mischdotierungen an der Grenzfläche vermittelt.

Unterhalb der Grenze von $n_{k r i t} \sim 4.5$ u.c. existiert eine starke gegenseitige Wechselwirkung der Manganate. Wie sich aus den Relaxationsmessungen erschließen lässt, sind speziell diese Proben geprägt von einer elektronischen PS, die geometrisch von der chemische PS unabhängig ist und über diese hinausgeht. Zusätzlich zeigt der PMI/FMM Übergang mit der Existenz einer großen thermalen Hysterese die Eigenschaften eines Phasenübergangs erster Ordnung, der eine Voraussetzung für die elektronische PS ist. Mit sinkender BLT verringert sich die Gesamtmagnetisierung und damit der Anteil der FMM-Phase in diesen Übergittern. Für $n=2$ ist der Anteil der FMM-Phase so klein, dass kein MIT bei Nullfeld vorhanden ist. Damit liegt die Perkolationsgrenze in den Übergittern offensichtlich zwischen $n=2$ und 4. Wie auch LPCMO nahe der Perkolationsgrenze [77] zeigen die Übergitter im PS-Zustand einen großen CMR: Wird ein externes Feld an diesen FMM/COO-PS-Zustand angelegt, sinkt der Widerstand drastisch durch das teilweise Aufschmelzen der COO-Phase und die parallele Ausrichtung der FMM-Domänen ab [78, 90]. Dabei ist der CMR-Effekt für Proben, die unterhalb der Perkolationsgrenze mit $y<y_{c}$ (Proben mit Nullfeld-MIT) anzusiedeln sind tendenziell reversibel und beschränkt auf einen Temperaturbereich nahe $T_{C}$. Für Proben oberhalb dieser Grenze ist der CMR irreversibel über einen großen Temperaturbereich $T<T_{C}$ vorhanden [77]. Diese Charakteristika sind in den Übergittern auch klar existent und deutlich in den Widerstandskurven zu erkennen (siehe Abb.18). Auch das irreversible Aufschmelzen der COO-Phase kann in dem $n=2$ Übergitter anhand der Neukurve der Magnetisierung beobachtet werden. Die große Differenz zwischen der Feldauf-FeldabKurve für das $n=2$ Übergitter deutet darauf hin, dass das maximale Feld von $50 k O e$ in dieser Messung (siehe Abb.19) ausreicht, um einen signifikanten Anteil der COO-Phase in eine FMM-Phase umzuwandeln. Bereits ein Feld von 20kOe erzeugt einen genügend großen Anteil von FMM-Domänen um einen MIT in dieser Probe zu induzieren. Wie in den $M(H)$ Kurven zu erkennen ist, ist diese COO/FMM Phasenumwandlung irreversibel und führt zu dem ebenso irreversiblen CMR. Generell entsprechen die Übergitter mit $n<n_{\text {krit }}$ in ihren elektrischen und magnetischen Eigenschaften LPCMO Filmen mit $y \sim 0.6-0.8$ nahe der Perkolationsgrenze $y \sim 0.75$. Insbesondere können die physikalischen Eigenschaften in diesen Übergittern nicht mehr durch die Bulk-Eigenschaften von schwach wechselwirkenden LCMO/PCMO Lagen erklärt werden.

Eine derartige Grenze $n_{k r i t}$ wurde auch in künstlich phasenseparierten LSMO/PCMO Übergittern beobachtet, die verspannt auf $\mathrm{SrTiO}_{3}$-Substraten gewachsen wurden [12]. Erstaunlicherweise entspricht diese Längenskala mit $n_{k r i t} \cdot 0.386 \mathrm{~nm} \sim 1.7 \mathrm{~nm}=r_{C P}$ der Größen- 
4.1 Künstliche elektronische Phasenseparation in teilgeordneten LPCMO

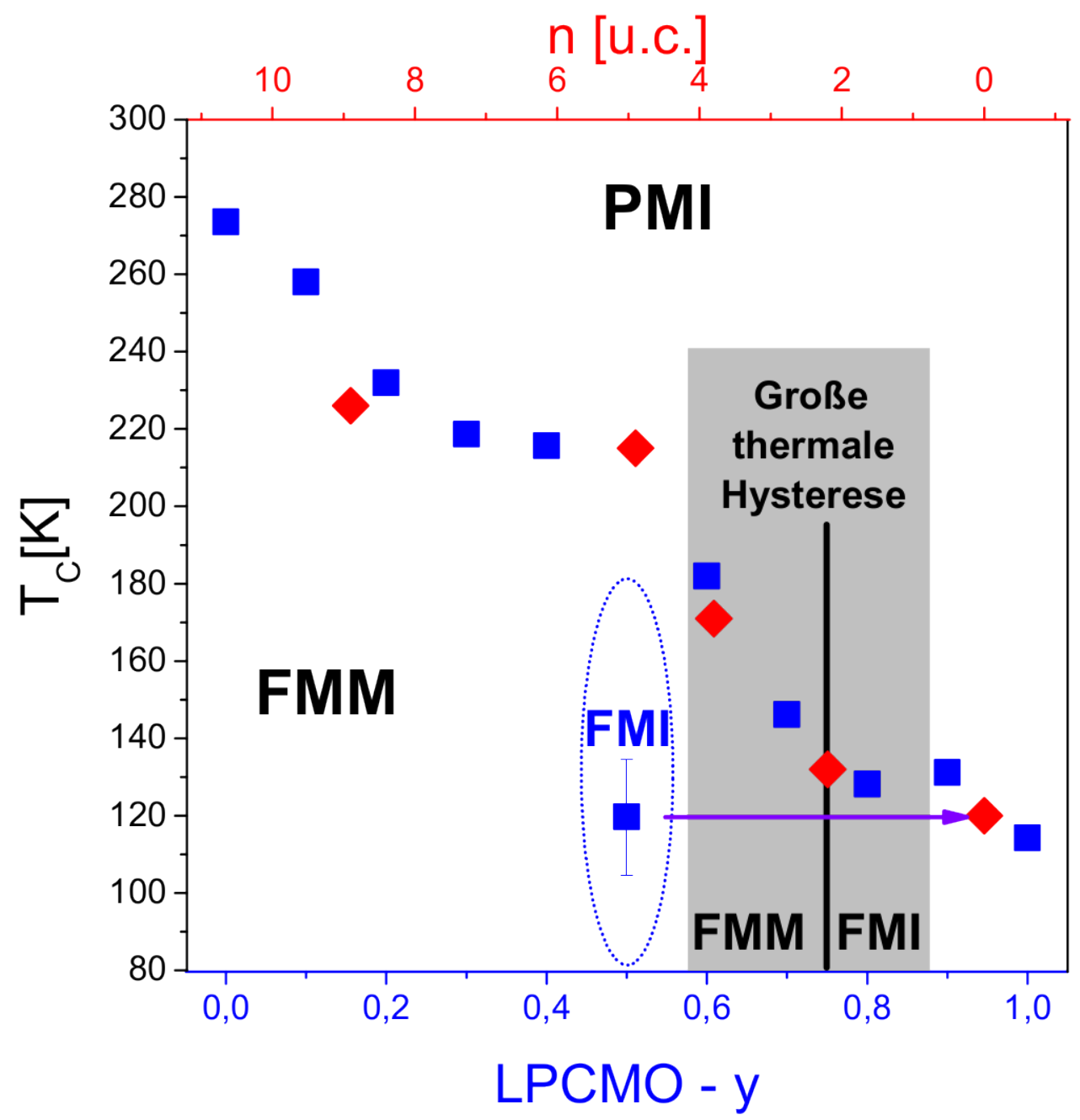

Abbildung 22: Phasendiagramm der LPCMO Filme in Abhängigkeit von dem Pr-Gehalt y (blaue Quadrate) aus [77] (die Daten aller in dieser und in [77] erstellten $y=0.5$ LPCMO Filme wurden berücksichtigt und zusammengefasst). Dazu ist das Phasendiagramm der LCMO/PCMO Übergitter superpositioniert mit $n(y)=10.6-11.3 \cdot y$ u.c. (rote Rauten). Bereiche mit ferromagnetisch metallischen (FMM), paramagnetisch isolierendem (PMI) als auch ferromagnetisch isolierendem (FMI) Verhalten bei Nullfeld sind markiert. Das graue Rechteck stellt das Intervall in den Phasendiagrammen dar, indem die Proben eine ausgeprägte, thermale Hysterese zeigen. Die Perkolationsgrenze ist als schwarze Gerade bei $y \sim 0.75$ dargestellt. Die Konvergenz von LPCMO y $=0.5$ und LC$M O / P C M O n=0$ ist mit dem violetten Pfeil gekennzeichnet. 
4 A-Platz Ordnung in ferromagnetischen halbmetallischen Manganaten

ordnung der korrelierten Polaronen [84, 85]. Diese Feststellung liefert einen Erklärungsansatz für die eben erwähnte Grenze: Die maximale relative Anzahl der korrelierten Polaronen $n_{C P, \max }$ ist in dem LPCMO System direkt abhängig von dem Pr-Gehalt $y$ [78]. Ein größerer $P r$-Gehalt führt zu einen größeren $n_{C P, \max }$. Ist die Schichtdicke von PCMO $d$ in den Übergittern kleiner als die Größenordnung der korrelierten Polaronen $d>r_{C P}$, so gibt es zwei Szenarien, die sich ergeben können: Entweder breiten sich die korrelierten Polaronen in das polaronen-ärmere LCMO aus oder es existieren durch die geometrische Beschränkung wesentlich weniger Polaronen im Material. Anhand der beobachteten physikalischen Eigenschaften ist jedoch ein Anstieg des AFM-COO Teilvolumens zu vermuten, wodurch der erste vorgeschlagene Fall realistisch erscheint, in dem sich die Polaronen in das LCMO ausbreiten.

In dieser Arbeit können darüber hinaus die physikalischen Eigenschaften der Übergitter mit denen der LPCMO-Bulk Filme, die mit der gleichen Depositionsmethode auf dem gleichen Substratmaterial gewachsen wurden, verglichen werden. In Abb.22 ist die Übergangstemperatur der LCMO/PCMO Übergitter als Funktion von $n$ auf das LPCMO-Phasendiagramm aus [77] mit der empirischen Abhängigkeit $n(y)=10.6$ - 11.3 •y u.c. gelegt (in diesem Phasendiagramm sind ergänzend die Daten, der in dieser Arbeit erstellten LPCMO $y=0.5$ Filme eingegangen). Bemerkenswerterweise stimmen nicht nur die Übergangstemperaturen für diese $n(y)$-Abhängigkeit überein, sondern auch die restlichen magnetischen und elektrischen Eigenschaften: die Größe der thermalen Hysterese (aufgrund des Phasenüberganges erster Ordnung), das Relaxationsverhalten, die Lage der Perkolationsgrenze, die Größenordnung und der Temperaturbereich des CMR-Effektes (Vergleiche mit Abb.18, 19, 20, 21 und Literatur [77]). Speziell die große Ähnlichkeit der physikalischen Eigenschaften der LPCMO y $=0.6$ und LCMO/PCMO $n=4$ Proben ist dabei hervorzuheben. Es existieren auch wahrnehmbare Unterschiede zwischen diesen beiden Systemen, z.B. ist der PM/FM Phasenübergang der Übergitter im Vergleich zu den einphasigen Filmen wesentlich breiter (siehe Abb.20). Die wesentliche Ursache dafür lässt sich möglicherweise in der größeren Varianz der Bandbreite $\sigma(t)$, verursacht durch die Übergittermodulation, finden. Unter der vorgeschlagenen $n(y)$-Abhängigkeit, verschiebt sich das $y=0.5$ LPCMO, unter der Berücksichtigung, dass dieser auch das ungeordnete $n=0$ Übergitter darstellt, zu einem Punkt nahe $y \sim 0.95$ (in Abb.22 angedeutet durch den violetten Pfeil). Dieser Punkt im LPCMO Phasendiagramm repräsentiert die physikalischen Eigenschaften der $y=0.5$ bzw. $n=0$ Filme sehr gut: LPCMO Filme in diesem Bereich des Phasendiagrammes zeigen eine kleine thermale Hysterese, einen großen irreversiblen CMR und eine verringerte Sättigungsmagnetisierung $M_{s} \sim 0.5 \mu_{B} / M n$ [77]. Es ist folglich möglich die Phasendiagramme von dünnen LPCMO Filmen und LCMO/PCMO Übergittern mit der beschriebenen $n(y)$-Abhängigkeit kongruent übereinanderzulegen. Dabei ist der Konvergenzpunkt $y=0.5$ bzw. $n=0$ an zwei verschiede- 
nen Stellen in diesem kombinierten Phasendiagramm vorhanden. Diese Separation kann nur kontrovers gedeutet werden. Ungeachtet davon bewegt sich das teilgeordnete LCMO/PCMO Übergittersystem mit kleiner werdenden $n$ stetig auf die physikalischen Eigenschaften der $n=0$ bzw. $y=0.5$ Proben zu. Die erstaunliche Folgerung ist, dass es ohne Änderung der chemischen Gesamtkomposition möglich ist, LPCMO Filme zu synthetisieren, welche die physikalischen Eigenschaften von nahezu dem gesamten LPCMO Phasendiagramm, unter der Variation des Pr-Gehaltes $y$, abdecken. In diesem Zusammenhang ist die Teilordnung der La bzw. Pr-Ionen durch die Präparation von Übergittern, der Mechanismus zur Kontrolle der magnetischen und elektrischen Eigenschaften. Mit der Hilfe dieser Beobachtungen kann auch das LPCMO mit $y=0.5$ weiter diskutiert werden. Im Vergleich mit seinen Nachbardotierungen scheint das LPCMO $y=0.5$ bei tiefen Temperaturen einen viel größeren relativen Volumenanteil der AFM-COO-Phase zu besitzen, sodass es vergleichbare Eigenschaften, wie hochdotiertes LPCMO besitzt. Es stellt sich automatisch die Frage, warum die AFM-COOPhase in diesem Material bevorzugt ist: Es wurde gezeigt, dass die lagenweise Ordnung von La und $\operatorname{Pr}$ in den LCMO/PCMO Übergittern zu einer Reduktion des Volumenanteils der AFM-COO-Phase führt. In der Literatur wurde bereits gezeigt, dass A-Platz Ordnung einen großen Einfluss auf die elektronische Phasenseparation haben kann [4]. Es ist somit vorstellbar, dass sich in dem LPCMO $y=0.5$ System eine A-Platz Ordnung einstellt, die sich von der lagenweisen Ordnung unterscheidet und dazu führt, dass sich die physikalischen Eigenschaften von dem ungeordneten Fall [74, 76] unterscheidet und eine COO-Phase präferiert. Eine Verstärkung der COO-Phase durch die A-Platz Ionen Ordnung in stark Sr-dotierten LSMO [9] und eine selbstorganisierte Ordnung von La und $C a$ in LCMO [8] konnte bereits beobachtet werden. Diese Beobachtungen unterstützen die hier aufgestellte These, die mit der Hilfe einer sehr aufwendigen TEM Investigation (da die genaue Struktur dieser Ordnung nicht bekannt ist) experimentell untermauert werden könnte. Zusätzlich würde diese Untersuchung Aufschluss über den Typ und die Ausprägung der COO-Phase in diesem Material geben [102]. 



\subsection{Künstliche A-Platz Ordnung in $\mathrm{La}_{0.67} \mathrm{Sr}_{0.33} \mathrm{MnO}_{3}$}

Bereits in Kap. 2.3 wurde herausgestellt, dass eine große Differenz zwischen der maximalen theoretisch möglichen Curie-Temperatur von 530K [7] und der bisher höchsten experimentell erreichten Curie-Temperatur von $\sim 370 \mathrm{~K}$ [31] in $\mathrm{La}_{0.67} \mathrm{Sr}_{0.33} \mathrm{MnO}_{3}$ (LSMO) existiert. Diese Differenz kann auf Verspannung zurück geführt werden, die aus der geometrischen Fehlanpassung der A- und B-Platz Ionen resultiert. Die Verspannung kann phänomenologisch in zwei Beiträge aufgeteilt werden [7]: Es existiert ein Beitrag, der quadratisch von dem Mittelwert der Fehlanpassung abhängt. Er beschreibt die mittlere Verspannung durch die Fehlanpassung im gesamten Gitter und wird durch den TF ausgedrückt. Des Weiteren existiert ein Beitrag, der linear von der Varianz der Fehlanpassung bzw. dem Größenunterschied der A-Platz Ionen abhängt. Dieser Beitrag beschreibt die Fluktuation der Verspannungen an verschiedenen Punkten im Gitter durch die Unordnung der unterschiedlich großen A-Platz Ionen. Beide Beiträge werden durch Gleichung (4) in Kap. 2.3 erfasst. Wendet man diese Gleichung mit den empirischen Faktoren $p_{1}$ und $p_{2}$ aus [7] auf LSMO mit den Ionenradien aus [103] an, reduziert sich unter Berücksichtigung des TFs das maximale, theoretische $T_{C}$ auf $435 K$ für eine Varianz von $\sigma^{2}=0$. Folglich führt die Unordnung der Verspannung bzw. der A-Platz Ionen $L a$ und $S r$ zu einer Reduktion der Übergangstemperatur von $\sim 65 K$. Im Umkehrschluss kann gefolgert werden, dass eine Kationen-Ordnung zu einer Erhöhung der Übergangstemperatur führen kann.

Experimentell konnte diese These von Moshnyaga et al. bestätigt werden [8]: Mittels MAD wurden diverse $\mathrm{La}_{0.75} \mathrm{Ca}_{0.25} \mathrm{MnO}_{3}$ Filme auf $\mathrm{MgO}$ (100) gewachsen. Innerhalb dieser Serie unterscheidet sich der PMI/FMM Phasenübergang qualitativ und kann quantitativ durch die Übergangstemperatur $T_{M I} \sim T_{C}$ (Position des Phasenüberganges) und dem $\alpha_{R}$ Wert (Schärfe des Phasenüberganges) beschrieben werden. Dabei ist die Übergangstemperatur korreliert mit der Schärfe des Phasenübergangs. Der spezifische Widerstand und die Magnetisierung als Funktion der Temperatur ist in Abb.23 a) bzw. b) für zwei Proben dieser Serie dargestellt. Wie weiter unten im Text genauer ausgeführt, unterscheiden sich die Proben dahingehend, dass ein Film eine Kationen-Ordnung zeigt (O-Film, in blau in Abb.23 a und b), während der andere ungeordnet ist (D-Film, in rot in Abb.23 a und b). Der O-Film zeigt gegenüber dem D-Film eine um $8 K$ höhere Übergangstemperatur und einen schärferen Phasenübergang, zu sehen anhand der eingebetteten Diagramme in Abb.23 a) und b) für den $\alpha_{R^{-}}$und $\alpha_{M^{-}}$Wert $^{14}$. Überdies ist der CMR des O-Films leicht erhöht und der spezifische Widerstand des O-Films, sowohl bei hohen als auch bei niedrigeren Temperaturen, niedriger im Vergleich zum D-Film. TEM Messungen des O-Films zeigen eine A-Platz Kationen-Ordnung,

\footnotetext{
${ }^{14}$ Der $\alpha_{M}$-Wert ist analog zum $\alpha_{R}$-Wert definiert.
} 
4 A-Platz Ordnung in ferromagnetischen halbmetallischen Manganaten
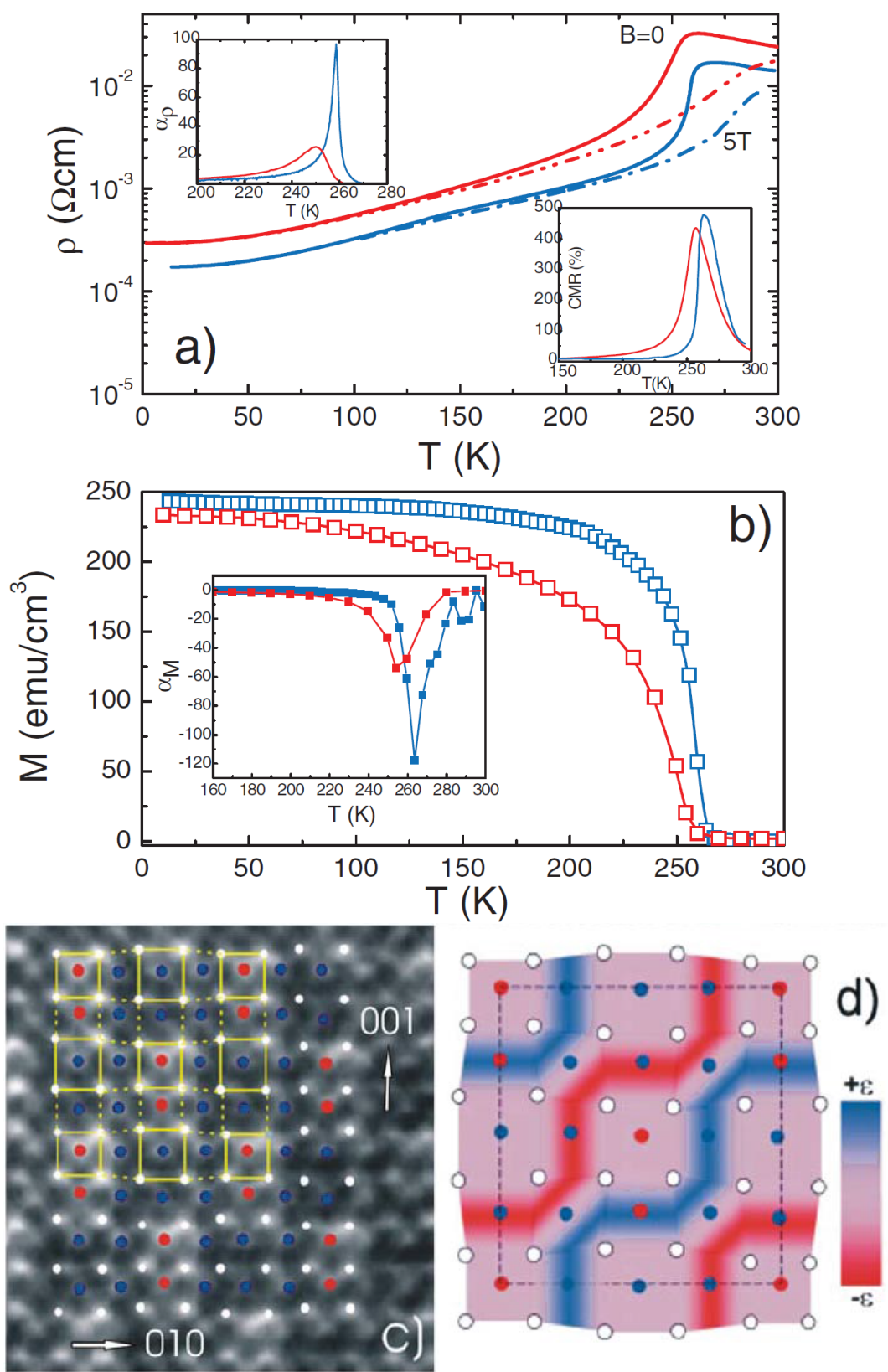

Abbildung 23: a) Temperaturabhängigkeit des spez. Widerstandes $\rho(T)$ für einen A-Platz geordneten $\mathrm{La}_{0.75} \mathrm{Ca}_{0.25} \mathrm{MnO}_{3}$ (blau) und einen ungeordneten $\mathrm{La}_{0.75} \mathrm{Ca}_{0.25} \mathrm{MnO}_{3}$ (rot) Film für externe Felder von $H=0 O e$ (geschlossene Linie) und $H=50 k O e$ (Punkt-Strich-Linie). Die eingebetteten Diagramme zeigen den CMR- und den $\alpha_{\rho}$-Wert. b) Abkühlkurven der Magnetisierung für die Proben wie in a) bei einen externen Feld von $100 O e$. Das eingebetteten Diagramm zeigt den $\alpha_{M}$-Wert. c) Hoch aufgelöste TEM Aufnahme der Überstruktur in dem geordneten $\mathrm{La}_{0.75} \mathrm{Ca}_{0.25} \mathrm{MnO}_{3}$ Film. Die Arten der Ionen sind zur besseren Kenntlichkeit farbig markiert: Mn-weiß, La-blau, Ca-rot. d) Die lokale Verteilung der strukturellen Verspannung $\epsilon$ in der Überstruktur: Druckspannung-rot, Zugspannung-blau. Die Diagramme sind aus [8] entnommen. 
die in eine kubische Überstruktur von $4 \times 4$ pseudokubischen Perowskit-Einheitszellen resultiert. Wie in Abb.23 c) dargestellt, sind dabei die La (blau) bzw. Ca (rot) Ionen entlang der [001] Richtung eindimensional aufgereiht. In der [010] Richtung wechseln sich $\mathrm{LaMnO}_{3}$ mit $\mathrm{La}_{0.5} \mathrm{Ca}_{0.5} \mathrm{MnO}_{3}$ Lagen ab, wobei in den zuletzt genannten Lagen jedes A-Platz Ion in der [001] Richtung sowohl ein $C a$ als auch ein $L a$ Ion als Nachbarn besitzt. Die aus der Kation-Ordnung resultierenden Struktur der Verspannung des Kristallgitters ist in Abb.23 d) gezeigt: In den (011) Ebenen ist eine Druck- und Zugspannung $\pm \epsilon$ abwechselnd zu finden. Nach außen ist die $4 \times 4$ Überstruktur voll kompensiert. Offensichtlich führt diese Art der Verspannung zu der in Abb.23 a) und b) gezeigten Erhöhung der Übergangstemperatur. Aus diesem Ergebnis lässt sich eine Multilagen-Regel formulieren, die zu der Kationen-Ordnung bzw. dem dargestellten Verspannungsprofil führt:

$$
\left[\left(p \operatorname{ReMnO}_{3}\right) /\left(\operatorname{ReAMn}_{2} \mathrm{O}_{6}\right)\right]_{m}
$$

wobei $p=1,2,3 \ldots$ für Manganate mit den entsprechenden $e_{g}$ Loch-Dotierungen $x=0.33,0.25,0.2, \ldots$ einzuhalten ist.

Ebenso sollte diese Regel für Manganate, mit einer höheren Übergangstemperatur und einer ausgeprägteren A-Platz Ionen Größendifferenz im Vergleich zum $L a_{0.75} C a_{0.25} M n O_{3}$, gelten. Für solche Materialien, wie z.B. $L a_{0.67} \mathrm{Sr}_{0.33} \mathrm{MnO}_{3}$ oder $L a_{0.67} B a_{0.33} \mathrm{MnO}_{3}$, ist es vorstellbar, die bisher geltende maximal observierte Übergangstemperatur von $370 K$ zu übertreffen, was für spintronische Anwendungen bei Raumtemperatur notwendig ist.

Innerhalb dieser Arbeit sollte ergründet werden, wie sich eine künstliche Kationen Ordnung, die mittels eines Multilagen-Ansatzes realisiert wurde, auf das $\mathrm{La}_{0.67} \mathrm{Sr}_{0.33} \mathrm{MnO}_{3}$ auswirkt. An einer anderen Stelle des Phasendiagramms von LSMO, explizit für $\mathrm{La}_{1 / 3} \mathrm{Sr}_{2 / 3} \mathrm{MnO}_{3}$, konnte bereits gezeigt werden, dass eine Erhöhung der Néel Temperatur von $\sim 70 \mathrm{~K}$ durch eine künstliche Multilagen-Kationen-Ordnung mit $\left[\left(\mathrm{LaMnO}_{3}\right)_{n} /\left(\mathrm{SrMnO}_{3}\right)_{2 n}\right]_{m}$ auf STO(001) realisiert werden konnte [9]. Neben der angesprochenen Regel $\left[\left(\mathrm{LaMnO}_{3}\right)_{n} /\right.$ $\left.\left(\mathrm{La}_{0.5} \mathrm{Sr}_{0.5} \mathrm{MnO}_{3}\right)_{2 n}\right]_{m}$ (LS/LMO) wurde auch der Ansatz [( $\left.\left.\mathrm{LaMnO}_{3}\right)_{2 n} /(\mathrm{SrMnO})_{n}\right]_{m}$ (L/SMO) untersucht. Die Multilagen-Systeme wurden über die Multilagen-Regel hinausgehend auf größere Bilagen-Dicken, über den Index $n$ mit der Wiederholung $m$, ausgedehnt. Wie im nächsten Teilkapitel gezeigt wird, kann das $\mathrm{La}_{1-x} \mathrm{Sr}_{x} M n \mathrm{O}_{3}$ System, aufgrund eines stepflow-Wachstums, sehr glatt auf $\mathrm{SrTiO}_{3}(001)$ (STO) Substraten gewachsen werden. Diese glatten Oberflächen werden benötigt, um scharfe Grenzflächen in dem Multilagen-Systemen zu erhalten, welche wiederum notwendig sind, damit der gewünschte Grad an KationenOrdnung erreicht wird. Begründet durch diese Tatsache wurde STO als Substrat für diese Serie gewählt. Ein Nachteil dieses Substratmaterials ist, dass das LSMO System durch die 
4 A-Platz Ordnung in ferromagnetischen halbmetallischen Manganaten

strukturelle Fehlanpassung zum Substrat eine Druckspannung erfährt, die zu einer Reduktion der Übergangstemperatur führt [16-18, 29], die dem angestrebten Ziel, der Erhöhung dieser Temperatur, entgegen wirkt. Da die Druckspannung und damit die Übergangstemperatur von der Orientierung der Substratoberfläche abhängt [104], wurde das LS/LMO und das L/SMO System sowohl auf STO(001) als auch auf STO(111) präpariert und untersucht.

\subsubsection{Präparation von $\mathrm{La}_{1-\mathrm{x}} \mathrm{Sr}_{\mathrm{x}} \mathrm{MnO}_{3}$ mit MAD}

In diesem Teilkapitel sollen zunächst die Präparation und die Eigenschaften von dünnen $\mathrm{La}_{1-x} \mathrm{Sr}_{x} \mathrm{MnO}_{3}$ Schichten vorgestellt werden. Wie bereits erwähnt, ist das $\mathrm{LaMnO}_{3}$ (x=0, LMO) als Bulk-Kristall ein A-Typ AFM Isolator [67, 68]. Als dünner Film auf STO(001) ist das gleiche Material, aufgrund der durch das Substrat induzierten Verspannung, ein ferromagnetischer Isolator mit einer Curie Temperatur von $T_{C} \sim 150 K$ [105-107]. Auf diesem Substrat kann eine hohe $L a$-Leerstellenkonzentration in die LMO Struktur induziert werden, welche äquivalent zu einer Dotierung mit Erdalkalimetallen ist. Entsprechend besitzt das La-defizitäre LMO einen PMI-FMM Phasenübergang, wobei die Übergangstemperatur und die elektrische Leitfähigkeit direkt von der Leerstellenkonzentration abhängen [108]. Diese Eigenschaft kann ausgenutzt werden, um das stöchiometrische $L a / M n$-Verhältnis zu bestimmen. Entsprechend wurde das optimale $L a / M n$-Verhältnis ermittelt, indem in einer Probenserie das angesprochene Verhältnis variiert wurde. Zeigt der temperaturabhängige, elektrische Widerstand eines LMO Filmes keine Andeutung eines MIT und eine atomar glatte Oberfläche, ist das optimale Verhältnis gefunden. Der temperaturabhängige Widerstand für 26nm dicke LMO Filme mit optimalen $L a / M n$-Verhältnis sowie mit einem um $-6.4 \%$ vom optimalen Wert reduzierten $L a / M n$-Verhältnis (La-defizitär) ist in Abb.24 a) dargestellt. Es ist deutlich ein Knick im temperaturabhängigen Widerstand des La-defizitären LMO Films zu sehen, der einen MIT andeutet. Wie bereits aus der Literatur bekannt, zeigt der LMO Film auf STO(001) ein FM Verhalten ab einer Temperatur von $T_{C} \sim 175 K$. Das Sättigungsmoment von $4 \mu_{B} / M n$ bei tiefen Temperaturen und hohen Feldern, entspricht der maximalen zu erwartenden Magnetisierung für ein ferromagnetisches $M n^{3+}$ System. Die entsprechenden Messungen sind in Abb.24 b) dargestellt. In Abb.25 b) sind STM Aufnahmen von LMO Filmen mit verschiedenen $L a / M n$-Verhältnissen dargestellt. Das Bild des Films mit einem optimalen $L a / M n$-Verhältnis zeigt eine atomar glatte Oberfläche, die Stufen als Ebenbild der STO Substratoberfläche sind klar zu erkennen. Die Filme mit erhöhten oder reduzierten stöchiometrischen Verhältnissen zeigen deutlich Oberflächenrauigkeiten. Die XRD Messung von optimal stöchiometrischen LMO ist in Abb.26 a) gezeigt. Der out-of-plane Gitterparameter lässt sich, aufgrund der Ähnlichkeit zum Gitterparameter des Substrates, 

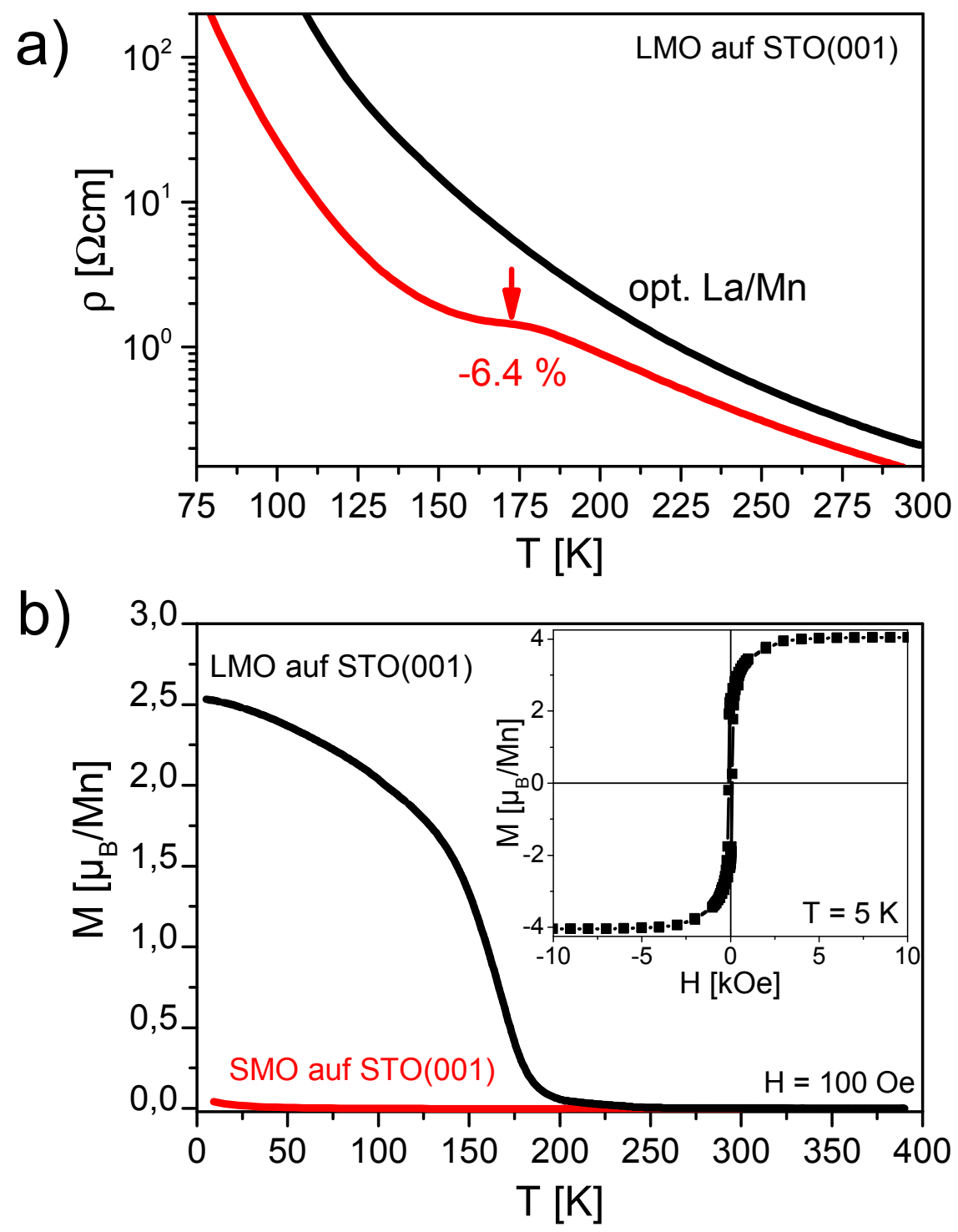

Abbildung 24: a) Temperaturabhängigkeit des spez. Widerstandes $\rho(T)$ von LMO Dünnfilmen auf STO(001) Substraten mit einem optimalen La/Mn-Verhältnis (schwarz) und einem um $6.4 \%$ reduzierten La/Mn-Verhältnis (rot, weniger La) und einer Schichtdicke von 26nm. Die Andeutung eines MIT ist mit einem roten Pfeil markiert. b) Abkühl-Magnetisierungskurve eines LMO (schwarz) und eines SMO (rot) Filmes mit optimalen stöchiometrischen Verhältnissen auf STO(001) bei einem externen Feld von 100Oe. Das eingebettete Diagramm zeigt die $M(H)$ Hysterese-Schleife für den LMO Film bei einer Temperatur von $5 K$. 

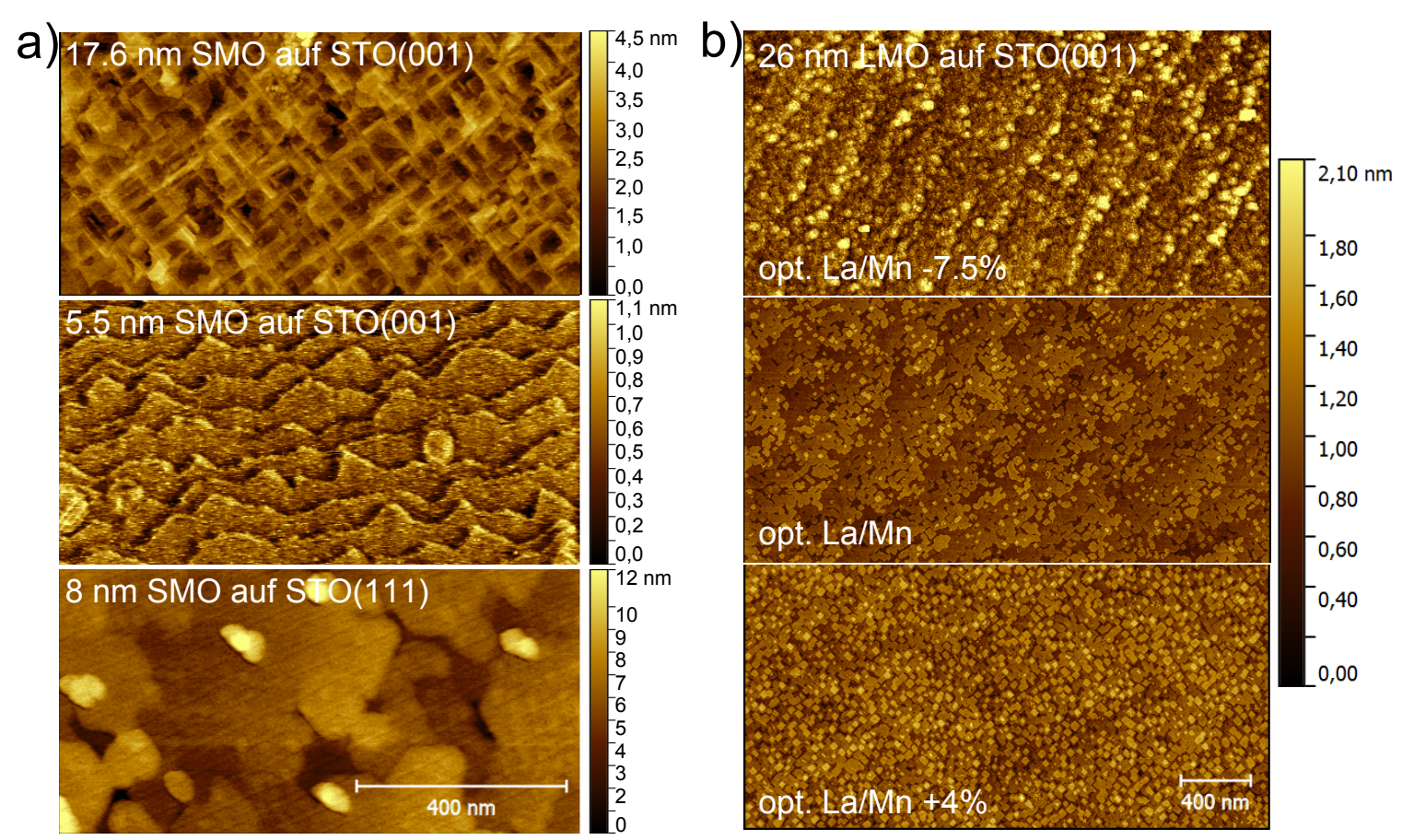

Abbildung 25: STM Aufnahme von a) SMO Filmen mit optimalem Sr/Mn-Verhältnis auf STO(001) mit einer Schichtdicke von $17.6 \mathrm{~nm}$ und 5.5nm und auf STO(111) mit einer Schichtdicke von 8nm; b) LMO Filmen mit einer Schichtdicke von $~ 26 \mathrm{~nm}$ auf STO(001) mit optimalem, um $-7.5 \%$ reduziertem und um $+4 \%$ erhöhtem La/Mn-Verhältnis.

nur schwer bestimmen. Wegen der hohen kristallinen Qualität des LMO Films, sind LaueOszillationen vorhanden, mit denen, wie in dem eingebetteten Diagramm in Abb.26 a) angedeutet, die out-of-plane Gitterkonstante auf $c=0.386 \mathrm{~nm}$ abgeschätzt werden kann. Im Vergleich mit Werten aus der Literatur mit $c>0.39 \mathrm{~nm}$ für LMO Filme auf STO(001) Substrat $[109,110]$ ist dieser Wert zu klein. Diese Differenz lässt sich jedoch auf die Unterschiede in den Depositionsmethoden in der Literatur (PLD und MBE) und dieser Arbeit (MAD) zurück führen. MAD funktioniert in der Umgebungsatmosphäre mit einem Sauerstoffpartialdruck von $\sim 200 \mathrm{mBar}$, wodurch man davon ausgehen kann, das nahezu keine Sauerstoffleerstellen im Film vorhanden sind. Zhao et al. konnte mit einer LMO Film Probenserie auf STO(001), die mit einer Laser-MBE-Methode bei verschiedenen Sauerstoffpartialdrücken präpariert wurde zeigen, dass der out-of-plane Gitterparameter mit steigendem Sauerstoffgehalt, bis auf einen Minimalwert von $0.389 \mathrm{~nm}$, der mit dieser Methode erreicht werden konnte, abnimmt [109].

Das $\mathrm{SrMnO}_{3}$ (x=1, SMO) ist hingegen, aufgrund der fehlenden $e_{g}$ Elektronen, ein G-Typ AFM Isolator [68]. In diesem System existieren keine signifikanten Änderungen der magnetischen oder elektrischen Eigenschaften als Funktion der Stöchiometrie, wie in LMO. Für ein $S r / M n$-Verhältnis nahe dem La/Mn-Verhältnis, können strukturelle Eigenschaften ge- 


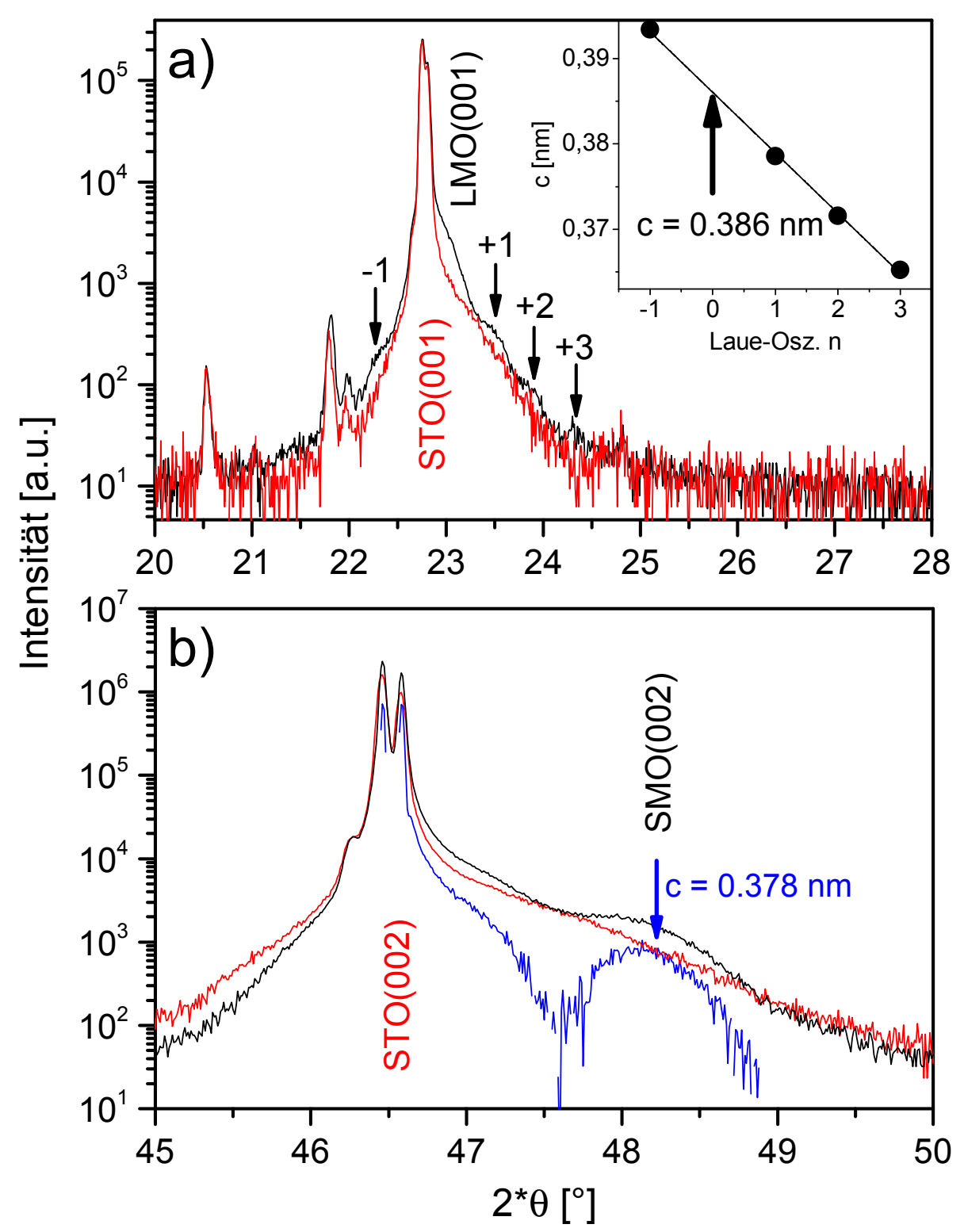

Abbildung 26: a) XRD Messung eines LMO Filmes auf STO(001) mit einer Schichtdicke von $26 \mathrm{~nm}$ (schwarz). Die out-of-plane Gitterkonstante, die nahe der des Substrates ist, lässt sich mit Hilfe der Laue-Oszillationen (markiert mit Pfeilen) auf eine Größe von c $=0.386 \mathrm{~nm}$ festlegen (siehe eingebettetes Diagramm). b) XRD Messung eines SMO Filmes auf STO(001) mit einer Schichtdicke von 18nm (schwarz). Nach dem Abzug des Substrates (Resultat in blau) ist der deutlich verbreitete (002) Reflex des SMO Filmes deutlich zu erkennen. Die mittlere out-of-plane Gitterkonstante beträgt $c=0.378 n m$. Die Leersubstrat-Messung ist in beiden Diagrammen in rot gezeigt. 
4 A-Platz Ordnung in ferromagnetischen halbmetallischen Manganaten

messen werden, die den Erwartungen entsprechen: Wie in Abb.25 a) zu sehen ist, erhält man für dünne Filme auf STO(001) (hier mit einer Schichtdicke von 5.5nm) eine atomar glatte Oberfläche, die, wie auch schon für LMO, auf ein step-flow-Wachstum der Schichten zurück schließen lässt. Auf STO(001) hingegen, können Stufen beobachtet werden, die wesentlich größer als eine Monolage des SMO Materials sind. Hier findet man eine Art stepbunch-Wachstum, das für LMO auf STO(111) nicht anzutreffen ist. Im folgenden wird ein Erklärungsansatz für diese Problematik vorgestellt: Während die $(\mathrm{SrO})$ und $\left(\mathrm{MnO}_{2}\right)$ Halblagen von SMO in der (001) Ebene elektrisch neutral sind, sind die entsprechenden $(M n)^{4+}$ und $\left(\mathrm{SrO}_{3}\right)^{4-}$ Halblagen in der (111) Ebene geladen. LMO ist in beiden betrachteten Ebenen elektrisch geladen, kann jedoch ein teilweises Fehlen einer Perowskit-Halblage durch das Einbringen von $\mathrm{Mn}^{4+}$ ausgleichen. Dieser Mechanismus scheint auf das SMO nicht übertragbar zu sein, da es möglicherweise energetisch ungünstig ist, das benötigte $M n^{5+}$ zu bilden. Daraus würde folgen, dass das SMO versucht die Ladung entsprechend auszugleichen, was zu dem beobachteten Wachstumsmodus führt. Für dickere SMO Filme (17.6nm) auf STO(001) raut der Film stark auf. Wie man in Abb.26 b) sehen kann, sind die XRD (001) Reflexe der dickeren SMO Filme (hier 18nm) verbreitert. Der mittlere out-of-plane Gitterparameter lässt sich nach dem Abzug der Substrat-Messung auf $c=0.378 \mathrm{~nm}$ bestimmen. In den L/SMO Übergittern kann später gezeigt werden, dass die out-of-plane Gitterkonstante für wenige Monolagen von SMO auf $c=0.368 \mathrm{~nm}$ bestimmt werden kann. All diese Beobachtungen lassen den Schluss zu, dass, aufgrund der großen Fehlanpassung zwischen SMO und STO $(\sim 2.6 \%)$, der stark verspannte SMO Film für Schichtdicken > 5nm entspannt. Dabei raut die Oberfläche auf und die Gitterkonstante nähert sich der Bulk-Gitterkonstante von $0.3805 \mathrm{~nm}[111]$ an.

Mit den ermittelten stöchiometrischen Faktoren kann das gesamte $\mathrm{La}_{1-x} \mathrm{Sr}_{x} \mathrm{MnO}_{3}$ (LSMO) System präpariert werden. In Abb.27 sind die magnetischen und elektrischen Eigenschaften von verschiedenen $37 \mathrm{~nm}$ dicken $\mathrm{La}_{0.67} \mathrm{Sr}_{0.33} \mathrm{MnO}_{3}$ Filmen dargestellt. Die Filme unterscheiden sich im verwendeten Substrat und in der Depositionmode: Während der LSMO Film auf STO(001), wegen der Substrat-induzierten Verspannung, eine Übergangstemperatur von $352 K$ zeigt, liegt die Übergangstemperatur für einen LSMO Film auf STO(111) bei 358K. Beide Filme wurden kontinuierlich mit einer konstanten Rate von $6.5 \mathrm{~nm} / \mathrm{min}$ deponiert. Ein dritter Film wurde, ähnlich wie die Übergitter weiter unten, pulsiert auf STO(111) deponiert, d.h. es wurden vier Monolagen Film mit einer konstanten Rate von $6.5 \mathrm{~nm} / \mathrm{min}$ deponiert und nach einer Wartezeit von vier Sekunden wurden weitere vier Monolagen gewachsen. Dies wurde bis zur endgültigen Schichtdicke von $37 \mathrm{~nm}$ fortgeführt. Dieser Film zeigt eine noch höhere Übergangstemperatur von $363 K$, die bemerkenswerterweise größer ist, als die höchste in der Literatur berichtete Curie-Temperatur für LSMO Dünnfilme, die $360 K$ beträgt 


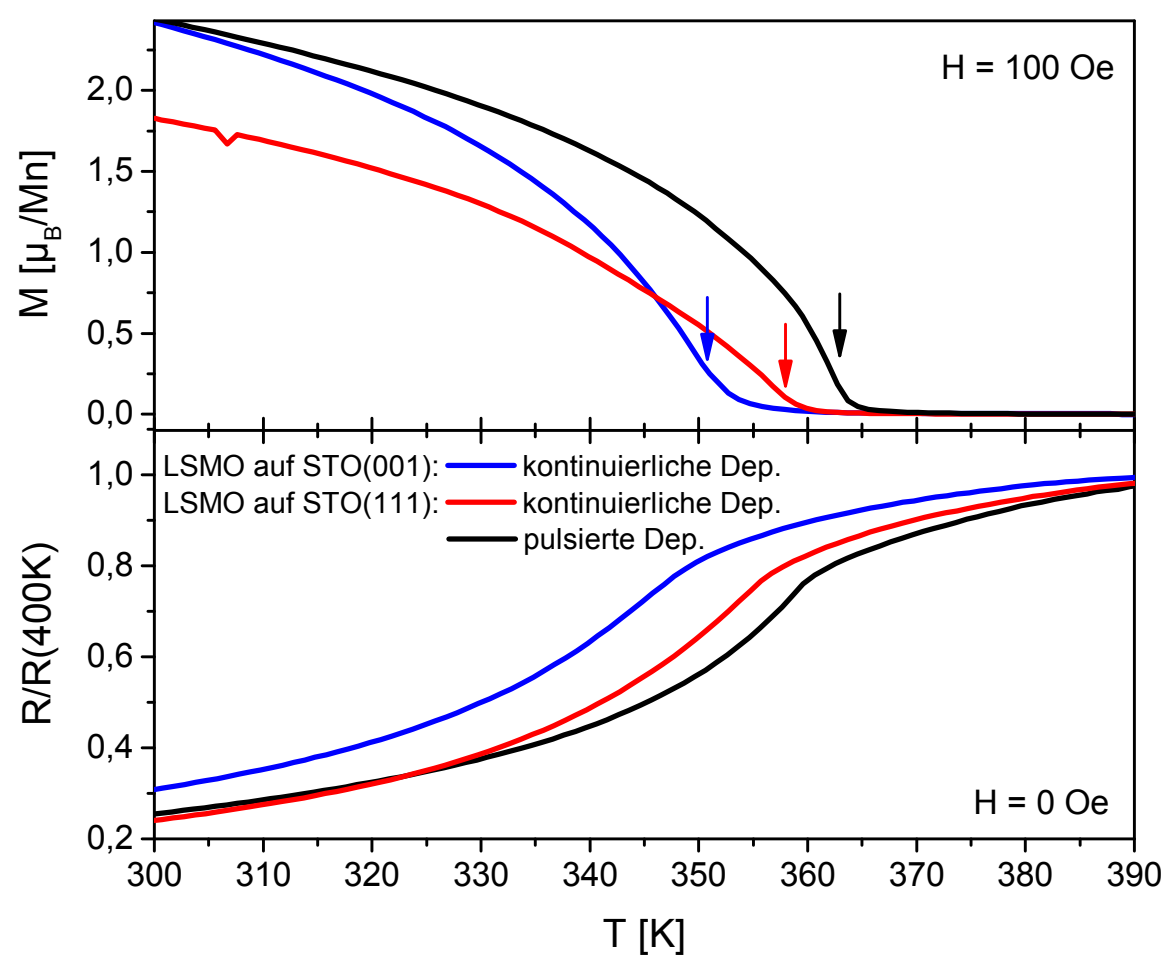

Abbildung 27: Die Abkühl M(T)-Kurven bei einem externen Feld von 100Oe und der temperaturabhängige elektrische Widerstand $R(T)$ bei Nullfeld, normiert auf $R(400 K)$, für verschiedene $\mathrm{La}_{0.67} \mathrm{Sr}_{0.33} \mathrm{MnO}_{3}$ Filme: kontinuierlich deponiertes LSMO auf STO(001) (blau), auf STO(111) (rot) und pulsiert deponiertes LSMO auf STO(111) (schwarz). Die Übergangstemperaturen sind mit Pfeilen markiert.

[113]. Alle aufgeführten Filme zeigen einen Metall-Metall Übergang an den entsprechenden Übergangstemperaturen und eine volle Sättigungsmagnetisierung von $\sim 3.7 \mu_{B} / M n$.

Der Verlauf der Curie-Temperatur für kontinuierlich deponierte $\mathrm{La}_{1-x} \mathrm{Sr}_{x} M n \mathrm{O}_{3}$ Filme auf STO(001) (LSMO(001)) und STO(111) (LSMO(111)) als Funktion der Sr-Dotierung $x$, ist von Danny Schwarzbach im Rahmen seiner Masterarbeit untersucht worden und ist in Abb.28 dargestellt. Während die größte Übergangstemperatur von $\operatorname{LSMO}(001)$ bei einer Dotierung von $x=0.3$ angetroffen wird, ist sie für $\operatorname{LSMO}(111)$ bei $x=0.4 \mathrm{zu}$ finden. Allgemein hat der Verlauf der Funktion $T_{C}(x)$ für LSMO(111) Filme eine große Ähnlichkeit mit der $T_{C}(x)$ Funktion für $L a_{1-x} S r_{x} M n O_{3}$ Bulk-Einkristalle [114]. Die $T_{C}(x)$ Kurve ist für LSMO(001) zu kleineren Werten von $x$ verschoben und die maximale observierte Übergangstemperatur ist kleiner als die des LSMO(111). Die out-of-plane Gitterkonstante von LSMO(001) mit $x=0.33$ beträgt $0.385 \mathrm{~nm}$, sie unterliegt somit einer Zugverspannung durch das Substrat: Bei dieser Verspannung wird ein Gitterparameter reduziert, während die anderen zwei innerhalb der (001)-Ebene vergrößert sind. Mit dieser Verspannung ist die niedrigere Übergangstemperatur 


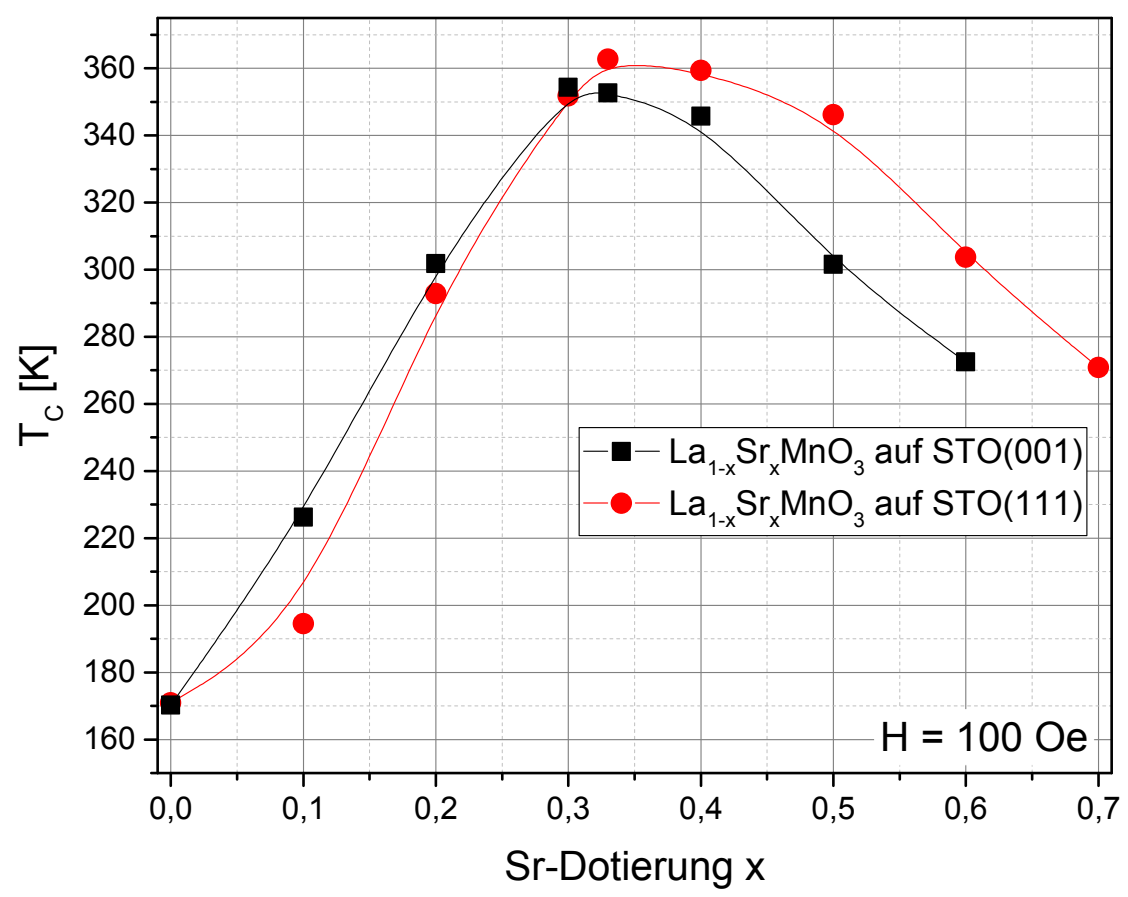

Abbildung 28: Die Curie-Temperatur als Funktion der $\mathrm{Sr}$-Dotierung für das $\mathrm{La}_{1-x} \mathrm{Sr}_{x} \mathrm{MnO} \mathrm{O}_{3}$ System auf STO(001) und STO(111). Die Linien dienen als optische Führung. Die Daten sind aus [112] entnommen.

für LSMO(001) im Vergleich zu LSMO(111) oder LSMO Einkristallen über die präferierte Besetzung des $d_{x^{2}-y^{2}}$-Orbitals $[16-18,29]$ zu verstehen (siehe Kap. 2.3). Bei LSMO(111) sind alle kubischen Achsen gleichmäßig verspannt und es liegt keine präferierte Elektronenbesetzung eines $e_{g}$ Orbitals vor. Dies äußert sich durch die Abwesenheit der magnetokristallinen Anisotropie [104] und des Bulk-ähnlichen $T_{C}(x)$ Verlaufs. Entsprechend ist auch die Übergangstemperatur höher als in $\operatorname{LSMO}(001)$.

Um im Weiteren die LSMO Übergitter mit der genauen Bilagen-Dicke wachsen zu können, wurde zunächst eine Schichtdickenserie von LMO, SMO und LSMO auf STO(001) präpariert. Die Schichtdicke $d$ sowie die RMS-Rauigkeiten der Oberfläche $\sigma_{s}$ und der Grenzfläche zwischen Film und Substrat $\sigma_{\text {Film } / \text { STO }}$ wurde anhand von Simulationen von XRR-Messungen ermittelt. Die Simulationen wurden mit der Software ReMagX durchgeführt [51]. In Abb.29

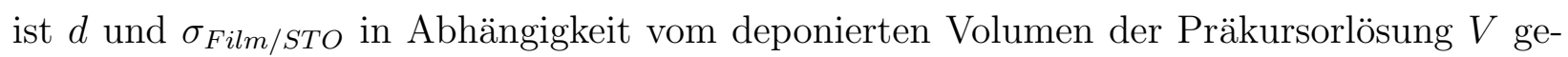
zeigt. Während sich das quadratische Mittel der Oberflächenrauigkeit unabhängig von der Schichtdicke zwischen $\sigma_{s} \sim 0.2-0.4 \mathrm{~nm}$ bewegt, steigt die Grenzflächenrauigkeit $\sigma_{\text {Film } / \text { STO }}$ monoton mit der Schichtdicke $d$ an. Hervorzuheben ist, dass die Grenzflächenrauigkeit für LMO besonders groß ist. Diese Beobachtung lässt sich mit der „polaren Katastrophe“ in Oxiden erklären, ein Modell, das ursprünglich als Erklärung für die hohe elektrische Leitfähigkeit 


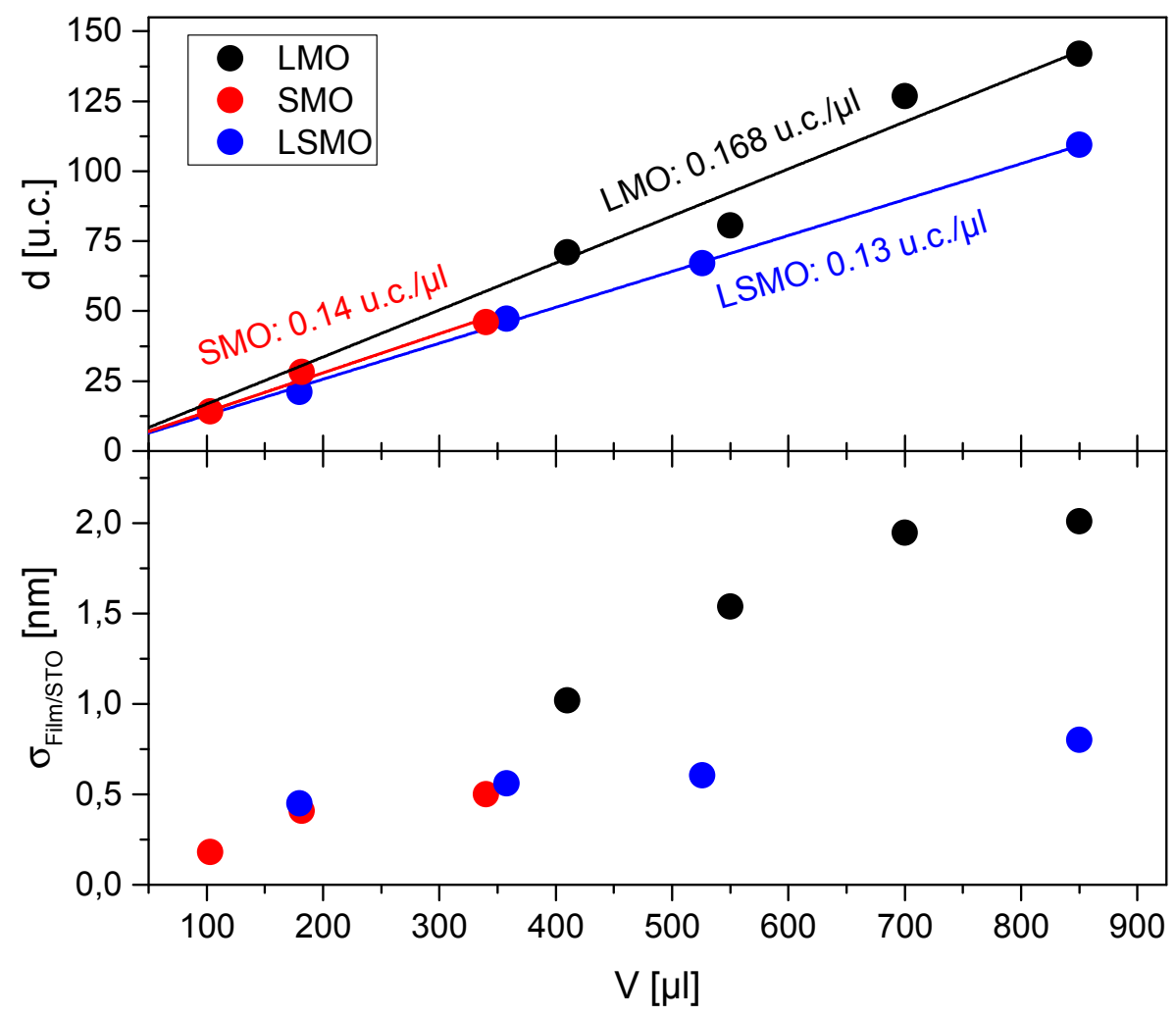

Abbildung 29: Die Schichtdicke d als Funktion des Volumens der deponierten Präkursorlösung V für LMO (schwarz), SMO (rot) und LSMO (blau) Filme auf STO(001) Substrat. Über eine lineare Anpassung erhält man die d/V-Verältnisse für die verschiedenen Materialien. Des Weiteren ist

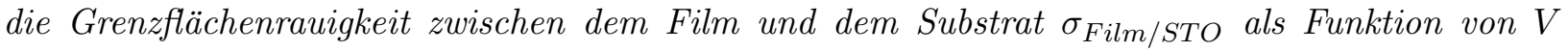
dargestellt.

an der $\mathrm{LaAlO}_{3} / \mathrm{SrTiO}_{3}$ Grenzfläche erdacht wurde [115]: Betrachtet man die PerowskitHalblagen, so sind die $\left(\mathrm{TiO}_{2}\right)^{0}$ und $(\mathrm{SrO})^{0}$ Halblagen elektrisch neutral, wobei die $\left(\mathrm{MnO}_{2}\right)^{-1}$ und $(\mathrm{LaO})^{+1}$ Halblagen elektrisch geladen sind. Mit der wachsenden LMO Schicht auf STO würde sich in diesem Modell das elektrische Potential immer weiter erhöhen. Hierunter versteht man die polare Katastrophe. Diese kann durch das Einbringen von Defekten, wie z.B. Sauerstoffleerstellen oder durch die Veränderung der Dotierung an der Grenzfläche mittels Diffusion, die zur Erhöhung der Grenzflächenrauigkeit führt, verhindert werden. Generell ist dieses Problem für SMO nicht vorhanden. In LSMO kann die polare Katastrophe, durch gezieltes Ändern der Manganvalenz an der Grenzfläche zu $\mathrm{Mn}^{+4}$, vermindert werden. Wie in Abb.29 zu sehen ist, kann die Schichtdicke für LMO, aufgrund der hohen Grenzflächenrauigkeit, nicht zuverlässig bestimmt werden. Zudem sollte das $d / V$-Verhältnis von LMO, wegen der Normierung der Schichtdicke auf die Größe einer pseudokubischen Einheitszelle u.c., mit dem $d / V$-Verhältnissen von SMO und LSMO, die nahezu gleich sind, übereinstimmen. Da die out-of-plane Gitterkonstante von SMO, wegen der großen Verspan- 
4 A-Platz Ordnung in ferromagnetischen halbmetallischen Manganaten

nung durch das Substrat, stark von der Schichtdicke abhängt, resultiert auch eine gewisse Unsicherheit in dem $d / V$-Verhältnis von SMO. Somit wurde für die im nächsten Kapitel vorgestellten Übergittersysteme das $d / V$-Verhältnis von LSMO verwendet. In dieser Pro-

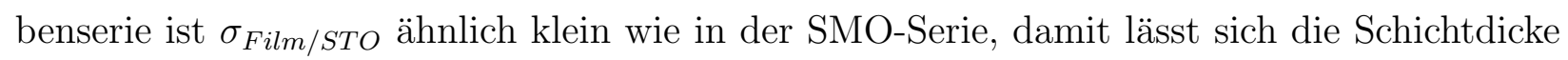
zuverlässig bestimmen. Zusätzlich ist der out-of-plane Gitterparameter in dem hier betrachteten Schichtdicken-Intervall kaum von der Schichtdicke abhängig.

\subsubsection{Strukturelle Eigenschaften der LSMO Übergitter}

Für diese Arbeit wurden $\left[\left(\mathrm{LaMnO}_{3}\right)_{n} /\left(\mathrm{La}_{0.5} \mathrm{Sr}_{0.5} \mathrm{MnO}_{3}\right)_{2 n}\right]_{m}$ (LS/LMO) als auch $\left[\left(\mathrm{LaMnO}_{3}\right)_{2 n} /\left(\mathrm{SrMnO}_{3}\right)_{n}\right]_{m}$ Übergitter mit $n=1 \ldots 6$ und $m=9 \ldots 50$ mit der MAD-3 Anlage präpariert. Dabei wurde $m$ möglichst so gewählt, dass alle Filme die gleiche Gesamtschichtdicke $d \sim 37 \mathrm{~nm}$ besitzen. Diese Übergitter stellen eine A-Platz (teil-)geordnete Variante des $\mathrm{La}_{0.67} \mathrm{Sr}_{0.33} \mathrm{MnO}_{3}$ dar. In diesem Teilabschnitt sollen exemplarisch die strukturellen Eigenschaften dieser Heterostrukturen vorgestellt werden.

Die folgenden HAADF und EELS TEM Messungen an diesen Übergittern wurden von der Gruppe um J. Verbeeck am EMAT der Universität von Antwerpen und von V. Roddatis aus dem Institut der Materialphysik der Georg-August-Universität Göttingen durchgeführt und aufbereitet. Aus allen TEM Messungen ist erkennbar, dass die betrachteten Übergitter epitaktisch in einer hohen kristallinen Qualität auf dem Substrat aufgewachsen sind. In Abb.30 ist eine HAADF TEM Aufnahme von a) L/SMO mit $n=6$ und b) LS/LMO mit $n=4$ auf STO(111) dargestellt. In den Bildern sind die SMO (in a) bzw. $\mathrm{La}_{0.5} \mathrm{Sr}_{0.5} \mathrm{MnO}_{3}$ (LSMO:0.5, in b) von den LMO Lagen klar zu unterscheiden. Wegen der exponentiellen Abhängigkeit der HAADF TEM Intensität von der atomaren Ordnungszahl $Z$, sind die $S r$ beinhaltenden Lagen dunkler als die LMO Lagen $\left(Z_{L a}=57 \mathrm{zu} Z_{S r}=38\right)$. In beiden Bildern ist eine große Grenzflächenrauigkeit zwischen den verschiedenen Materialien der Heterostruktur zu erkennen. Bemerkenswerterweise ist in beiden Abbildungen die Dicke der Lagen, die $S r$ enthalten zu dünn, z.B. sollte das L/SMO $n=6$ Übergitter pro Bilage 6u.c. SMO und 12u.c. LMO enthalten, in Abb.30 a) können jedoch durchschnittlich 3u.c. SMO und 15u.c. LMO gezählt werden. Entsprechend verhält es sich auch in Abb.30 b), in der das LMO 3u.c. zu dick ist. Zudem ist in der hier dargestellten Überstruktur die Bilagen-Dicke leicht zu groß. In Abb.31 ist die HAADF und EELS TEM Messung des L/SMO $n=3$ Übergitters auf STO(001) dargestellt. Aus den EELS Messungen konnten die elementspezifischen Karten für Ti, O, Mn und La gewonnen werden. Diese Karten bestätigen die Verteilung der APlatz Ionen aus der HAADF Messung. Die starke Durchmischung mit dem Substrat und die 


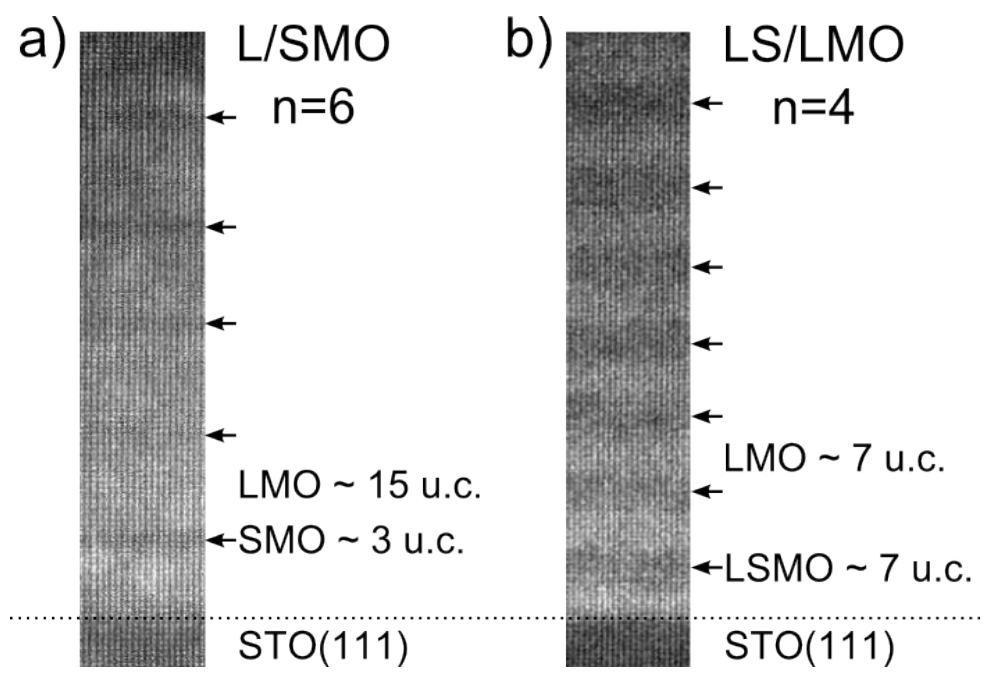

Abbildung 30: HAADF TEM Aufnahmen des a)L/SMO $n=6$ und b)LS/LMO $n=4$ Übergitters auf STO(111). Die Pfeile markieren die Lagen des SMO bzw. des LSMO:0.5 (dunklere Lagen). Die durchschnittlichen Schichtdicken der einzelnen Lagen sind angegeben.

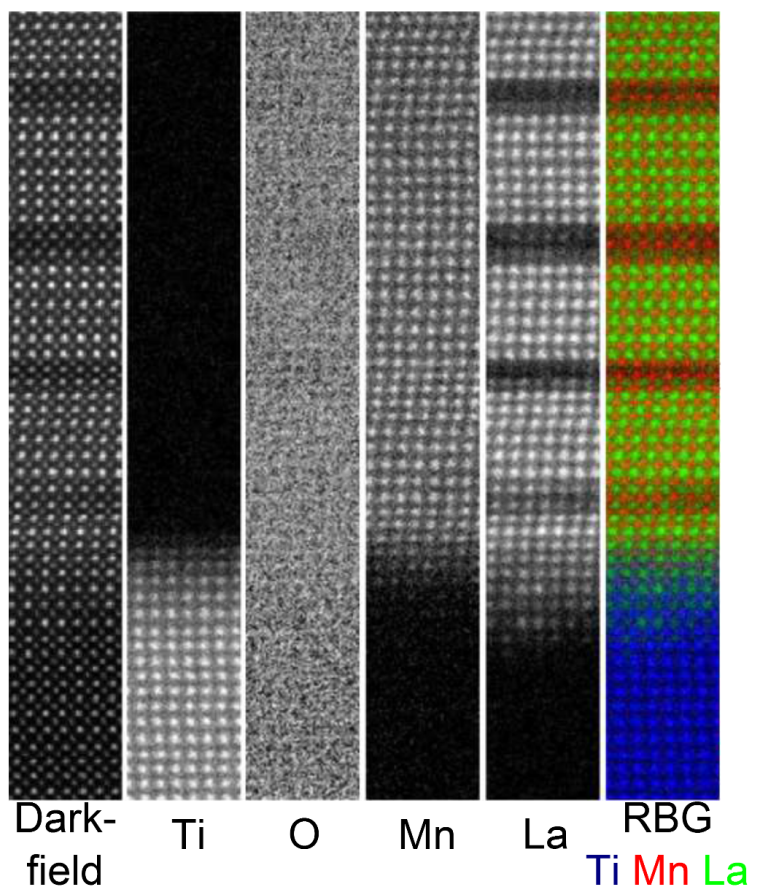

Abbildung 31: Dargestellt ist eine HAADF TEM Aufnahme (Darkfield) und TEM EELS elementspezifische Karten von Ti, O, Mn und La für das L/SMO Übergitter mit $n=3$ auf STO(001). Das rechte Bild zeigt eine Superposition der Ti (blau), Mn (rot) und La (grün) Karte. 

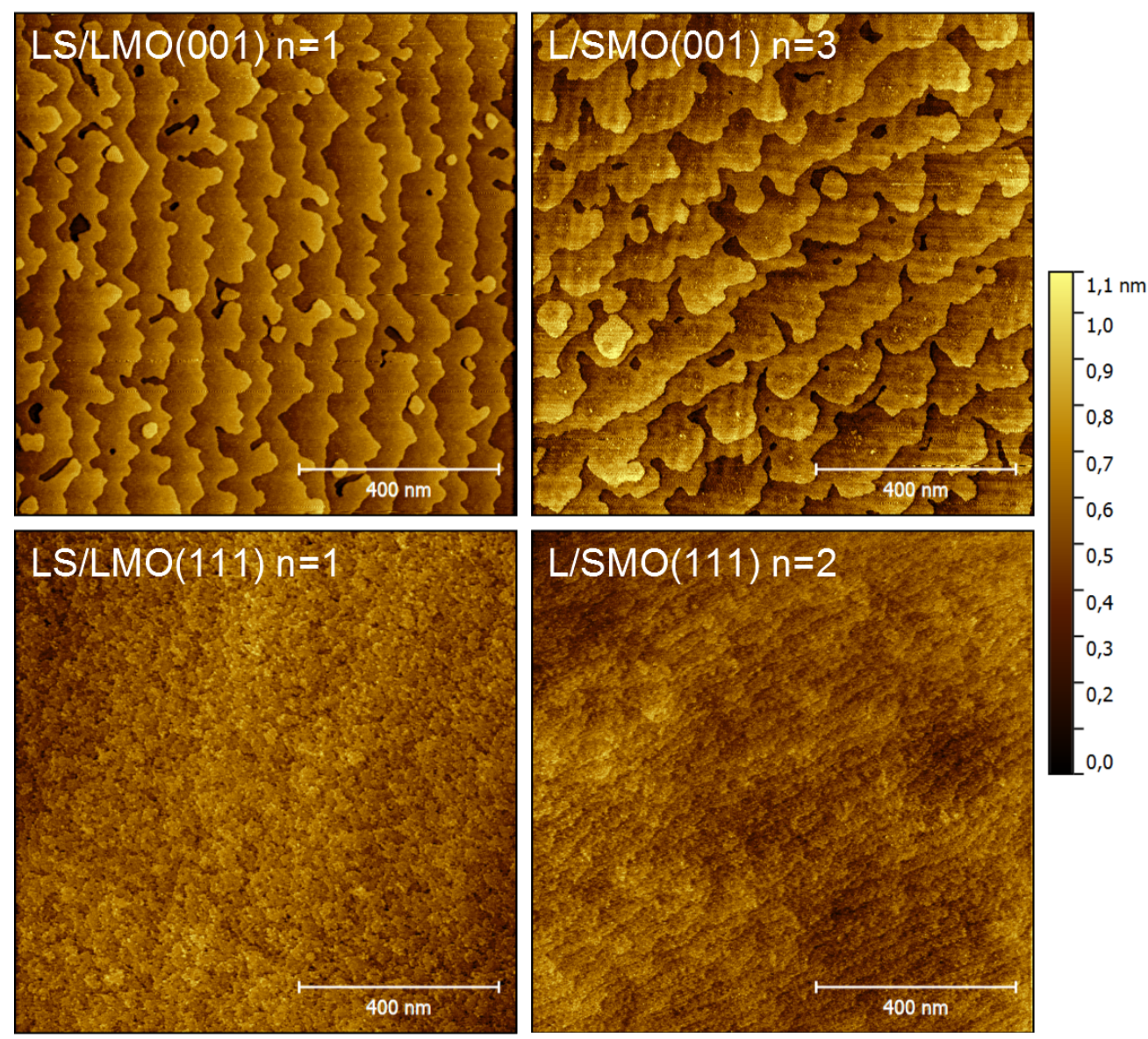

Abbildung 32: $1 \times 1 \mu m$ STM Aufnahmen des $L S / L M O(001)$ mit $n=1, L / S M O(001)$ mit $n=3$, $L S / L M O(111)$ mit $n=1$ und $L / S M O(111)$ mit $n=2$. Alle Bilder sind auf eine Größenskala normiert.

scharfen Grenzflächen zwischen SMO und LMO sind deutlich zu erkennen, wobei die erste SMO Lage stärker mit La durchmischt ist, als die Folgenden. Letzteres ist möglicherweise die Folge aus der rauen Grenzfläche zum Substrat. Erstaunlicherweise scheint die Grenzfläche, bei der LMO auf SMO folgt (in Wachstumsrichtung), schärfer zu sein als die Grenzfläche, bei der SMO auf LMO folgt. Die Sauerstoffkonzentration ist, wie schon erwartet, gleichmäßig und auf einem hohen Niveau. Es kann weiter davon ausgegangen werden, dass nahezu keine Sauerstoffleerstellen vorhanden sind.

Die STM Aufnahmen zeigen in allen hier vorgestellten Übergittern eine atomar glatte Oberfläche. In Abb.32 sind die Oberflächen für jeden Übergittertyp anhand eines Beispiels dargestellt. In allen Bildern sind deutlich die Stufen, die aus der Vizinalität des Substrates resultieren, zu erkennen. Seltener sind auch 2D-Inseln zu sehen. In den STM-Aufnahmen der (111)-Übergitter ist zu beachten, dass die Monolagen-großen Stufen um den Faktor $1 / \sqrt{3}$ kleiner sind als die Stufen der (001)-Übergitter. Dieser Faktor kommt folgendermaßen zustande: Die Raumdiagonale $a$ der vereinfachten pseudokubischen Kristallstruktur der Perowskite 


\begin{tabular}{|c|c|c||c|c|c|c|c|c|} 
Typ & $n$ & $m$ & BLT & $d_{2 n}$ & $d_{n}$ & $\sigma_{s}$ & $\sigma_{i}$ & $\sigma_{o}$ \\
\hline \hline \multirow{3}{*}{$\operatorname{LS} / \operatorname{LMO}(001)$} & 1 & 32 & 3.1 & 2.0 & 1.0 & 0.6 & 0.3 & 0.3 \\
\cline { 2 - 9 } & 2 & 16 & 6.0 & 3.9 & 2.1 & 0.6 & 0.6 & 0.3 \\
\cline { 2 - 9 } & 3 & 11 & 7.8 & 4.9 & 2.9 & 0.6 & 0.6 & 0.45 \\
\hline \hline \multirow{3}{*}{ L/SMO(001) } & 1 & 32 & 3.0 & 2.5 & 0.5 & 0.6 & 0.25 & 0.4 \\
\cline { 2 - 9 } & 2 & 16 & 5.2 & 4.1 & 1.1 & 0.6 & 0.2 & 0.45 \\
\cline { 2 - 9 } & 3 & 11 & 8.6 & 6.7 & 1.9 & 0.6 & 0.0 & 0.3 \\
\hline \hline \multirow{3}{*}{$\operatorname{LS} / \mathrm{LMO}(111)$} & 1 & 28 & 3.3 & - & - & - & - & - \\
\cline { 2 - 9 } & 2 & 28 & 5.9 & - & - & 0.6 & 0.37 & 0.4 \\
\cline { 2 - 9 } & 4 & 14 & 11.9 & 7.9 & 4.0 & 0.6 & 0.4 & 0.3 \\
\hline \hline \multirow{3}{*}{$\mathrm{L} / \mathrm{SMO}(111)$} & 1 & 50 & 3.2 & - & - & - & - & - \\
\cline { 2 - 9 } & 2 & 28 & 5.7 & - & - & - & - & - \\
\cline { 2 - 8 } & 4 & 14 & 11.8 & - & - & - & - & - \\
\cline { 2 - 8 } & 6 & 9 & 17.1 & - & - & - & - & - \\
\hline \hline
\end{tabular}

Tabelle 1: Strukturelle Daten aller hier behandelten LSMO Überstrukturen. Die Bilagendicke, BLT, die Schichtdicke der 2n-Komponente (LMO oder LSMO:0.5), $d_{2 n}$ und die Schichtdicke der $n$-Komponente (SMO oder LMO), $d_{n}$ ist in Monolagen u.c. angegeben. Es muss beachtet werden, das die (111)-Monolage (001)-Monolage / $\sqrt{3}$ ist. Die quadratischen Mittel der Rauigkeiten an der Film/Substrat Grenzfläche $\sigma_{s}$, an den Filmgrenzflächen $\sigma_{i}$ und an der Filmoberfläche $\sigma_{o}$ sind in $n m$ angegeben.

mit dem Gitterparameter $c_{(001)}$ ist $a=\sqrt{3} c_{(001)}$. Auf der Länge $a$ schneidet diese Diagonale sechs (111)-Flächen: drei mit A-Platz Ionen und drei mit B-Platz Ionen. Der Abstand zwischen zwei $A B O_{3}$ Ebenen in (111) Richtung ist demnach $c_{(111)}=\sqrt{3} / 3 c_{(001)}=c_{(001)} / \sqrt{3}$.

Da die TEM Untersuchung, aufgrund der Präparation der TEM-Lamelle, der Messung selbst und der Aufbereitung der Daten, sehr zeit- und ressourcenintensiv ist, wurde diese anhand den bereits gezeigten drei Proben dieser Probenserie durchgeführt. Für die strukturelle Charakterisierung aller Übergitter wurde XRR genutzt. Exemplarisch sind die XRR Messungen für jeden Übergittertyp mit näherungsweise gleicher Bilagen-Dicke ${ }^{15}$ in Abb.33 aufgetragen. Diese Messungen wurden mit Hilfe der Software ReMagX [51] simuliert und angepasst. Dazu wurde ein Übergittermodell erstellt, das die strukturellen Eigenschaften, die in den TEM und STM beobachtet wurden, beinhaltet: Die Substrat-Grenzfläche, als auch die Grenzflächen innerhalb der ersten Bilage, deren Rauigkeit mit der Substrat-Grenzflächenrauigkeit gleichgesetzt wurde, sind erhöht. Die Oberflächenrauigkeit wurde der Größenordnung, der in den STM-Messungen beobachteten Oberflächenrauigkeit, angepasst. Alle anderen Grenzflächen als auch Schichtdicken der 2n-Lage (LMO in L/SMO bzw. LSMO:0.5 in LS/LMO Übergittern) und der $n$-Lage (SMO in L/SMO bzw. LMO in LS/LMO Übergittern) wurden

\footnotetext{
${ }^{15}$ In diesem Fall: $n=2$ für Übergitter auf $\mathrm{STO}(001)$ und $n=4$ für Übergitter auf $\mathrm{STO}(111)$. Der Umrechnungsfaktor von $\sqrt{3}$ ist nahe dem hier veranschlagten Faktor von 2.
} 
4 A-Platz Ordnung in ferromagnetischen halbmetallischen Manganaten

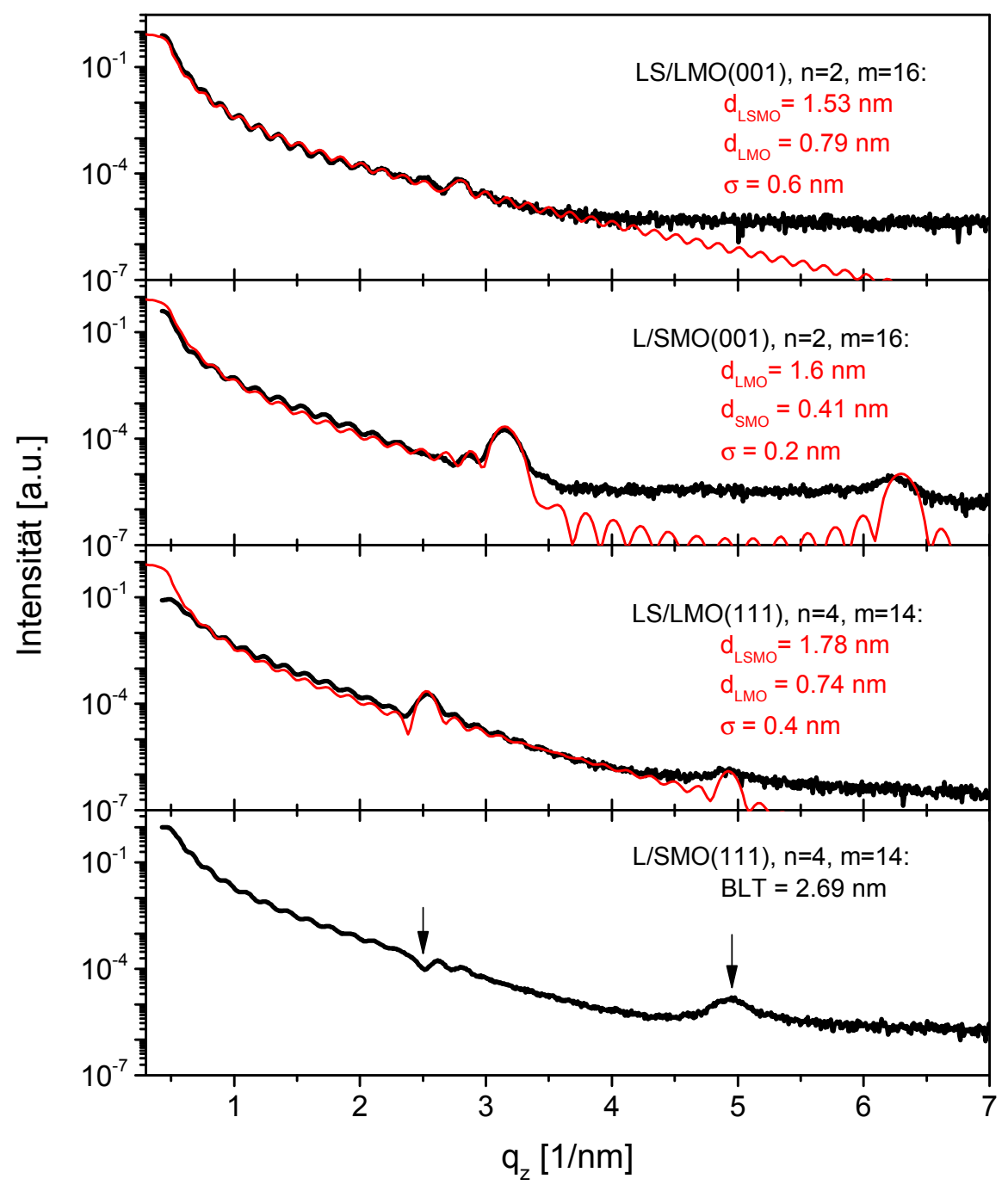

Abbildung 33: XRR Messungen (schwarz) der LS/LMO und L/SMO Übergitter mit $n=2, m=$ 16 auf $S T O(001)$ und mit $n=4, m=16$ auf $S T O(111)$. XRR Simulationen (rot) wurden mit der Software ReMagX [51] angefertigt. Die Simulationsparameter $d_{x M O}$ (Dicke der Schicht xMO) und $\sigma$ (quadratisches Mittel (RMS) der Grenzflächenrauigkeit) sind in rot angegeben. Die nicht angegebene (RMS) Oberflächenrauigkeit liegt für alle Filme zwischen $0.3-0.4 n m$. Es konnte kein Übergittermodell gefunden werden, sodass die XRR Simulation der XRR Messung des L/SMO(111) Übergitters mit $n=4$ entspricht. Die Bilagen-Dicke (BLT) dieses Übergitters konnte auf $2.69 \mathrm{~nm}$ bestimmt werden. 


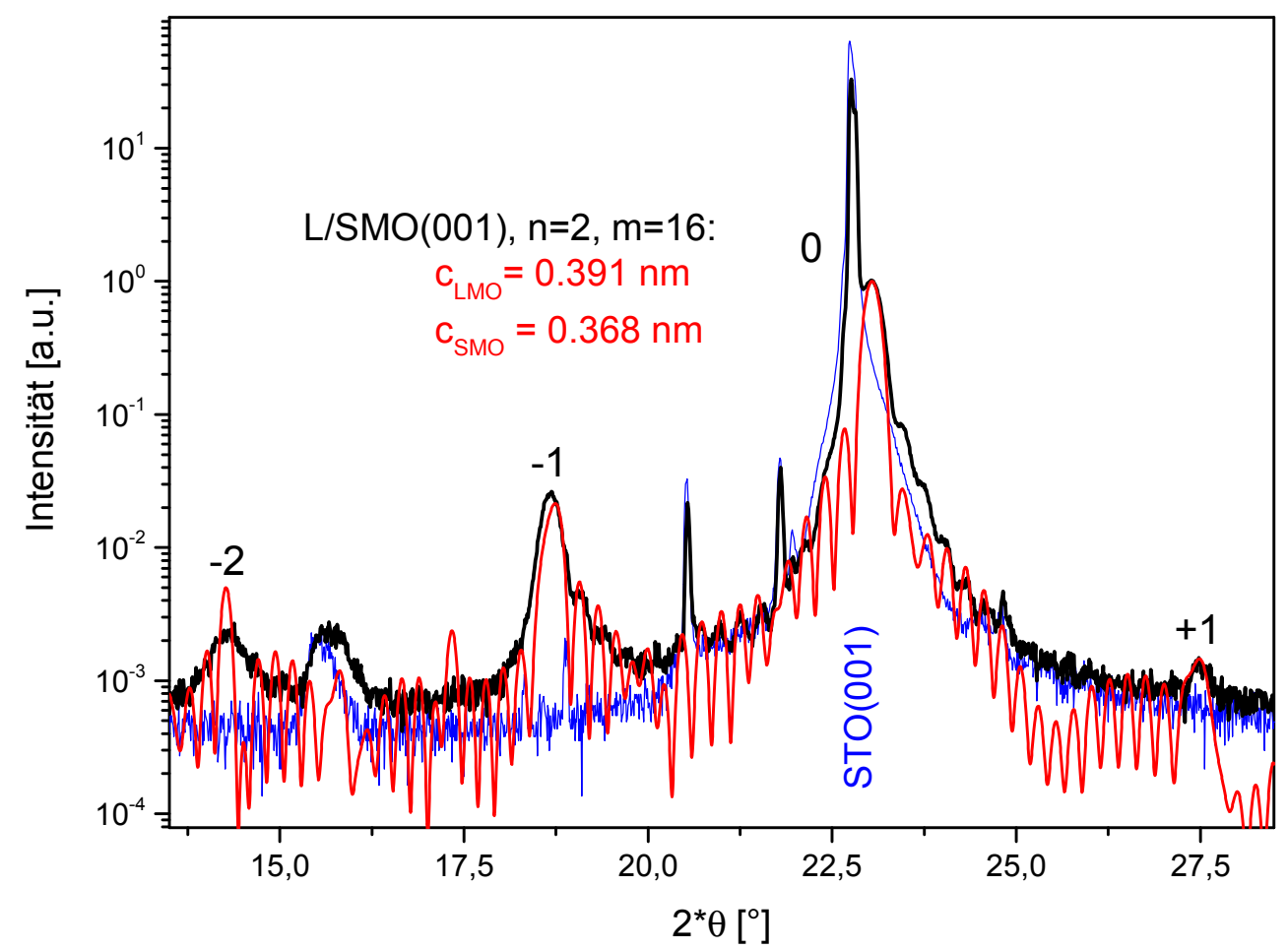

Abbildung 34: XRD Messung des L/SMO(001) Übergitters mit $n=2$ (schwarz) um den STO(001) Reflex. Es sind deutlich die Übergitterreflexe -2, -1 und +1 und die Laue-Oszillationen zu erkennen, die mit der XRD Simulation (rot) nachgebildet werden können. Neben den in Abb.33 angegebenen Simulationsparametern kann zusätzlich dieser Simulation die out-of-plane Gitterparameter von LMO und SMO gefunden werden. Zusätzlich ist die XRD Messung des Substrates in blau dargestellt.

in dem Modell gleichgesetzt. Dieses Modell funktioniert für LS/LMO(001), L/SMO(001) und für dickere LS/LMO(111) Übergitter. Es war nicht möglich ein Modell zu finden, das den Verlauf der XRR Messungen der L/SMO(111) Übergitter (exemplarisch in Abb.33 gezeigt) nachbilden kann. Die Bilagen-Dicken können für diese Filme dennoch aus der Lage der Überstrukturreflexe ermittelt werden. Die strukturellen Daten, die mittels XRR Simulation für alle Übergitter gewonnen werden konnten, sind in Tab.4.2.2 gezeigt. Die RMSOberflächenrauigkeit $\sigma_{o}$ bewegt sich zwischen $0.3 \mathrm{~nm}$ und $0.45 \mathrm{~nm}$, während die SubstratGrenzflächenrauigkeit $\sigma_{s}$ auf einem konstanten Wert von $0.6 \mathrm{~nm}$ stagniert. Der Fehler des zuletzt benannten Wertes muss jedoch, aufgrund des Einflusses dieses Parameters auf die XRR Simulation, mit $\pm 0.2 \mathrm{~nm}$ angegeben werden. Die Grenzflächenrauigkeit zwischen den Manganatlagen $\sigma_{i}$ ist für die L/SMO(001) Filme atomar scharf, wie es bereits in den TEM Messungen beobachtet wurde (siehe Abb.31). Demgegenüber sind die Werte für $\sigma_{i}$ für die LS/LMO(111) Übergitter, im Vergleich zu den TEM Aufnahmen (siehe Abb.30), eher zu klein. Die größten Rauigkeiten wurden in den LS/LMO(111) Filmen gefunden. 
4 A-Platz Ordnung in ferromagnetischen halbmetallischen Manganaten

Die gemittelten pseudokubischen Gitterparameter für alle Übergitter liegen zwischen $c \sim 0.385-0.386 \mathrm{~nm}$ und sind damit gleich dem Gitterparameter für LSMO. Für die atomar scharfen L/SMO(001) Übergitter können aus den XRD Messungen die out-of-plane Gitterparameter der LMO und SMO Komponente separat ermittelt werden. Wie in Abb.34 für das L/SMO(001) $n=2$ Übergitter dargestellt, sind die Überstrukturreflexe links und rechts von dem Gitterparameter nullter Ordnung stark unsymmetrisch. Mittels einer XRD Simulation nach Kap. 3.2.2 unter der Verwendung der aus den XRR Simulationen bekannten Parametern, können die angesprochenen Gitterparameter bestimmt werden. Dabei ist die Asymmetrie der Übergitterreflexe -1 und +1 durch die Simulation nur dann anzupassen, wenn das SMO stark verspannt ist mit $c_{S M O}=0.368 \mathrm{~nm}$. Entsprechend der Simulation ist das LMO entspannter, als im Falle eines dicken LMO Filmes, mit $c_{L M O}=0.391 \mathrm{~nm}$.

\subsubsection{Magnetische und elektrische Eigenschaften der LSMO Übergitter}

Alle Übergitter wurden, durch temperaturabhängige Messungen des spezifischen elektrischen Widerstands $\rho(T)$, der Magnetisierung $M(T)$ und durch $M(H)$-Hysterese bei tiefen Temperaturen, elektrisch und magnetisch charakterisiert. Im folgenden werden die Charakteristika der elektrisch- und magnetisch-hochwertigsten Bulk-LSMO Filme (höchste Übergangstemperatur, geringster spez. Widerstand, siehe Abb.27) mit den Übergittern verglichen und als Übergitter mit $n=0$ definiert. Entsprechend werden die (111)-Übergitter mit dem pulsiert-deponierten LSMO Film verglichen.

Die Messungen des elektrischen Widerstands wurden parallel zu den Übergitter-Lagen durchgeführt. Alle Übergitter zeigen einen Metall/Isolator- (oder Metall/Metall-) Übergang bei einer Temperatur $T_{M I}{ }^{16}$ und kein isolierendes Verhalten bei tiefen Temperaturen. Exemplarisch dafür ist in Abb.35 der spez. Widerstand als Funktion der Temperatur $\rho(T)$ für die gesamte L/SMO-Serie auf STO(111) präsentiert. Wie auch in den Anderen hier präsentierten Übergitter-Serien vergrößert sich der Widerstand mit steigenden $n$ für das gesamte betrachtete Temperaturintervall. In der Regel verschiebt sich die Übergangstemperatur mit steigenden $n$ zu niedrigeren Temperaturen. Diese $\rho(n)$ - bzw. $T_{M I}(n)$-Abhängigkeit ist in Abb.35 durch den gepunkteten Pfeil skizziert. Jedoch gibt es Ausnahmen von dieser Regel: Die Übergangstemperatur des L/SMO(111) $n=2$ Übergitters (blauer Pfeil in Abb.35) ist bemerkenswerterweise höher, als die Übergangstemperatur des pulsiert gewachsenen LSMO(111) Filmes.

Die Abhängigkeit der Curie-Temperatur $T_{C}$ von $n$ ist proportional zum $T_{M I}(n)$ Verhalten.

\footnotetext{
${ }^{16} T_{M I}$ wurde über das Maximum des $\alpha_{R}$-Wertes bestimmt.
} 


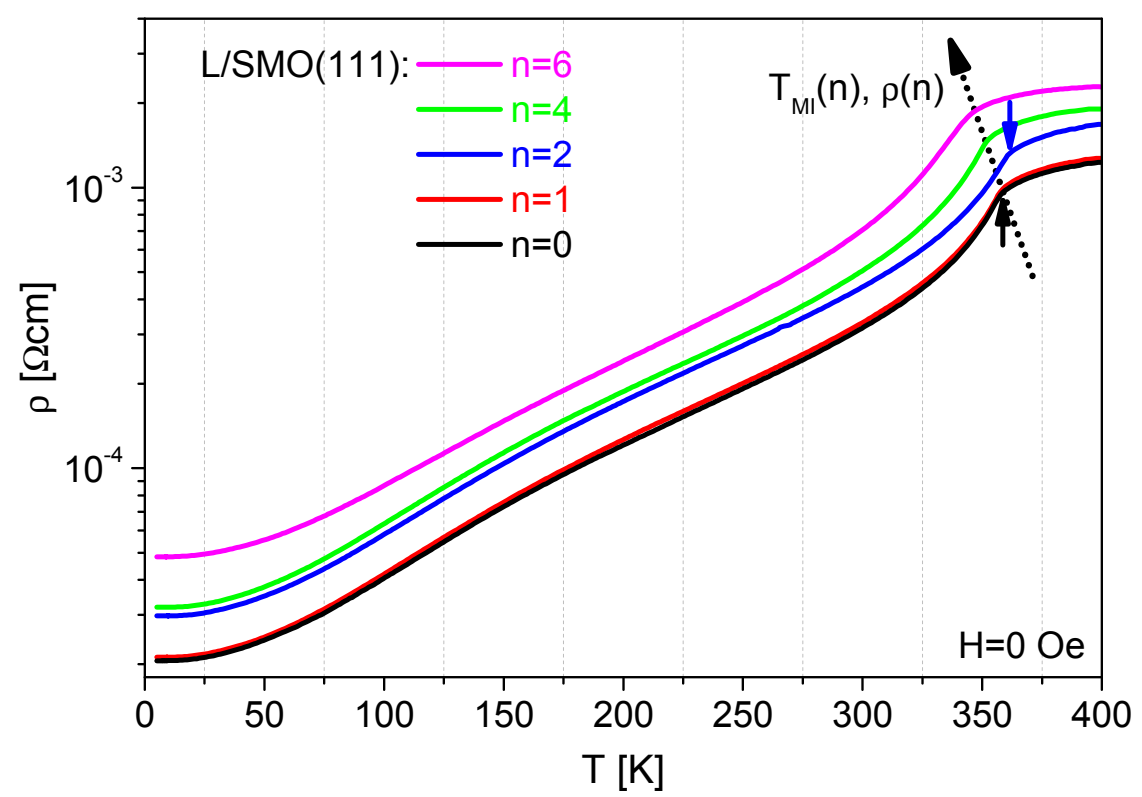

Abbildung 35: Die Temperaturabhängigkeit des spezifischen elektrischen Widerstandes $\rho$ der L/SMO(111) Übergitter mit $n=0 \ldots 6$ bei Nullfeld. Die L/SMO $n=0$ Probe ist der gemischte LSMO Film, der pulsiert auf STO(111) gewachsen wurde. Der gepunktete Pfeil deutet die tendenzielle Entwicklung der Metall/Isolator-Übergangstemperatur $T_{M I}$ und $\rho$ mit ansteigendem $n$ an. Der $T_{M I}(n=2)$ Wert (blauer Pfeil) weicht von dieser Tendenz ab und ist größer als der $T_{M I}(n=0)$ Wert (schwarzer Pfeil).

Angesichts dieser bekannten Tatsache wurden in Abb.36 die Magnetisierungs-Abkühl-Kurven der verschiedenen Typen von Übergittern für die näherungsweise gleiche Bilagen-Dicke bei einem externen Feld von 100Oe aufgetragen. In Abb.36 a) ist für die Proben auf STO(001) deutlich zu erkennen, dass das $T_{C}$ von L/SMO mit $n=1$ kleiner ist, als das $T_{C}$ von LS/LMO mit $n=1$, welches wiederum kleiner ist, als die Curie Temperatur des LSMO Bulk-Films. Auf STO(111) ist diese Abhängigkeit, wie es sich schon in den elektrischen Daten angekündigt hat (siehe Abb.35), nicht zu beobachten. In Abb.36 b) ist zu sehen, dass das $T_{C}$ von LS/LMO mit $n=2$ leicht kleiner ist als das $T_{C}$ des pulsiert gewachsenen LSMO Films, während die Curie-Temperatur von L/SMO mit $n=2$ größer ist, als die des Bulk-Films. Die eingebetteten Diagramme in Abb.36 zeigen die $M(H)$ Hysterese-Schleifen der Übergitter und der entsprechenden LSMO Bulk-Filme bei $T=5 K$. Bezogen auf die Substratorientierung zeigen die Filme nahezu die gleichen Eigenschaften: Die Sättigungsmagnetisierung bei hohen Feldern und tiefen Temperaturen zeigt auf beiden Substraten einen Wert von $\sim 3.7 \mu_{B} / M n$. Das Koerzitivfeld liegt für alle in Abb.36 a) präsentierten Filme auf $S T O(001)$ bei weniger als $20 O e$, bzw. für alle in Abb.36 b) präsentierten Filme auf $S T O(111)$ bei weniger als $2 O e$.

In Abb.37 sind die wichtigsten, magnetischen Eigenschaften aller hier vorgestellten LSMO Filme noch einmal zusammengefasst. Für die Übergitter auf STO(001) fällt die Curie- 
4 A-Platz Ordnung in ferromagnetischen halbmetallischen Manganaten

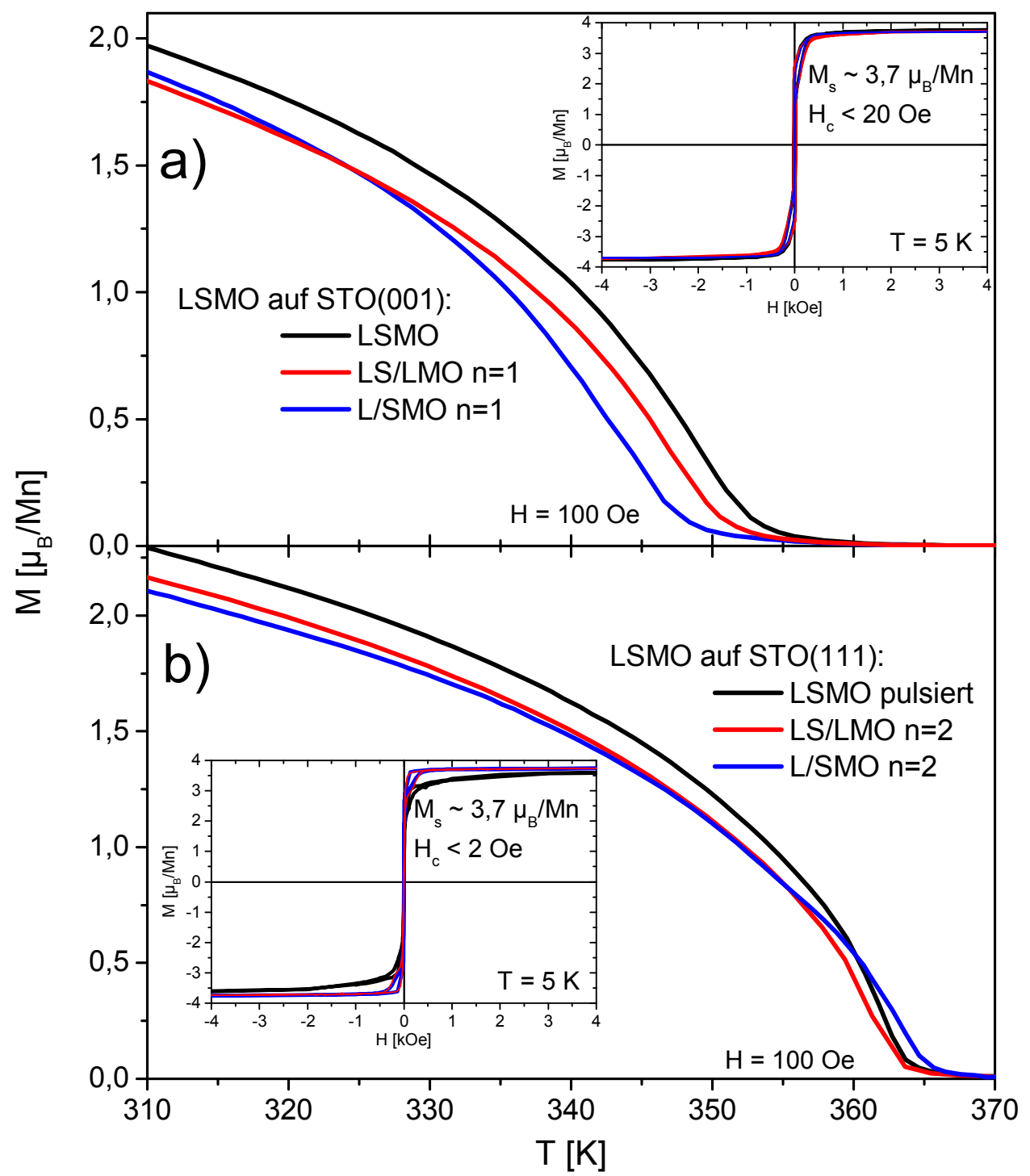

Abbildung 36: Die Abkühl-Magnetisierungskurven für a) $L S M O(n=0)$ (schwarz), $L S / L M O(n=$ 1) (rot) und $L / S M O(n=1)$ (blau) auf $S T O(001)$, bzw. b) $L S M O(n=0$, pulsiert deponiert) (schwarz), $L S / L M O(n=2)$ (rot) und $L / S M O(n=2)$ (blau) auf $S T O$ (111) bei einem externen Feld von 100Oe. Die eingebetteten Diagramme zeigen die $M(H)$ Kurven nach dem feld-einkühlen bei $5 K$. 
Temperatur mit steigender Bilagen-Dicke, die proportional zum Parameter $n$ ist, ab. Dabei fällt das $T_{C}$ für die L/SMO Übergitter stärker ab, als für die LS/LMO Übergitter. Insbesondere existiert kein Übergitter auf STO(001), dass die gleiche oder eine höher Curie-Temperatur aufweist, als der LSMO Bulk-Film. Der Verlauf der Curie-Temperatur als Funktion der Bilagen-Dicke der Übergitter auf STO(111) zeigt keinen Unterschied zwischen den L/SMO und LS/LMO Übergittertypen. Interessanterweise steigt das $T_{C}$ als Funktion der BilagenDicke bzw. des Parameters $n$ leicht an, oder befindet sich auf einem Niveau bis zu einem Wert von $n=2$. Für $n>2$ fällt $T_{C}$ wiederum monoton ab, jedoch nicht so stark wie für die (001) Übergitter. Die Sättigungsmagnetisierung $M_{s}=M(5 K, 10 k O e)$ liegt für alle bis auf ein Übergitter innerhalb des typischen Fehlers für MPMS Messungen (siehe Kap. 3.2.6) im Bereich der maximalen theoretischen Sättigungsmagnetisierung für LSMO von $3.67 \mu_{B} / M n$ (blaue gepunktete Linie in Abb.37). Das abweichende Übergitter ist das L/SMO mit $n=3$ auf STO(001), mit einer Sättigungsmagnetisierung von 3.05 $\mu_{B} / M n$ (in Abb.37 markiert durch den schwarzen Pfeil). Diese Probe zeigt auch die niedrigste Curie-Temperatur und das höchste Koerzitivfeld von allen in dieser Arbeit untersuchten Übergittern.

\subsubsection{Diskussion der LSMO Übergitter}

Die strukturellen Daten zeigen, dass alle Übergitter in einer hohen kristallinen Qualität, epitaktisch auf dem Substrat aufgewachsen sind. Allgemein zeigt die Bilagen-Dicke in manchen Proben eine Abweichung von maximal 10\% von den angestrebten Werten. Dieser Fehler liegt in dem Bereich, der für diese Größenordnung der einzelnen Lagen auch in LSMO Übergittern, die mit der Molekularen Gasphasenabscheidung (MBE) gewachsen wurden, beobachtet werden kann [116]. Die Grenzflächenrauigkeiten $\sigma_{i}$ zwischen den einzelnen Manganaten streuen für alle Übergitter über einen relativ breiten Bereich: Während die L/SMO(001) atomar scharfe Grenzflächen mit $\sigma_{i}=0.0 \ldots 0.2 \mathrm{~nm}$ zeigen, kann für LS/LMO(001) Übergitter auch $\sigma_{i}=0.6 \mathrm{~nm}$ beobachtet werden (Tabelle 1 ).

Die atomar scharfen Grenzflächen der L/SMO(001) Übergitter werden auch in L/SMO(001) Übergittern beobachtet, die mit Hilfe der MBE Methode präpariert wurden [105, 106, 116]. Sowohl in den TEM Messungen als auch in den XRR Simulationen wurde gezeigt, dass in den L/SMO(001) Filmen die LMO Lagen dicker und die SMO Lagen dünner sind, als die angestrebten Werte für diese Schichtdicken. Da in den TEM EELS Messungen (Abb.31) nur das La und nicht das Sr observiert werden kann, können kristalline Fehler, wie Substratstufen oder leichte Rauigkeit an der Grenzfläche durch nicht vollständige Monolagen, dazu führen, dass LMO dicker und SMO dünner erscheint. Entsprechendes gilt auch für 
4 A-Platz Ordnung in ferromagnetischen halbmetallischen Manganaten

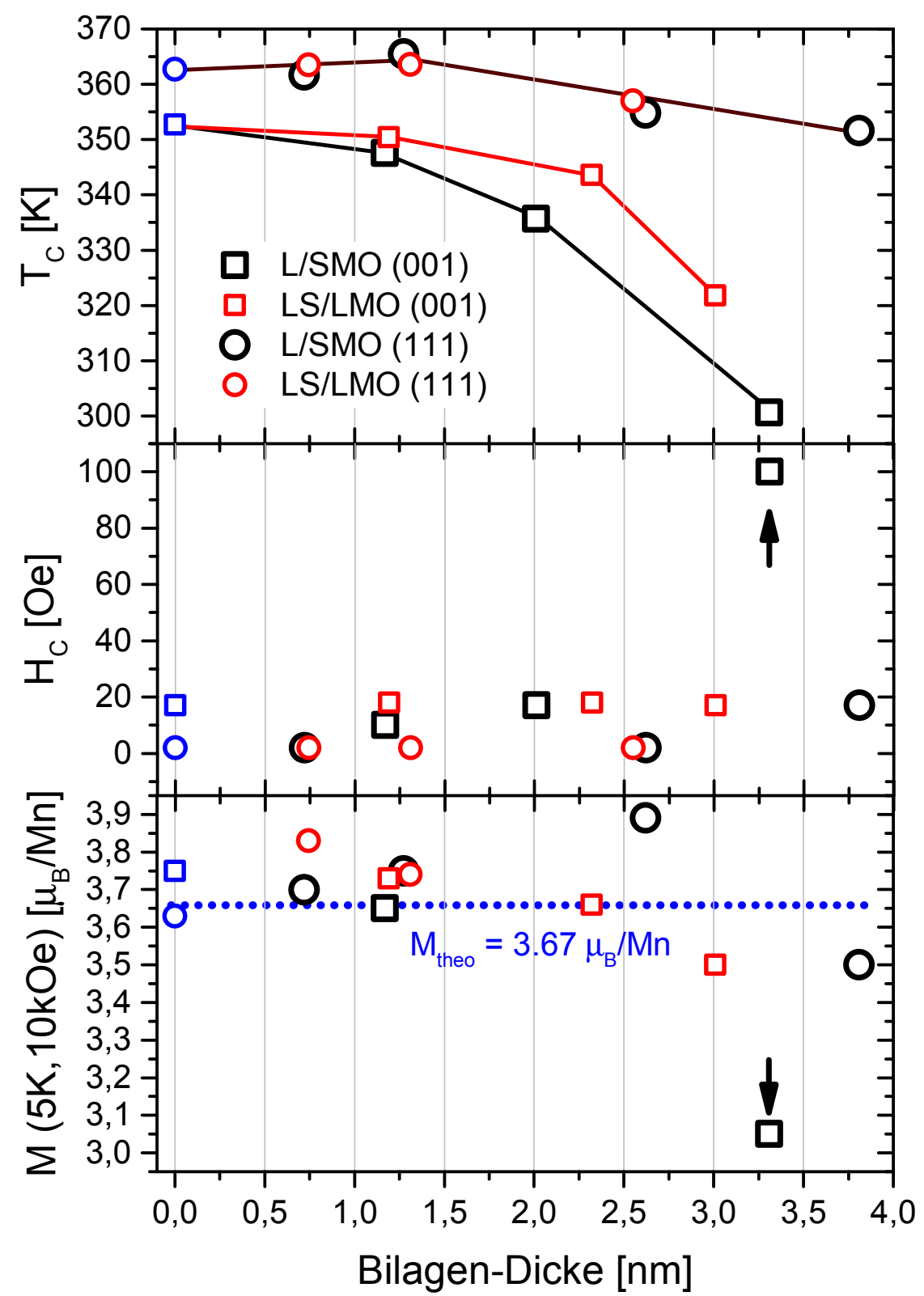

Abbildung 37: Die Curie Temperatur $T_{C}$, das Koerzitivfeld $H_{c}$ und die Sättigungsmagnetisierung bei tiefen Temperaturen und hohen Feldern $M(5 K, 10 k O e)$ als Funktion der Bilagen-Dicke der L/SMO und LS/LMO Übergitter auf STO(001) und STO(111). Die Daten der einphasigen LSMO Filme sind in blau dargestellt. Die theoretische maximale Sättigungsmagnetisierung von LSMO mit der Sr-Dotierung $x=0.33$ ist durch die gepunktete blaue Linie dargestellt. Das L/SMO Übergitter mit $n=3$ auf $S T O(001)$ zeigt keine volle Sättigung und ein besonders hohes $H_{c}$ (markiert durch die schwarzen Pfeile). 
die TEM Messungen der Proben auf STO(111) (Abb.30). Für die L/SMO(001) Proben ist es genauso möglich, die XRR Messungen durch Simulationen mit einem idealen LMO/SMO Verhältnis von $2: 1$ mit asymmetrischen $\sigma_{i}$ anzupassen. Die TEM Messungen (Abb.31) als auch die Ellipsometrie-Daten, die während des Depositionsprozesses aufgenommen wurden, können auch so interpretiert werden, dass die Grenzflächen in L/SMO asymmetrisch sind (letzteres wird genauer in der Dissertation von Markus Jungbauer behandelt [36]). In der Literatur kann man sowohl asymmetrisch-raue Grenzflächen als auch ein nicht angestrebtes LMO/SMO Missverhältnis in L/SMO finden [116]. Die genaue Struktur der L/SMO Übergitter kann somit nicht vollständig aufgelöst werden. Aufgrund der scharfen Grenzflächen war es in diesen Übergittern möglich die out-of-plane Gitterparameter von LMO und SMO zu bestimmen: $c_{L M O}=0.391 \mathrm{~nm}$ und $c_{S M O}=0.368 \mathrm{~nm}$. Ähnliche Werte wurden bereits in L/SMO(001) Übergittern gefunden [110].

Die XRR Messungen der L/SMO(111) Übergitter können nicht durch die Simulation angepasst werden: In Abb.25 ist erkennbar, das SMO auf der (111) Oberfläche stark aufraut. Dies hat zur Folge, dass die (111)-Grenzflächen in den L/SMO(111) Übergittern auch aufrauen, was auch in der in situ Ellipsometrie beobachtet wird [36]. Bilden sich, durch den in Kap. 4.2.1 aufgezeigten Ladungsausgleich, in SMO \{001\} Facetten aus, so kann es in diesen Übergittern auch zu einer Ordnung kommen die, aufgrund der Geometrie, nicht von den XRR oder XRD Messungen aufgelöst werden kann. Entsprechend ist es auch nicht möglich ein Modell für die XRR Simulationen zu finden, um die XRR Messungen anzupassen.

In den L/SMO Übergittern wurde beobachtet, dass LMO sehr scharf auf SMO gewachsen werden kann. Weiterhin wurde beobachtet, dass sowohl LMO als auch LSMO:0.5 nicht auf STO(001) oder STO(111) aufraut. Somit kann nicht geklärt werden, warum die Grenzflächen zwischen LMO und LSMO:0.5 sowohl auf STO(001) als auch auf STO(111) eine recht große Rauigkeit zeigen, die mit wachsendem $n$ weiter ansteigt. Die in den TEM Messungen des LS/LMO(111) Übergitters mit $n=4$ beobachtete Bilagen-Dicke, als auch das Verhältnis von LMO/LSMO:0.5 konnte in den XRR Messungen nicht bestätigt werden. Während die Bilagen-Dicke über die XRR Simulation genau bestimmt werden kann, ist das für das LMO/LSMO:0.5 Verhältnis, aufgrund der relativ rauen Grenzflächen, nicht möglich. Da die XRR Methode eine globale Messung der Struktur darstellt, ist entsprechend in der lokalen TEM Messung ein nicht repräsentativer Ausschnitt der Probe dargestellt.

In den strukturellen Messungen konnte die gewünschte Übergitterstruktur bestätigt und die Rauigkeit an der Grenzfäche charakterisiert werden. Jedoch können nicht alle Fragen über die genaue Struktur (Dicke der LMO, SMO oder LSMO:0.5 Lagen, asymmetrische Grenzflächen) durch die TEM und XRR Messungen beantwortet werden. 
Bevor mit der Diskussion der elektrischen und magnetischen Eigenschaften der Übergitter fortgefahren wird, muss verstanden sein, dass in diesen Übergittern, neben der Beeinflussung der physikalischen Eigenschaften durch eine Ordnung der Gitterverzerrung mit der A-Platz Kationen Ordnung, auch eine Änderung der $e_{g}$ Ladungsdichte der $M n$ Ionen einher geht (ein grundlegender Unterschied zum LCMO/PCMO Übergittersystem in Kap. 4.1). Damit kann auch der Anstieg des spezifischen Widerstandes und das Absinken der Übergangstemperatur verstanden werden, beides wurde mit steigenden $n$ in allen Übergittern beobachtet: Für große $n$ wird das $e_{g}$-elektrische System aufgetrennt, es entsteht eine modulierte Besetzung der $e_{g}$ Orbitale. Im LSMO Phasendiagramm für dünne Filme (siehe Abb.28) befindet sich das System nicht mehr im optimalen Punkt mit höchster elektrischer Leitfähigkeit und Curie-Temperatur, sondern periodisch links und rechts neben diesem Punkt. Entsprechend steigt der Widerstand und die Übergangstemperatur nimmt ab. Vergleicht man die Breite dieses Maximums für die verschiedene Substratorientierungen ist auch ersichtlich, warum das $T_{C}$ für Übergitter auf STO(001) stärker mit steigenden $n$ abfällt. Diese Modulation ist durch die scharfen Grenzflächen und die stärkere Trennung der $e_{g}$ Elektronenbesetzung $^{17}$ in L/SMO(001) größer als in den raueren $L S / L M O$ Übergittern. Demzufolge ist auch zu verstehen, warum das $T_{C}(n)$ für L/SMO(001) stärker sinkt, als das $T_{C}(n)$ für LS/LMO(001). Die raueren Grenzflächen der (111)-Übergitter und das breitere $T_{C}$-Maximum im Phasendiagramm für (111)-LSMO Filme führen dazu, dass diese Trennung der $T_{C}(n)$ Kurven für L/SMO(111) und LS/LMO(111) Übergitter für die observierten Bilagen-Dicken nicht beobachtet werden kann. Aufgrund des großen Einflusses der $e_{g}$ Elektronenbesetzung auf die physikalischen Eigenschaften, bewirkt die A-Platz Ordnung in (100)-Übergittern als auch in (111)-Übergittern mit $n \geq 4$ keine Optimierung der Übergangstemperatur. Dieser Besetzungsunterschied ist analog zu einer Ladungsordnung, die dem DE entgegenwirkt. In diesem Zusammenhang ist es auch zu verstehen, warum es gelungen ist die Néel Temperatur durch APlatz Ordnung in $\mathrm{La}_{1 / 3} \mathrm{Sr}_{2 / 3} \mathrm{MnO}_{3}$ zu erhöhen [9]. Diese magnetische Wechselwirkung läuft über den SE, der durch die Ladungsordnung begünstigt wird. Bemerkenswerterweise wird der Unterschied in der $e_{g}$-Elektronenbesetzung bereits in dem L/SMO(001) Übergitter mit $n=3$ bereits so groß, sodass das SMO AFM Eigenschaften aufweist. Diese zeigen sich durch den Abfall der Sättigungsmagnetisierung auf 3.0 $\mu_{B} / M n$ und durch den Anstieg des Koerzitivfeldes auf $\sim 1000$ e (verursacht durch das magnetische pinning des Löcherdotierten FM LMOs durch das AFM SMO). An dem Punkt kann man bei diesem Übergitter nicht mehr von einem Kationen-geordneten LSMO sprechen, hier haben die Grenzflächenphänomene einen großen Einfluss: Insbesondere kann eine zweite ferromagnetische Phase an der Grenzfläche zwischen LMO und SMO beobachtet werden. Diese Thematik wird in der Dissertation von Markus

\footnotetext{
${ }^{17}$ Nominell hat LMO eine $e_{g}$ Besetzung von 1, LSMO:0.5 von 0.5 und SMO von 0. Für Übergitter mit $n=1$ ist die Besetzung der Bilage entsprechend für L/SMO: 1/1/0 und für LS/LMO: 0.5/0.5/1.
} 
Jungbauer abgehandelt [36]. Bei allen übrigen Übergittern kann von A-Platz (teil)geordneten LSMO gesprochen werden, da hier die elektrische Verteilung der $e_{g}$-Besetzung groß genug ist, damit eine Sättigungsmagnetisierung von $\sim 3.67 \mu_{B} / M n$ erreicht werden kann.

In der Literatur können Daten für L/SMO(001) Übergitter gefunden werden [105, 106]: im Vergleich ist die Curie-Temperatur und die Sättigungsmagnetisierung in der Literatur niedriger und beide Parameter fallen stärker mit steigenden $n$ ab. Es wird auch ein isolierendes Verhalten bei tiefen Temperaturen für L/SMO Übergitter mit $n>2$ beobachtet, dass mit dem Einsetzen des AFM in SMO in Verbindung gebracht wird [107]. Diese isolierende Phase kann in den hier aufgeführten Übergittern nicht beobachtet werden. Möglicherweise unterscheiden sich die hier präsentierten Übergitter jedoch von der Literatur, durch die unterschiedliche Sauerstoffkonzentration, bedingt durch die verschiedenen Depositionsmethoden (MAD und MBE). Die These einer höheren Sauerstoffkonzentration in den MAD Proben wird auch durch die im Vergleich kleineren out-of-plane Gitterkonstanten in Bulk-Filmen [109] als auch in Übergittern [110] unterstützt. Eine niedrigere Sauerstoffkonzentration würde den DE negativ beeinflussen [117] und könnte die kleineren Werte für $T_{C}$ und $M_{s}$ sowie das Einsetzen eines isolierenden Verhaltens bei tiefen Temperaturen der Übergitter in der Literatur erklären.

Die (111)-Übergitter mit $n<4$ unterscheiden sich strukturell deutlich von dem LSMO Bulk-Film, durch die Anwesenheit der Überstrukturreflexe in XRR und XRD. Zudem kann die A-Platz Ionen-Modulation in den TEM Messungen beobachtet werden. Elektrisch und magnetisch unterscheiden sich diese Übergitter jedoch nur geringfügig von dem LSMO BulkFilm. Erstaunlicherweise ist für das L/SMO Übergitter mit $n=2$ eine um $3 K$ höhere Übergangstemperatur zu beobachten. Die ungewöhnliche Struktur dieser L/SMO(111) Übergitter, durch das Aufrauen des SMOs wurde bereits angesprochen. Möglicherweise konnte in dieser Struktur eine A-Platz Ordnung eingestellt werden, die zum einen die Übergangstemperatur durch A-Platz Ordnung der Gitterverspannung [7,8] erhöht und zum anderen durch $e_{g}$-Ladungstrennung erniedrigt.

In den untersuchten Ansätzen zur A-Platz Ordnung in LSMO, hat die $e_{g}$-Ladungstrennung meist einen größeren Einfluss auf die physikalischen Eigenschaften, als die strukturelle Ordnung. Grundsätzlich scheint die Erhöhung der Übergangstemperatur durch künstliche Ordnung der A-Platz Ionen in L/SMO(111) möglich zu sein. Um einen größeren Effekt durch die Ordnung zu erzielen, muss die $e_{g}$ Elektronenbesetzung von der Abhängigkeit zu den A-Platz Ionen gelöst werden: Dies kann durch einen Multilagen-Ansatz mit isovalenten Manganaten realisiert werden. 



\section{B-Platz Ordnung in ferri-/ferromagnetischen Dop- pelperowskiten}

In der Klasse der Doppelperowskite (DP) mit der generellen Formel $A_{2} B B^{\prime} O_{6}$ sind sowohl ferromagnetische Isolatoren [10, 118], multiferroische Materialien [119] als auch ferrimagnetische Halbmetalle mit Curie-Temperaturen weit über Raumtemperatur [11, 120] vertreten. Aus diesem anwendungsspezifischen Gesichtspunkt, wie auch aus der Tatsache, dass die genannten Eigenschaften stark von der Ordnung der Übergangsmetallionen $B-B^{\prime}$ in diesen Materialen abhängen, erregen die DP seit einigen Jahren ein reges wissenschaftliches Interesse. Viele dieser DP haben die Eigenschaft, dass ein B-Platz Ion in einer Valenz vorkommt, die bei hohen Sauerstoffpartialdrücken unstabil ist. Aus diesem Grund konnten bisher keine DPs mit der MAD Methode (erfolgreich) präpariert worden. In dieser Arbeit wurde, aufgrund dieser Problematik, zunächst mit dem ferromagnetische Isolator $\mathrm{La}_{2} \mathrm{CoMnO}_{6}$ ein DP System gewählt, dessen Einzelkomponenten sich bereits mit der MAD präparieren ließen. Das Hauptaugenmerk lag dabei auf der Untersuchung und Optimierung der B-Platz Ordnung und deren Auswirkung auf die ferromagnetischen Eigenschaften des Materials. Um auch ferrimagnetische, halbmetallische DP wie $\mathrm{Sr}_{2} \mathrm{FeMoO}_{6}$ im MAD Verfahren herstellen zu können, wurde in dieser Arbeit die sauerstoffarme MAD (Low Oxygen MAD - LOMAD) entwickelt. Mit ihr ist die Kontrolle über die Valenz der Ionen mit Hilfe der Steuerung des Sauerstoffpartialdruckes gelungen. 



\subsection{B-Platz Ordnung in $\mathrm{La}_{2} \mathrm{CoMnO}_{6}$ Filmen}

Da sowohl dünne $\mathrm{LaMnO}_{3}$ (LMO) wie auch $\mathrm{LaCoO}_{3}$ (LCO) Schichten mit dem MAD Verfahren in hoher Qualität hergestellt werden können, wurde zunächst das $\mathrm{La}_{2} \mathrm{CoMnO}_{6}$ (LMCO) System gewählt, um die Frage zu klären, ob es möglich ist im MAD Verfahren B-Platz-geordnete DP Schichten zu synthetisieren. Wie in den meisten DPs, ordnen sich die B-Platz-Ionen aufgrund der Valenz- und Größenunterschiede in einer Kochsalzstruktur [121]. Das LMCO ist ein ferromagnetischer Isolator, dessen magnetisches Moment und CurieTemperatur stark von der Ordnung der B-Platz-Ionen abhängen: Während in der ungeordneten Phase eine Curie-Temperatur von $T_{C} \sim 130 \mathrm{~K}$ und eine Sättigungsmagnetisierung bei hohen Feldern und tiefen Temperaturen von $M_{s a t}<4 \mu_{B} / f . u \cdot{ }^{18}$ anzutreffen ist, findet man in der geordneten Phase $T_{C} \sim 220-230 K$ und $M_{\text {sat }} \sim 6 \mu_{B} / f . u$. [10].

\subsubsection{Elektronische Struktur von $\mathrm{La}_{2} \mathrm{CoMnO}_{6}$}

In der Literatur wird argumentiert, dass der Ferromagnetismus in LMCO aus einer Superaustauschwechselwirkung zwischen $\mathrm{Co}^{2+}$ und $\mathrm{Mn}^{4+}$ resultiert [10]. Dabei liegt das $\mathrm{Co}^{2+}$ in einer high-spin $3 d^{7}$ Konfiguration vor, d.h. beide $e_{g}$ Orbitale sind halb gefüllt und das Ion hat einen resultierenden Gesamtspin von $S=3 / 2$. Das $M n^{4+}$ liegt dagegen in der $3 d^{3}$ Konfiguration vor, damit sind alle $t_{2 g}$ Orbitale halb gefüllt und es liegt auch ein Gesamtspin von $S=3 / 2$ vor. Ordnet man diese Ionen in der Kochsalzstruktur, so ist jedes Mn-Ion über die Sauerstoffbrücke in jeder Richtung mit einem Co-Ion verbunden (und umgekehrt). Aus dieser Konfiguration resultiert nach der zweiten GKA Regel ein ferromagnetischer Superaustausch (siehe Kap. 2.2) mit einer Sättigungsmagnetisierung von $S=3 / 2+3 / 2 \bumpeq 6 \mu_{B} / f . u$. An diesem Punkt ist auch zu erwähnen, dass sowohl die Kopplung zwischen $\mathrm{Co}^{2+}-\mathrm{O}-\mathrm{Co}^{2+}$ als auch $\mathrm{Mn}^{4+}-\mathrm{O}-\mathrm{Mn}^{4+}$ nach der ersten GKA-Regel antiferromagnetisch sein soll. Somit koppeln Antiphasen-Punktdefekte wie auch Antiphasen-Grenzen antiferromagnetisch. Damit würde auch die Magnetisierung von zwei perfekt geordneten, ferromagnetischen Domänen, die durch eine antiferromagnetische Antiphasen-Grenze getrennt sind, im Nullfeld gegeneinander stehen [122]. Weiterhin wird in der Literatur diskutiert, dass in der ungeordnete Phase ein $e_{g}$-Elektron von dem $\mathrm{Co}^{2+}$ an das $\mathrm{Mn}^{4+}$ übertragen wird, wodurch intermediate-spin $\mathrm{Co}^{3+}$ mit der Konfiguration $t_{2 g}^{5} e_{g}^{1}$ und high-spin $\mathrm{Mn}^{3+}$ mit der Konfiguration $t_{2 g}^{3} e_{g}^{1}$ entsteht [10]. Dabei wird die Elektronenkonfiguration des $\mathrm{Co}^{3+}$ durch lokale Jahn-Teller Verzerrungen stabilisiert $[123,124]$. Thermoelektrische Messungen lassen darauf schließen, dass die $e_{g}$ Orbitale von $\mathrm{Co}^{3+}$ wie auch von $\mathrm{Mn}^{3+}$ vibronisch besetzt sind [10], d.h. das sich das Elektron mit

\footnotetext{
${ }^{18}$ f.u. $=$ Formeleinheit, doppelte pseudokubische Perowskit-Einheitszelle
} 
der gleichen Wahrscheinlichkeit in beiden $e_{g}$ Orbitalen aufhält. Weiterhin wird angenommen, dass die orbitalen Elektronenkonfigurationen über das Gitter durch den Jahn-Teller-Effekt, miteinander korreliert sind. Dies hat zur Folge, dass die zweite GKA Regel erfüllt ist. Somit koppeln $\mathrm{Co}^{3+}-\mathrm{O}-\mathrm{Co}^{3+}, \mathrm{Co}^{3+}-\mathrm{O}-\mathrm{Mn}^{3+}$ als auch $\mathrm{Mn}^{3+}-\mathrm{O}-\mathrm{Mn}^{3+}$ über den vibronischen Superaustausch [69, 125, 126] schwach ferromagnetisch. Sowohl die Valenzen, als auch die Elektronenkonfigurationen sind jedoch bisher nicht vollständig verstanden: Neutronenstreuung, Röntgenabsorptionsspektroskopie (XAS), zirkularer magnetischer Röntgendichroismus (XMCD) und Röntgenphotoelektronenspektroskopie bestätigen eine $\mathrm{Mn}^{4+} / \mathrm{Co}^{2+}$ Ordnung in bulk- und nanokristallinen Proben mit einer Curie-Temperatur von $T_{C} 220$ - $230 \mathrm{~K}$, während eine $\mathrm{Mn}^{3+} / \mathrm{Co}^{3+}$ Konfiguration in ungeordneten Proben mit niedrigeren CuriePunkt auftritt [127-132]. Jedoch präsentierte zuletzt die Gruppe um G.A. Sawatzky XASDaten von dünnen LMCO Filmen, die darauf schließen lassen, dass unabhängig von dem Ordnungsgrad der Probe, immer eine $\mathrm{Co}^{2+} / \mathrm{Mn}^{4+}$ Konfiguration vorliegt [133].

\subsubsection{Präparation von $\mathrm{La}_{2} \mathrm{CoMnO}_{6}$ mit MAD}

Laut der Literatur bereitete es in früheren Arbeiten große Schwierigkeiten ein geordnetes LMCO System zu synthetisieren, das an der Zeitspanne zwischen den ersten Versuchen und der tatsächlichen Realisierung fest gemacht werden kann: Während die Kation-ungeordnete Phase von LMCO bereits 1966 als Bulk-Polykristall von G.H. Jonker synthetisiert und untersucht wurde [134], gelang die Synthese einer fast reinen Kation-geordneten Phase erst 2002 in der Gruppe von J.B. Goodenough [10]. Es hat sich herausgestellt, dass die Ordnung in Bulk-Kristallen wie auch in dünnen Filmen stark von der Sauerstoffkonzentration und der Synthesetemperatur abhängt [10, 119, 135-138]. Die optimale Synthesetemperatur liegt dabei zwischen 600 und $1100^{\circ} \mathrm{C}$. Die geordneten Bulk-Kristalle wurden in einer Sauerstoffatmosphäre von bis zu 3bar über mehrere Tage ausgelagert, während geordnete dünne Schichten im PLD-Verfahren unter sehr hohen Sauerstoffdrücken von 0.25 - 1.1mbar hergestellt und unter 1bar Sauerstoffatmosphäre langsam von der Depositionstemperatur abgekühlt wurden. Aus dieser Vorbetrachtung heraus ist das MAD Verfahren, aufgrund des sehr hohen Sauerstoffpartialdruckes von 200mbar (unter Pressluft) während der Deposition, eine ideale Alternative zur Herstellung von dünnen, geordneten LMCO Schichten, neben der PLD. Aufgrund einer Gitterfehlanpassung von $~ 1 \%$ eignet sich $\mathrm{SrTiO}_{3}$ (STO) als Substrat.

Um die stöchiometrischen Parameter (siehe Kap. 3.1) für LMCO zu bestimmen, wurde zunächst das molare $L a / M n$-Verhältnis, wie in dem vorigen Kapitel beschrieben, bestimmt. Dieses Verhältnis wurde auch für die Deposition von LCO verwendet. In Abb.38 a) ist 

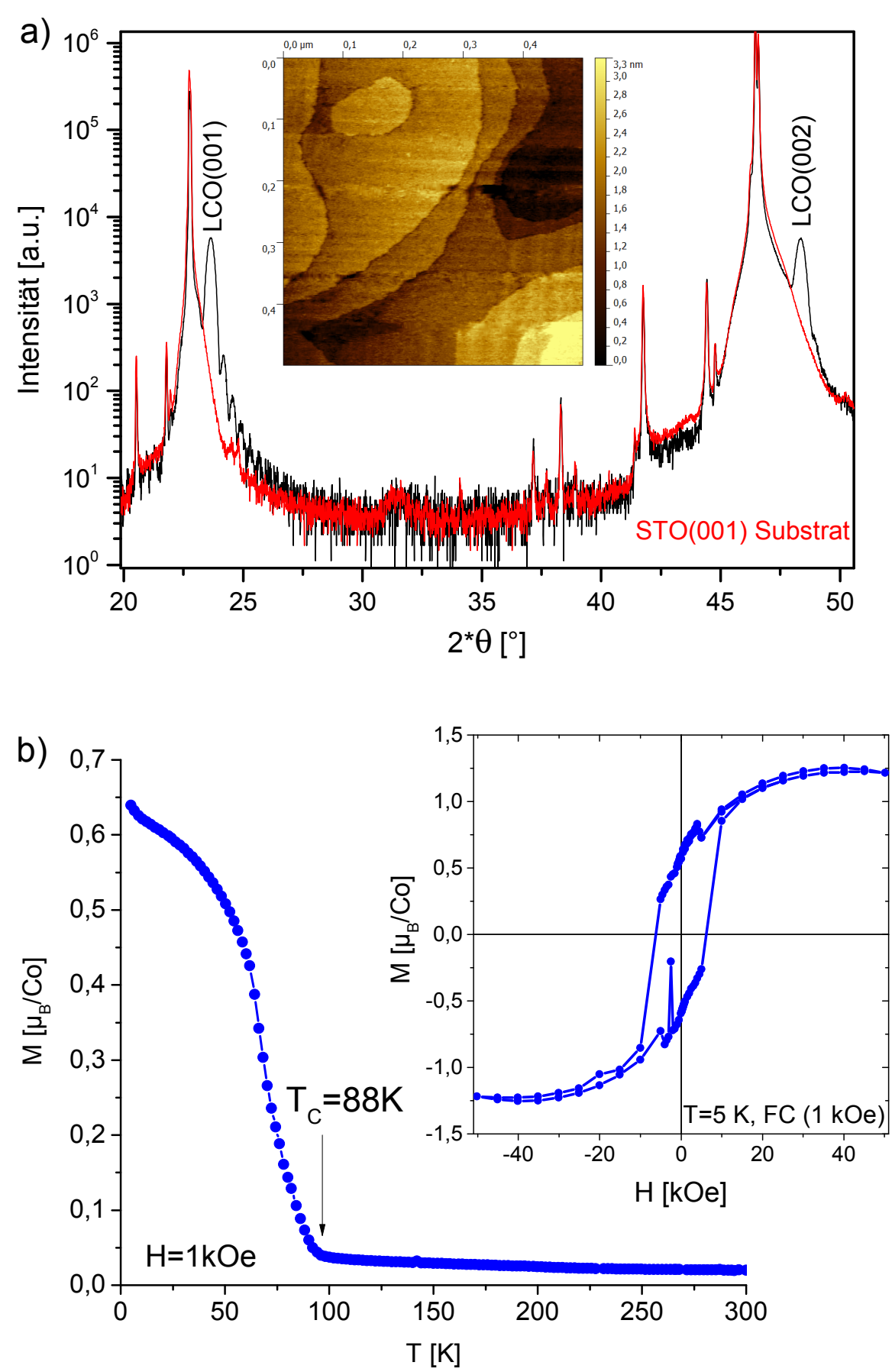

Abbildung 38: a) XRD Messung für einen 30nm LCO Film auf STO(001) Substrat. Eine Leersubstrat-Messung ist in rot gezeigt. Neben der (00l) sind keine weiteren Orientierungen oder Fremdphasen vorhanden. Das eingebettete Diagram zeigt eine $500 \times 500 \mathrm{~nm}^{2}$ STM Aufnahme dieser Probe. b) Magnetisierung $M$ in Abhängigkeit von der Temperatur T für einen $30 \mathrm{~nm} \mathrm{LaCoO}_{3} \mathrm{Film}$ bei einem angelegten externen Feld von $H=1 k O e$. Das eingebettete Diagram, zeigt die $M(H)$ Hysterese Kurve nach dem Feldeinkühlen bei $1 k O e$ auf $5 K$. Die Sättingungsmagnetisierung beträgt $M_{s}=1.25 \mu_{B} / C o$. 
5 B-Platz Ordnung in ferri-/ferromagnetischen Doppelperowskiten
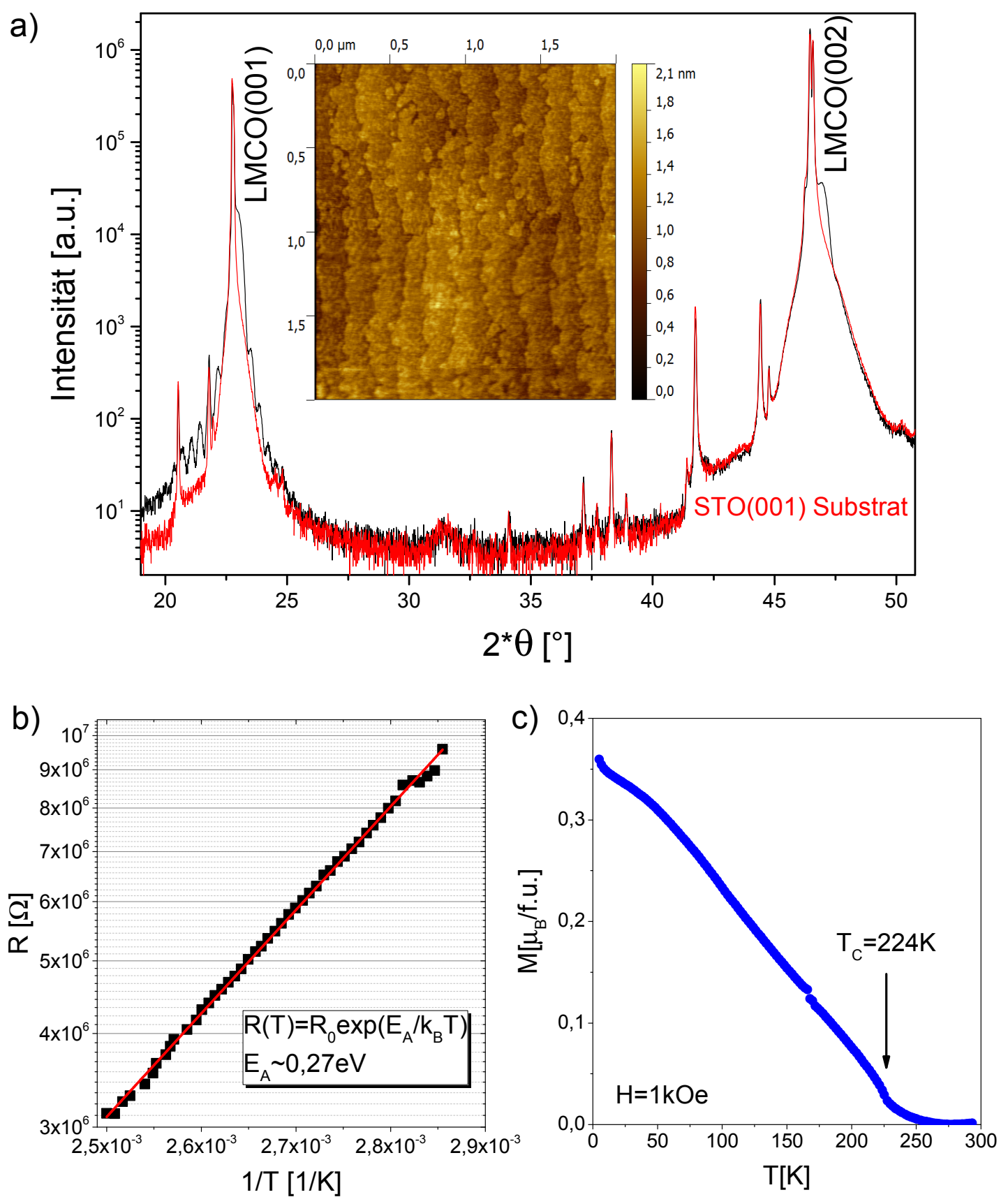

Abbildung 39: a) XRD Messung für einen 26nm LMCO Film auf STO(001) Substrat. Eine Leersubstrat-Messung ist in rot dazu gezeigt. Das eingebettete Diagramm zeigt eine $2 \times 2 \mu m^{2} S T M$ Aufnahme dieser Probe. b)Arrheniusdarstellung des elektrischen Widerstandes einer 26nm dicken LMCO Schicht auf STO(001) mit Co/Mn=1 zwischen $340-400 K$. Die Daten lassen sich linear anpassen, erhalten wird die Aktivierungsenergie von $E_{A}=0.27 \mathrm{eV}$ für den thermisch aktivierten Leitungsprozess. c) Temperaturabhängige Magnetisierung der 26nm dicken LMCO Schicht auf STO(001) bei einem externen Feld von 1kOe. Es ist ein unscharfer ferromagnetischer Übergang mit $T_{C}=224 K$ zu erkennen. 
die XRD-Messung und eine STM Aufnahme der Oberfläche eines 30nm dicken LCO Filmes auf einem STO(001) Substrat dargestellt. Neben der (00l) Phase sind keine weiteren Orientierungen oder Fremdphasen vorhanden. Die out-of-plane Gitterkonstante kann mit $c=0.377 \mathrm{~nm}$ angegeben werden, die Laue-Oszillationen zeugen von der guten kristallinen Qualität des Films. Das STM Bild zeigt atomar, glatte große Terrassen, die durch Stufen mit der Höhe von einer Gitterkonstante getrennt sind. In Abb.38 b) ist die Magnetisierung in Abhängigkeit von der Temperatur und eine $\mathrm{M}(\mathrm{H})$ Hysterese-Kurve dargestellt. Die Probe zeigt einen ferromagnetischen Übergang. Während in LCO Bulk-Kristallen die Magnetisierung bei tiefen Temperaturen $(<100 K)$, aufgrund eines Spin-Zustand-Übergangs von der high-spin $\mathrm{Co}^{3+}: t_{2 g}^{4} e_{g}^{2}$ zu low-spin $C o^{3+}: t_{2 g}^{6} e_{g}^{0}$ [139], verschwindet, existiert in tetragonal verspannten dünnen Filmen eine ferromagnetische Phase [140]. Die ferromagnetische Phase eines dünnen LCO Filmes auf $\mathrm{STO}(001)$ wurde mit $T_{C}=80-86 K$ und $M_{s}=0.3$ $0.7 \mu_{B} / C o$ charakterisiert $[141,142]$. Der MAD LCO Film zeigt ein $T_{C}=88 K$ und einen erhöhten Magnetismus von $M_{s}=1.25 \mu_{B} / C o$. Außerdem ist die out-of-plane Gitterkonstante mit $c=0.377 \mathrm{~nm}$ leicht kleiner als die Literatur Gitterkonstanten mit $c=0.378$ $0.3785 \mathrm{~nm}[141,142]$. Diese Unterschiede lassen sich auf einen unterschiedlichen Sauerstoffanteil in den Proben, verursacht durch die unterschiedlichen Depositionsverfahren (PLD und MAD), zurückführen. Mehta et al. zeigen eine Zunahme der Sättigungsmagnetisierung und der Curie-Temperatur, mit gleichzeitiger Abnahme der out-of-plane Gitterkonstante unter zunehmenden Sauerstoffpartialdruck während der Deposition [142]. Die übereinstimmenden, magnetischen Eigenschaften, die glatte Oberflächenmorphologie und die kristalline Qualität lassen auf einen nahezu stöchiometrischen LCO Film schließen. Mit diesem Ergebnis konnte angenommen werden, dass das molare $L a / C o$ - dem $L a / M n$-Verhältnis gleichgesetzt werden kann.

Die Schichtdicken von dem LCO und LMO Film lassen auf ein Co/Mn-Verhältnis von nahezu eins schließen. Die XRD-Messung eines LMCO Filmes mit den oben bestimmten $L a /(C o+M n)$ Verhältnis und gleichen, molaren Mengen von $M n$ und $C o$ ist in Abb.39 a) dargestellt. Die out-of-plane Gitterkonstante kann mit $c=0.387 \mathrm{~nm}$ angegeben werden, was im Bereich der Literaturangaben liegt [10, 136, 137]. Die Laue Oszillationen am (001)-Reflex zeugen von der guten kristallinen Qualität des Filmes. Das eingebettete Diagramm in Abb.39 a) zeigt ein STM Bild dieser Probe. Die Oberfläche ist atomar glatt und die Substratstufen zeichnen sich in der Filmoberfläche von Neuem ab. Es sind auch wenige 2d Inseln zu erkennen, was auf einen Wachstumsmodus zurück schließen lässt, der zwischen layer-by-layer- und step-flow-Wachstum liegt. Die temperaturabhängige Messung des elektrischen Widerstandes zeigt ein isolierendes Verhalten. Der Widerstand konnte mit Hilfe der DC-Option des PPMS zwischen 340 - 400K bestimmt werden, für niedrigere Temperaturen lag der Widerstand 
5 B-Platz Ordnung in ferri-/ferromagnetischen Doppelperowskiten

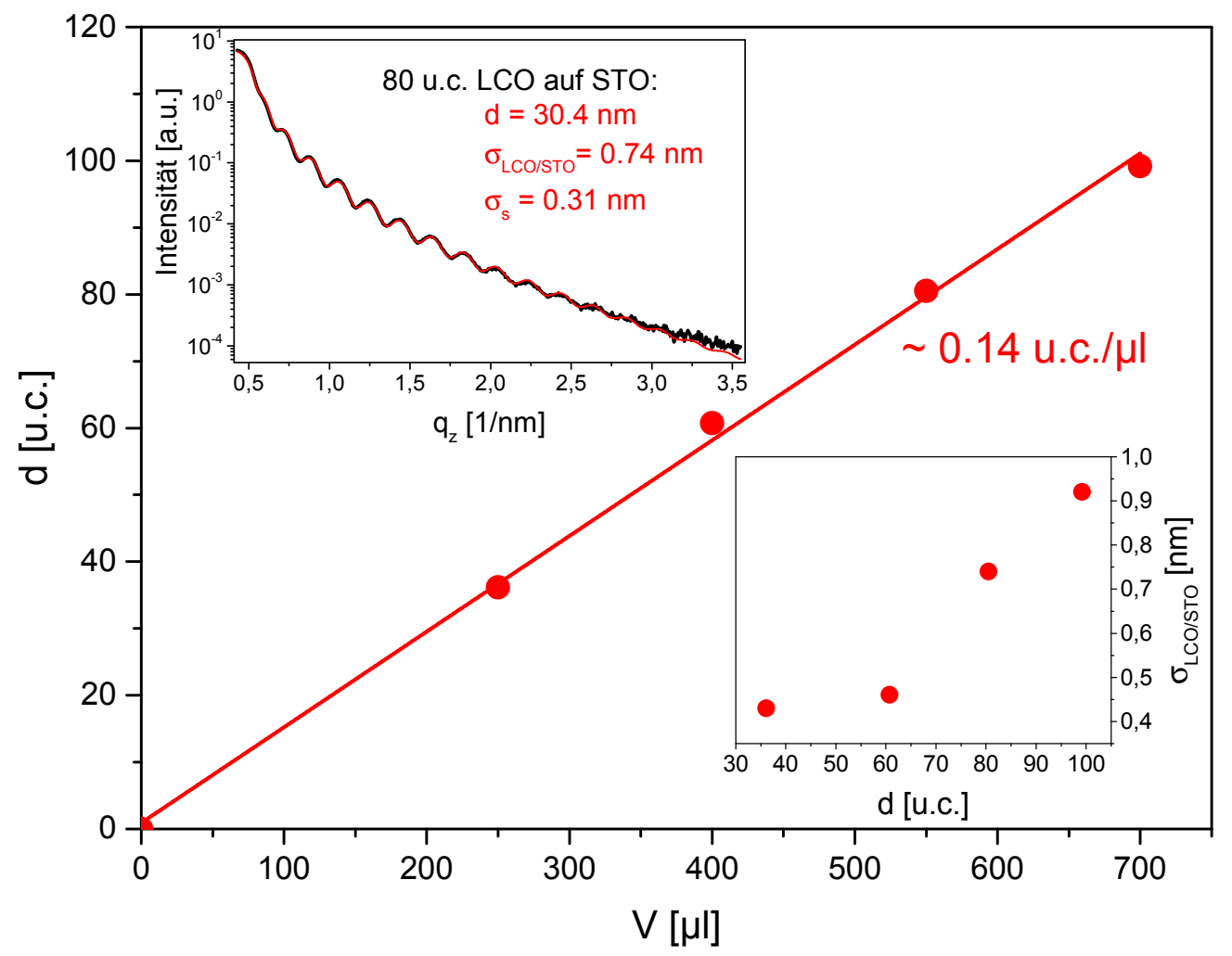

Abbildung 40: Die Schichtdicke d als Funktion des Volumen der deponierten Präkursorlösung V

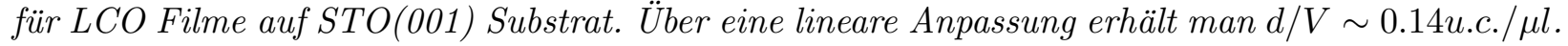
Das linke eingebettete Diagramm zeigt exemplarisch die XRR-Messungen und-Simulation für einen Film aus dieser Serie. Die Grenzfüchenrauigkeit zwischen Substrat und Film $\sigma_{L C O / S T O}$ ist im rechten eingebetteten Diagramm dargestellt.

außerhalb des Messbereichs. In der Arrheniusdarstellung lassen sich die Daten linear anpassen (siehe Abb.39b). Daraus erhält man die Aktivierungsenergie $E_{A}$ für den thermisch aktivierten Leitungsprozess. Diese stimmt mit $E_{A}=0.27 \mathrm{eV}$ der Aktivierungsenergie in PLD Filmen überein [136]. Die Magnetisierung in Abhängigkeit von der Temperatur offenbart bereits eine Curie-Temperatur von $224 K$ nahe dem $T_{C}$ der geordneten Phase, jedoch ist der Übergang sehr unscharf und die Magnetisierung klein (siehe Abb.39 c). Obwohl ein Teil der gewünschten, geordneten LMCO Phase bereits vorhanden ist, gibt es in diesem System einen weiteren Optimierungsbedarf.

Um die Stöchiometrie (und damit möglicherweise die magnetischen Eigenschaften) der folgenden LMCO Schichten zu verbessern, wurde das molare $C o / M n$-Verhältnis mit Hilfe einer Schichtdickenserie von LCO und dem, bereits aus Kap. 4.2 bekannten, $d / V$-Verhältnis für Manganate bestimmt. Die Schichtdicke $d$, die RMS-Rauigkeiten der Oberfläche $\sigma_{s}$ und der Grenzfläche zwischen Film und Substrat $\sigma_{L C O / S T O}$ der LCO Filme wurde anhand von Simulationen von XRR-Messungen durchgeführt (siehe eingebettetes Diagramm in Abb.40). Die 
Simulationen wurden mit der Software ReMagX durchgeführt [51]. In Fig.40 ist die Schichtdicke $d$ und in Abhängigkeit vom deponierten Volumen der Präkursorlösung $V$ gezeigt. Für LCO ergibt sich ein $d / V$ Verhältnis von 0.14u.c./ $\mu l$. Während sich das quadratische Mittel der Oberflächenrauigkeit unabhängig von der Schichtdicke zwischen $\sigma_{s} \sim 0.2-0.4 \mathrm{~nm}$ bewegt, steigt die Grenzflächenrauigkeit $\sigma_{L C O / S T O}$ monoton mit der Schichtdicke $d$ an (siehe eingebettetes Diagramm in Abb.40). Diese Werte liegen im Bereich der Grenzflächenrauigkeiten von LSMO. Führt man dieses Ergebnis mit den $d / V$-Verhältnissen für Manganate aus Kap. 4.2 zusammen, kann das molare $C o / M n$ Verhältnis in der Lösung, um eine Stöchiometrie von $C o / M n=1 / 1$ in dem Film zu erhalten, zu Co/Mn $=0.925$ bestimmt werden. Wenn des Weiteren von dem $C o / M n$ Verhältnis gesprochen wird, ist damit das molare $C o / M n$ Verhältnis $\rho$ im Film gemeint.

\subsubsection{Strukturelle und magnetische Eigenschaften von dicken $\mathrm{La}_{2} \mathrm{CoMnO}_{6}$ Schichten auf STO(111)}

An dieser Stelle ist anzumerken, dass die Co-Mn-Kationen-Ordnung in der Literatur bisher vorrangig über die magnetischen Eigenschaften der Proben bestimmt wurde. Dies ist jedoch nur eine globale Messmethode für die Nahordnung. In dieser Arbeit wurde beabsichtigt die B-Platz-Ordnung auch über XRD und TEM zu bestimmen. Aus diesem Grund wurden die LMCO Filme vorrangig auf (111) orientierten STO Substraten gewachsen. Wie man in Abb.41 sehen kann, sind die (111) Ebenen abwechselnd von Mn oder Co besetzt. Daraus resultiert eine Überstruktur, die in XRD und TEM Messungen beobachtet und die Ordnung quantifiziert werden kann. Da sich die atomaren Formfaktoren von $M n$ und Co nur minimal unterscheiden [46], besitzt der Überstrukturreflex im XRD nur eine sehr kleine Intensität. Aus diesem Grund wurden dicke LMCO Schichten mit einer Schichtdicke von bis zu 325nm gewachsen. Eine Schichtdicke dieser Größe kann nicht mehr mit Hilfe der Röntgenreflektometrie aufgelöst werden, sie wird jedoch später in diesem Kapitel in den TEM Aufnahmen bestätigt.

Aufgrund der Beobachtung von Goodenough et al. [10], dass das Auftreten von $M n^{3+}$-Ionen in LMCO zu einer Störung der Ordnung führt, wurde das Co/Mn Verhältnis $\rho$ in den, im Folgenden betrachteten LMCO Filmen, in die Richtung eines höheren $C o$-Anteils verschoben $(\rho \geq 1)$. Abb.42 a) zeigt die XRD-Messung von einem LMCO Film auf STO(111) Substrat mit $\rho=1.075$ und einer Schichtdicke von $d \sim 325 \mathrm{~nm}$. Der pseudokubische (111) Reflex wie auch das Substrat zeigt, eine Aufspaltung in zwei Reflexe, verursacht durch die zwei $C u_{\alpha}$-Wellenlängen des in diesem Experiment verwendeten Röntgenlichtes $\left(\alpha_{1}-\alpha_{2}\right.$ - 

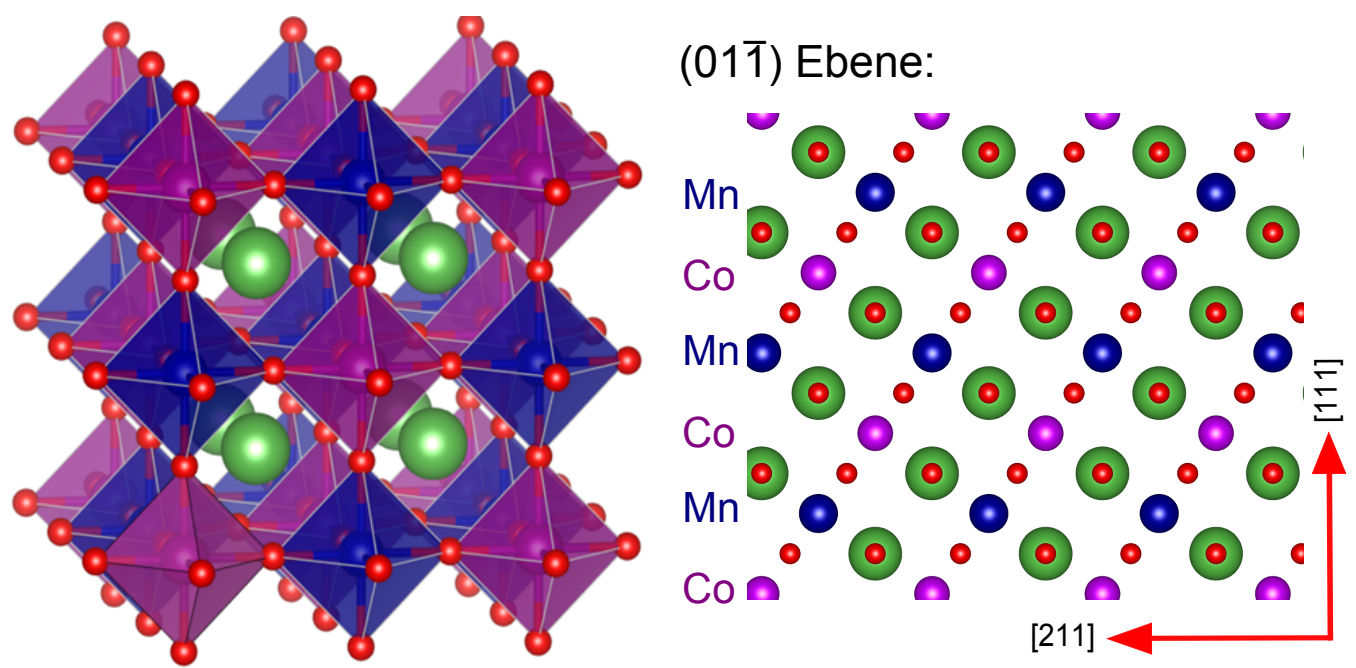

Abbildung 41: Links: Ideal geordnete Doppelperowskitstruktur: Mn(blau) und Co(violett) sind von einem Sauerstoffoktaeder(rot) umgeben. Co und Mn ordnen sich in einer Kochsalzstruktur an. Auf dem A-Platz ist das La(grün) zu finden. Rechts: Die gleiche Struktur wie links als Projektion in der (011) Ebene. Die (111) Ebenen sind abwechselnd nur von Mn und Co besetzt. Entlang der [111] Richtung kann die Überstruktur beobachtet werden.

Aufspaltung). Um diese Aufspaltung beobachten zu können, muss die Halbwertsbreite des Reflexes klein sein $\left(q_{z}<0.07 / \mathrm{nm}\right)$. Daraus folgt, dass der Film zum einen relativ dick $(>100-200 \mathrm{~nm})$ sein muss, zum anderen muss er eine gute kristalline Qualität aufweisen (Scherrer-Gleichung [143]). Neben dem (111) Reflex, sind auch die Reflexe der Überstruktur zu beobachten: Bei $2 \cdot \theta=19.9^{\circ}$ ist der $(1 / 21 / 21 / 2)$ - und bei $2 \cdot \theta=62.3^{\circ}$ der $(3 / 23 / 2$ $3 / 2$ )-Reflex zu sehen (eingebettetes Diagramm in Abb.42 a). Letzterer ist wiederum $\alpha_{1}$ $\alpha_{2}$ aufgespalten. Zusätzlich ist bei $2 \cdot \theta=36.5^{\circ}$ ein Fremdphasenreflex zu sehen, dem $\mathrm{CoO}$ oder auch $\mathrm{CoMnO}_{3}$ zugeordnet werden kann. Um einen quantitativen Wert der B-Platz Ordnung, die mit Hilfe der Röntgendiffraktometrie bestimmt wurde, zu erhalten, kann man einen XRD-Ordnungs-Parameter folgendermaßen definieren: $S=I(1 / 21 / 21 / 2) / I(111)$. Dabei ist $I(h k l)$ die entsprechenden Intensität des $(h k l)$ Reflexes nach Abzug des SubstratHintergrundes. Für diese Probe erhält man $S(\rho=1.075)=1.1 \cdot 10^{-3}$. Die Magnetisierungsdaten dieser Probe sind in Abb.42 b) dargestellt. Bei einem externen Feld von $1 k O e$ ist ein ferromagnetischer Übergang bei $T_{C}=226 K \mathrm{zu}$ erkennen. Die $M(H)$-Hystererkurve bei $5 K$ zeigt eine Sättigungsmagnetisierung von $M_{s}(50 k O e)=5.8 \mu_{B} / f$.u. bei hohen externen Feldern. Diese Werte stimmen gut mit den Literaturwerten für geordnete Bulk-Kristalle und Filme überein [10, 135-138].

Eine Zusammenfassung der Daten von LMCO Schichten mit verschiedenen $C o / M n$-Verhältnissen $\rho=0.99$ - 1.18 und einer Schichtdicke von 325nm ist in Abb.43 präsentiert. Die pseudokubische Gitterkonstante $c$ steigt mit zunehmendem $C o$-Gehalt an. Gleichzeitig bleibt 

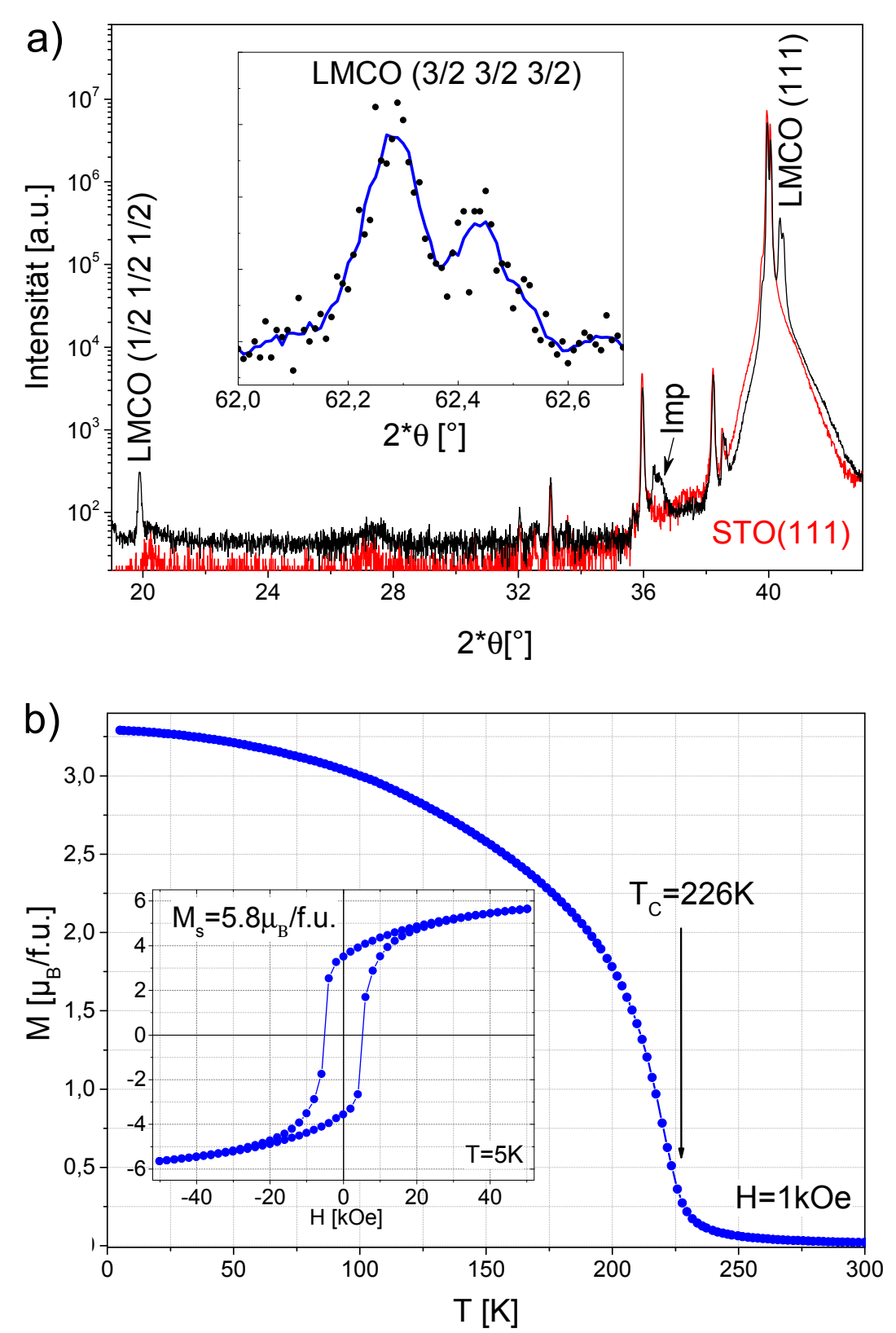

Abbildung 42: a) XRD-Daten eines 325nm dicken LMCO Films auf STO(111) mit einem Co/Mn Verhältnis $\rho=1.075$ (schwarz). Eine Leersubstrat-Messung ist in rot gezeigt. Man erkennt die Überstrukturreflexe (1/2 1/2 1/2) und (3/2 3/2 3/2) (eingebettetes Diagramm, die blaue Linie zeigt die geglätteten Daten) neben dem pseudokubischen Perowskit-Reflex (111). Ein auftretender Fremdphasenreflex ist mit "Imp" gekennzeichnet. b) Magnetisierung $M$ in Abhängigkeit von der Temperatur $T$ desselben Films bei einem angelegten externen Feld von $H=1 k O e$. Das eingebettete Diagramm zeigt die $M(H)$ Hysterese Kurve nach dem Feldeinkühlen bei $1 k O e$ auf $5 K$. 


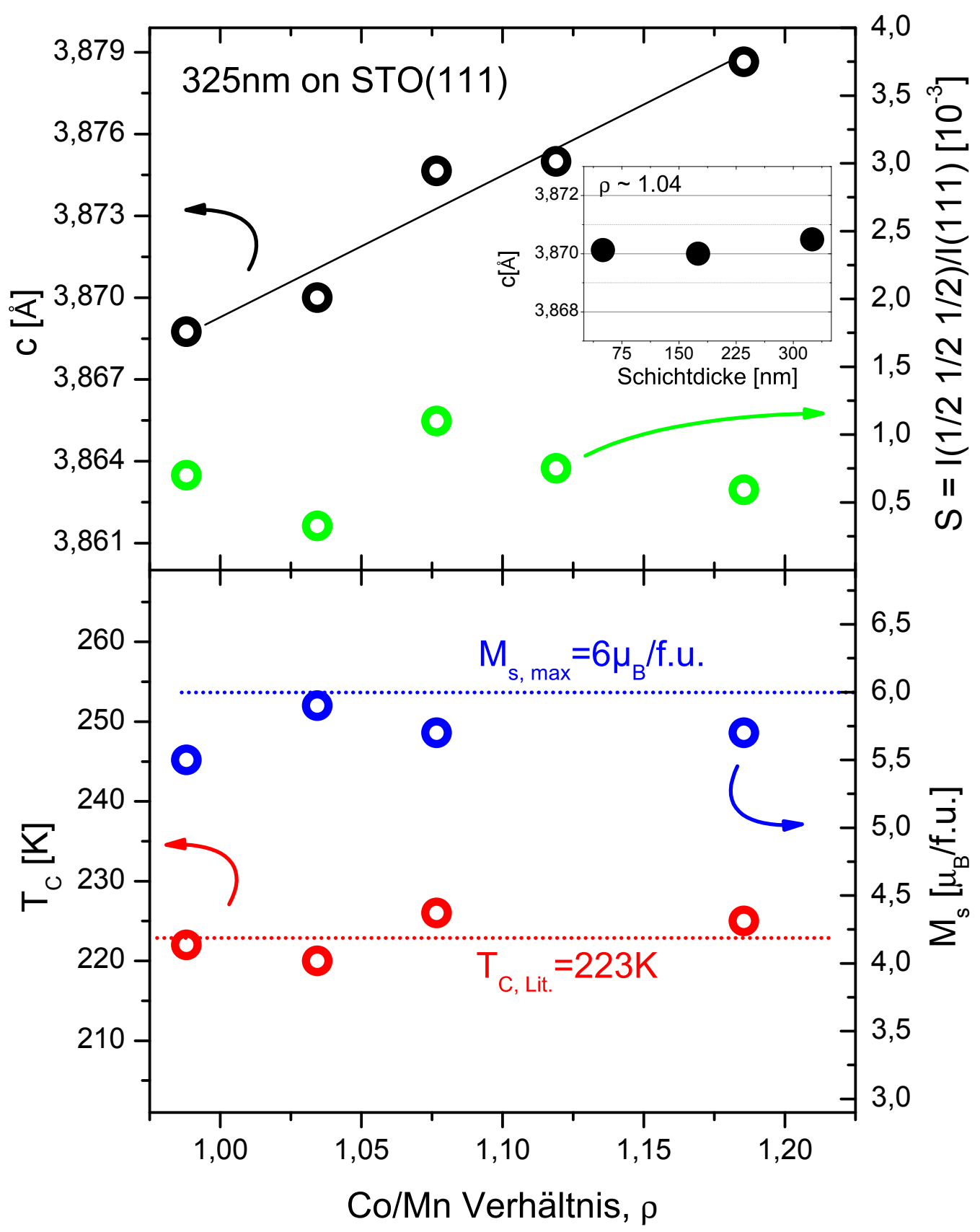

Abbildung 43: Pseudokubische Gitterkonstante c (schwarz, die Gerade dient als optische Führung), XRD-Ordnungs-Parameter $S$ (grün), Sättigungsmagnetisierung $M_{s}$ (blau), und CurieTemperatur $T_{C}$ (rot) von LMCO Filmen mit einer Schichtdicke von 325nm als Funktion des Co/Mn-Verhältnisses $\rho$. Der Literaturwert für $T_{C, \text { Lit. }}$ (rote gepunktete Linie) und $M_{s, \max }$ (blaue gepunktete Linie) für geordnetes LMCO Material ist aus [10, 135-138] entnommen. Das eingebettete Diagramm zeigt die Gitterkonstante c als Funktion der Schichtdicke für LMCO Filme mit $\rho=1.04$. 


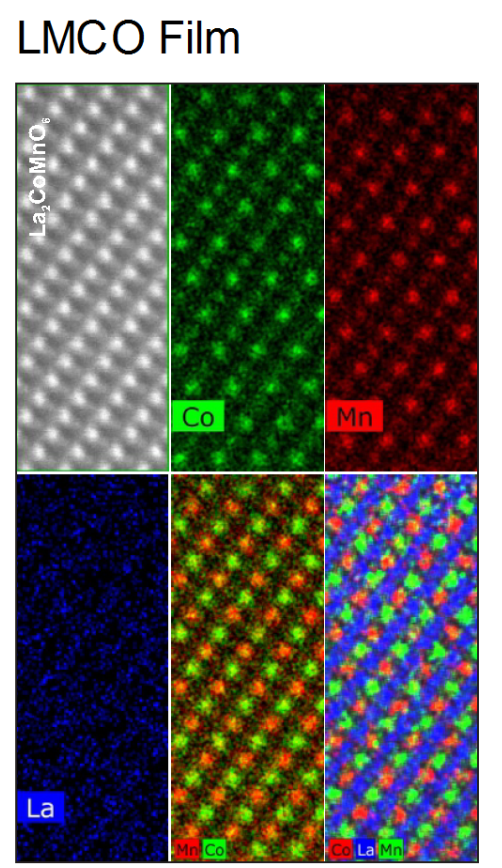

LMCO/STO Grenzfläche

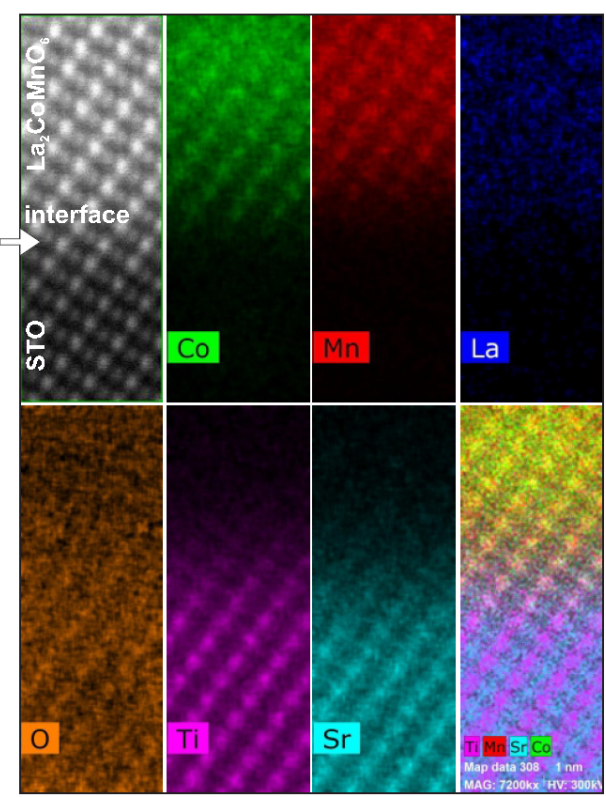

Abbildung 44: HAADF Aufnahme und korrespondierende EDX elementspezifische Karten an der LMCO/STO Grenzfläche (rechts) und weit entfernt vom Substrat (links). Die kombinierten Elementkarten mit Co (grün), $M n$ (rot), La (blau), Sr (cyan), Ti (magenta) und O (orange) demonstrieren die Anwesenheit von B-Platz Nahordnung im LMCO Film und die Abwesenheit dieser Ordnung nahe dem Substrat. Adaptiert aus der Literatur [53].

die Intensität des Fremdphasen-Reflexes nahezu konstant. Daraus kann man vorerst schließen, dass die angestrebte kompositionelle Änderung der Filmstöchiometrie tatsächlich übernommen wurde. Zusätzlich ist die Gitterkonstante nicht von der Schichtdicke abhängig, wie man in dem eingebetteten Diagramm von Abb.43 sehen kann, indem die Gitterkonstante für verschiedene Schichtdicken von LMCO Filmen mit $\rho=1.04$ gezeigt sind. Der XRDOrdnungs-Parameter $S$ bewegt sich ohne erkennbares Muster in einem Bereich zwischen $0.35 \cdot 10^{-3}$ und $1.1 \cdot 10^{-3}$ für alle Proben. Auch die magnetischen Daten zeigen bemerkenswerterweise nahezu keine Veränderung und kein Muster bei der Variation von $\rho$ : Die Sättigungsmagnetisierung $M_{s}$ liegt zwischen 5.5 - $5.9 \mu_{B} / f$.u., die Curie-Temperatur $T_{C}$ zwischen 220 - 226K. Damit liegt $T_{C}$ aller betrachteten Proben um den mittleren Literaturwert (rote gepunktete Linie in Abb.43) und die Sättigungsmagnetisierung aller betrachteten Proben nahe dem maximalen theoretischen $M_{s, \max }=6 \mu_{B} / f . u$. (blaue gepunktete Linie in Abb.43) für das B-Platz geordnete LMCO Material.

An dem LMCO Film mit dem größten XRD-Ordnungs-Parameter $S(\rho=1.07)=1.1 \cdot 10^{-3}$ wurden lokale, strukturelle und elektrische Untersuchungen mittels TEM in der Gruppe um J. Verbeeck am EMAT der Universität von Antwerpen vorgenommen. Auf die technischen Details dieser Messungen und die Auswertung dieser Daten wird genauer in der Dissertation 


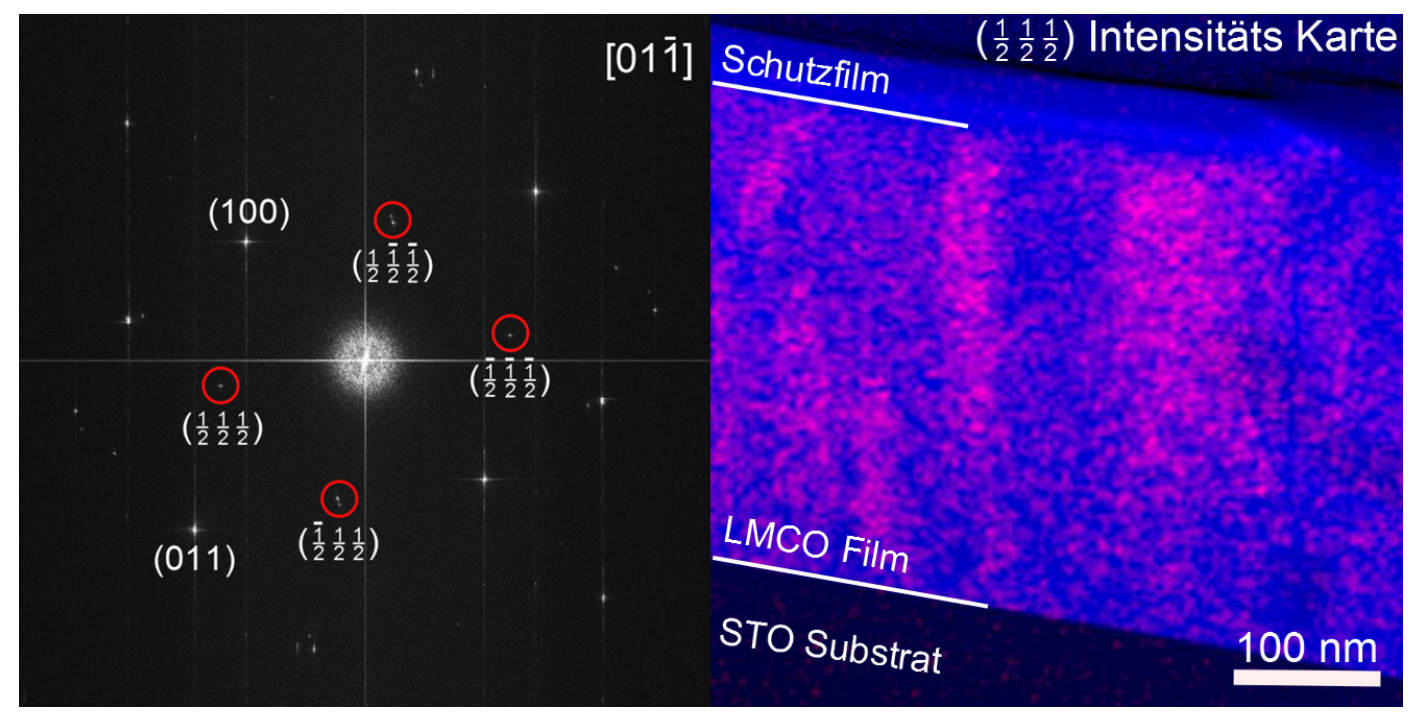

Abbildung 45: Links: 2d-Fouriertransformation einer hochaufgelösten HAADF Aufnahme. Die Überstrukturreflexe sind mit roten Kreisen markiert. Rechts: Zurücktransformation des (1/2 1/2 1/2)-Reflexes in den Ortsraum. Man erkennt verschiedene Bereiche mit höheren (pink) und niedrigeren Überstrukturintensitäten (blau).

von Ricardo Egoavil eingegangen [53].

Wie in Abb.44 zu sehen ist, konnte die B-Platz Nahordnung lokal durch ortsaufgelöste EDX Karten bestätigt werden. In Abb.44 sind die einzelnen Elementkarten für die $L a L_{\alpha}, C o K_{\alpha}$, $M n K_{\alpha}, \operatorname{Sr} L_{\alpha}$, Ti $K_{\alpha}$ und $O K_{\alpha}$ für einen Bereich nahe dem Substrat (rechts) und weit weg von dem Substrat (links) dargestellt. Dabei ist die Projektionsebene, wie in der Skizze in

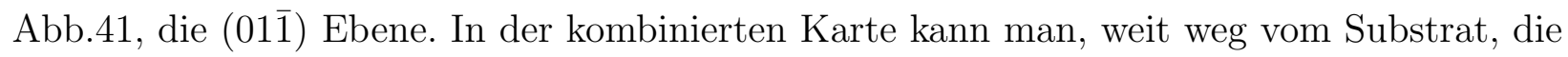
aus der B-Platz Ordnung resultierenden Überstruktur entlang der [111] Richtung erkennen. Nahe dem Substrat ist diese Ordnung nicht vorhanden. Auch das epitaktische Wachstum des Films sowie eine Interdiffusionzone zwischen Substrat und Film mit einer Größe von $\sim 1 n m$ sind hier zu sehen.

Um die Ordnung auf einer größeren Skala auflösen zu können, wurden hochaufgelöste HAADF Aufnahmen mit einer Größe von $460 \times 460 \mathrm{~nm}^{2}$ und einer Auflösung von $8192 \times 8192$ Bildpunkten erstellt. Aufgrund des geringen $Z$-Unterschiedes zwischen $C o$ und $M n$, müssen diese Bilder mit einer Fourierfilter-Methode analysiert werden [53]: Die 2d-Fouriertransformation einer solchen HAADF-STEM Aufnahme von dem LMCO Film ist auf der linken Seite von Abb.45 zu sehen. Die Überstrukturreflexe der $\{1 / 21 / 21 / 2\}$-Ebenen sind klar zu erkennen (im Bild markiert durch rote Kreise). Transformiert man einen dieser Überstrukturreflexe zurück in den Ortsraum, erhält man das Bild auf der rechten Seite von Abb.45. Dabei ist der entstandene Kontrast ein Maß für die Intensität des Überstrukturreflexes und damit ein Maß für die B-Platz Ordnung. In der Karte erkennt man ortsabhängige Instensitätsvariationen 

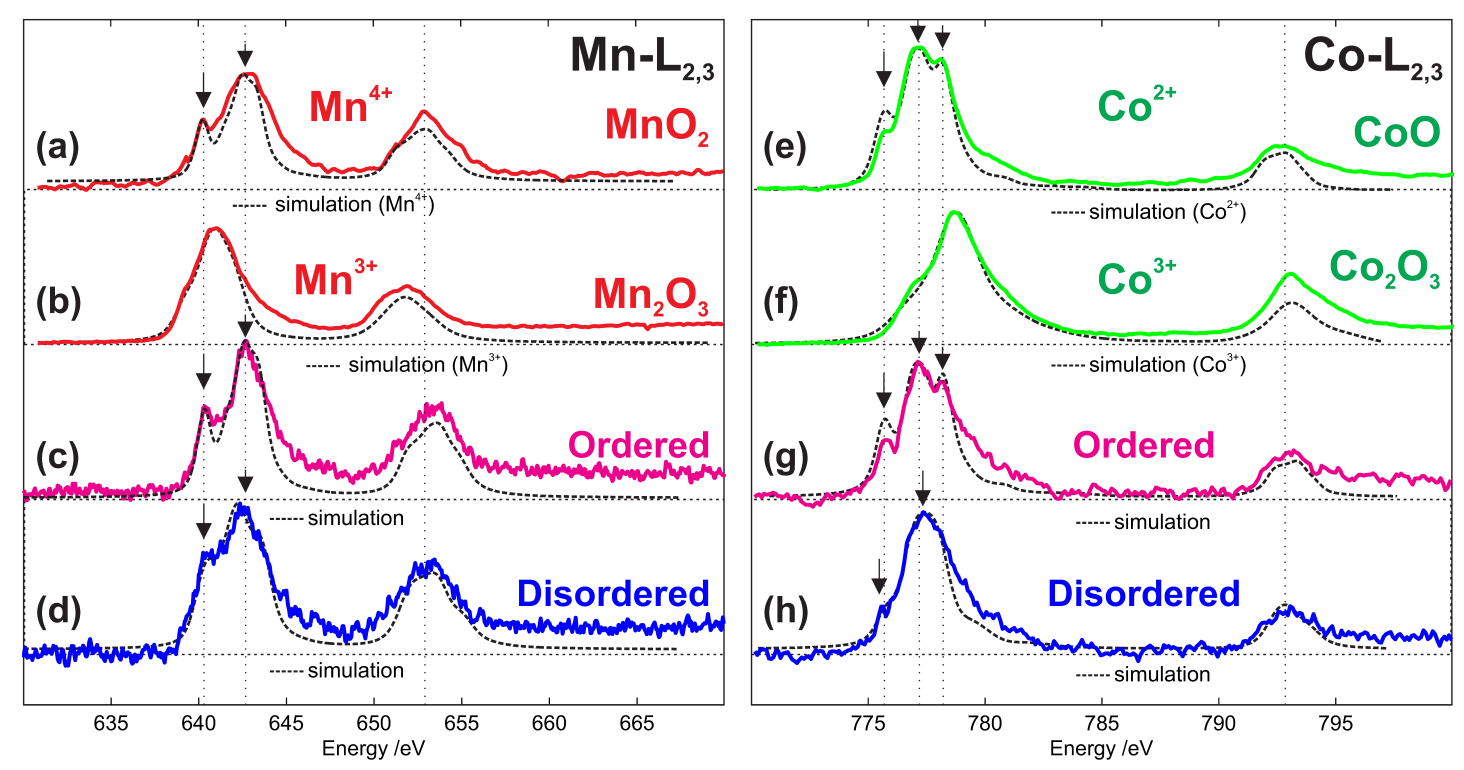

Abbildung 46: Gezeigt sind EELS-Spektren von B-Platz Kationen in LMCO: (a,b und e,f) Spektrum der $\mathrm{Mn}^{4+}\left(\mathrm{MnO}_{2}\right), \mathrm{Mn}^{3+}\left(\mathrm{Mn}_{2} \mathrm{O}_{3}\right), \mathrm{Co}^{2+}(\mathrm{CoO})$ und $\mathrm{Co}^{3+}\left(\mathrm{Co}_{2} \mathrm{O}_{3}\right)-\mathrm{L}_{2,3}$ Kante. (c, d und g,h) Spektren der $M n-L_{2,3}$ und $C o-L_{2,3}$ Kante von Bereichen mit höheren (pink) und niedrigeren Überstruktur-Intensitäten (blau) (siehe Abb.45). Aus Literatur [53] entnommen.

des (1/2 1/2 1/2)-Reflexes. Die Bereiche mit einer höheren Intensität/Ordnung sind dabei pink, Bereiche mit einer niedrigeren Intensität/Ordnung blau eingefärbt. Das der metallische Schutzfilm, der auf dem TEM Filament prepariert wurde scheinbar ein schwaches $(1 / 21 / 2$ 1/2) Signal liefert, ist dabei ein Artefakt der Nachbearbeitung [53]. Bemerkenswerterweise ist die $(1 / 21 / 21 / 2)$ Intensität in dem LMCO Film an jedem Ort größer null, es ist daher immer eine gewisse Nahordnung vorhanden.

Die elektronische Struktur von $M n$ und $C o$, sowohl in Bereichen mit höherer Ordnung als auch niedrigerer Ordnung, wurde mit der Elektronenenergieverlustspektroskopie (EELS) untersucht. Die Ergebnisse sind in Abb.46 dargestellt. Zunächst ist festzustellen, dass sich die hochaufgelösten EELS Spektren, sowohl der $M n-L_{2,3}$ als auch der $C o-L_{2,3}$-Kante, aus den Bereichen höherer und niedrigerer Ordnung sehr ähneln. Im Vergleich mit den Spektren der $\mathrm{Mn}^{4+}\left(\mathrm{MnO}_{2}\right), \mathrm{Mn}^{3+}\left(\mathrm{Mn}_{2} \mathrm{O}_{3}\right), \mathrm{Co}^{2+}(\mathrm{CoO})$ und $\mathrm{Co}^{3+}\left(\mathrm{Co}_{2} \mathrm{O}_{3}\right)$ Oxiden, zeigt sich eine große Übereinstimmung der LMCO $M n-L_{2,3}$ und $C o-L_{2,3}$ Spektren in dem Bereich mit größere Überstruktur-Intensität, in Bezug auf die Peak-Position (in Abb.46 angedeutet durch die Pfeile) sowie die Form der Kurve, mit $M n^{4+}$ beziehungsweise $C o^{2+}$. Auch die $M n$ und $C o$ Spektren aus dem Bereich mit niedrigerer Ordnung können vornehmlich diesen Valenzen zugeordnet werden, jedoch ist zum einen der $M n-L_{3}$ leicht verbreitert und dem Co-Spektrum fehlt der Peak bei 777.1eV im Vergleich zu den Spektren aus den Bereichen höherer Ordnung. Dies deutet darauf hin, dass sich die Valenz von Co zwischen $2+$ und $3+$ und für $M n$ zwi- 


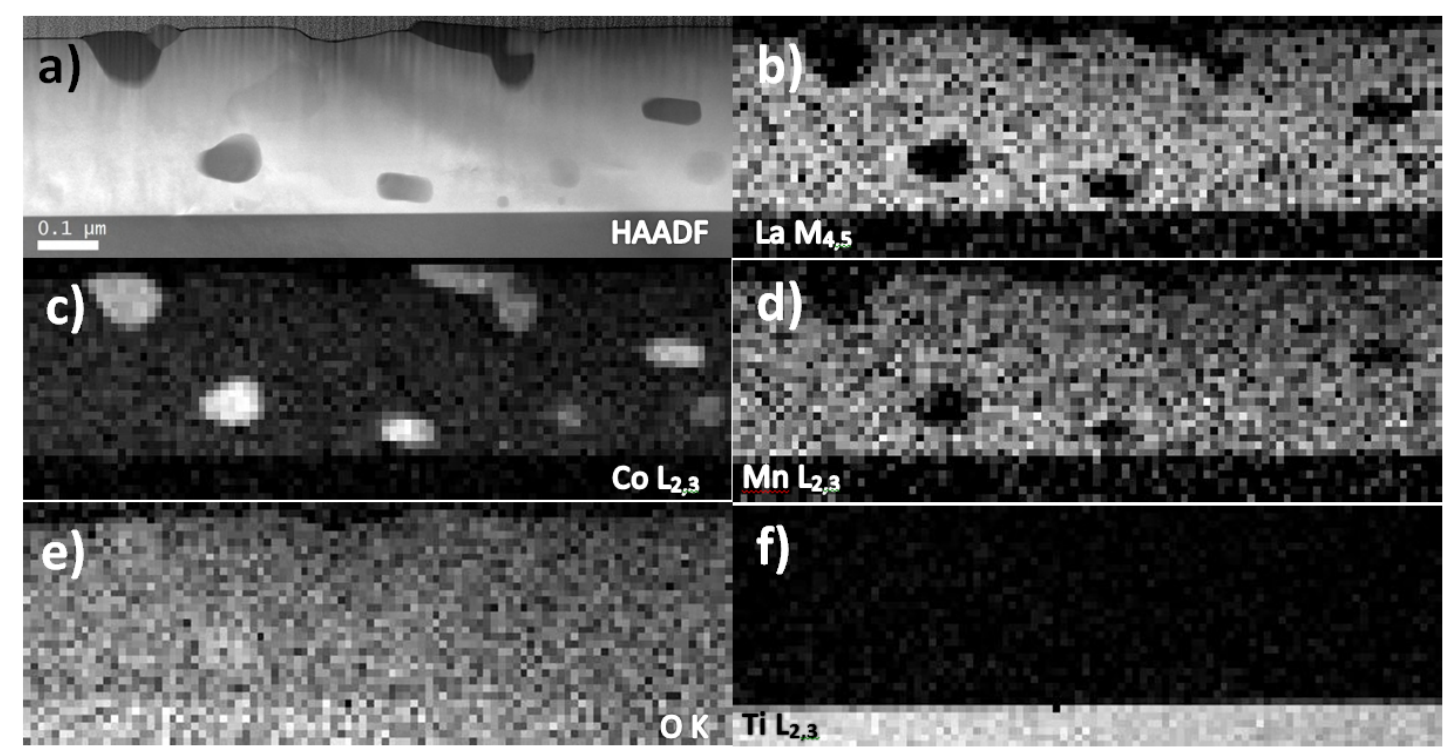

Abbildung 47: a) In der HAADF Aufnahme von LMCO auf großer Skala sind dunklere Bereiche innerhalb des Films vorhanden. b) to f) EELS elementspezifische Karten der La - $M_{4,5}, O-K$, Co - $L_{2,3}, M n-L_{2,3}$ und $T i-L_{2,3}$ Kanten, wie angegeben.

schen $3+$ und $4+$ bewegt. Mit Hilfe einer Linearkombination der $\mathrm{Mn}^{4+}-\mathrm{Mn}^{3+}$ - und $^{\mathrm{Co}^{2+}}{ }_{-}$, $\mathrm{Co}^{3+}$-Oxid Spektren kann der Anteil des Volumens des betrachteten Bereiches der LMCO Probe mit der Valenzkonfiguration $\left(\mathrm{Co}^{2+}, \mathrm{Mn}^{4+}\right)$ bestimmt werden: in den Bereichen höherer Ordnung beträgt diese $(99 \%, 97 \%)$, in den Bereichen niedrigerer Ordnung (90\%,77\%) [53]. Das diese beiden Bereiche bevorzugt eine $\left(\mathrm{Co}^{2+}, \mathrm{Mn}^{4+}\right)$-Valenzkonfiguration zeigen, wird nochmals durch Multiplet-Simulationen von einzelnen $\mathrm{Co}^{2+}$ und $\mathrm{Mn}^{4+}$ Zuständen in einer oktaedrischen Umgebung bestätigt [53]. Das Ergebnis der Simulationen ist in jedem Spektrum in Abb.46 durch die gestrichelte Linie gezeigt.

Wie in Abb.47 a) zu sehen ist, lassen sich in großskaligen HAADF Aufnahmen dieser LMCO Probe dunklere Bereiche erkennen. Korrespondierende, elementaufgelöste EELS Karten (Abb.47 b-f) zeigen die Abwesenheit von $L a$ in all diesen Bereichen. Es existiert ein $L a$-Defizit in dem Film, welches sich ungleichmäßig verteilt. Zusätzlich gibt es in manchen Bereichen auch einen Mangel an $M n$ und einen Überschuß an Co. Dies kann mit dem Co/MnVerhältnis $\rho=1.07$ in Verbindung gebracht werden. Zusammenfassend besteht der Film größtenteils aus stöchiometrisch richtigem LMCO und die stöchiometrischen Fehler agglomerieren in Ausscheidungen von $\mathrm{Co}_{2-x} \mathrm{Mn}_{x} \mathrm{O}_{3}$ oder $\mathrm{Co}_{3-x} \mathrm{Mn}_{x} \mathrm{O}_{4}$ Material. Das La-Defizit wurde durch ein erneutes Einstellen des $L a / M n$-Verhältnisses mittels der Präparation von LMO auf eine Größe von 3 - 4\% bestimmt. Möglicherweise hat sich der La-Präkursor leicht geändert, oder das $L a /(M n+C o)$-Verhältnis ist nicht mit dem $L a / M n$-Verhältnis gleich zu setzen. Aus den EELS Karten (Abb.47) lässt sich ein La-Defizit von 5 - $10 \%$ abschätzen. 
Die Diskrepanz zu dem, aus der Präparation bestimmten Wert, lässt sich darauf zurück führen, dass TEM nur eine lokale Messmethode ist: HAADF Bilder anderer Bereiche der gleichen Probe zeigten eine geringe Anzahl von Defekten, die auf $L a$-Defizit zurückzuführen sind (vergleiche mit Abb.45).

Aus diesem Resultat folgend, wurde noch ein 290nm LMCO Film mit $\rho=1.07$ und dem angepassten $L a / M n$-Verhältnis auf STO(111) gewachsen. Die Charkterisierungs-Daten sind in Abb.48 aufgeführt. Dieser Film zeigt nur einen sehr schwachen (1/2 1/2 1/2) Überstrukturreflex (schwarz in Abb.48 a) und zwei verschiedene LMCO Phasen in der XRD Messung (schwarz in Abb.48 b): Die pseudokubischen Gitterkonstanten können auf $c_{1}=0.387 \mathrm{~nm}$ und $c_{2}=0.383 \mathrm{~nm}$ bestimmt werden, beide sind kleiner als die pseudokubische Gitterkonstante des $L a$-defizitären Filmes mit $\rho=1.07$. In der temperaturabhängige Magnetisierung (schwarz in Abb.48 c) sind auch zwei verschiedene ferromagnetische Phasen zu erkennen, mit den Curie-Temperaturen $T_{C 1}=223 \mathrm{~K}$ und $T_{C 2}=131 \mathrm{~K}$. Auch die Sättingungsmagnetisierung bei tiefen Temperaturen ist mit $2.2 \mu_{B} / f$. u viel kleiner als in allen $L a$-defizitären Proben. Im Vergleich zu den Literaturergebnissen lassen sich alle diese Daten zusammenfassend deuten, dass dieser Film aus einer B-Platz-geordneten und einer B-Platz-ungeordneten Phase besteht. Tatsächlich ist das Volumen der Einheitszelle für das ungeordnete LMCO, mit einer Curie-Temperatur von $T_{C} \sim 130 K$, kleiner als das Volumen der Einheitszelle der geordneten Phase $[10,135,136]$. Eine B-Platz Ordnung konnte in dem Film nach einer zwölf stündigen thermischen Auslagerung in Luft bei $900^{\circ} \mathrm{C}$ erzeugt werden: Der Überstrukturreflex hat sich auf einen Wert von $S=6.5 \cdot 10^{-4}$ erhöht und ist somit im Bereich der geordneten La-defizitären Proben (rot in Abb.48 a). Es ist ausschließlich eine Phase mit einer pseudokubischen Gitterkonstante von 0.3876nm und einer Curie-Temperatur von $T_{C}=227 \mathrm{~K}$ vorhanden (rot in Abb.48 b und c). Auch die Sättigungsmagnetisierung hat sich auf einen Wert von $5.4 \mu_{B} / f$.u. erhöht (rot in Abb.48 d). Im Vergleich dazu führt die thermische Auslagerung der $L a$-defizitären Proben zu keiner Änderung der magnetischen Eigenschaften und zu einer arbiträren leichten Änderung des Überstrukturreflexes.

\subsubsection{Diskussion der B-Platz Ordnung in $\mathrm{La}_{2} \mathrm{CoMnO}_{6}$ Filmen}

Die Größe der Co/Mn Nahordnung, definiert durch die Wahrscheinlichkeit $0<p<1$, dass ein $\mathrm{Mn}$-Ion ein $\mathrm{Co}$-Ion als nächsten B-Platz Nachbarn hat, kann durch die magnetischen Eigenschaften indirekt abgeschätzt werden. In Anbetracht des theoretischen Wertes von $M_{s}=6 \mu_{B} / f . u$. in einem vollständig geordneten $L a_{2} C o^{2+} M n^{4+} O_{6}$-System mit $p=1$, kann die Nahordnung für alle betrachteten LMCO Filme auf STO(111) auf $p=0.92$ - 
5 B-Platz Ordnung in ferri-/ferromagnetischen Doppelperowskiten
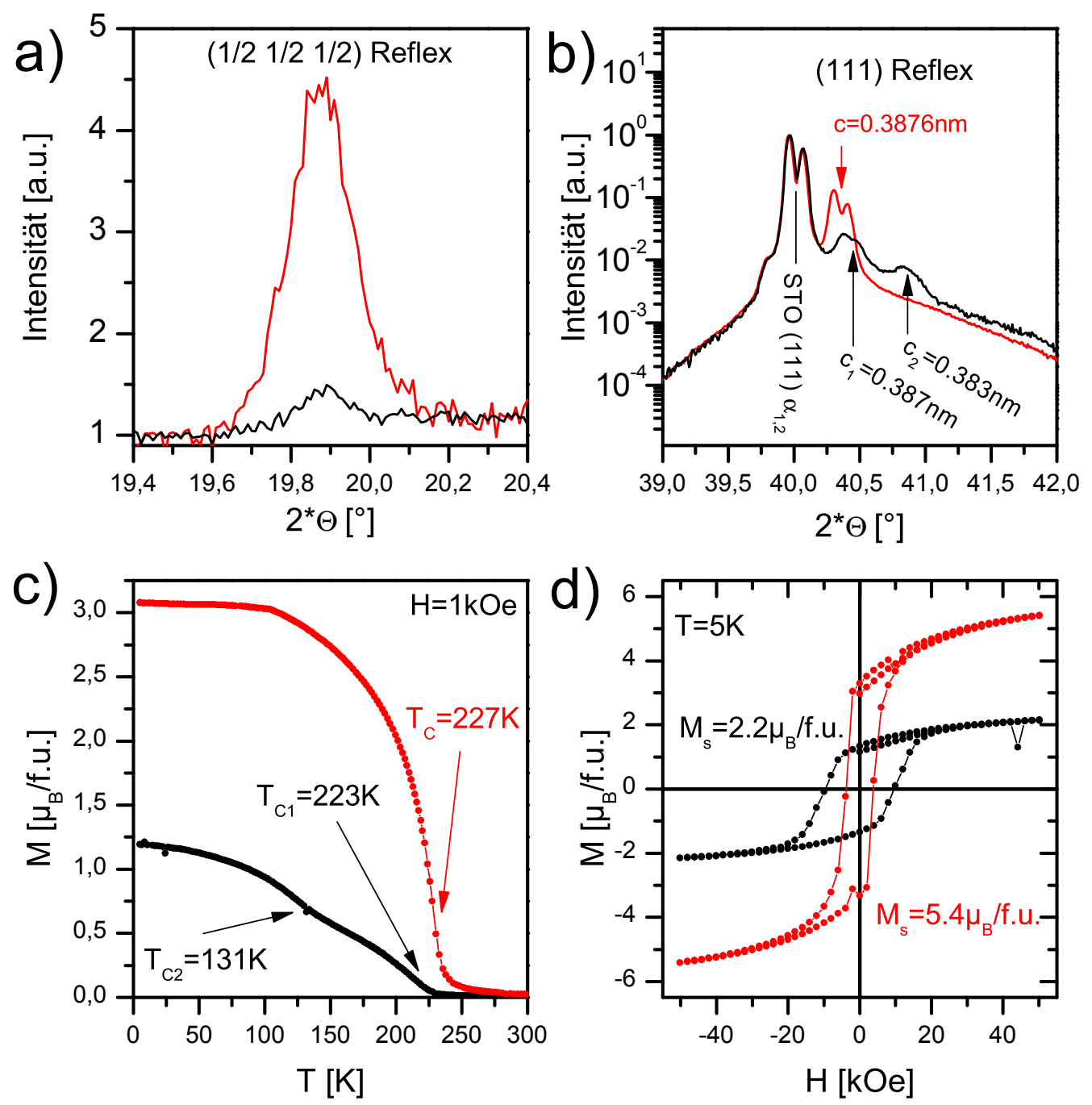

Abbildung 48: Resultate der Charakterisierung eines 290nm LMCO mit $\rho=1.07$ ohne La-Defizit auf STO(111). Es sind die Daten vor der thermischen Auslagerung in schwarz und danach in rot gezeigt. a) und b) zeigt die XRD Messungen am (1/2 1/2 1/2)- und (111)-Reflex. c) Die temperaturabhängige Magnetisierung bei einem externen Feld von $1 k O e$. d) Die M(H) HystereseKurve nach dem Feldeinkühlen bei 1 kOe auf $5 K$. 
0.98 abgeschätzt werden. Aus dem Verlauf der $M(H)$ Kurven kann jedoch geschlossen werden, dass die LMCO Filme bei $5 T$ noch nicht vollständig gesättigt sind. Das bedeutet in den Filmen sind maximal $2-8 \%$ der $B / B^{\prime}$-Paare, Co/Co- oder $M n / M n$-Paare. Diese Werte sind wie die Magnetisierungs-Daten, aus denen sie resultieren, mit ca. $5 \%$ Fehler behaftet (siehe Kap.3.2.6). Der theoretische Wert für den XRD-Ordnungs-Parameter eines vollständig geordneten LMCO Kristalls ergibt sich mittels XRD-Simulation (siehe Kap. 3.2.2) zu $S_{\text {theo }}(p=1)=10^{-2}$. Im Vergleich dazu sind die gemessenen XRD-OrdnungsParameter, die zwischen $3.5 \cdot 10^{-4} \leq S \leq 11 \cdot 10^{-4}$ liegen (Abb.43), eine Größenordnung kleiner. Weiterhin ist die geschätzte Größe der Nahordnung erheblich größer als die relative Menge der geordneten Phase in den TEM Messungen (Abb.45).

Im Allgemeinen können zwei verschiedene Typen von Defekten in der B-Platz-Matrix vorliegen: Punktdefekte (PD) und Antiphasengrenzen (APG). Die PD resultieren in einer monotonen Abnahme von $S$ mit steigender Anzahl der PD. Aus diesem Grund kann, bei einer Defektdichte von nur $2-8 \%$, die Diskrepanz zwischen $S$ und $S_{\text {theo }}$ nur mit PD nicht erklärt werden. Im Gegensatz dazu, können APG zu einer großen Varianz von $S$ führen: o.B.d.A. wird der XRD-Ordnungs-Parameter eines vollständig geordneten LMCO Kristalls mit einem Volumen $V$, der von einer APG in zwei phasenverschobene B-Platz-geordnete, kristalline Domänen mit dem Volumen $V_{1}$ und $V_{2}$ getrennt ist, auf

$$
S=\left|V_{1}-V_{2}\right| / V * S_{\text {theo }}(p=1)
$$

reduziert. Somit wird $S$ für $V_{1} \sim V_{2}$ stark reduziert und erreicht mit $V_{1}=V_{2}$ bei bereits relativ kleinen Defektdichten einen Wert von null. Um sowohl die Auswirkung von PD als auch von APG auf den XRD-Ordnungs-Parameter zu bestimmen, wurden XRDSimulationen (siehe Kap. 3.2.2) an Perowskit-Kristallen mit $N^{3}$ Einheitszellen und einer Nahordnungs-Defektdichte von 5\% durchgeführt. Die teilgeordneten Kristallmodelle wurden erstellt, indem zunächst ein ungeordneter Kristall mit zufälliger Co/Mn-Verteilung generiert wurde. Dieser Kristall wurde mittels Nächsten-Nachbar-Austausch ${ }^{19}$ bis zu einer Nahordnung von $p=95 \%$ geordnet $^{20}$. Für jede Nahordnung $p$ gibt es eine minimale Gittergröße $N_{\text {min }}$, für die die Anzahl der Defekte $N_{D}^{3}=N_{\text {min }}^{3} \cdot(1-p)$ groß genug ist um eine APG zu bilden: $N_{\text {min }}^{3} \cdot(1-p)>N_{\text {min }}^{2} \Rightarrow N_{\text {min }}>(1-p)^{-1}$. In Abb.49 sind die Daten der XRD Simulation für

\footnotetext{
${ }^{19}$ Ein B-Platz-Ion $B_{0}$ wurde zufällig gewählt. Führt ein Austausch mit einem der sechs Nachbarn zu einer Erhöhung der lokalen Ordnung, findet der Austausch statt. Die lokale Ordnung für jedes B-Platz-Ion $L O(B)$ ist dabei definiert als eine Summe über die Art der Nächsten-Nachbar-Ionen (Co oder $M n): M n-M n$ und $C o-C o$ Paare werden mit $-1, M n-C o$ Paare werden mit +1 gewertet. Wird die Summe $L O\left(B_{0}\right)+L O\left(B_{1}\right)$ durch den Austausch erhöht, findet dieser statt. Dieser Algorithmus wird durchgeführt, bis die gewünschte Nahordnung von $p=\Sigma_{B}^{N^{3}} L O(B) / 6 N^{3}$ erreicht ist.

${ }^{20}$ Die Umkehrung dieser Vorgehensweise (Induzierung von Unordnung in einen geordneten Kristall) führt nicht zur Ausbildung von APGs.
} 
den XRD-Ordnungs-Parameter $S$ als Funktion der Gittergröße $N$ für eine Nahordnung von $p=95 \%$ gezeigt. Der simulierte Verlauf von $S(N)$ fällt im Bereich $N<32$ stetig mit steigender Defektanzahl, d.h. mit steigender Gittergröße $N$, ab, bleibt jedoch wesentlich größer als die experimentell beobachteten Werte für $S$ (grauer Bereich in Abb.49). Wie vor Kurzem diskutiert, ist diese Reduktion von $S$ zurückzuführen auf PD. Im Bereich $N>32$ werden die simulierten Werte von $S$ breit gestreut und stark reduziert. Hier tauchen die APG auf, die den Kristall in phasenverschobene Volumina teilt und $S(N)$ in die Größenordnung der experimentellen Werte verschiebt. Das Vorhandensein von APG in der (111)-Ebene in den XRD-Messungen bzw. in der Ebene senkrecht zur Elektronenstrahlrichtung in den HAADF TEM-Messungen scheint der Ursprung für die Abweichung zwischen den XRD/TEM-Daten und der abgeschätzten Nahordnung aus den magnetischen Eigenschaften zu sein. Auch das Auftreten der arbiträren Schwankung des XRD-Überstrukturreflexes, durch die thermische Auslagerung von LMCO Filmen mit einer großen Nahordnung, lassen sich mit der Existenz von APG verstehen. Aus dem Grund, dass das experimentell observierte Probenvolumen viel größer als das Simulierte ist, und $S$ von der absoluten Anzahl der Defekte in der BPlatz-Matrix abhängt, ist die Nahordnung in den Proben tendenziell größer als, die in der Simulation verwendeten $95 \%$.

Die, aus dem STEM-EDX Experiment gewonnenen, atomar-aufgelösten, elementspezifischen Karten zeigen eine ungeordnete Phase an der Substrat/Film Grenzfläche. Dies kann der chemischen Durchmischung von STO/LMCO zugeschrieben werden. Tatsächlich führt z.B. eine Sr-Dotierung in dem LMCO System zur Bildung einer ungeordneten Phase [129]. In der geordneten Phase, weit weg von dem Substrat, sind überdies schwache Signale von $M n$ auf den Co-Plätzen (und umgedreht) vorhanden. Dieses Ergebnis lässt sich, wie schon diskutiert, auch mit Hilfe von APG erklären. Das wichtigste Resultat der lokalen Untersuchung der elektronischen Struktur, ist jedoch, dass sowohl in Bereichen mit großer als auch geringerer Überstruktur-Intensität der $\left(\mathrm{Co}^{2+} / \mathrm{Mn}^{4+}\right)$-Zustand dominiert. Diese Erkenntnis in Verbindung mit den magnetischen Eigenschaften lässt die Schlussfolgerung zu, dass tatsächlich in der gesamten Probe eine Nahordnung existiert, auch in den Bereichen in denen man keine Fernordnung beobachten kann (blaue Bereiche in Abb.45). In diesem Zusammenhang lassen sich möglicherweise auch die globalen XAS-Messungen von Sawatzky et al. [133] erklären.

In dem APG-Bild lassen sich die Bereiche verschiedener Überstruktur-Intensität in Abb.45 als Domänen deuten, die bezüglich der Projektionsebene unterschiedlich durch APG beeinflusst werden. Dieser Zusammenhang ist noch mal auf der rechten Seite in Abb.50 verdeutlicht. Die pinken Bereiche in der Überstruktur-Intensität-Karte können als Bereiche gedeutet werden, die entweder keine APG aufweisen oder bei denen das, durch APG phasenverscho- 


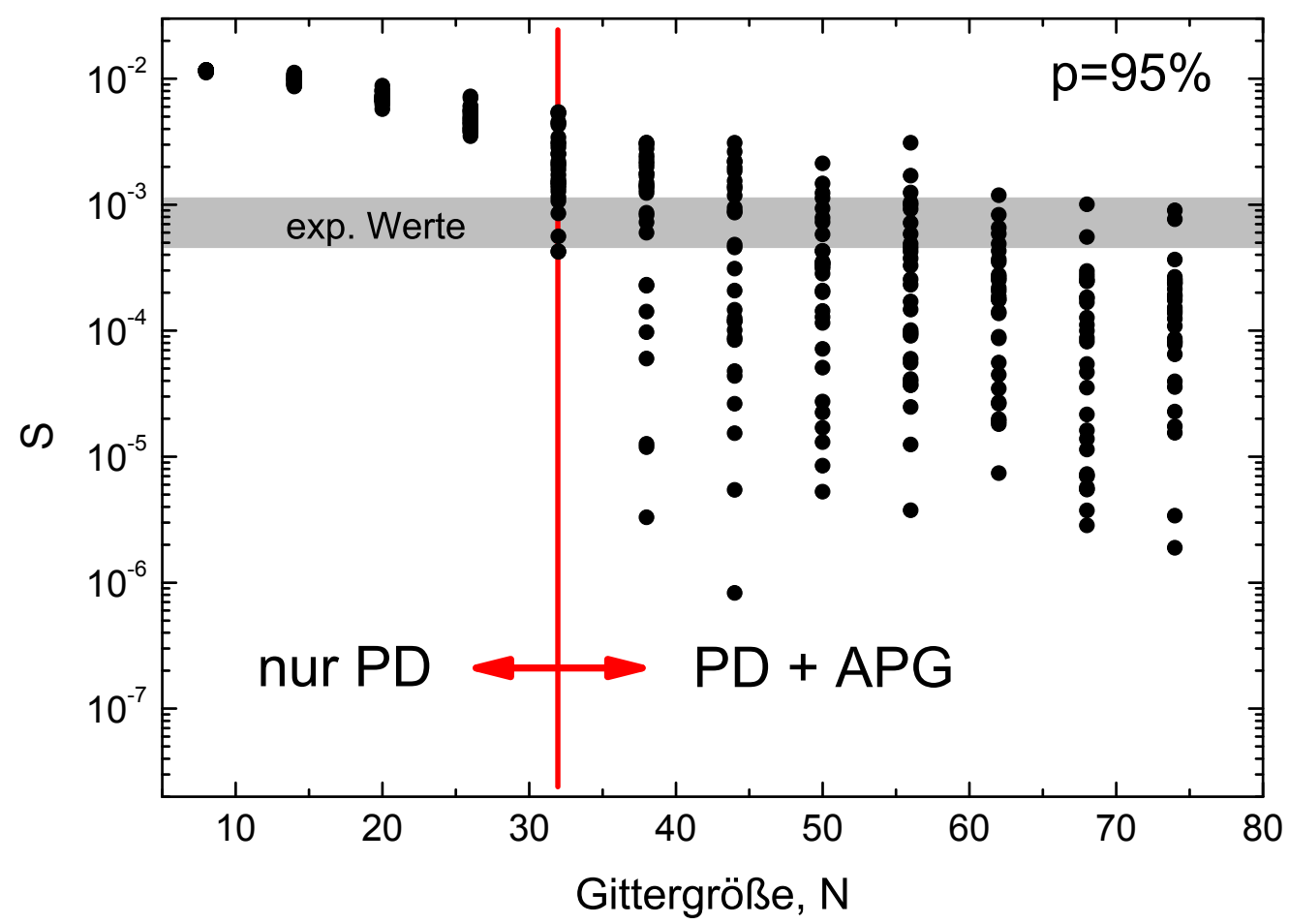

Abbildung 49: XRD Simulation für den XRD-Ordnungs-Parameter $S$ als Funktion der Gittergröße $N$ für eine Nahordnung von $p=95 \%$. Die kleine Varianz von $S$ für $N \leq 32$ zeigt die Abwesenheit von APG. Die experimentellen Werte von $S$ (grauer Bereich) werden nur für $N>32$ erreicht. In diesem Bereich ist die absolute Anzahl von B-Platz Defekten groß genug um, neben PD, auch APG zu bilden.

bene, Teilvolumen klein ist. In den blauen Bereichen ist dieses phasenverschobene Teilvolumen entsprechend Gleichung (10) größer. Mit Hilfe von Abb.50 lassen sich die APG-freien Domänen auf einen Durchmesser von 50 - 200nm abschätzen. Auch über die magnetischen Eigenschaften kann man eine Abschätzung für die APG-freie Domänengröße gewinnen. APGs manifestieren sich in der Reduktion der Kleinfeld-Magnetisierung aufgrund der AFM Kopplung zwischen dem phasenverschobenen FM Material, das durch die AFM-APG getrennt wird. In anderen ferro/ferrimagnetischen DP wird dies oft beobachtet, z.B. in $\mathrm{Sr}_{2} \mathrm{FeMoO}_{6}$ können APG zu einer starken Reduktion der Remanenz und Erhöhung des Koerzitivfeldes führen [122]. Bei einem bestimmten externen Feld $H$ überwiegt die Zeeman-Energie der FMDomäne $E_{Z e e m a n}$ der magnetischen Oberflächen-Energie der AFM-APG $E_{A F M}$. Bei diesem Feld wird die FM-Domäne ihre Magnetisierung parallel zum externen Feld ausrichten. wird eine kubische Domäne mit einer Kantenlänge $a$ angenommen, die aus kubischen Einheitszellen mit der Gitterkonstante $c$ aufgebaut ist, so ergibt sich für diese beiden Energien:

$$
E_{A F M}=6 a^{2} J_{A F M} / c^{2}=\mu_{0} H a^{3} M_{u . c .} / c^{3}=E_{Z e e m a n}
$$



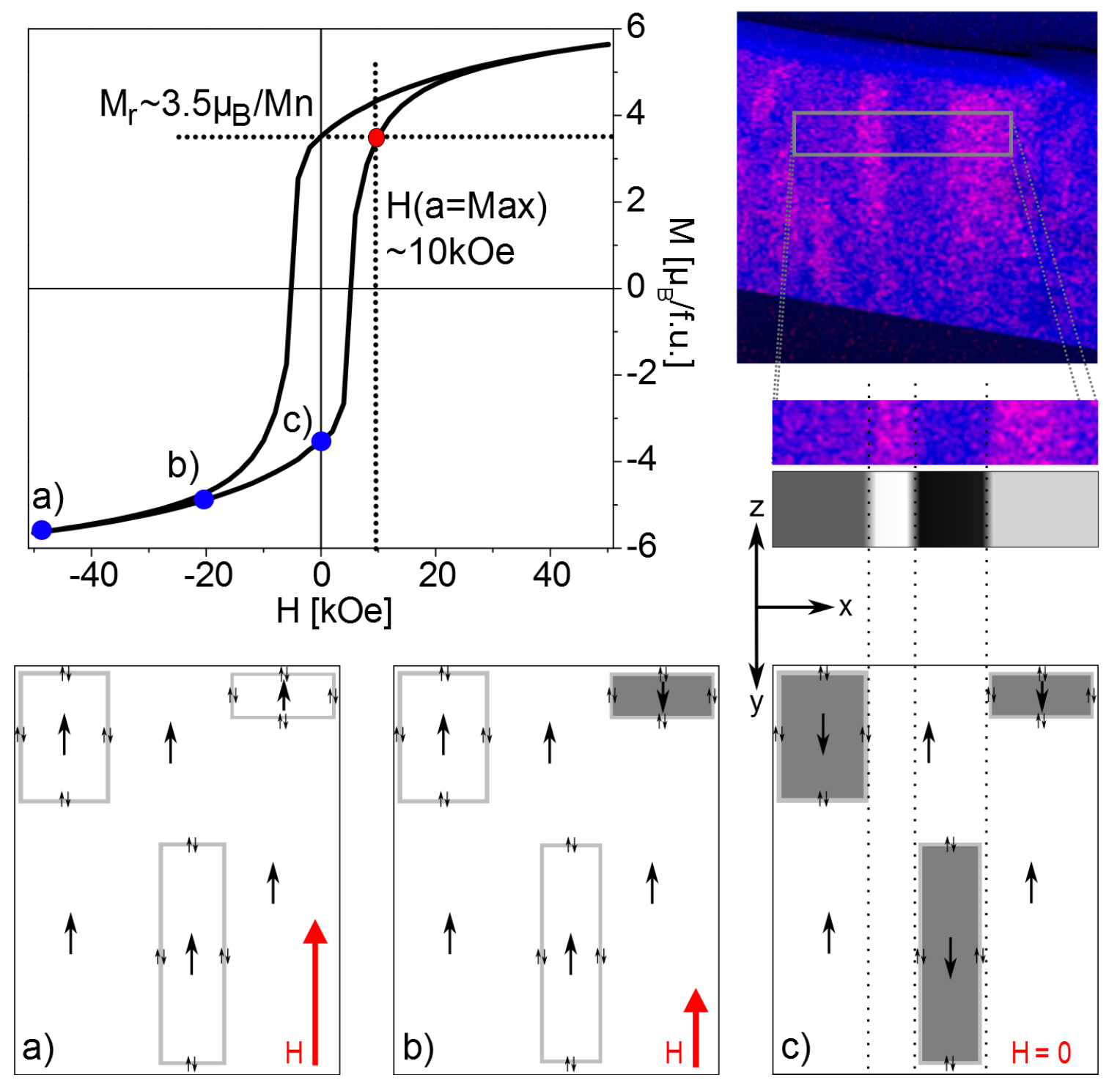

Abbildung 50: Model für die Magnetisierung der, durch APG phasenverschobene, Domänen: Die Überstruktur-Intensitäts Karte aus Abb.45 zeigt die Intensitätsunterschiede in der x-z-Ebene. Das kann als Anwesenheit von phasenverschobenen Domänen in der $x$-y-Ebene interpretiert werden. Diese Domänen haben einen Einfluss auf die Magnetisierung: a) Bei hohen Feldern ist die Magnetisierung fast aller Domänen parallel zum exterenen Feld ausgerichtet. b) Reduziert man das externe Feld richtet sich die Magnetisierung kleinerer Domänen antiparallel zum Feld aus. c) Bei Nullfeld ist die Magnetisierung aller phasenverschobenen Domänen antiparallel zum umgebenen FM Material ausgerichtet. Die blauen Punkte skizzieren die Zustände in der Hystereseschleife. Berücksichtigt man die Ähnlichkeit zwischen der Remanenz $(H=0)$ und dem Punkt bei Feldumkehrung mit gleicher Magnetisierung $(H>0)$ (markiert durch den roten Punkt), erhält man das externe Feld, was benötigt wird, damit sich die Magnetisierung der größten phasenverschobenen Domänen parallel zum externen Feld ausrichtet. Die TEM- und Magnetisierungs-Daten sind vom selben Film mit $\rho=1.07$. 
Dabei ist $J_{A F M}$ die AFM-Kopplungs-Energie pro Antiphasen-Bindung. Setzt man die Magnetisierung $M_{u . c .}=3 \mu_{B}$ für LMCO ein, erhält man für die Domänengröße:

$$
a=\frac{2 c J_{A F M}}{\mu_{0} H \mu_{B}}
$$

$J_{A F M}$ kann im Fall der $M n^{4+}-O-M n^{4+}$ AFM-Kopplung über die Néel-Temperatur $T_{N}=145 \mathrm{~K}$, von $\mathrm{Ca}_{0.8} \mathrm{Sr}_{0.2} \mathrm{MnO}_{3}$ abgeschätzt werden [111]. In diesem Perowskit ist der durchschnittliche A-Platz Ionenradius näherungsweise der von $L a^{3+}$ [103], somit ist der Toleranzfaktor vergleichbar. Es gibt jedoch kein Perowskit-Material, dass die Abschätzung der $\mathrm{Co}^{2+}-\mathrm{O}-\mathrm{Co}^{2+}$ AFM-Kopplung erlaubt. Somit konnte nur die $\mathrm{Mn}^{4+}-\mathrm{O}-\mathrm{Mn}^{4+} \mathrm{AFM}-$ Kopplung in der Abschätzung der Domänengröße berücksichtigt werden. Das externe Feld, welches benötigt wird um die größten/kleinsten vorhandenen Domänen parallel zum externen Feld auszurichten, kann mit Hilfe des vereinfachten Modells in Abb.50 abgeschätzt werden. In diesem Modell wird davon ausgegangen, dass es keine ferromagnetische Domänen ohne APG gibt, insbesondere, dass die phasenverschobenen Domänen magnetisch eindomänig sind. Dadurch, dass mit 50kOe nahezu die maximale theoretische Magnetisierung erreicht ist, kann man davon ausgehen, dass (nahezu) alle Domänen parallel ausgerichtet sind (siehe Abb.50 a). Wird das externe Feld zurückgefahren, würde sich zunächst, nach Gleichung (12), aufgrund der AFM-APG, die Magnetisierung der kleineren Domänen antiparallel zum äußeren Feld ausrichten (siehe Abb.50 b). Bei $H=0$ würde die Magnetisierung aller phasenverschobenen Domänen antiparallel zur Richtung der remanenten Magnetisierung stehen (siehe Abb.50 c). Aus dieser Konfiguration ergibt sich eine Remanenz, die deutlich kleiner ist als die Sättigungsmagnetisierung $M_{r}<M_{s}$. An dieser Stelle lässt sich in diesem Modell das Gesamtvolumen der phasenverschobenen Domänen in der Probe über die Remanenz bestimmen. In Abb.50 ist $M_{r}=3.5 \mu_{B} / f$.u., somit sind $\sim 20 \%$ des Gesamtvolumens phasenverschobene Domänen, bei einer theoretischen Sättigung von $6 \mu_{B} / f$.u.. In der Hysterese-Kurve wird unter Feldumkehr bei einem bestimmten externen (negativen) Feld (hier $H=10 \mathrm{kOe}$ ) die Magnetisierung in der Größe der Remanenz erreicht (in Abb.50 markiert durch den roten Punkt). Geht man davon aus, dass bis zu diesem Feld alle FM-Domänen, die nicht durch APG getrennt sind, nahezu eindomänig sind, liegt an diesem Punkt eine ähnliche Konfiguration wie in Abb.50 c) vor. Erhöht man das externe Feld weiter, würden an diesem Punkt sich die Magnetisierung der größten phasenverschobenen Domänen parallel zum externen Feld ausrichten. Somit kann das Feldintervall, in dem die Magnetisierung die phasenverschobenen Domänen parallel zum Feld ausrichtet, mit 10 - 50kOe angegeben werden. Damit sind die Domänen zwischen $a=35-170 \mathrm{~nm}$ groß. Obwohl für diese Abschätzung viele Annahmen und Vereinfachungen gemacht werden mussten, ist dieses Ergebnis aus den magnetischen Daten in guter Übereinstimmung mit den TEM Resultaten desselben LMCO Film. Teilt 
man das Gesamtvolumen der phasenverschobenen Domänen (20\% vom Gesamtvolumen) auf $a=35$ - 170nm große Domänen auf, kann man die absolute Anzahl der B-Platz-Defekte im Film abschätzen. Die Nahordnung, die man mit Hilfe dieses Modells abschätzen kann liegt bei $p \sim 98.7-99.7 \%$ in der in Abb.50 untersuchten Probe mit $\rho=1.07$. Dieser Wert ist etwas Größer als die Nahordnung, die mit Hilfe der Sättigungsmagnetisierung auf einen minimalen Wert von $p=92-98 \%$ bestimmt wurde. Zusätzlich wurden bei der Ermittlung der Nahordnung über das APG-Modell nur Defekte gezählt, die zu den APG gehören, d.h. keine PD und auch keine ungeordneten Volumina. Damit ist gezeigt, dass man in diesem APG-Modell die strukturellen, magnetischen und elektrischen Eigenschaften dieser LMCO Filme erklären kann.

Die Variation des $C o / M n$-Verhältnisses in den La-defizitären LMCO Filmen hat keinen Einfluss auf die magnetischen Eigenschaften. Da der XRD-Ordnungs-Parameter $S$ mit XRD global bestimmt wurde und, wie bereits diskutiert, stark von APG beeinflusst wird, ist er kein quantitatives Maß für die Nahordnung. Somit unterscheiden sich diese Proben nur in der Gitterkonstante und der Co/Mn-Stöchiometrie der La-defizitären Ausscheidungen. Möglicherweise wird ein Teil des Co-Überschusses in die LMCO-Matrix eingebaut, angedeutet durch die Änderung der Gitterkonstante mit dem Co/Mn-Verhältnis. Der Großteil muss jedoch, aufgrund der magnetischen Eigenschaften und der daraus resultierenden Nahordnung von $p>92 \%$, von den La-defizitären Ausscheidungen aufgenommen werden. Dies wird bestätigt von den TEM Messungen, die den Ausscheidungen das Co-reiche $\mathrm{Co}_{2-x} \mathrm{Mn}_{x} \mathrm{O}_{3}$ oder $\mathrm{Co}_{3-x} \mathrm{Mn}_{x} \mathrm{O}_{4}$ Material, in Übereinstimmung mit dem Fremdphasenreflex im XRD, zuordnen. Erstaunlich ist die große Beweglichkeit von La und Co. Beide haben sich in der kurzen Zeit der Präparation $(<30 \mathrm{~min})$ über mehrere 100 $\mathrm{nm}$ bewegt, wodurch sich ein Diffusionskoeffizient bei der Depositionstemperatur $T_{D e p}$ von $D\left(T_{D e p}\right)>$ $3 \cdot 10^{-15} \mathrm{~cm}^{2} / \mathrm{s}$ abschätzen lässt. Dies ist im Vergleich mit der Diffusion in nicht-defizitären Manganat/Manganat Übergittern mit $D\left(T_{D} e p\right)<5 \cdot 10^{-19} \mathrm{~cm}^{2} / \mathrm{s}$ (siehe Kap. 4) sehr hoch. Insbesondere ist bekannt, dass in dem LMCO ähnlichen $L a_{2} N i M n O_{6}$ System, die $L a-$ Leerstellen stabil sind und keine hohe Diffusivität aufweisen [118]. Der Einfluss von LaLeerstellen in LMCO wurde bisher in der Literatur nicht untersucht. Der LMCO Film ohne $L a$-Defizit konnte das Co/Mn-Missverhältnis nicht ausgleichen, es entstand ein teilgeordnetes LMCO Material. Die thermische Auslagerung führte zu ähnlichen Eigenschaften, wie in den $L a$-defizitären Filmen. Man kann daraus schließen, dass die Präparation mit einem leichten La-Defizit, die Einstellung der B-Platz-Ordnung in LMCO fördert und gleichzeitig andere stöchiometrische Fehler bindet. Prinzipiell lässt sich feststellen, dass mit MAD LMCO Filme mit einer hohen Nahordnung $p>92 \%$ präpariert werden können. 


\subsection{Präparation von ferrimagnetischen Doppelperowskit Filmen mit MAD}

Bereits 1961 wurde in einigen DP auf Re-Basis[144], später auch in $W$ - und $M o$ - basierten DP[145], eine ferrimagnetisches Ordnung bei relativ hohen Temperaturen (zum Teil über Raumtemperatur) entdeckt. Zusätzlich besitzen einige dieser ferrimagnetischen DP eine hohe Leitfähigkeit und zeigen ein metallisches Verhalten [146]. 1998 wurde das wissenschaftliche Interesse für diese Materialien, durch die Entdeckung des halbmetallischen Charakters von $\mathrm{Sr}_{2} \mathrm{FeMoO}_{6}$ [147], für spintronische Anwendungen, wie z.B. Tunnel-MagnetowiderstandsElemente, geweckt. Durch die hohen Ordnungstemperaturen von $T_{C}>400 \mathrm{~K}$ kommen sie somit möglicherweise auch für Anwendungen bei Raumtemperatur in Frage [11, 120].

\subsubsection{Elektronische Struktur von $\mathrm{Sr}_{2} \mathrm{FeMoO}_{6}$}

Der meist studierte Vertreter der ferrimagnetischen halbmetallischen DP ist das $\mathrm{Sr}_{2} \mathrm{FeMoO}_{6}$ (SFMO). Der Curie-Punkt von SFMO liegt bei $\sim 420 K[145,148]$ und der Widerstandsverlauf zeigt ein metallisches Verhalten [147, 149]. Wie auch schon in LCMO (Kap. 5.1) gibt es in SFMO eine große Abhängigkeit der magnetischen, aber auch der elektronischen Eigenschaften, von der Fe-Mo-Ordnung [150-152]. Auch die Valenz-Zustände $\operatorname{des}(\mathrm{Fe} / \mathrm{Mo}$ )-Systems werden in der Literatur strittig diskutiert. Die summierte Valenz von $F e$ und $M o$ muss +8 betragen, somit ist sowohl ein $\left(F e^{3+} / M o^{5+}\right)$-Zustand, wie auch ein $\left(\mathrm{Fe}^{2+} / \mathrm{Mo}^{6+}\right)$ für dieses System vorstellbar. Mößbauer-, Röntgenabsorptions (XAS) und Photoemissions-Spektroskopie Experimente zeigen, dass sich der wirkliche Valenz-Zustand eher zwischen diesen beiden postulierten Zuständen bewegt: $\left(F e^{2.8+} / M o^{5.2+}\right)[151,153-155]$. Dabei ist das Fe, aufgrund der starken Spaltung der spin-up und spin-down Zustände durch die große Austauschwechselwirkung [147], in einem high-spin-Zustand und hat somit ein Spin-Moment von $S=5 / 2 \mathrm{im} \mathrm{Fe}^{3+}$-Fall bis $S=4 / 2 \mathrm{im} \mathrm{Fe}^{2+}$-Fall. Das $M o$ besitzt im $\mathrm{Mo}^{5+}$ Fall nur noch ein $4 d$-Elektron, somit bewegt sich das Spin-Moment des $M o$ zwischen $S=1 / 2$ und $S=0$. Sowohl Neutronenstreuung als auch zirkularer magnetischer Röntgendichroismus (XMCD) an SFMO Bulk-Kristallen zeigen eine antiferromagnetische (AFM) Kopplung zwischen $F e$ und $M o$, und somit einen ferrimagnetischen Zustand mit einer theoretischen max. Sättingungsmagnetisierung von $M_{s}=4 \mu_{B} / f . u$. [156-158]. Prinzipiell lassen sich die GKARegeln des SE, durch das Auftreten von delokalisierten Elektronen (metallisches Verhalten) und von nicht-diskreten Valenz-Zuständen, nicht anwenden um diese AFM-Kopplung zu erklären. Zudem ließe sich die große Austauschwechselwirkung, die zu einer Curie-Temperatur von $T_{C}=420 K$ führt nicht mit dem SE-Model erklären. In Verknüpfung mit dem Resultat, 


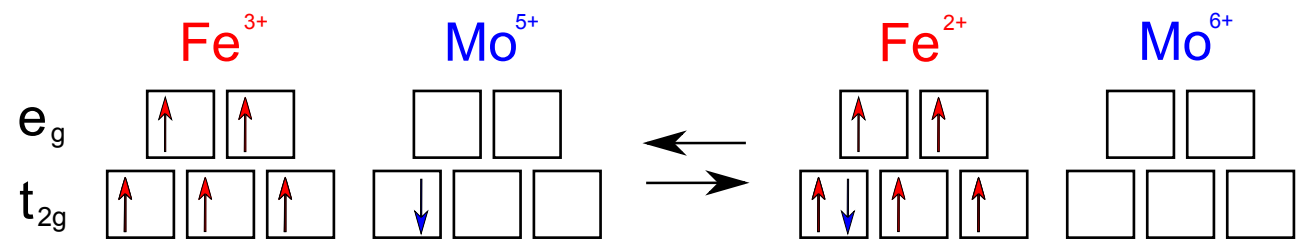

Abbildung 51: Modell ähnlich dem Zener-Doppelaustausch zur Erklärung der ferrimagnetischen Kopplung in SFMO: Das $t_{2 g}$-Elektron des Mo kann, wenn es einen Spin antiparallel zu den FeElektronen besitzt, über das Sauerstoff $2 p$-Orbital (hier nicht dargestellt) in das $t_{2 g}$-Orbital des Fe delokalisieren und damit die Energie im System minimieren.

dass das Elektron mit dem Minoritäts-Spin (das $4 d-t_{2 g}$-Elektron des $M o^{5+}$ ) in die $F e$ - und $M o-t_{2 g}$-Orbitale über das $2 p$-Orbital des Sauerstoffes delokalisiert (Halbmetall nach [147]), wurde ein Model ähnlich dem Zener-Doppelaustausch (siehe Kap. 2.2) vorgeschlagen um die magnetischen Eigenschaften des SFMO und ähnlicher DP zu verstehen [159]. Dieses Model ist in Abb.51 skizziert. Im Kontrast zum Zener-Doppelaustausch findet der Elektronenaustausch zwischen den $t_{2 g}$-Orbitalen statt und es stellt sich, aus dem Grund, dass sich ein Elektron mit Minoritäts-Spin delokalisiert, eine AFM-Kopplung ein. Anhand dieses Models erfährt man auch die Abhängigkeit der magnetischen und elektronischen Eigenschaften dieses Materials von der B-Platz-Ordnung: Das Elektron kann nur dann delokalisieren und zu einer Energieminimierung im System führen, wenn die magnetischen Momente der Fe-Ionen ferromagnetisch zueinander ausgerichtet sind. Dies geschieht nur über die AFM-Kopplung zum Mo. Damit ist eine Ordnung der Abfolge $F e-M o-F e-M o-.$. notwendig zur Realisierung dieser magnetischen und elektronischen Eigenschaften.

\subsubsection{Problematik in der Präparation von ferrimagnetischen DP Filmen}

Ein Blick auf die freie Enthalpie einfacher Metalloxidationen zeigt die große Problematik in der Synthese von ferrimagnetischen DP. In Abb.52 ist dies exemplarisch für SFMO dargestellt. Hier sind die temperaturabhängigen freien Enthalpien für die Oxidation von verschiedenen $F e$ - und Mo-Oxiden aufgetragen [160]. Die summierte Valenz von $F e$ und $M o$ ist durch den roten Pfeil dargestellt, diese reicht von +9 bis +2 . Die benötigte summierte Valenz zur Bildung von SFMO liegt, wie bereits festgestellt, bei +8 . Die Depositionstemperatur für Perowskite in der MAD liegt in einem Bereich zwischen $800-1000^{\circ} \mathrm{C}$, dies ist durch den grauen Bereich dargestellt. Wählt man eine Temperatur von $T_{D e p} \sim 930^{\circ} C$, liegt der Bereich, in dem diese summierte Valenz erreicht wird schätzungsweise bei dem in grün eingezeichneten Punkt. Die Positionierung des thermodynamischen Zustandes, kann neben der Temperatur, die für die Synthese von komplexen Oxiden beschränkt ist, durch 


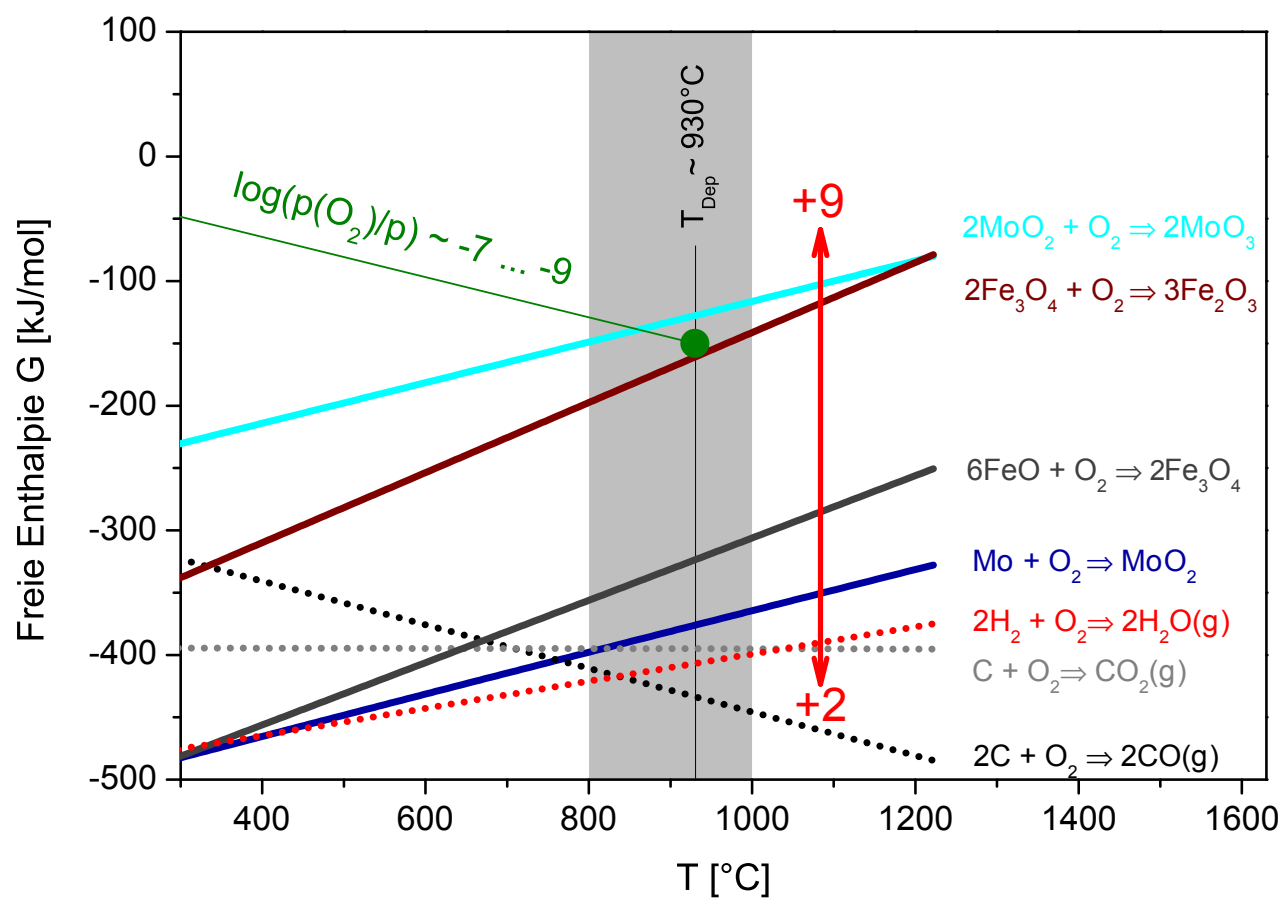

Abbildung 52: Die temperaturabhängige freie Enthalpien für die Oxidation von $C$ (gepunktete graue/schwarze Gerade), $\mathrm{H}_{2}$ (gepunktete rote Gerade) $\mathrm{FeO}$ (dunkelgraue Gerade), $\mathrm{Fe}_{3} \mathrm{O}_{4}$ (braune Gerade), Mo (dunkelblaue Gerade) und $\mathrm{MoO}_{2}$ (hellblaue Gerade) aus [160] sind aufgetragen. Die summierte Valenz von Fe und Mo ist durch den roten Pfeil dargestellt, diese reicht von +9 bis +2. Der thermodynamische Zustand, der durch den grünen Punkt markiert ist, kann mit der Einstellung der Temperatur und des Sauerstoffpartialdruckes eingeregelt werden. Der Sauerstoffdruck kann dabei über die Ellingham-Konstruktion (grüne Gerade) bestimmt werden [161].

den Sauerstoffpartialdruck gesteuert werden. In der in Abb.52 dargestellten Auftragung lässt sich der benötigte Sauerstoffpartialdruck zum erreichen eines beliebigen Punktes innerhalb des Diagramms über die Ellingham-Konstruktion bestimmen [161]. Dieser liegt für den grünen Punkt bei $p_{\mathrm{O}_{2}} / p \sim 10^{-9} \ldots 10^{-7}$. Mit Hilfe dieser thermodynamischen Daten, kann man abschätzen, dass bei einer Temperatur von $\sim 930^{\circ} \mathrm{C}$ ein Sauerstoffpartialdruck von $10^{-6}$ mbar bis $10^{-4}$ mbar innerhalb der umgebenden Atmosphäre mit einem Gesamtdruck von $p=1 b a r$ benötigt wird, damit man die $\mathrm{Sr}_{2} \mathrm{FeMoO}_{6}$ Phase erhält. In UltrahochvakuumMethoden zur Präparation von dünnen Schichten, wie PLD oder Sputtern, ist es möglich den benötigten Sauerstoffpartialdruck zu erzeugen, jedoch gibt es große Probleme bei dem Wachstum von phasenreinen, B-Platz-geordneten, stöchiometrischen, epitaktischen SFMO Schichten [162-164]. Dies ist ersichtlich über den Vergleich der Sättigungsmagnetisierung bei tiefen Temperaturen. Während Bulk-Kristalle mit $M_{s} \sim 4 \mu_{B} / f$.u. existieren [151, 165], liegt die Sättigungsmagnetisierung von SFMO Filmen zwischen $1-3 \mu_{B} /$ f.u.[162-164, 166]. 


\subsubsection{Erprobung der Low-Oxygen-MAD (LOMAD) Technologie}

Für eine chemische Methode, die unter der Umgebungsatmosphäre arbeitet, wie die MAD, ist die Präparation von SFMO Filmen unmöglich. Damit diese Oxide auch mit der MAD synthetisiert werden können, muss der Sauerstoffpartialdruck während der Deposition reguliert werden können. Aus diesem Grund wurde die MAD 1 Anlage (siehe Kap. 3.1.2) zu einer lowoxygen-MAD (LOMAD) modifiziert: Die Anlage wurde (soweit wie möglich) abgedichtet und mit einem piezoelektrischen Drucksensor und einem Floureszensverlust-Sauerstoff-Sensor der Firma SST Sensing ausgestattet. Das für die Aerosol-Erzeugung benötigte Druckgas, wurde durch Stickstoff mit einer Reinheit von 5.0 ersetzt. Der absolute atmosphärische Druck, der während der Deposition innerhalb der MAD Kammer weiterhin nahe dem Normaldruck liegen sollte, wird mit Hilfe einer ölfreien Kolbenpumpe der Firma Ilmvac und einem zweistufigen Drosselventil reguliert. Eine geringe Menge an Sauerstoff ist natürlich durch die Unreinheit des Stickstoffgases und durch die letzten undichten Stellen der Anlage während der Deposition vorhanden. Die Regulierung des Sauerstoffpartialdruckes sollte über den Relativdruck zwischen der MAD-Kammer und der umgebenen Atmosphäre erfolgen.

Diese neue Depositions-Methode sollte zunächst an ein-komponentigen Oxiden, die über ihre physikalischen Eigenschaften eine Aussage über ihren Sauerstoffanteil und damit über den Sauerstoffpartialdruck während der Deposition liefern können, erprobt werden. Für diese Aufgabe eignet sich das System der Eisenoxide: Das Hämatit $\alpha-\mathrm{Fe}_{2} \mathrm{O}_{3}$ ist ein verkanteter AFM mit $T_{N}=960 K$. Reduziert man den Sauerstoffanteil in dem Material, erhält man das thermodynamisch unstabile ferrimagnetische Maghemit $\gamma-\mathrm{Fe}_{2} \mathrm{O}_{3}$ mit einer CurieTemperatur von $\sim 960 K$. Weitere Sauerstoffreduktion bringt das thermodynamisch stabile Magnetit $\mathrm{Fe}_{3} \mathrm{O}_{4}$ hervor, ein Ferrimagnet mit $T_{C}=860 \mathrm{~K}$ [19]. Zusätzlich zu diesem Unterschied in den magnetischen Eigenschaften, sind die Raman-Spektren dieser Eisenoxide völlig verschieden [167]. Wie man auch in Abb.52 sehen kann, liefert das Eisenoxid-System eine Aussage über die Präparationsbedingungen für das SFMO System.

In Abb.53 a) und b) sind die Ergebnisse des ersten mit LOMAD erfolgreich präparierten Eisenoxidfilmes auf STO(110) dargestellt. Über die Röntgendiffraktion kann die Phase als $\mathrm{Fe}_{3} \mathrm{O}_{4}$ identifiziert werden [19] (schwarz in Abb.53 a). Es sind nur die (nn0)-Reflexe zu sehen. Zusätzlich wurde ein Eisenoxidfilm bei Standard MAD Bedingungen auf STO(110) präpariert (rot in Abb.53 a). Dieser Film offenbart nur einen Reflex bei $2 \theta=61.1^{\circ}$, dessen zugehörige Phase nicht identifiziert werden kann, insbesondere sind keine (nn0)-Reflexe einer $\mathrm{Fe}_{3} \mathrm{O}_{4}$ Phase vorhanden. Mit Hilfe MOKE-Messungen konnte gezeigt werden, dass der mit LOMAD präparierte $\mathrm{Fe}_{3} \mathrm{O}_{4}$ Film eine remanente Magnetisierung bei Raumtempera- 
5.2 Präparation von ferrimagnetischen Doppelperowskit Filmen mit MAD
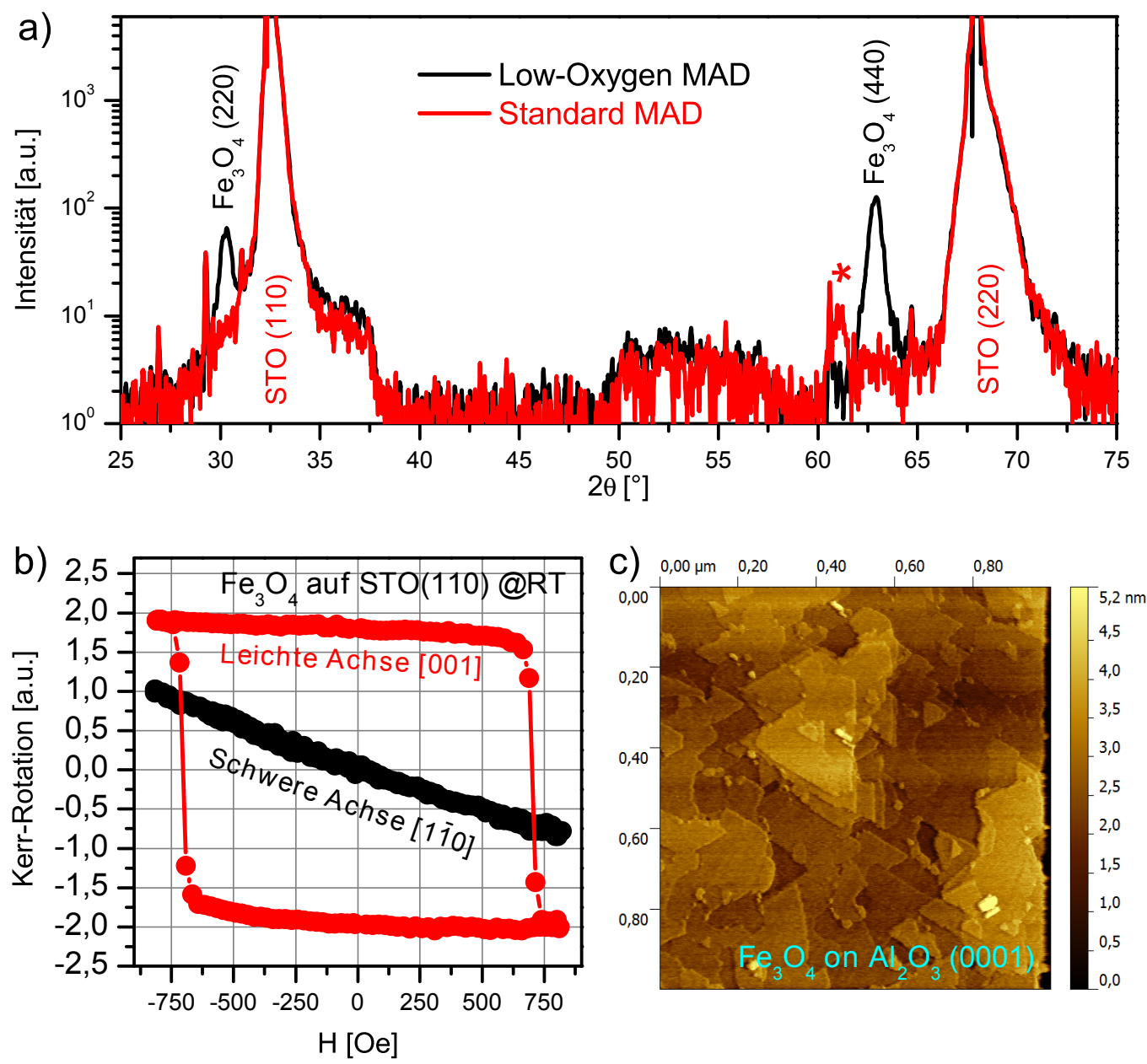

Abbildung 53: a) XRD Messung an zwei Eisenoxidfilmen auf STO(110) Substraten, hergestellt unter Standard-MAD Bedingungen (rot) und unter LOMAD-Bedingungen (schwarz). Dem LOMADFilm kann die $\mathrm{Fe}_{3} \mathrm{O}_{4}$-Phase zugeordnet werden [19]. Der Standard Eisenoxidfilm zeigt einen nicht zu identifizierenden Reflex, markiert mit *. b) MOKE Messung an dem LOMAD Fe ${ }_{3} \mathrm{O}_{4}$-Film auf STO(110) bei Raumtemperatur. Der Film zeigt eine remanente Magnetisierung. Zusätzlich ist die Magnetisierung des Filmes aufgrund der anisotropen Verspannung durch das STO(110) auch anisotrop (Magnetokristalline Anisotropie in $\mathrm{Fe}_{3} \mathrm{O}_{4}$ [168]). c) STM Aufnahme eines LOMAD $\mathrm{Fe}_{3} \mathrm{O}_{4}$ Filmes auf $\mathrm{Al}_{2} \mathrm{O}_{3}$ (0001). 
tur besitzt (siehe Abb.53 b). Der unter Standardbedingungen präparierte Film weist keine Magnetisierung in MOKE auf. Die Magnetisierung des $\mathrm{Fe}_{3} \mathrm{O}_{4}$ Filmes zeigt zusätzlich eine uniaxiale Anisotropie in der Filmebene: Die leichte Achse in der [001] Richtung (rot in Abb.53 b) zeigt ein Koerzitivfeld von 700Oe. Aufgrund des maximalen externen Feldes von $8000 e$ der Messapparatur, konnte in der [1ํㅣ] Richtung der schweren Achse (schwarz in Abb.53 b) nicht bis zur Sättigungsmagnetisierung gemessen werden. Somit kann die magnetokristalline Anisotropie beobachtet werden, die in einem anisotrop verspannten Film auf STO(110) $\mathrm{zu}$ erwarten ist [168]. Das bedeutet wiederum, dass der LOMAD- $\mathrm{Fe}_{3} \mathrm{O}_{4}$ Film epitaktisch verspannt auf dem STO(110) Substrat aufgewachsen ist.

Die Optimierung der Präparation von $\mathrm{Fe}_{3} \mathrm{O}_{4}$ mittels LOMAD wurde von Victor Pfahl im Zuge seiner Masterarbeit weiter verfolgt [169]. Innerhalb seiner Arbeit sollte vor allem überprüft werden, ob sich der Sauerstoff-Anteil des Films mit Hilfe des relativen Drucks innerhalb der MAD-Kammer, wie bereits postuliert, kontrollieren lässt. Für diese Experimente wurde die Präparationsbedingungen, mit Ausnahme des relativen Drucks der MAD Kammer $\Delta p$, konstant gelassen. Für die gesamte Proben-Serie war $\Delta p$ negativ $^{21}$, d.h. der Druck in der MAD war kleiner, als der Druck der umgebenen Atmosphäre. Somit kann der für die Oxidation benötigte Sauerstoff, über die Undichtigkeiten der Anlage zu den Reaktionskomponenten hinzukommen. Es konnte eine Proportionalität zwischen der SauerstoffleerstellenKonzentration und $\Delta p$ gezeigt werden [169]. Darüber hinaus existieren die Werte $\Delta p_{\min }$ und $\Delta p_{\max }$ : Eisenoxidfilme, die mit $\Delta p<\Delta p_{\min }=18$ mbar präpariert wurden zeigen eine Fremdphase, der Zementit $\mathrm{Fe}_{3} \mathrm{C}$ zugeordnet werden kann. Entsprechend findet man in Eisenoxidfilmen mit $\Delta p>\Delta p_{\max }=37$ mbar Hämatit oder Maghämit Fremdphasen. Insbesondere konnten die Präparationsbedingungen so gewählt werden, dass der Magnetit-Film einen Verwey-Übergang zeigt [169]. Das Auftreten dieses magnetischen Phasenüberganges hängt stark von der exakten Sauerstoffstöchiometrie in $\mathrm{Fe}_{3} \mathrm{O}_{4}$ ab [170]. In Abb.53 c) ist die Oberflächenmorphologie eines LOMAD- $\mathrm{Fe}_{3} \mathrm{O}_{4}$ Films auf einem $\mathrm{Al}_{2} \mathrm{O}_{3}$ (0001) Substrat, der mit optimierten Präparationsbedingungen gewachsen wurde, gezeigt. Es sind Stufen von der Höhe einer Gitterkonstanten zu sehen, welche die dreieckigen Terrassen der (111) Oberfläche voneinander trennen. Dieses STM Bild zeigt abschließend, dass die LOMAD Technik die Präparation von qualitativ hochwertigen Magnetit Filmen ermöglicht. Dabei können die Präparationsbedingungen, speziell der Sauerstoffpartialdruck über den relativen Druck innerhalb der MAD Kammer $\Delta p$ gesteuert werden.

Die Depositionstemperatur für SFMO Filme, die mit Hochvakuum-Techniken präpariert wurden, liegt mit 800 bis $950^{\circ} \mathrm{C}$ [162-164], wesentlich höher als die $\sim 700^{\circ} \mathrm{C}$, die für die LOMAD-

\footnotetext{
${ }^{21}$ Wenn im folgenden von $\Delta p$ gesprochen wird, ist dessen Betrag $|\Delta p|$ gemeint.
} 
5.2 Präparation von ferrimagnetischen Doppelperowskit Filmen mit MAD

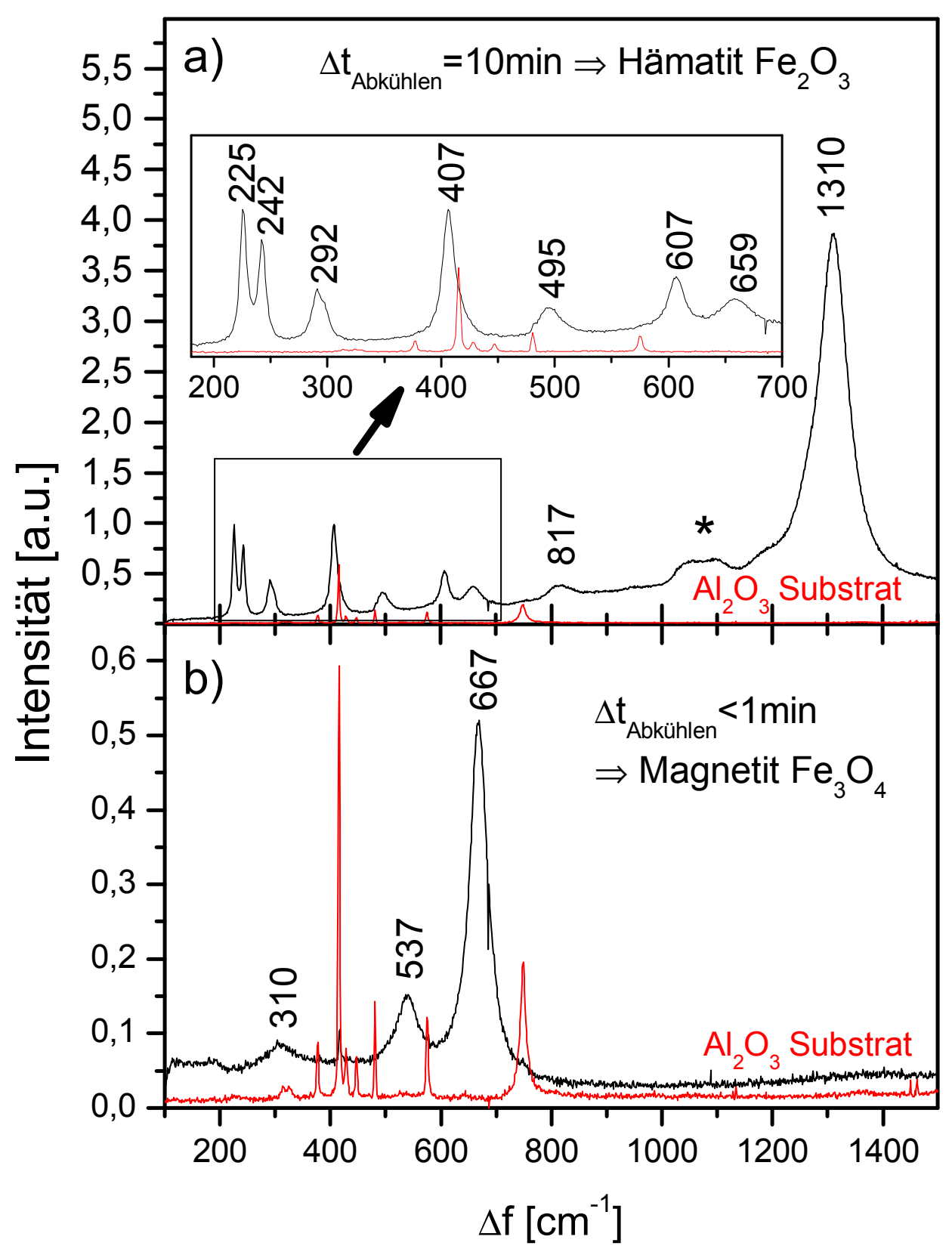

Abbildung 54: Ramanspektren von LOMAD Eisenoxidfilmen auf $\mathrm{Al}_{2} \mathrm{O}_{3}(0001)$ Substraten präpariert bei einer Depositionstemperatur von $\sim 930^{\circ} \mathrm{C}$ mit unterschiedlichen Abkühlraten: a) Bei Abkühlung innerhalb von 10min kann dem Spektrum Hämatit zugeordnet werden [167]. Der * Peak wird Verunreinigungen zugeschrieben [167]. b) Bei Abkühlung in weniger als 1min kann dem Spektrum Magnetit zugeordnet werden [167]. Kleine Beiträge des Substrates sind in beiden Filmspektren zu sehen. Das Spektrum des Substrats ist in rot hinzugefügt. 
$\mathrm{Fe}_{3} \mathrm{O}_{4}$ Filme verwendet wurde [169]. Aus diesem Grund wurde auch eine Präparation von $\mathrm{Fe}_{3} \mathrm{O}_{4}$ bei $\sim 930^{\circ} \mathrm{C}$ durchgeführt. Wie man anhand von Abb.52 sehen kann, muss bei einer Erhöhung der Depositionstemperatur der Sauerstoffpartialdruck entsprechend erhöht werden. Folglich muss $\Delta p$ für dieses Experiment gesenkt werden. Durch die Erhöhung der Depositionstemperatur ist auch die Gefahr der Einlagerung von Kohlenstoff aus den organischen Präkursoren in den entstehenden Film verringert. In Abb.52 sind zusätzlich die temperaturabhängigen freien Enthalpien für die Oxidation von Kohlenstoff präsentiert. Bei niedrigeren Temperaturen schneiden sich diese Geraden mit den Eisenoxid-Geraden. Aus diesem Grund konnte man die Anwesenheit einer $\mathrm{Fe}_{3} \mathrm{C}$-Phase für $\Delta p<\Delta p_{\min }$ in den MagnetitFilmen, die bei einer Depositionstemperatur von $\sim 700^{\circ} \mathrm{C}$ präpariert wurden, beobachten. Die Raman-Spektren von zwei bei $930^{\circ} \mathrm{C}$ deponierten Eisenoxidfilmen auf $\mathrm{Al}_{2} \mathrm{O}_{3}$ Substraten sind in Abb.54 dargestellt. Nähere Informationen zu den Parametern der Messung und der Messapparatur sind in der Masterarbeit von Victor Pfahl zu finden [169]. Die Präparation dieser beiden Filme unterscheidet sich nur in der Kühlrate nach der Deposition. Der Eisenoxidfilm in Abb.54 a) zeigt ein komplexes Spektrum, das im Vergleich mit Literaturdaten an Pulverproben [167] eindeutig dem Hämatit $\alpha-\mathrm{Fe}_{2} \mathrm{O}_{3}$ zugeordnet werden kann. Dieser Film wurde innerhalb von 10min von der Depositionstemperatur auf Raumtemperatur abgekühlt. Der Film dessen Ramanspektrum in Abb.54 b) dargestellt ist, wurde in weniger als 1 min auf Raumtemperatur abgekühlt ${ }^{22}$. Das Spektrum dieses Filmes kann eindeutig Magnetit $\mathrm{Fe}_{3} \mathrm{O}_{4}$ zugeordnet werden [167]. Aus diesem Ergebnis kann man folgern, dass nur während der Deposition der nötige Sauerstoffpartialdruck erreicht wird, der für die Synthese der $\mathrm{Fe}_{3} \mathrm{O}_{4}$ Phase benötigt wird. Bei der Abkühlung findet keine Oxidation der organischen Komponenten, die den Sauerstoff binden können, statt und der Sauerstoffpartialdruck steigt bei gleichen $\Delta p$ weiterhin an. Dadurch oxidiert der Film, der langsam abgekühlt wird, zu $\mathrm{Fe}_{2} \mathrm{O}_{3}$. Der Anstieg des Sauerstoffpartialdruckes wird von dem Sauerstoffsensor nicht registriert. Dies lässt sich nur damit erklären, dass der Sensor, der sich an der Kammerwand befindet, zu weit von dem Bereich, in dem während der Deposition die Pyrolyse und die Oxidation stattfindet, entfernt ist. Daraus lässt sich schließen, das der benötigte Sauerstoffpartialdruck nur in einem beschränkten Bereich, in dem die Oxidation der metallorganischen Komponenten der Lösung während der Deposition stattfindet, gegeben ist.

Die Funktionalität der LOMAD ist mit dem Eisenoxid-System gezeigt. Jedoch kann der Sauerstoffpartialdruck nicht direkt gemessen und somit auch nicht für zukünftige Depositionen als Vergleichsparameter heran gezogen werden. Es lässt sich jedoch ein Parameter definieren, der diese Forderung erfüllen kann: Wie bereits gezeigt, wird der Sauerstoffpartialdruck re-

\footnotetext{
${ }^{22} \mathrm{Im}$ Folgenden werden alle Filme, die in der LOMAD bei Depositionstemperaturen $T_{\text {Dep }}>700^{\circ} \mathrm{C}$ präpariert wurden, in dieser Weise abgekühlt.
} 
guliert, indem Sauerstoff durch die Druckdifferenz $\Delta p$ hinzugefügt und durch die Oxidation der metallorganischen Lösung gebunden wird. Geht man von der vollständigen Oxidation der organischen Komponenten aus, was sowohl für SFMO als auch für Eisenoxid für die optimalen Präparationsbedingungen gegeben ist (siehe Abb.52 ${ }^{23}$ ), lässt sich die molare Menge des Sauerstoffes berechnen, der gebunden wird. Über die Injektionsrate der Präkursorlösung in die LOMAD kann die molare Menge an Sauerstoff berechnet werden, die pro Zeiteinheit durch die Oxidationsreaktion gebunden wird. Ein Beispiel dazu: Löst man $50 \mathrm{mg} F e(a c a c)_{3}$ Präkursor in $V_{t o t}=3 \mathrm{ml}$ DMF und deponiert $V_{\text {Dep }}=0.5 \mathrm{ml}$ dieser Lösung, wird während der Deposition unter einer vollständigen Oxidation eine $\mathrm{O}_{2}$-Stoffmenge von:

$$
n\left(O_{2}\right)=\left(f_{F e(a c a c)_{3}} \cdot \frac{m_{F e(a c a c)_{3}}}{M_{F e(a c a c)_{3}}}+f_{D M F} \cdot \frac{V_{t o t} \cdot \rho_{D M F}}{M_{D M F}}\right) \cdot \frac{V_{D e p}}{V_{t o t}}
$$

verbraucht. Dabei ist $M_{x}$ die molare Masse, $\rho_{x}$ die Dichte und $m_{x}$ die Masse des Stoffes $x$. Der Faktor $f_{x}$ ist die Anzahl der Sauerstoffmoleküle, die bei der vollständigen Oxidation des Stoffes $x$ verbraucht werden. Für die Oxidation von DMF erhält man über:

$$
2 \mathrm{C}_{3} \mathrm{H}_{7} \mathrm{NO}+8.5 \mathrm{O}_{2} \rightarrow 6 \mathrm{CO}_{2}+7 \mathrm{H}_{2} \mathrm{O}+\mathrm{N}_{2}
$$

einen Faktor von $f_{D M F}=4.25$. Bei dieser Rechnung wird berücksichtigt, dass der Stickstoff im DMF nicht oxidiert wird, wie man anhand der thermodynamischen Daten erkennen kann [171]. Deponiert man die gegebene Lösung innerhalb von $60 \mathrm{~s}$, werden ca. $0.47 \mathrm{mmol}$ Sauerstoff pro Sekunde gebunden. Nimmt man außerdem eine laminare Strömung für die einströmende Sauerstoffmenge an, dann ist die Sauerstoffmenge pro Zeiteinheit proportional zur Druckdifferenz zwischen der MAD-Kammer und der umgebenen Atmosphäre ${ }^{24}$. Somit lässt sich aus den Präparationsparametern ein Quotient $\Omega$ bilden, der das Verhältnis zwischen den, durch Oxidation gebundenen, und den, durch Undichtigkeiten einfließenden, Sauerstoff definiert:

$$
\Omega=\frac{n\left(O_{2}\right)}{t_{D e p} \cdot \Delta p}
$$

wobei in der $t_{D e p}$ langen Deposition bei einer Druckdifferenz von $\Delta p$ die Stoffmenge des Sauerstoffs $n\left(\mathrm{O}_{2}\right)$ bei einer vollständigen Oxidation verbraucht wurde. Im Magnetit-System, für eine Depositionstemperatur von $\sim 700^{\circ} \mathrm{C}$ ergeben sich folgende Werte für $\Omega$ aus den Präparationsparametern [169]. Für das optimale Magnetit mit Verwey-Übergang ist $\Omega=$ $15.4 \mathrm{mmol} \mathrm{s}^{-1} \mathrm{bar}^{-1}$, für $\Omega \geq 17.9 \mathrm{mmol} \mathrm{s}^{-1}$ bar $^{-1}$ taucht die Zementit-Phase und für $\Omega \leq$

\footnotetext{
${ }^{23}$ Die freie Enthalpie für die Oxidation von $S r$ ist nicht in Abb.52 präsentiert, da diese weit unterhalb der gezeigten Werte liegt. Dies bedeutet auch, dass an jedem Punkt in Abb.52 das vollständig oxidierte $\mathrm{SrO}$ vorliegt.

${ }^{24}$ Nach Hagen-Poiseuille ist der Volumenstrom proportional zur Druckdifferenz $\dot{V} \sim \Delta p$.
} 
$14 \mathrm{mmol} \mathrm{s}^{-1} \mathrm{bar}^{-1}$ die Hämatit-Phase auf. Qualitativ eignet sich der Quotient $\Omega$ zur Abschätzung des Sauerstoffpartialdruckes. Ist er zu groß, ist der Sauerstoffpartialdruck zu klein und umgekehrt. Bei einer Depositionstemperatur von $\sim 930^{\circ} \mathrm{C}$ erhält man, wie die thermodynamischen Daten dies schon voraussagen (siehe Abb.52), auch bei $\Omega=4.5 \mathrm{mmol} \mathrm{s}^{-1} \mathrm{bar}^{-1}$, also bei einem höheren Sauerstoffpartialdruck eine reine Magnetit-Phase. Jedoch ist der Sauerstoffpartialdruck, der aus $\Omega$ resultiert, von dem Benötigten quantitativ weit entfernt. Dies geht aus den thermodynamischen Daten hervor (siehe Abb.52). Aus den $\Omega$-Werten des Magnetit-Systems ließe sich eine Abschätzung der maximalen Leckrate für die LOMAD Kammer gewinnen. Geht man davon aus, dass in den Proben in denen die Zementit-Phase auftaucht, nicht genügend Sauerstoff zur vollständigen Oxidation vorhanden ist, ist die Leckrate $L$ kleiner als der LOMAD-Parameter für diese Proben $L \leq 17.9 \mathrm{mmol} \mathrm{s}^{-1} \mathrm{bar}^{-1}$. Das bedeutet pro mbar Differenzdruck werden höchsten $17.9 \mu$ mol Sauerstoff pro Sekunde zusätzlich in die Anlage eingebracht. Mittels $L$ lässt sich wiederum der Sauerstoffüberschuß der Oxidation kalkulieren. Dieser liegt für die Depositionsbedingungen des stöchiometischen Magnetits bei $\sim 0.25 \mathrm{mmol} / \mathrm{s}$. Demgegenüber werden $\sim 8.9 \mathrm{mmol} / \mathrm{s}$ Stickstoffgas mit einer Sauerstoffverunreinigung von $0.04 \mu \mathrm{mol} / \mathrm{s}$ als Druckgas parallel in die Kammer injiziert. Im Bild des idealen Gases kann ein Sauerstoffpartialdruck von:

$$
\frac{p_{O_{2}}}{p_{\text {Ges }}} \sim \frac{n\left(\mathrm{O}_{2}\right)}{n\left(N_{2}\right)+n\left(\mathrm{O}_{2}\right)}=\frac{0.25 \mathrm{mmol}}{(8.9+0.25) \mathrm{mmol}} \sim 10^{-2}
$$

abgeschätzt werden ${ }^{25}$, der weit von den benötigten Sauerstoffpartialdrücken von $10^{-7}$ bis $10^{-17}$ [169] entfernt ist. Neben den Vereinfachungen, die in dieses Modell eingegangen sind (Thermodynamische Daten der einzelnen Komponenten, laminare Strömung, ideales Gas, vollständige Oxidation), gibt es möglicherweise auch konzeptionelle Probleme, die diese große Diskrepanz erklären können. Beispielsweise haben die Daten aus Abb.52 nur Gültigkeit für Systeme im thermodynamischen Gleichgewicht. Das muss für die LOMAD Deposition nicht der Fall sein. Man kann auch die Frage stellen ob die Metalloxide, die während der Pyrolyse entstehen gasförmig oder fest sind. Der Aggregatzustand hat einen großen Einfluss auf die freien Enthalpien und damit auf den Sauerstoffpartialdruck. Fragen dieser Art sind bisweilen ungeklärt, sowohl für LOMAD, als auch für Standard-MAD. Dessen ungeachtet eignet sich der LOMAD Parameter $\Omega$, wie bereits gezeigt, als qualitativer Vergleichsparameter zum Sauerstoffpartialdruck und als Orientierungsparameter für zukünftige Depositionen. Zu beachten ist, dass dieser Parameter jedoch von der Depositionstemperatur und von dem zu deponierenden Material abhängt.

\footnotetext{
${ }^{25}$ dabei ist $n(x)$ die Stoffmenge und $p_{x}$ der Druck des Stoffes $x$
} 


\subsubsection{Präparation von ferrimagnetischen $\mathrm{Sr}_{2} \mathrm{FeMoO}_{6}$ Filmen mittels LOMAD}

Wie bereits demonstriert eignet sich die LOMAD-Technik zur Deposition von ein-komponentigen Oxiden. Für die Präparation von komplexen Oxiden, müssen, wie auch bei der StandardMAD-Technik, zunächst die molaren Präkursoren-Verhältnisse bestimmt werden. $\mathrm{SrFeO}_{3-x}$ und $\mathrm{SrMoO}_{4}$ Filme wurden auf $\mathrm{STO}(001)$ Substraten $^{26}$ präpariert um das $\mathrm{Sr} / \mathrm{Fe}$ - sowie das $\mathrm{Sr} / \mathrm{Mo}$-Verhältnis zu bestimmen. In Abb.55 a) ist die XRD-Messung eines $\mathrm{SrFe-Oxid}$ Filmes dargestellt (in schwarz), der mit einem molaren Verhältnis von $S r / F e=1$ bei einer Depositionstemperatur von $T_{D e p} \sim 830^{\circ} \mathrm{C}$ und $\Omega=24.1 \mathrm{mmol} \mathrm{s}^{-1} \mathrm{bar}^{-1}$ präpariert wurde. Neben den zwei Fremdphasenreflexen (markiert mit *), sind die Reflexe einer RuddlesdenPopper-Phase des $\mathrm{SrFeO}_{3-x}$ zu erkennen, die texturiert auf dem Substrat aufgewachsen ist. Diese Phase kann mit $\mathrm{Sr}_{4} \mathrm{Fe}_{3} \mathrm{O}_{10-x}$ identifiziert werden [174]. Möglicherweise können die schwachen Fremdphasenreflexe einem kleinen, volumetrischen Anteil in dem Film, von einer oder mehreren anderen Ruddlesden-Popper-Phasen dieses Systems, zugeordnet werden. Vernachlässigt man diese Fremdphase vorerst, kann man schließen, dass das $\mathrm{Sr} / \mathrm{Fe}$-Verhältnis $\sim 1.34$ betragen muss. Dieser Wert wurde durch die Präparation eines weiteren Filmes bestätigt. Die XRD-Messung eines Films mit $T_{D e p} \sim 930^{\circ} \mathrm{C}, \Omega=19.6 \mathrm{mmol} \mathrm{s}^{-1} \mathrm{bar}^{-1}$ und $S r / F e=1.34$ ist zusätzlich in Abb.55 a) (in blau) aufgetragen. Aufgrund der pseudokubischen Gitterkonstante von $c=0.394 n m$ kann diesem Material die $\mathrm{SrFeO}_{2.5}$-Phase zugeordnet werden [172]. Neben den (00n) Reflexen sind keine weiteren Fremdphasen zu erkennen. Für die Präparation von $\mathrm{SrMoO}_{4}$ wurde zunächst auch ein $\mathrm{Sr} / \mathrm{Mo}$-Verhältnis von 1.34 gewählt. Die XRD-Messungen von zwei SrMo-Oxiden, die bei Standard-MAD-Bedingungen und einer Depositionstemperatur von $\sim 900^{\circ} \mathrm{C}$ hergestellt wurden, sind in Abb.55 b) dargestellt. Dabei wurde für diese zwei Filme unterschiedliche $M o$-Präkursoren verwendet. Die Messung des Films, welcher mit dem $\mathrm{MoO}_{2}(a c a c)_{2}$-Präkursor präpariert wurde (in schwarz), zeigt nur einen nicht zu identifizierenden Reflex, der mit * markiert ist. Es sind keine Reflexe zu erkennen, die einer $\mathrm{SrMoO}_{4}$-Phase zugeordnet werden können. Demgegenüber zeigt das Spektrum des Films, der mit dem $M o(C O)_{6}$ Präkursor präpariert wurde (in blau), die Anwesenheit von zwei verschieden orientierten SrMoO4-Phasen ohne das Auftreten einer Fremdphase [173]. Aus diesem Grund wurde für weitere Versuche an dem SFMO-System auf den $M o(C O)_{6}$ Präkursor zurück gegriffen und zunächst ein $\mathrm{Sr} /(\mathrm{Fe}+\mathrm{Mo})$-Verhältnis von 1.34 gewählt.

Im Folgenden wurden SFMO Filme sowohl auf STO(001) als auch auf STO(111) mit verschiedenen $\Omega$-Verhältnissen bei einer Depositionstemperatur von $\sim 930^{\circ} \mathrm{C}$ präpariert. Die

\footnotetext{
${ }^{26}$ Die Gitterfehlanpassung zwischen STO und $\mathrm{SrFeO}_{3-x}$ beträgt je nach Sauerstoffdefizit $-1.4 \%$ für $x=0$ und $0.7 \%$ für $x=0.5$ [172]. Die Gitterfehlanpassung zwischen STO und $\mathrm{SrMoO}_{4}$ beträgt $-2.4 \%$ [173].
} 
5 B-Platz Ordnung in ferri-/ferromagnetischen Doppelperowskiten
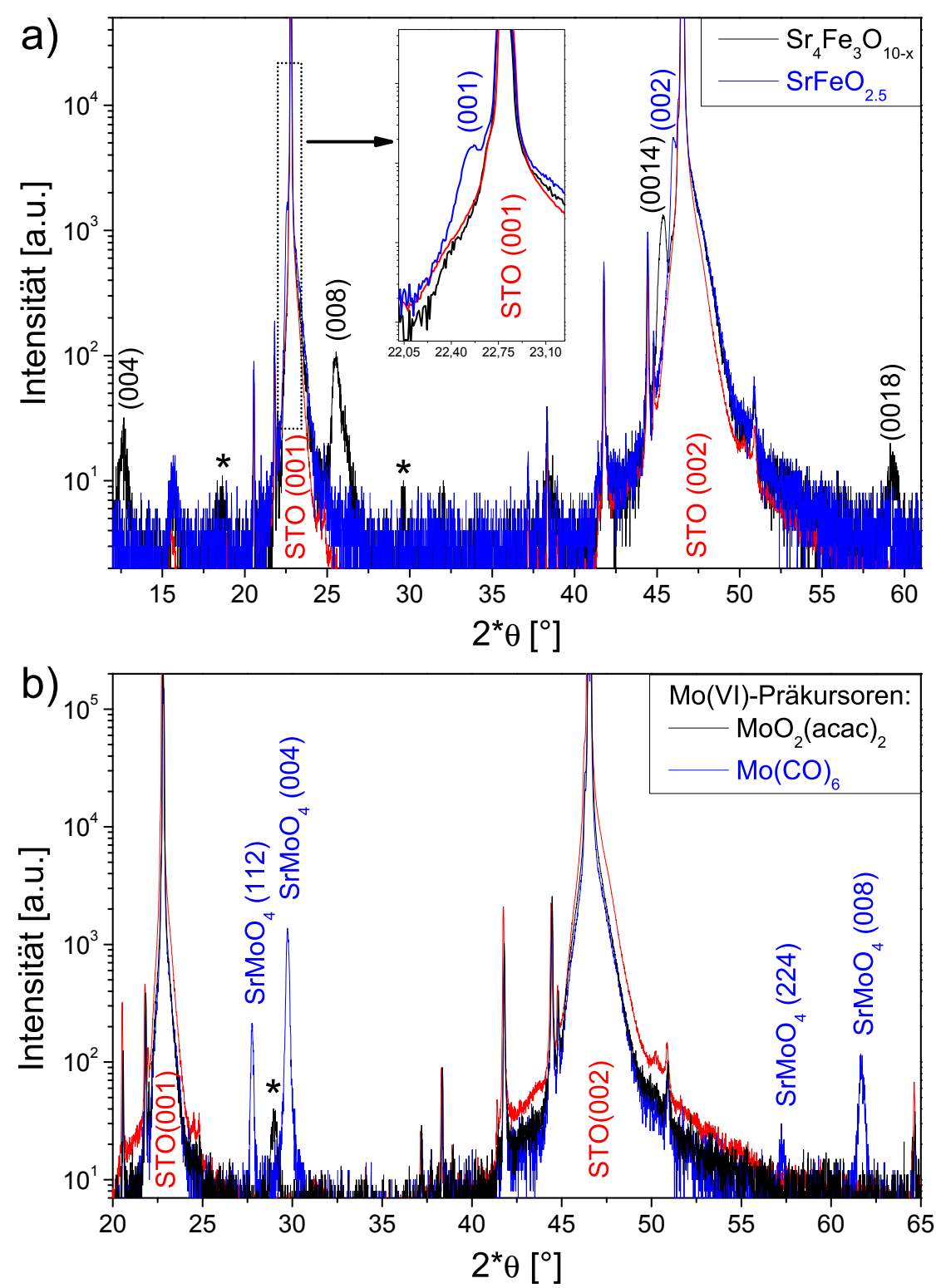

Abbildung 55: a)XRD-Messungen von $\mathrm{Sr}_{4} \mathrm{Fe}_{3} \mathrm{O}_{10-x}$ (schwarz) und $\mathrm{SrFeO}_{2.5}$ (blau) Filmen auf STO(001) Substrat, die mit der LOMAD Technik präpariert wurden. Die schwachen Fremdphasenreflexe (markiert mit *) können möglicherweise einer oder mehreren anderen Ruddlesden-PopperPhasen dieses Systems zugeordnet werden. b) XRD-Messungen von SrMo-Oxid-Filmen, die mit der Standard-MAD-Technik auf STO(001) Substraten präpariert wurden. Dabei kann die Phase des Films, der mit dem $\mathrm{Mo}(\mathrm{CO})_{6}$ Präkursor präpariert wurde (blau), als $\mathrm{SrMoO}_{4}$ identifiziert werden, während das Spektrum des Filmes, der mit $\mathrm{MoO}_{2}(\text { acac })_{2}$ präpariert wurde (schwarz), nur einen nicht identifizierbaren Reflex (markiert mit *) aufweist. In beiden Auftragungen ist zusätzlich das XRD-Spektrum des Substrates gezeigt (rot). 
XRD-Messungen für diese Filme sind in Abb.56 gezeigt. In der XRD-Messung des SFMO Films, der mit $\Omega=14.2 \mathrm{mmol} \mathrm{s}^{-1}$ bar $^{-1}$ präpariert wurde (schwarz in Abb.56 a), liegen neben dem SFMO(002) Reflex, mit Hilfe dessen sich die pseudokubische Gitterkonstante auf $c=0.396 \mathrm{~nm}$ bestimmen lässt, auch Fremdphasenreflexe vor. Diese Fremdphasen lassen sich zum einen als $\mathrm{SrMoO}_{4^{-}}$und zum anderen als SFMO(011)-Phase identifizieren. Das Auftreten der $\mathrm{SrMoO}_{4}$-Phase lässt den Schluss zu, dass der Sauerstoffpartialdruck für diese Präparation zu hoch war und der Film überoxidiert ist. Somit muss $\Omega>14.2 \mathrm{mmol} \mathrm{s}^{-1} \mathrm{bar}^{-1}$ sein, um einen einphasigen SFMO Film zu erhalten. Das Spektrum des $14.9 \mathrm{mmol} \mathrm{s}^{-1} \mathrm{bar}^{-1}$ SFMO Films (blau in Abb.56 a) zeigt neben den Substrat-Reflexen keine weiteren Reflexe. Jedoch zeigt die XRD-Messung des SFMO Filmes, der bei den gleichen Bedingungen auf einem $\operatorname{STO(111)~Substrat~gewachsen~wurde~(blau~in~Abb.56~b),~einen~schwachen~}$ Überstruktur (1/2 1/2 1/2)-Reflex bei $2 \cdot \theta=19.5^{\circ}$. Es ist kein weiterer Reflex dieser Orientierung erkennbar. Aus dem Überstrukturreflex erhält man mit $c=0.394 n m$ eine pseudokubische Gitterkonstante, die kleiner ist als die pseudokubische Gitterkonstante des überoxidierten SFMO. Aufgrund der hochintensiven Substratreflexe, sind die (nnn) und (00n)-Reflexe der SFMO-Phase umso schwieriger in der XRD-Messung zu erkennen, je kleiner die Gitterkonstante wird. Sowohl Sauerstoffleerstellen, als auch B-Platz Unordnung werden als Ursache für diese Gitterkonstanten-Änderung angesehen[166]. Die Intensität des Überstrukturreflexes eines weiteren SFMO Films, der mit $\Omega=16.3 \mathrm{mmol} \mathrm{s}^{-1} \mathrm{bar}^{-1}$ auf STO(111) präpariert wurde (schwarz in Abb.56 b), ist wesentlich kleiner als die Intensität des Überstrukturreflex des $14.9 \mathrm{mmol} \mathrm{s}^{-1} \mathrm{bar}^{-1} \mathrm{SFMO}$ Films und ist nur noch schwer von dem Rauschlevel der Messung zu unterscheiden. Auch in diesem Film ist kein weiterer Reflex der (nnn)-Orientierung erkennbar. Möglicherweise ist die pseudokubische Gitterkonstante in den Filmen mit $\Omega \geq 14.9 \mathrm{mmol} \mathrm{s}^{-1} \mathrm{bar}^{-1}$ zu klein um die Film-Reflexe von den Substrat-Reflexen zu unterscheiden. Es war außerdem möglich die Schichtdicke der $14.9 \mathrm{mmol} \mathrm{s}^{-1} \mathrm{bar}^{-1} \mathrm{SFMO}$ Filme zu bestimmen. Die Qualität dieser Filme war ausreichend gut, dass die Schichtdicke mittels XRR auf $\sim 93 \mathrm{~nm}$ bestimmt werden konnte. Es war nicht möglich die Schichtdicke für die 14.2 und $16.3 \mathrm{mmol} \mathrm{s}^{-1}$ bar $^{-1}$ SFMO Filme zu bestimmen.

In Abb.57 a) ist die temperaturabhängige Magnetisierung (Abkühlkurve) der SFMO Filme bei einem externen Feld von $1 k O e$ dargestellt. Alle Proben zeigen ein großes magnetisches Moment parallel zum externen Feld. Die Curie-Temperatur des $14.9 \mathrm{mmol} \mathrm{s}^{-1} \mathrm{bar}^{-1} \mathrm{SFMO}$ Films auf STO(111) liegt mit $T_{C} \sim 400 \mathrm{~K}$ nahe dem Literaturwert (blaue Kreise in Abb.57 a). Nach einer ca. 10 minütigen, thermischen Auslagerung bei $\sim 200^{\circ} \mathrm{C}$ an Luft zeigt der Film eine $\sim 20 K$ niedrigere Curie-Temperatur. Auch die Gesamtmagnetisierung hat sich vermindert (offene blaue Kreise in Abb.57 a). Offensichtlich ist der Film während der thermischen Auslagerung partiell überoxidiert, was zu einer Verminderung des magnetischen Moments führt. 
5 B-Platz Ordnung in ferri-/ferromagnetischen Doppelperowskiten
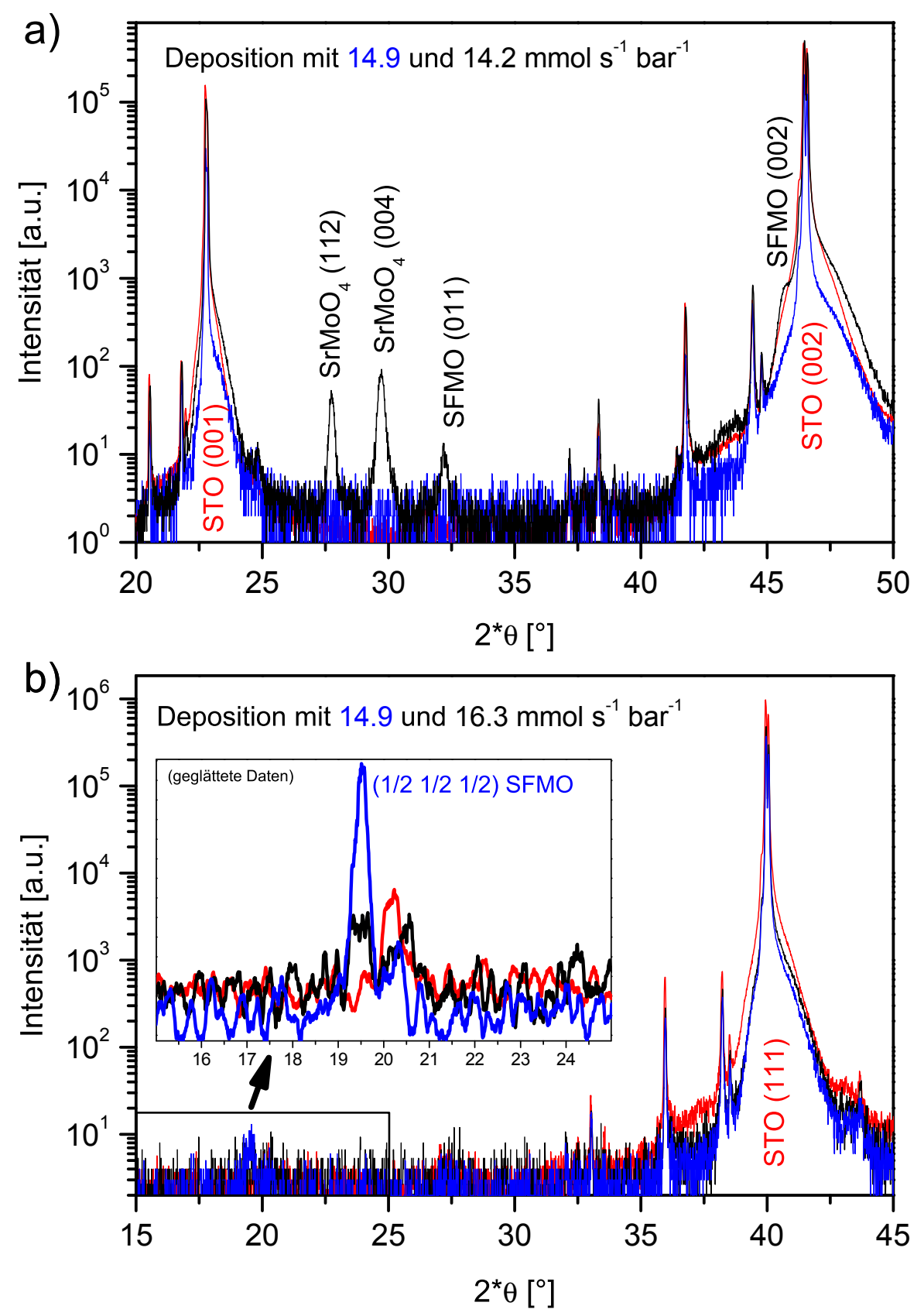

Abbildung 56: a) XRD-Messungen von SFMO Filmen die mit $\Omega=14.9$ (blau) und $14.2 \mathrm{mmol} \mathrm{s}^{-1} \mathrm{bar}^{-1}$ (schwarz) auf STO(001) gewachsen wurden. Der $14.2 \mathrm{mmol} \mathrm{s}^{-1} \mathrm{bar}^{-1} \mathrm{Film}$ zeigt die Anwesenheit einer zweiten SFMO-Orientierung und einer SrMoO4-Phase. b) XRDMessungen von SFMO Filmen die mit $\Omega=14.9$ (blau) und $16.3 \mathrm{mmol} \mathrm{s}^{-1}$ bar $^{-1}$ (schwarz) auf STO(111) gewachsen wurden. Das eingebettete Diagramm zeigt vergrößert die geglätteten Daten zwischen $15^{\circ}<2 * \theta<25^{\circ}$. Für den $14.2 \mathrm{mmol} \mathrm{s}^{-1}$ bar $^{-1}$ Film ist der (1/2 1/2 1/2)-Reflex bei $2 \cdot \theta=19.5^{\circ}$ klar vorhanden. In beiden Auftragungen ist zusätzlich die XRD-Messung des jeweiligen Substrates gezeigt (rot). 
Zusätzlich ist die temperaturabhängige Magnetisierung der 14.2 und $16.3 \mathrm{mmol} \mathrm{s}^{-1} \mathrm{bar}^{-1}$ Filme aufgetragen. Diese zeigen im Vergleich zum $14.9 \mathrm{mmol} \mathrm{s}^{-1} \mathrm{bar}^{-1}$ Film eine verminderte Curie-Temperatur von $\sim 370 K$. Auch das magnetische Moment ist kleiner und für den $14.2 \mathrm{mmol} \mathrm{s}^{-1} \mathrm{bar}^{-1}$ Film sogar fast nicht mehr vorhanden. Aufgrund der Tatsache, dass sowohl das deponierte Volumen als auch die Molarität der Präkursorlösung für alle hier präsentierten SFMO Filme gleich war, kann angenommen werden, dass die Stoffmenge von $M o$ und $F e$ in allen SFMO Filmen ähnlich ist. Daraus folgt, dass der quantitative Verlauf des magnetischen Momentes, dem quantitativen Verlauf der Magnetisierung dieser Proben entspricht. Betrachtet man die Größe der Curie-Temperatur und die der Magnetisierung als Maß für die Qualität des Films, ist der $14.9 \mathrm{mmol} \mathrm{s}^{-1} \mathrm{bar}^{-1}$ SFMO Film der hochwertigste in dieser Probenreihe. Die $M(H)$ Hysterese-Kurve des $14.9 \mathrm{mmol} \mathrm{s}^{-1} \mathrm{bar}^{-1}$ SFMO Films auf STO(111) bei $5 K$ nach dem Feldeinkühlen mit einem externen Feld von $1 k O e$ ist in Abb.57 b) aufgetragen. Es ist ein hysteretisches Verhalten, dass auf Ferri- oder Ferromagnetismus in diesem Film schließen lässt, mit einer Remanenz von $M_{r}=0.35 \mu_{B} / f$.u. und einer Sättigungsmagnetisierung von $M_{s}=0.9 \mu_{B} / f$.u. zu erkennen. Das eingebettete Diagramm in Abb.57 b) zeigt eine genauere Detailansicht der Nulldurchgänge der Hysterese-Kurve. Es ist erkennbar, dass die Kurve nicht punktsymmetrisch und nach links verschoben ist. Es ist ein "exchange bias" (EB) Effekt [175] in dieser Probe zu beobachten. Das exchange field $H_{E B}=H_{C 1} / 2-H_{C 2} / 2$ hat eine Größe von 38Oe. EB in SFMO wurde bereits in Nanopartikeln beobachtet [176]. In diesem Fall war der EB Effekt auf die Wechselwirkung zwischen den ferrimagnetischen SFMO Partikeln und deren Oberfläche, die ein Spin-Glass-Verhalten zeigt, zurückzuführen. Denkbar ist auch, dass das EB aus einer Wechselwirkung zwischen dem ferrimagnetischen SFMO und antiferromagnetischen ungeordneten SFMO zurückzuführen ist. Die genaue Bestimmung der Ursache für den EB wurde im Rahmen dieser Arbeit nicht durchgeführt, deshalb wird darauf auch nicht weiter eingegangen. Es kann festgestellt werden, dass sowohl das Auftreten von EB, als auch die niedrige Sättigungsmagnetisierung klar darauf hindeuten, dass der Film nicht vollständig geordnet ist. Benutzt man die Definition des Nahordnung-Parameters $p$ und die Erkenntnisse über die Wechselwirkung zwischen der B-Platz Ordnung und den magnetischen Eigenschaften aus Kap. 5.1.4 und die theoretisch maximale Sättigungsmagnetisierung von $4 \mu_{B} / f$.u., erhält man $p \sim 23 \%$. Dass heißt ca. $23 \%$ des Volumens des Films ist vollständig geordnetes ferrimagnetisches $\mathrm{Sr}_{2} \mathrm{FeMoO}_{6}$. Damit ist LOMAD die erste chemische Depositions-Methode mit der dieses System als dünner Film präpariert werden konnte.

Innerhalb dieser Arbeit ist es nicht gelungen einen vollständig geordneten SFMO mit der LOMAD zu präparieren. Es wurde jedoch gezeigt, dass dies möglich ist. Offensichtlich liegt der LOMAD-Parameter $\Omega$ für die Präparation eines nahezu vollständig geordneten SFMO zwi- 

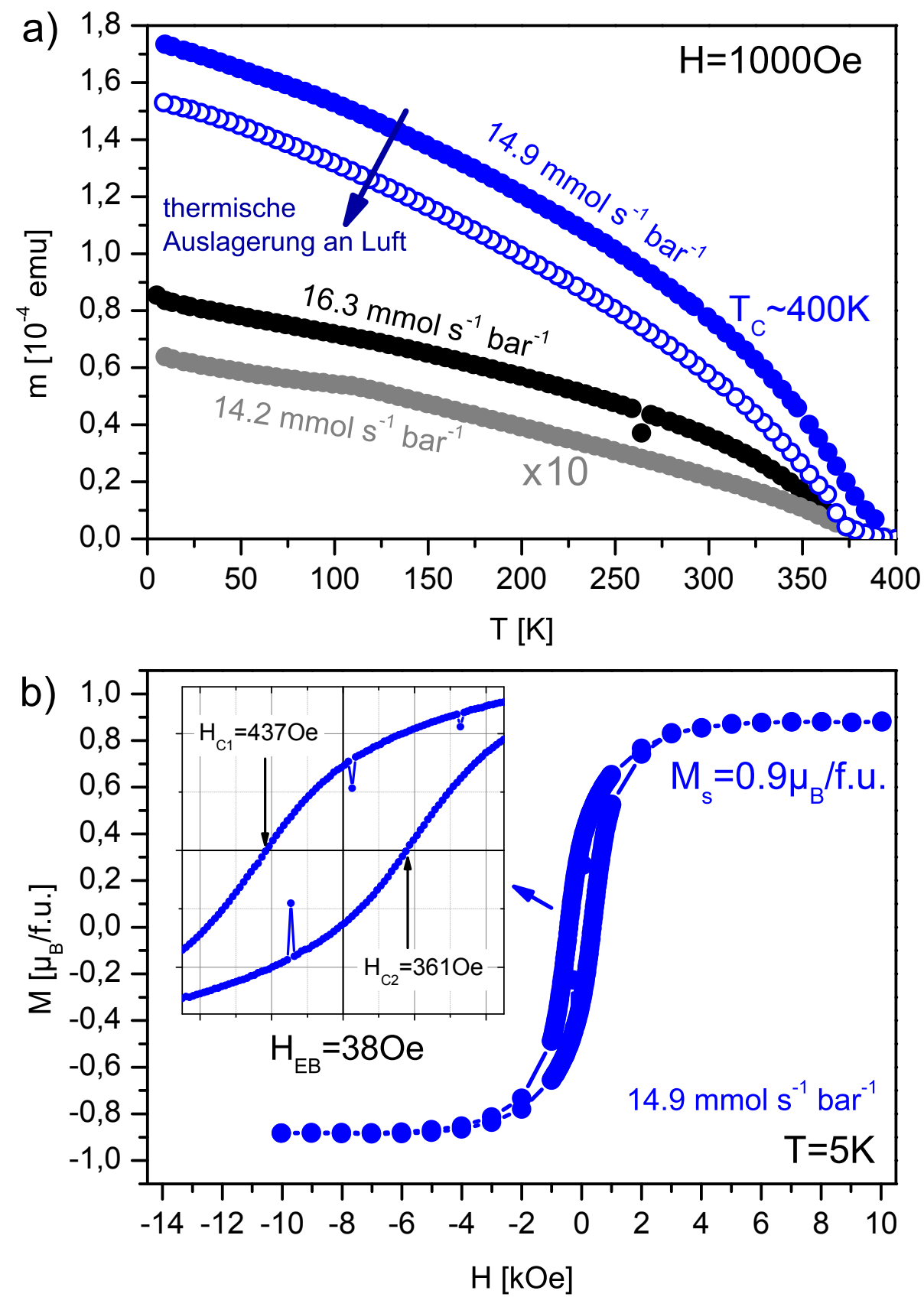

Abbildung 57: a) Das magnetische Moment $m$ in Abhängigkeit von der Temperatur $T$ (Einkühlkurven) bei einem externen Feld von $1 k O e$ für SFMO Filme, die mit $\Omega=14.2$ (grau, zehnfach vergrößert), 14.9 (blau) und $16.32 \mathrm{mmol} \mathrm{s}^{-1}$ bar $^{-1}$ (schwarz) präpariert wurden. Das magnetische Moment des $14.9 \mathrm{mmol} \mathrm{s}^{-1}$ bar $^{-1}$ Films nach der thermischen Auslagerung bei $\sim 200^{\circ} \mathrm{C}$ ist mit offenen blauen Kreisen dargestellt. b) Die $M(H)$ Hysterese-Kurve des $14.9 \mathrm{mmol} \mathrm{s}^{-1} \mathrm{bar}^{-1}$ Films bei $5 K$ nach dem Einkühlen mit $1 k O e$. Das eingebettete Diagramm zeigt eine Vergrößerung der Auftragung. Die Sättigungsmagnetisierung beträgt $0.9 \mu_{B} / f . u$., das exchange-field des exchangebias Effektes beträgt 38Oe. 
schen $14.2<\Omega_{S F M O}<16.3 \mathrm{mmol} \mathrm{s}^{-1} \mathrm{bar}^{-1}$ : Der $14.2 \mathrm{mmol} \mathrm{s}^{-1} \mathrm{bar}^{-1}$ Film ist überoxidiert (die Gesamtvalenz von $F e$ und $M o$ ist größer als +8 ), klar zu erkennen an dem Auftreten der $\mathrm{SrMoO}_{4}$-Phase. Die Magnetisierung des $16.3 \mathrm{mmol} \mathrm{s}^{-1} \mathrm{bar}^{-1}$ Films ist kleiner als die des $14.9 \mathrm{mmol} \mathrm{s}^{-1}$ bar $^{-1}$ Films. Damit ist dieser Film unteroxidiert (die Gesamtvalenz von $F e$ und $M o$ ist kleiner als +8 ). Der $14.9 \mathrm{mmol} \mathrm{s}^{-1} \mathrm{bar}^{-1}$ Film liegt innerhalb dieses Intervalls und zeigt eine hohe Curie-Temperatur, eine relativ hohe Sättigungsmagnetisierung und einen (1/2 1/2 1/2)-Überstrukturreflex. Bei zusätzlicher Sauerstoffbeladung durch thermisches Auslagern wird dieser weiter oxidiert und die magnetischen Eigenschaften entfernen sich weiter von denen, der optimalen SFMO Phase. Damit scheint $14.9 \mathrm{mmol} \mathrm{s}^{-1} \mathrm{bar}^{-1}$ nahe an dem optimalen Wert $\Omega_{S F M O}$ zu liegen, bei dem eine SFMO Phase entsteht deren Gesamtvalenz von $F e$ und $M o$ genau +8 beträgt.

\subsubsection{Präparation von ferrimagnetischen DP Filmen mittels LOMAD: Ausblick}

Das $\Omega$-Intervall, indem eine SFMO-Phase entstehen kann, ist im Vergleich mit Magnetit sehr eng. Daraus resultieren die großen Schwierigkeiten bei der Deposition dieses Materials mit der LOMAD. Um die SFMO Film-Qualität weiter zu verbessern, muss die LOMAD weiter überarbeitet werden, um eine genauere Definition von $\Omega$ zu ermöglichen. Von entscheidender Bedeutung ist dabei die Depositionsrate. Für alle LOMAD Filme wurde die Präkursorlösung von peristaltischen Pumpen transportiert und in die LOMAD injiziert. Diese Pumpen versprechen jedoch keine konstante Depositionsrate. Die Rate kann bei Depositionen mit den gleichen Bedingungen um mehr als 10\% abweichen (Reproduktionsfehler), was in dem SFMO $\Omega$-Intervall einen Fehler von etwa $1.5 \mathrm{mmol} \mathrm{s}^{-1} \mathrm{bar}^{-1}$ erzeugt. Dieser Fehler kann durch die Verwendung von Spritzendosierer, die die Präkursorlösung mit Hilfe von HochpräzisionsSchrittmotoren und -getriebe zuführen, minimiert werden. Ein anderer entscheidender Faktor ist die Sauerstoffzufuhr während der Deposition. In einer neuen völlig abgedichteten Anlage kann der Sauerstoff über einen Gasdosierer/mischer eingebracht werden. Diese Methode wäre kontrollierbarer und entfernt die Abhängigkeit des Depositionsprozesses von der Umgebungsatmosphäre und dem Differenzdruck in der MAD Kammer. Auch ein schnelles Abkühlen, um das weitere Oxidieren nach der Deposition zu verhindern, sollte nicht mehr nötig sein, da auf Wunsch die Sauerstoffzufuhr gestoppt werden kann. Mit diesen Modifikationen sollte die Präparation von qualitativ hochwertigen SFMO Filmen und auch von Heterostrukturen, aus Oxiden mit unterschiedlichen Anforderungen an den Sauerstoffpartialdruck, mit Hilfe der LOMAD-Technologie gelingen. 



\section{Zusammenfassung und Ausblick}

Die bereits präsentierten Ergebnisse der vier Themengebiete dieser Arbeit werden abschließend in diesem Kapitel in einen Kontext zusammengefasst.

Eine lagenweise A-Platz Kationen Ordnung bzw. Modulation in ferromagnetischen halbmetallischen Manganaten wurde innerhalb dieser Arbeit mit der Präparation von Multilagen-Systemen unterschiedlich dotierter Manganate realisiert. Die angestrebten ÜbergitterGeometrien konnten mit einem maximalen relativen Fehler von $<14 \%$ erreicht werden. Es konnte aufgezeigt werden, dass die, mit MAD präparierten, Manganat-Übergitter epitaktisch und mit einer hohen kristallinen Qualität auf Substraten mit guter (STO) sowie schlechter (MgO) Gitterpassung aufwachsen. Die strukturellen Untersuchungen offenbaren Unterschiede in der Schärfe der Manganat/Manganat- bzw. Manganat/Substrat-Grenzflächen und der Filmoberfläche, die von der Wahl der A-Platz Kationen im Manganat, des Substratmaterials und auch der Substratorientierung abhängen: Auf den MgO Substraten ist die Grenzfläche zum Manganat aufgrund des Mechanismus der "domain-matching-epitaxy" [92] für alle Manganate mit einer RMS-Rauigkeit von $\sigma \sim 1.0$ - 1.4nm aufgeraut, was auch zu relativ rauen Manganat/Manganat-Grenzflächen mit $\sigma \sim 0.4-0.7 n m$ auf diesen Substraten führt. Die Substrat/Manganat-Grenzfläche ist für SMO bzw. diverse Dotierungen von LSMO auf $\mathrm{STO}(001)$ mit $\sigma \sim 0.2-0.5 \mathrm{~nm}$ glatt, während sie für LMO mit $\sigma \sim 1.0-2.0 \mathrm{~nm}$ stark aufraut, was jedoch in diesem Fall nicht zu einer raueren Filmoberfläche führt. Die Erklärung für diese Beobachtung kann in der polaren Katastrophe [115] gefunden werden, in der sich das elektrische Potential, bei dem Wachstum von geladenen Perowskit-Halblagen auf einem neutralen Substrat, aufsummiert und durch die Bildung von Kristalldefekten abgebaut werden muss. Mit einem ähnlichen Ansatz lässt sich auch das Aufrauen des SMO Materials auf den STO(111) Substraten erklären. LMO bzw. LSMO wachsen hingegen glatt auf dieser Substratorientierung auf. Entsprechend der Beobachtungen in den einphasigen Dünnfilmen ist die Manganat/Manganat Grenzfläche für LMO/SMO Übergitter auf STO(001) mit $\sigma \sim 0.0$ - 0.25nm atomar scharf, während sie auf STO(111) wesentlich rauer ist. Es konnte nicht geklärt werden, warum die LSMO:0.5/LMO Übergitter relativ unscharfe Grenzflächen mit $\sigma \sim 0.3-0.6 \mathrm{~nm}$ in beiden untersuchten Substratorientierungen aufweisen.

Die lagenweise Ordnung oder Modulation der A-Platz Kationen induzierte in den ManganatSystemen eine Modulation der Gitterverspannung und der Elektronenbesetzung im $e_{g}$-Band der Manganionen, welche zu einer Änderung der magnetischen und elektronischen Eigenschaften führte: 
In den teilgeordneten $\left(\mathrm{La}_{0.5} \mathrm{Pr}_{0.5}\right)_{0.67} \mathrm{Ca}_{0.33} \mathrm{MnO}_{3}$ Schichten verursachte die Modulation der Gitterverspannung durch die lagenweise Ordnung von $L a$ und $\operatorname{Pr}$ eine Variation des relativen Volumenverhältnisses zwischen der FMM und AFM-COO-Phase im Bild der elektronischen Phasenseparation: Für hohe $n>4.5$ u.c. $=n_{\text {krit }}$ zeigen die elektronischen und magnetischen Eigenschaften der $\left((\mathrm{LCMO})_{n} /(\mathrm{PCMO})_{n}\right)_{m}$ Übergitter ein LCMO-artiges Verhalten, mit moderaten CMR-Werten, hohen Sättigungsmagnetisierungen $\sim 3 \mu_{B} / M n$ und CurieTemperaturen $T_{C}>200 \mathrm{~K}$. Unterschreitet man diese Grenze, nimmt das Teilvolumen der FMM-Phase stetig mit kleiner werdenden $n$ ab, während das Teilvolumen der AFM-COOPhase entsprechend ansteigt. Damit ist die Änderung der physikalischen Eigenschaften der Übergitter mit $n<n_{k r i t}$ analog zu LPCMO Filmen mit einem Pr-Anteil $y>0.5$. Insbesondere streben die magnetischen und elektronischen Eigenschaften der Übergitter mit kleiner werdenden $n$ auf die Eigenschaften der LPCMO $y=0.5$ Filme zu. Die Eigenschaften dieser Filme lassen vermuten, dass der AFM-COO-Anteil in LPCMO $y=0.5$ ähnlich hoch ist, wie in LPCMO $y=0.95$. Möglicherweise resultiert diese Unstetigkeit im Phasendiagramm von LPCMO auch aus einer Kationen-Ordnung der $L a, \operatorname{Pr}$ und $C a$ Ionen, die an dem Punkt $y=0.5$ mit gleich oft vertreten sind $\left(L a_{1 / 3} \operatorname{Pr}_{1 / 3} C a_{1 / 3} M n O_{3}\right)$. Diese Ordnung weicht jedoch geometrisch von der künstlichen, lagenweisen Ordnung der $\operatorname{La}$ und $\operatorname{Pr}$ Ionen ab, da diese Übergitter andere physikalische Eigenschaften zeigen. Interessanterweise ist $n_{\text {krit }}$ die Größenordnung der korrelierten Polaronen und die beobachteten physikalischen Eigenschaften lassen den Schluss zu, dass sich die COO Phase für $n<n_{k r i t}$ in das LCMO ausdehnt. Zukünftig sollte, mit der Hilfe einer TEM Investigation, die A-Platz Struktur von $\left(\mathrm{La}_{0.5} \mathrm{Pr}_{0.5}\right)_{0.67} \mathrm{Ca}_{0.33} \mathrm{MnO}_{3}$ und die COO-Phase in diesem Material untersucht werden, um diesen unsteten Punkt im LPCMO Phasendiagramm näher zu charakterisieren.

Über die lagenweise (Teil-)Ordnung von $L a$ und $S r$ in LSMO wird neben der Modulation der Gitterverspannung auch eine Modulation der Elektronenbesetzung des $e_{g}$-Bandes induziert. Generell fällt die Übergangstemperatur mit zunehmender Bilagen-Dicke sowohl in $\operatorname{dem}\left((\mathrm{LSMO}: 0.5)_{2 n} /(\mathrm{LMO})_{n}\right)_{m}$ - als auch in dem $\left((\mathrm{LMO})_{2 n} /(\mathrm{SMO})_{n}\right)_{m}$-System ab oder bleibt für kleine $n \leq 2$ in der (111) Orientierung auf nahezu einem Niveau. Dieses Verhalten konnte über den Vergleich mit dem $\mathrm{La}_{1-x} \mathrm{Sr}_{x} \mathrm{MnO}_{3}$ Phasendiagramm für dünne Filme auf STO(001) und STO(111) erklärt werden: Mit der Modulation befindet sich das LSMOMaterial im Phasendiagramm nicht mehr am Punkt der höchsten Übergangstemperatur, sondern periodisch (mit der Schichtdicke) links bzw. rechts von diesem optimalen Punkt. Entsprechend der Schärfe der Grenzflächen und abhängig von der Substratorientierung verkleinert sich die Übergangstemperatur für die verschiedenen Übergitter. Die Modulation des $e_{g}$-Bandes kann bei scharfen Grenzflächen auch entsprechend scharf sein: In L/SMO(001) ist bereits bei $n \geq 3$ die $e_{g}$-Elektronenanzahl in dem SMO gering genug, sodass SMO 
ein antiferromagnetisches Verhalten aufweist. In den Übergitter-Systemen auf STO(111) kann in dem Bereich $n \leq 2$ ein leichte Erhöhung der Übergangstemperatur registriert werden. Insbesondere zeigt das L/SMO(111) Übergitter mit $T_{C} \sim 365.5 \mathrm{~K}$ eine erhöhte Übergangstemperatur, die für keine Dotierung im LSMO Phasendiagramm erreicht wird. Obwohl die genaue Struktur dieses Übergitters nicht ermittelt werden kann, ist die A-PlatzKationen-Modulation durch XRR klar festzustellen. Offensichtlich ist die $e_{g}$-ElektronenModulation in (111) Übergittern für $n \leq 4$, aufgrund des ununterscheidbaren $T_{C}(n)$ Verlaufes von L/SMO(111) und LS/LMO(111), nicht so stark, wie in (001) Übergittern. Somit kann konstatiert werden, dass in A-Platz-Kation-geordneten/modulierten LSMO Filme, in denen die $e_{g}$-Elektronen-Modulation klein genug ist, die Modulation der Gitterverspannung, zu einer Erhöhung der Übergangstemperatur führen kann, wie es bereits durch andere Arbeiten $[7,8]$ postuliert wurde. Diese kleine Erhöhung der Übergangstemperatur ist natürlich nicht ausreichend um spintronische Anwendungen auf Perowskit-Basis bei Raumtemperatur, wie z.B. Tunnel-Magnetowider-stands-Elemente [5], zu verbessern. Jedoch wurde aufgezeigt, dass es möglich ist die Übergangs-temperatur, durch eine künstliche Gitter-Modulation über die A-Platz Ordnung, in LSMO zu erhöhen. Für zukünftige Experimente in diesem Bereich sollten Komponenten des Übergitters gewählt werden, die eine gleiche Valenz aufzeigen, damit man keine $e_{g}$-Elektronen-Modulation erhält, die der Erhöhung der Übergangstemperatur entgegen wirkt.

Ferrimagnetische, halbmetallische Doppelperowskite stellen, durch ihre hohen Curie-Temperaturen $[11,120]$, eine gute Alternative zu LSMO in perowskitischen, spintronischen Anwendungen dar. Diese Materialklasse zeigt zwei essentielle Herausforderungen bei der Präparation als dünner Film: Zum einen muss die B-Platz Ordnung in einer Kochsalzstruktur realisiert werden, zum anderen müssen die B-Platz Ionen die richtige Valenz aufweisen. Beides bedingt sich gegenseitig und kann über die Wahl der richtigen Depositionsbedingungen umgesetzt werden. Innerhalb dieser Arbeit wurden die zwei angesprochenen Herausforderungen erfolgreich mit der MAD Technologie bewältigt:

Die Untersuchung der natürliche B-Platz-Ordnung von MAD-präparierten Doppelperowskiten wurde an $\mathrm{La}_{2} \mathrm{CoMnO}_{6}$ durchgeführt. LMCO ist ein ferromagnetisches, isolierendes Doppelperowskit, welches, durch die benötigten Valenz-Zustände von $M n$ und $C o$, bei StandardMAD-Bedingungen (insbesondere in der Umgebungsatmosphäre) präpariert werden kann. Es ist gelungen $\sim 320 \mathrm{~nm}$ dicke Filme auf $\mathrm{STO}(111)$ zu präparieren, die eine B-Platz-Ordnung von $p>92$ - 98\% zeigen. Diese Abschätzung wurde über die Sättigungsmagnetisierung der Filme durchgeführt, nachdem dargelegt werden konnte, dass die Bestimmung der Ordnung über strukturelle Messmethoden (XRD, TEM), durch die Anwesenheit von Antiphasen- 
Grenzen, gestört wird. Die B-Platz-Ordnung konnte durch TEM-EELS Messungen lokal verifiziert und die B-Platz Valenz-Zustände in der geordneten Phase auf $\mathrm{Co}^{2+}$ und $\mathrm{Mn}^{4+}$, in Übereinstimmung mit der Literatur [127-132], bestimmt werden. Die Größe der vollständig geordneten Bereiche, bzw. der Abstand zwischen zwei Antiphasen-Grenzen, konnte über die TEM Investigation und über die magnetischen Eigenschaften auf eine Größen-ordnung von $\sim 100 \mathrm{~nm}$ abgeschätzt werden. Während $L a$-stöchiometrische Proben nach einer zusätzlichen thermalen Auslagerung eine hohe B-Platz-Ordnung zeigen, ist diese für La-defizitäre Proben bereits direkt nach der Deposition vorhanden. Dabei zeigt die Agglomeration der LaLeerstellen und überschüssigem $C o$, dass eine große Beweglichkeit in diesem Material vorhanden ist, welche die B-Platz-Ordnung unterstützt.

Eine Valenz-Kontrolle der Metall-Ionen wurde für die MAD-Technologie durch die Kontrolle des Sauerstoffpartialdruckes während der Deposition realisiert. Dabei wird der Sauerstoffpartialdruck über die Zufuhr von Sauerstoff und der Sauerstoff-bindenden metallorganischen Präkursorlösung reguliert. Diese neue LOMAD-Methode ermöglicht die Präparation von Oxiden, wie $\mathrm{Fe}_{3} \mathrm{O}_{4}$ und $\mathrm{VO}_{2}$, die mit der Standard-MAD-Methode, aufgrund des hohen Sauerstoffpartialdruckes, überoxidiert als $\mathrm{Fe}_{2} \mathrm{O}_{3}$ und $\mathrm{V}_{2} \mathrm{O}_{5}$ vorliegen würden. Insbesondere konnte gezeigt werden, dass mit der LOMAD-Methode glatte, epitaktische, qualitativ hochwertige Magnetit Filme gewachsen werden können. Erste Vorversuche zur Präparation von $\mathrm{Sr}_{2} \mathrm{FeMoO}_{6}$, einem ferrimagnetischen, halbmetallischen Doppelperowskit, konnten erfolgreich durchgeführt werden: Es wurden die genauen Präparationsbedingungen gefunden die zu einer Deposition von teilgeordneten SFMO, mit einer Curie-Temperatur von $T_{C} \sim 420 K$, führen. Es konnte festgestellt werden, dass eine weitere Optimierung der SFMO Film-Qualität, durch eine weitere Modifizierung der MAD Technologie hinsichtlich einer genaueren Regulierung des Sauerstoffpartialdruckes, erreicht werden kann. Zurzeit befindet sich eine neue MAD-Anlage in der Entwicklung, welche die genannten Modifikationen enthält und somit, mit Hilfe dieser Vorarbeit, die Präparation von qualitativ hochwertigen SFMO Filmen über das MAD-Prinzip ermöglicht.

Zusammenfassend kann in dieser Arbeit festgestellt werden, dass diverse künstlich oder natürlich Kationen-geordnete Perowskite mit der MAD-Technologie präpariert werden können. Die lagenweise A-Platz-Ordnung in Manganaten führt, über die Modulation der Gitterverspannung und der Elektronenbesetzung im $e_{g}$-Band der Manganionen, zu modifizierten elektronischen und magnetischen Eigenschaften. Es konnte ein Weg aufgezeigt werden, der zu Manganaten mit Übergangstemperaturen $T_{C}>370 K$ führen kann. In Doppelperowskiten ist die B-Platz-Ordnung essentiell für das Vorhandensein einer hoch-Temperatur ferri/ferromagnetischen Phase. Innerhalb dieser Arbeit wurde der Einfluss und die Anwe- 
senheit von Antiphasen-Grenzen in dem ferromagnetischen Doppelperowskit $\mathrm{La}_{2} \mathrm{CoMnO}_{6}$ dargelegt. Es konnte aufgezeigt werden, dass mit der ökonomischen MAD-Technologie, halbmetallische Doppelperowskite gewachsen werden können, die ein vielversprechendes Material für den Einsatz in spintronischen Anwendungen darstellen. 



\section{Literatur}

[1] D.W. Bruce, D. O'Hare, and R.I. Walton. Functional Oxides. Wiley, 2010.

[2] R. von Helmolt, J. Wecker, B. Holzapfel, L. Schultz, and K. Samwer. Giant negative magnetoresistance in perovskitelike $\mathrm{La}_{2 / 3} \mathrm{Ba}_{1 / 3} M n \mathrm{O}_{x}$ ferromagnetic films. Phys. Rev. Lett., 71:2331-2333, Oct 1993.

[3] J.G. Bednorz and K.A. Müller. Possible high $T_{c}$ superconductivity in the BaLaCuO system. Zeitschrift für Physik B Condensed Matter, 64(2):189-193, 1986.

[4] E. Dagotto. Open questions in cmr manganites, relevance of clustered states and analogies with other compounds including the cuprates. New Journal of Physics, 7(1):67, 2005.

[5] Y. Ogimoto, M. Izumi, A. Sawa, T. Manako, H. Sato, H. Akoh, M. Kawasaki, and Y. Tokura. Tunneling Magnetoresistance above Room Temperature in $\mathrm{La}_{0.7} \mathrm{Sr}_{0.3} \mathrm{MnO}_{3} / \mathrm{SrTiO}_{3} / \mathrm{La}_{0.7} \mathrm{Sr}_{0.3} \mathrm{MnO}_{3}$ Junctions. Japanese Journal of Applied Physics, 42(4A):L369, 2003.

[6] F. M. Postma, R. Ramaneti, T. Banerjee, H. Gokcan, E. Haq, D. H. A. Blank, R. Jansen, and J. C. Lodder. Epitaxial diodes of a half-metallic ferromagnet on an oxide semiconductor. Journal of Applied Physics, 95(11):7324-7326, 2004.

[7] L. M. Rodriguez-Martinez and J. P. Attfield. Cation disorder and size effects in magnetoresistive manganese oxide perovskites. Phys. Rev. B, 54:R15622-R15625, 1996.

[8] V. Moshnyaga, L. Sudheendra, O. I. Lebedev, S. A. Köster, K. Gehrke, O. Shapoval, A. Belenchuk, B. Damaschke, G. van Tendeloo, and K. Samwer. A-Site Ordering versus Electronic Inhomogeneity in Colossally Magnetoresistive Manganite Films. Phys. Rev. Lett., 97:107205, 2006.

[9] S. J. May, P. J. Ryan, J. L. Robertson, J.-W. Kim, T. S. Santos, E. Karapetrova, J. L. Zarestky, X. Zhai, S. G. E. Te Velthuis, J. N. Eckstein, S. D. Bader, and A. Bhattacharya. Enhanced ordering temperatures in antiferromagnetic manganite superlattices. Nature materials, 8(11):892-897, 2009.

[10] R. I. Dass and J. B. Goodenough. Multiple magnetic phases of $\mathrm{La}_{2} \mathrm{CoMnO}_{6}$. Phys. Rev. B, 67:014401, 2003.

[11] D. Serrate, J. M. De Teresa, and M. R. Ibarra. Double perovskites with ferromagnetism above room temperature. Journal of Physics: Condensed Matter, 19(2):023201, 2007.

[12] S. Mukhopadhyay and I. Das. Colossal enhancement of magnetoresistance in $\mathrm{La}_{0.67} \mathrm{Sr}_{0.33} \mathrm{MnO}_{3} / \mathrm{Pr}_{0.67} \mathrm{Ca}_{0.33} \mathrm{MnO}_{3}$ multilayers: Reproducing the phase separation scenario. EPL (Europhysics Letters), 83(2):27003, 2008.

[13] A. Sidorenko P.A. Petrenko A.Weidinger M. Zeitler B. Rauschenbach R. Tidecks K. Samwer V. Moshnyaga, I. Koroshun. Preparation of rare-earth manganite-oxide thin films by metalorganic aerosol deposition technique. Appl. Phys. Lett., 74:2842, 1999.

[14] U. Müller. Inorganic Structural Chemistry. Wiley \& Sons Ltd., 1993.

[15] Y. Tokura. Colossal magnetoresistive oxides. Gordon and Breach Science, 2000. 


\section{Literatur}

[16] Y. Konishi, Z. Fang, M. Izumi, T. Manako, M. Kasai, H. Kuwahara, M. Kawasaki, K. Terakura, and Y. Tokura. Orbital-State-Mediated Phase-Control of Manganites. Journal of the Physical Society of Japan, 68(12):3790-3793, 1999.

[17] Z. Fang, I. V. Solovyev, and K. Terakura. Phase Diagram of Tetragonal Manganites. Phys. Rev. Lett., 84:3169-3172, 2000.

[18] A. Tebano, C. Aruta, S. Sanna, P. G. Medaglia, G. Balestrino, A. A. Sidorenko, R. De Renzi, G. Ghiringhelli, L. Braicovich, V. Bisogni, and N. B. Brookes. Evidence of orbital reconstruction at interfaces in ultrathin $\mathrm{La}_{0.67} \mathrm{Sr}_{0.33} \mathrm{MnO}_{3}$ films. Physical review letters, 100(13):137401, 2008.

[19] J.M.D. Coey. Magnetism and Magnetic Materials. Cambridge University Press, 2004.

[20] H. A. Kramers. L'interaction entre les atomes magnétogènes dans un cristal paramagnétique. Physica 1, pages 182-192, 1934 .

[21] P. W. Anderson. Antiferromagnetism. Theory of Superexchange Interaction. Phys. Rev., 79:350-356, 1950.

[22] J. B. Goodenough. Theory of the Role of Covalence in the Perovskite-Type Manganites [La, M(II)]MnO 3 . Phys. Rev., 100:564-573, 1955.

[23] J. B. Goodenough. An interpretation of the magnetic properties of the perovskite-type mixed crystals $L a_{1-x} \mathrm{Sr}_{x} \mathrm{CoO}_{3-\lambda}$. Journal of Physics and Chemistry of Solids, 6(2-3):287 - 297, 1958.

[24] C. Zener. Interaction between the $d$-Shells in the Transition Metals. II. Ferromagnetic Compounds of Manganese with Perovskite Structure. Phys. Rev., 82:403-405, 1951.

[25] P. W. Anderson and H. Hasegawa. Considerations on Double Exchange. Phys. Rev., 100:675-681, 1955 .

[26] P. G. de Gennes. Effects of Double Exchange in Magnetic Crystals. Phys. Rev., 118:141-154, 1960.

[27] G. H. Jonker and Van Santen J. H. Ferromagnetic compounds of manganese with perovskite structure. Physica, 16(3):337 - 349, 1950.

[28] J.H. Van Santen and G.H. Jonker. Electrical conductivity of ferromagnetic compounds of manganese with perovskite structure. Physica, 16(7-8):599 - 600, 1950.

[29] A. Tebano, C. Aruta, P. G. Medaglia, F. Tozzi, G. Balestrino, A. A. Sidorenko, G. Allodi, R. De Renzi, G. Ghiringhelli, C. Dallera, L. Braicovich, and N. B. Brookes. Strain-induced phase separation in $\mathrm{La}_{0.7} \mathrm{Sr}_{0.3} \mathrm{MnO}_{3}$ thin films. Phys. Rev. B, 74:245116, 2006.

[30] V. M. Goldschmidt. Die Gesetze der Krystallochemie. Naturwissenschaften, 14:477-485, 1926.

[31] A. Urushibara, Y. Moritomo, T. Arima, A. Asamitsu, G. Kido, and Y. Tokura. Insulator-metal transition and giant magnetoresistance in $L a_{1-x} S_{x} M n O_{3}$. Phys. Rev. B, 51:14103-14109, 1995.

[32] M. Jungbauer, S. Hühn, R. Egoavil, H. Tan, J. Verbeeck, G. Van Tendeloo, and V. Moshnyaga. Atomic layer epitaxy of Ruddlesden-Popper $\mathrm{SrO}(\mathrm{SrTiO} 3) \mathrm{n}$ films by means of metalorganic aerosol deposition. Applied Physics Letters, 105(25):251603, 2014. 
[33] H. Fujiwara. Spectroscopic ellipsometry - principles and applications. John Wiley and Sons Ltd., 2007.

[34] S. N. Jasperson and S. E. Schnatterly. An Improved Method for High Reflectivity Ellipsometry Based on a New Polarization Modulation Technique. Review of Scientific Instruments, 40(6):761-767, 1969.

[35] M. J. Weber. Handbook of Laser Science and Technology Volume IV Optical Materials: Part 2: Properties. CRC Press, Inc., 1986.

[36] M. Jungbauer. Dissertation, Georg-August-Universität Göttingen, 2015.

[37] F. Chen, H. Lu, T. Zhao, K. Jin, Z. Chen, and G. Yang. Real-time optical monitoring of the heteroepitaxy of oxides by an oblique-incidence reflectance difference technique. Physical Review B, 61(15):10404, 2000.

[38] J.-T. Zettler, T. Wethkamp, M. Zorn, M. Pristovsek, C. Meyne, K. Ploska, and W. Richter. Growth oscillations with monolayer periodicity monitored by ellipsometry during metalorganic vapor phase epitaxy of GaAs(001). Applied Physics Letters, 67(25):3783-3785, 1995.

[39] J.-S. Lee and Y. Masumoto. Real-time monitoring of ellipsometry monolayer oscillations during metalorganic vapor-phase epitaxy. Journal of Crystal Growth, 221(1-4):111 - 116, 2000.

[40] M. Kawasaki, K. Takahashi, T. Maeda, R. Tsuchiya, M. Shinohara, O. Ishiyama, T. Yonezawa, M. Yoshimoto, and H. Koinuma. Atomic control of the $\mathrm{SrTiO}_{3}$ crystal surface. Science, 266(5190):1540-1542, 1994.

[41] G. Koster, B. L. Kropman, G. J. H. M. Rijnders, D. H. A. Blank, and H. Rogalla. Quasi-ideal strontium titanate crystal surfaces through formation of strontium hydroxide. Applied Physics Letters, 73(20):2920-2922, 1998.

[42] A. Biswas, P. B. Rossen, C.-H. Yang, W. Siemons, M.-H. Jung, I. K. Yang, R. Ramesh, and Y. H. Jeong. Universal Ti-rich termination of atomically flat $\mathrm{SrTiO}_{3}$ (001),(110), and (111) surfaces. Applied Physics Letters, 98(5):051904, 2011.

[43] R. Wiesendanger. Scanning Probe Microscopy and Spectroscopy - Methods and Applications. Cambridge University Press, 2010.

[44] D. Nečas and P. Klapetek. Gwyddion: an open-source software for SPM data analysis. Central European Journal of Physics, 10:181-188, 2012.

[45] B. D. Cullity and S. R. Stock. Elements of X-ray Diffraction. Prentice Hall, 2001.

[46] E. Prince. International Tables for Crystallography, Volume C: Mathematical, Physical and Chemical Tables, volume 3, page 554. Springer, 2004.

[47] M. Born and E. Wolf. Principles of Optics: Electromagnetic Theory of Propagation, Interference and Diffraction of Light. Cambridge University Press, 1999.

[48] L. G. Parratt. Surface Studies of Solids by Total Reflection of X-Rays. Phys. Rev., 95:359-369, 1954. 


\section{Literatur}

[49] L. G. Parratt and C. F. Hempstead. Anomalous dispersion and scattering of x-rays. Phys. Rev., 94:1593-1600, 1954.

[50] C. T. Chantler. Theoretical Form Factor, Attenuation and Scattering Tabulation for Z=1-92 from $\mathrm{E}=1-10 \mathrm{eV}$ to $\mathrm{E}=0.4-1.0 \mathrm{MeV}$. Journal of Physical and Chemical Reference Data, 24:71-643, 1995.

[51] S. Macke, S. Brück, P. Audehm, M. Harlander, and E. Goering. ReMagX: X-ray Magnetic Reflectivity Tool. Max-Planck Institute for Metals Research, 2009. www.mf.mpg.de/remagx.html.

[52] L. Nevot and P. Croce. Caractérisation des surfaces par réflexion rasante de rayons X. Application à l'étude du polissage de quelques verres silicates. Revue de Physique appliquée, 15(3):761-779, 1980.

[53] R. Egoavil. STEM investigation of complex oxides at atomic scale. Dissertation, EMAT, University of Antwerp, 2015.

[54] S. J. Pennycook and P. D. Nellist. Scanning Transmission Electron Microscopy. Springer, 2011.

[55] P. Hartel, H. Rose, and C. Dinges. Conditions and reasons for incoherent imaging in STEM. Ultramicroscopy, 63(2):93 - 114, 1996.

[56] R. F. Egerton. Electron Energy-Loss Spectroscopy in the Electron Microscope. Springer, 2011.

[57] L. J. Allen, A. J. D'Alfonso, B. Freitag, and D. O. Klenov. Chemical mapping at atomic resolution using energy-dispersive x-ray spectroscopy. MRS bulletin, 37(01):47-52, 2012.

[58] J. R. Ferraro, K. Nakamoto, and C. W. Brown. Introductory Raman Spectroscopy. Academic Press, 2003.

[59] W. Buckel and R. Kleiner. Supraleitung: Grundlagen und Anwendungen. Wiley-VCH Verlag GmbH und Co. KGaA, 2012.

[60] J. Clarke and A. I. Braginski. The SQUID Handbook, Vol.1: Fundamentals and Technology of SQUIDs and SQUID Systems. Wiley-VCH Verlag GmbH und Co. KGaA, 2004.

[61] J. Kerr. Rept. Brit. Assoc. Adv. Sci., 5:85, 1876.

[62] J. Kerr. On rotation of the plane of polarization by reflection from the pole of a magnet. Phil. Mag., $3: 321,1877$.

[63] A. D. Buckingham and P. J. Stephens. Magnetic optical activity. Annual Review of Physical Chemistry, 17(1):399-432, 1966.

[64] P. Bruno, Y. Suzuki, and C. Chappert. Magneto-optical kerr effect in a paramagnetic overlayer on a ferromagnetic substrate: A spin-polarized quantum size effect. Phys. Rev. B, 53:9214-9220, 1996.

[65] K. Chahara, T. Ohno, M. Kasai, and Y. Kozono. Magnetoresistance in magnetic manganese oxide with intrinsic antiferromagnetic spin structure. Applied Physics Letters, 63(14):1990-1992, 1993.

[66] J.-H. Park, E. Vescovo, H.-J. Kim, C. Kwon, R. Ramesh, and T. Venkatesan. Direct evidence for a half-metallic ferromagnet. Nature, 392(6678):794-796, 1998. 
[67] M. Imada, A. Fujimori, and Y. Tokura. Metal-insulator transitions. Rev. Mod. Phys., 70:1039-1263, 1998.

[68] E. O. Wollan and W. C. Koehler. Neutron diffraction study of the magnetic properties of the series of perovskite-type compounds $[(1-x) L a, x C a] \mathrm{MnO}_{3}$. Phys. Rev., 100:545-563, 1955.

[69] J. B. Goodenough, A. Wold, R. J. Arnott, and N. Menyuk. Relationship between crystal symmetry and magnetic properties of ionic compounds containing $\mathrm{Mn}^{3+}$. Phys. Rev., 124:373-384, 1961.

[70] E. Dagotto. Complexity in strongly correlated electronic systems. Science, 309(5732):257-262, 2005.

[71] A. Millis, P. Littlewood, and B. Shraiman. Double Exchange Alone Does Not Explain the Resistivity of $\mathrm{La}_{1_{x}} \mathrm{Sr}_{x} \mathrm{MnO}_{3}$. Phys. Rev. Lett., 74:5144-5147, 1995.

[72] A. Alexandrov and A. Bratkovsky. Carrier Density Collapse and Colossal Magnetoresistance in Doped Manganites. Phys. Rev. Lett., 82:141-144, Jan 1999.

[73] Neil Mathur and Peter Littlewood. Mesoscopic texture in manganites. Physics Today, 56(1):25-30, 2003.

[74] H. Hwang, S-W. Cheong, P. Radaelli, M. Marezio, and B. Batlogg. Lattice Effects on the Magnetoresistance in Doped $\mathrm{LaMnO}_{3}$. Phys. Rev. Lett., 75:914-917, 1995.

[75] V. Moshnyaga and K. Samwer. Electron-lattice correlations and phase transitions in CMR manganites. Annalen der Physik, 523(8-9):652-663, 2011.

[76] M. Uehara, S. Mori, C. H. Chen, and S.-W. Cheong. Percolative phase separation underlies colossal magnetoresistance in mixed-valent manganites. Nature, 399(6736):560-563, 1999.

[77] Sebastian Hühn. Präparation und Eigenschaften von dünnen $\left(\mathrm{La}_{1-y} \mathrm{Pr}_{y}\right)_{0.67} \mathrm{Ca}_{0.33} \mathrm{MnO} \mathrm{O}_{3}$ Schichten, 2010.

[78] V. Moshnyaga, A. Belenchuk, S. Hühn, C. Kalkert, M. Jungbauer, O. I. Lebedev, S. Merten, K.-Y. Choi, P. Lemmens, B. Damaschke, and K. Samwer. Intrinsic antiferromagnetic coupling underlies colossal magnetoresistance effect: Role of correlated polarons. Phys. Rev. B, 89:024420, 2014.

[79] V. Moshnyaga, K. Gehrke, O. Lebedev, L. Sudheendra, A. Belenchuk, S. Raabe, O. Shapoval, J. Verbeeck, G. Van Tendeloo, and K. Samwer. Electrical nonlinearity in colossal magnetoresistance manganite films: Relevance of correlated polarons. Phys. Rev. B, 79:134413, 2009.

[80] C. P. Bean and J. D. Livingston. Superparamagnetism. Journal of Applied Physics, 30:S120-S129, 1959.

[81] S. S. P. Parkin, R. Bhadra, and K. P. Roche. Oscillatory magnetic exchange coupling through thin copper layers. Phys. Rev. Lett., 66:2152-2155, 1991.

[82] W. Pan, N.-Y. Jih, C.-C. Kuo, and M.-T. Lin. Coercivity enhancement near blocking temperature in exchange biased $\mathrm{Fe} / \mathrm{Fe}_{x} \mathrm{Mn}(1-x)$ films on $\mathrm{Cu}(001)$. Journal of Applied Physics, 95(11):7297-7299, 2004 . 


\section{Literatur}

[83] K. R. Nikolaev, A. Yu. Dobin, I. N. Krivorotov, W. K. Cooley, A. Bhattacharya, A. L. Kobrinskii, L. I. Glazman, R. M. Wentzovitch, E. Dan Dahlberg, and A. M. Goldman. Oscillatory Exchange Coupling and Positive Magnetoresistance in Epitaxial Oxide Heterostructures. Phys. Rev. Lett., 85:3728-3731, 2000.

[84] C. Adams, J. Lynn, Y. Mukovskii, A. Arsenov, and D. Shulyatev. Charge Ordering and Polaron Formation in the Magnetoresistive Oxide $\mathrm{La}_{0.7} \mathrm{Ca}_{0.3} \mathrm{MnO}_{3}$. Phys. Rev. Lett., 85:3954-3957, 2000.

[85] P. Dai, J. Fernandez-Baca, N. Wakabayashi, E. Plummer, Y. Tomioka, and Y. Tokura. Short-Range Polaron Correlations in the Ferromagnetic $\mathrm{La}_{1-x} \mathrm{Ca} a_{x} M n \mathrm{O}_{3}$. Phys. Rev. Lett., 85:2553-2556, 2000.

[86] P. Schiffer, A. P. Ramirez, W. Bao, and S-W. Cheong. Low Temperature Magnetoresistance and the Magnetic Phase Diagram of $L a_{1-x} C a_{x} M n O_{3}$. Phys. Rev. Lett., 75:3336-3339, 1995.

[87] Y. Tomioka, A. Asamitsu, H. Kuwahara, Y. Moritomo, and Y. Tokura. Magnetic-field-induced metalinsulator phenomena in $\mathrm{Pr}_{1-x} \mathrm{Ca}_{x} \mathrm{MnO}_{3}$ with controlled charge-ordering instability. Phys. Rev. B, 53:R1689-R1692, 1996.

[88] Z. Jirák, S. Krupička, Z. Šimša, M. Dlouhá, and S. Vratislav. Neutron diffraction study of $\mathrm{Pr}_{1-x} \mathrm{Ca}_{x} \mathrm{MnO}_{3}$ perovskites. Journal of Magnetism and Magnetic Materials, 53(1-2):153-166, 1985.

[89] D. E. Cox, P. G. Radaelli, M. Marezio, and S-W. Cheong. Structural changes, clustering, and photoinduced phase segregation in $\mathrm{Pr}_{0.7} \mathrm{Ca}_{0.3} \mathrm{MnO}_{3}$. Phys. Rev. B, 57:3305-3314, Feb 1998.

[90] J. L. Garcia-Munoz, A. Collado, M. A. G. Aranda, and C. Ritter. Multilevel hierarchy of phase separation processes in $\mathrm{La}_{5 / 8-y} \mathrm{Pr}_{y} \mathrm{Ca}_{3 / 8} \mathrm{MnO}_{3}$. Phys. Rev. B, 84:024425, 2011.

[91] H. Fujishiro, T. Fukase, and M. Ikebe. Anomalous Lattice Softening at $X=0.19$ and 0.82 in $L a_{1-X} C a_{X} M n O_{3}$. Journal of the Physical Society of Japan, 70(3):628-631, 2001.

[92] J. Narayan and B. C. Larson. Domain epitaxy: A unified paradigm for thin film growth. Journal of Applied Physics, 93(1):278-285, 2003.

[93] E. S. Vlakhov, R. A. Chakalov, R. I. Chakalova, K. A. Nenkov, K. Dörr, A. Handstein, and K.-H. Müller. Influence of the substrate on growth and magnetoresistance of $L a_{0.7} C a_{0.3} M n O_{z}$ thin films deposited by magnetron sputtering. Journal of Applied Physics, 83(4):2152-2157, 1998.

[94] Z. Jirák, S. Krupička, Z. Šimša, M. Dlouhá, and S. Vratislav. Neutron diffraction study of $\mathrm{Pr}_{1-x} \mathrm{Ca}_{x} \mathrm{MnO}_{3}$ perovskites. Journal of Magnetism and Magnetic Materials, 53(1-2):153 - 166, 1985.

[95] Z. Q. Yang, R. W. A. Hendrikx, P. J. M. v. Bentum, and J. Aarts. Disorder-induced melting of the charge order in thin films of $\operatorname{Pr}_{0.5} \mathrm{Ca}_{0.5} \mathrm{MnO}_{3}$. EPL (Europhysics Letters), 58(6):864, 2002.

[96] Y. Tomioka, A. Asamitsu, Y. Moritomo, and Y. Tokura. Anomalous Magnetotransport Properties of $\mathrm{Pr}_{1-x} \mathrm{Ca}_{x} \mathrm{MnO}_{3}$. Journal of the Physical Society of Japan, 64(10):3626-3630, 1995.

[97] Ch. Jooss, L. Wu, T. Beetz, R. F. Klie, M. Beleggia, M. A. Schofield, S. Schramm, J. Hoffmann, and Y. Zhu. Polaron melting and ordering as key mechanisms for colossal resistance effects in manganites. Proceedings of the National Academy of Sciences, 104(34):13597-13602, 2007. 
[98] Felix Massel. Diffusionsversuche an dünnen Lanthan-Calcium-Manganat/Praseodym-CalciumManganat-Multilagen, 2011.

[99] Felix Koeth. Optische Eigenschaften dünner LPCMO-Schichten, 2011.

[100] P. Levy, F. Parisi, M. Quintero, L. Granja, J. Curiale, J. Sacanell, G. Leyva, G. Polla, R. S. Freitas, and L. Ghivelder. Nonvolatile magnetoresistive memory in phase separated $L a_{0.325} P r_{0.300} C a_{0.375} M n O_{3}$. Phys. Rev. B, 65:140401, 2002.

[101] L. Ghivelder and F. Parisi. Dynamic phase separation in $L a_{5 / 8-y} \operatorname{Pr}_{y} C a_{3 / 8} M n O_{3}$. Phys. Rev. B, $71: 184425,2005$.

[102] L. Wu, R. F. Klie, Y. Zhu, and Ch. Jooss. Experimental confirmation of zener-polaron-type charge and orbital ordering in $\operatorname{Pr}_{1-x} \mathrm{Ca}_{x} \mathrm{MnO}_{3}$. Phys. Rev. B, 76:174210, 2007.

[103] R.D. Shannon. Revised effective ionic radii and systematic studies of interatomic distances in halides and chalcogenides. Acta Cryst., A32:751-767, 1976.

[104] Danny Schwarzbach. Wachstum von dünnen $\mathrm{La}_{0.7} \mathrm{Sr}_{0.3} \mathrm{MnO}_{3}$-Schichten auf verschieden orientierten $\mathrm{SrTiO}_{3}$-Substraten. Bachelorarbeit, Georg-August-Universität Göttingen, 2013.

[105] A. Bhattacharya, S. J. May, S. G. E. te Velthuis, M. Warusawithana, X. Zhai, Bin Jiang, J.-M. Zuo, M. R. Fitzsimmons, S. D. Bader, and J. N. Eckstein. Metal-Insulator Transition and Its Relation to Magnetic Structure in $\left(\mathrm{LaMnO}_{3}\right)_{2 n} /\left(\mathrm{SrMnO}_{3}\right)_{n}$ Superlattices. Phys. Rev. Lett., 100:257203, 2008.

[106] C. Adamo, X. Ke, P. Schiffer, A. Soukiassian, M. Warusawithana, L. Maritato, and D. G. Schlom. Electrical and magnetic properties of $\left(\mathrm{SrMnO}_{3}\right)_{n} /\left(\mathrm{LaMnO}_{3}\right)_{2 n}$ superlattices. Applied Physics Letters, 92(11):112508, 2008.

[107] S. Dong, R. Yu, S. Yunoki, G. Alvarez, J.-M. Liu, and E. Dagotto. Magnetism, conductivity, and orbital order in $\left(\mathrm{LaMnO}_{3}\right)_{2 n} /\left(\mathrm{SrMnO}_{3}\right)_{n}$ superlattices. Phys. Rev. B, 78:201102, 2008.

[108] A. Gupta, T. R. McGuire, P. R. Duncombe, M. Rupp, J. Z. Sun, W. J. Gallagher, and G. Xiao. Growth and giant magnetoresistance properties of La-deficient $\operatorname{LaxMnO}_{3-\delta}(0.67 \leq x \leq 1)$ films. Applied physics letters, 67(23):3494-3496, 1995.

[109] R. Zhao, K. Jin, Z. Xu, H. Guo, L. Wang, C. Ge, H. Lu, and G. Yang. The oxygen vacancy effect on the magnetic property of the $\mathrm{LaMnO}_{3-\delta}$ thin films. Applied Physics Letters, 102(12):122402, 2013.

[110] A. Bhattacharya, X. Zhai, M. Warusawithana, J. N. Eckstein, and S. D. Bader. Signatures of enhanced ordering temperatures in digital superlattices of $(\mathrm{LaMnO} 3)_{m} /(\mathrm{SrMnO} 3)_{2} m$. Applied physics letters, 90(22):222503-222503, 2007.

[111] O. Chmaissem, B. Dabrowski, S. Kolesnik, J. Mais, D. E. Brown, R. Kruk, P. Prior, B. Pyles, and J. D. Jorgensen. Relationship between structural parameters and the néel temperature in $\mathrm{Sr}_{1-x} \mathrm{Ca}_{x} \mathrm{MnO}_{3}$ $(0<x<1)$ and $\mathrm{Sr}_{1-y} \mathrm{Ba}_{y} \mathrm{MnO}_{3}(y<0.2)$. Phys. Rev. B, 64:134412, 2001.

[112] Danny Schwarzbach. Masterarbeit, Georg-August-Universität Göttingen, 2015. 


\section{Literatur}

[113] H. Boschker, M. Huijben, A. Vailionis, J. Verbeeck, S. van Aert, M. Luysberg, S. Bals, G. van Tendeloo, E. P. Houwman, G. Koster, D. H. A. Blank, and G. Rijnders. Optimized fabrication of high-quality $\mathrm{La}_{0.67} \mathrm{Sr}_{0.33} \mathrm{MnO}_{3}$ thin films considering all essential characteristics. Journal of Physics D: Applied Physics, 44(20):205001, 2011.

[114] J. Hemberger, A. Krimmel, T. Kurz, H.-A. Krug von Nidda, V. Yu. Ivanov, A. A. Mukhin, A. M. Balbashov, and A. Loidl. Structural, magnetic, and electrical properties of single-crystalline $\mathrm{La}_{1-x} \mathrm{Sr}_{x} \mathrm{MnO}_{3}(0.4<x<0.85)$. Phys. Rev. B, 66:094410, 2002.

[115] N. Nakagawa, H. Y. Hwang, and D. A. Muller. Why some interfaces cannot be sharp. Nature materials, 5(3):204-209, 2006.

[116] S. J. May, A. B. Shah, S. G. E. Te Velthuis, M. R. Fitzsimmons, J. M. Zuo, X. Zhai, J. N. Eckstein, S. D. Bader, and A. Bhattacharya. Magnetically asymmetric interfaces in a $\mathrm{LaMnO}_{3} / \mathrm{SrMnO}_{3}$ superlattice due to structural asymmetries. Physical Review B, 77(17):174409, 2008.

[117] M. Angeloni, G. Balestrino, N. G. Boggio, P. G. Medaglia, P. Orgiani, and A. Tebano. Suppression of the metal-insulator transition temperature in thin $\mathrm{La}_{0.7} \mathrm{Sr}_{0.3} \mathrm{MnO}_{3}$ films. Journal of Applied Physics, 96:6387-6392, December 2004.

[118] R. Dass, J.-Q. Yan, and J. Goodenough. Oxygen stoichiometry, ferromagnetism, and transport properties of $L a_{2-x} N i M n O_{6+\delta}$. Phys. Rev. B, 68:064415, 2003.

[119] M. P. Singh, K. Truong, and P. Fournier. Magnetodielectric effect in double perovskite $\mathrm{La}_{2} \mathrm{CoMnO}_{6}$ thin films. Appl. Phys. Lett., 91:042504, 2007.

[120] T. K. Mandal, C. Felser, M. Greenblatt, and J. Kübler. Magnetic and electronic properties of double perovskites and estimation of their curie temperatures by ab initio calculations. Physical Review B, 78(13):134431, 2008.

[121] G. Woodward and P. M. King. Cation ordering in perovskites. J. Mater. Chem. (20), 5785, 2010.

[122] J.B. Goodenough and R.I. Dass. Comment on the magnetic properties of the system $\mathrm{Sr}_{2-x} \mathrm{Ca}_{x} \mathrm{FeMoO}_{6}, 0 \leq x \leq 2$. International Journal of Inorganic Materials, 2(1):3 - 9, 2000.

[123] S. Yamaguchi, Y. Okimoto, and Y. Tokura. Local lattice distortion during the spin-state transition in $\mathrm{LaCoO}_{3}$. Phys. Rev. B, 55:R8666-R8669, 1997.

[124] D. Louca, J. L. Sarrao, J. D. Thompson, H. Röder, and G. H. Kwei. Correlation of local jahn-teller distortions to the magnetic/conductive states of $L a_{1-x} \mathrm{Sr}_{x} \mathrm{CoO}_{3}$. Phys. Rev. B, 60:10378-10382, 1999.

[125] J.-S. Zhou, H. Q. Yin, and J. B. Goodenough. Vibronic superexchange in single-crystal $L a M n_{1-x} G a_{x} O_{3}$. Phys. Rev. B, 63:184423, 2001.

[126] J. B. Goodenough, R. I. Dass, and J. Zhou. Spin-glass to ferromagnet transition in $L a M n_{1-x} S c_{x} O_{3}$. Solid State Sciences, 4(3):297 - 304, 2002.

[127] M. J. Krishna and A. Venimadhav. Reentrant cluster glass behavior in $\mathrm{La}_{2} \mathrm{CoMnO}_{6}$ nanoparticles. J. of Appl. Phys., 113:163906, 2013. 
[128] C. L. Bull, D. Gleeson, and K. S. Knight. Dertermination of B-Site ordering and structural transformations in the mixed transition metal perovskites $\mathrm{La}_{2} \mathrm{CoMnO}_{6}$ and $\mathrm{La}_{2} \mathrm{NiMnO}_{6}$. J. Phys. : Condens. Matter, 15:4927-4936, 2003.

[129] I. O. Troyanchuk, A. P. Sazonova, H. Szymczak, D. Többens, and H. Gamari-Seale. Phase Separation in $\mathrm{La}_{2-x} A_{x} \mathrm{CoMnO} \mathrm{O}_{6}(A=\mathrm{Ca}$ and $\mathrm{Sr})$ Perovskites. J. of Exp. and Theo. Phys., 99(2):363, 2004.

[130] T. Kyômen, R. Yamazaki, and M. Itoh. Correlation between Magnetic Properties and Mn/Co Atomic Order in $\mathrm{LaMn}_{0.5} \mathrm{Co}_{0.5} \mathrm{O}_{3+\delta}:$ 1. Second-Order Nature in $\mathrm{Mn} / \mathrm{Co}$ Atomic Ordering and Valence State. Chemistry of Materials, 15(25):4798-4803, 2003.

[131] T. Burnus, Z. Hu, H. H. Hsieh, V. L. J. Joly, P. A. Joy, M. W. Haverkort, Hua Wu, A. Tanaka, H.-J. Lin, C. T. Chen, and L. H. Tjeng. Local electronic structure and magnetic properties of $L a M n_{0.5} C_{0.5} O_{3}$ studied by x-ray absorption and magnetic circular dichroism spectroscopy. Phys. Rev. B, 77:125124, 2008 .

[132] V. L. J. Joly, Y. B. Khollam, P. A. Joy, C. S. Gopinath, and S. K. Date. Unusual charge disproportionation and associated magnetic behaviour in nanocrystalline $\mathrm{LaMn}_{0.5} \mathrm{Co}_{0.5} \mathrm{O}_{3}$. Journal of Physics: Condensed Matter, 13(48):11001, 2001.

[133] H. Wadati, D. G. Hawthorn, T. Z. Regier, M. P. Singh, K. D. Truong, P. Fournier, and G. A. Sawatzky. X-ray absorption studies of $\mathrm{La}_{2} \mathrm{CoMnO}_{6}$ and $\mathrm{La}_{2} \mathrm{NiMnO}_{6}$. Chemical and Materials Science, 49:114$115,2009$.

[134] G. H. Jonker. Magnetic and Semiconducting Properties of Perovskites Containing Manganese and Cobalt. Journal of Applied Physics, 37(3):1424-1430, 1966.

[135] T. Kyômen, R. Yamazaki, and M. Itoh. Correlation between Magnetic Properties and Mn/Co Atomic Order in $\mathrm{LaMn}_{0.5} \mathrm{Co}_{0.5} \mathrm{O}_{3+\delta}$ : 2. Magnetic and Calorimetric Properties. Chemistry of Materials, 16(1):179-184, 2004.

[136] M. P. Singh, S. Carpentier, K. Truong, and P. Fournier. Evidence of bidomain structure in doubleperovskite $\mathrm{La}_{2} \mathrm{CoMnO}_{6}$ thin films. Appl. Phys. Lett., 90:211915, 2007.

[137] H. Z. Guo, A. Gupta, T. G. Calvarese, and M. A. Subramanian. Structural and magnetic properties of epitaxial thin films of the ordered double perovskite $\mathrm{La}_{2} \mathrm{CoMnO} \mathrm{O}_{6}$. Applied Physics Letters, 89(26):262503, 2006.

[138] A. J. Barón-González, C. Frontera, J. L. García-Muñoz, J. Roqueta, and J. Santiso. Magnetic, structural properties and b-site order of two epitaxial $\mathrm{La}_{2} \mathrm{CoMnO}_{6}$ films with perpendicular out-of-plane orientation. Journal of Physics: Conference Series, 200(9):092002, 2010.

[139] S. Yamaguchi, Y. Okimoto, H. Taniguchi, and Y. Tokura. Spin-state transition and high-spin polarons in $\mathrm{LaCoO}_{3}$. Phys. Rev. B, 53:R2926-R2929, 1996.

[140] D. Fuchs, C. Pinta, T. Schwarz, P. Schweiss, P. Nagel, S. Schuppler, R. Schneider, M. Merz, G. Roth, and H. v. Löhneysen. Ferromagnetic order in epitaxially strained $\mathrm{LaCoO}_{3}$ thin films. Phys. Rev. B, 75:144402, 2007. 


\section{Literatur}

[141] Rata, A. D., Herklotz, A., Schultz, L., and Dörr, K. Lattice structure and magnetization of $\mathrm{LaCoO}_{3}$ thin films. Eur. Phys. J. B, 76(2):215-219, 2010.

[142] V. V. Mehta, M. Liberati, F. J. Wong, R. V. Chopdekar, E. Arenholz, and Y. Suzuki. Ferromagnetism in tetragonally distorted $\mathrm{LaCoO}_{3}$ thin films. Journal of Applied Physics, 105(7):07E503, 2009.

[143] P. Scherrer. Bestimmung der Größe und der inneren Struktur von Kolloidteilchen mittels Röntgenstrahlen. Nachrichten von der Gesellschaft der Wissenschaften zu Göttingen, MathematischPhysikalische Klasse, 1918:98-100, 1918.

[144] J. Longo and R. Ward. Magnetic Compounds of Hexavalent Rhenium with the Perovskite-type Structure. Journal of the American Chemical Society, 83(13):2816-2818, 1961.

[145] F. K. Patterson, C. W. Moeller, and R. Ward. Magnetic Oxides of Molybdenum(V) and Tungsten(V) with the Ordered Perovskite Structure. Inorganic Chemistry, 2(1):196-198, 1963.

[146] A. W. Sleight and J. F. Weiher. Magnetic and electrical properties of $B a_{2} M R e O_{6}$ ordered perovskites. Journal of Physics and Chemistry of Solids, 33(3):679 - 687, 1972.

[147] K.-I. Kobayashi, T. Kimura, H. Sawada, K. Terakura, and Y. Tokura. Room-temperature magnetoresistance in an oxide material with an ordered double-perovskite structure. Nature, 395(6703):677-680, 1998.

[148] F. S. Galasso, F. C. Douglas, and R. J. Kasper. Relationship between magnetic curie points and cell sizes of solid solutions with the ordered perovskite structure. The Journal of Chemical Physics, 44(4):1672-1674, 1966.

[149] Y. Tomioka, T. Okuda, Y. Okimoto, R. Kumai, K.-I. Kobayashi, and Y. Tokura. Magnetic and electronic properties of a single crystal of ordered double perovskite $\mathrm{Sr}_{2} \mathrm{FeMoO}_{6}$. Phys. Rev. B, 61:422-427, 2000.

[150] Y. Moritomo, N. Shimamoto, S. Xu, A. Machida, E. Nishibori, M. Takata, M. Sakata, and A. Nakamura. Effects of B-Site Disorder in $\mathrm{Sr}_{2} \mathrm{FeMoO}_{6}$ with Double Perovskite Structure. Japanese Journal of Applied Physics, 40(7A):L672, 2001.

[151] Ll. Balcells, J. Navarro, M. Bibes, A. Roig, Martínez B., and J. Fontcuberta. Cationic ordering control of magnetization in $\mathrm{Sr}_{2} \mathrm{FeMoO}_{6}$ double perovskite. Applied Physics Letters, 78(6):781-783, 2001.

[152] J. L. Alonso, L. A. Fernández, F. Guinea, F. Lesmes, and V. Martín-Mayor. Phase diagram and influence of defects in the double perovskites. Phys. Rev. B, 67:214423, 2003.

[153] J. Linden, T. Yamamoto, M. Karppinen, H. Yamauchi, and T. Pietari. Evidence for valence fluctuation of $\mathrm{Fe}$ in $\mathrm{Sr}_{2} \mathrm{FeMoO}_{6-w}$ double perovskite. Applied Physics Letters, 76(20):2925-2927, 2000.

[154] M. S. Moreno, J. E. Gayone, M. Abbate, A. Caneiro, D. Niebieskikwiat, R. D. Sanchez, A. De Siervo, R. Landers, and G. Zampieri. $\mathrm{Fe}$ and $\mathrm{Mo}$ Valences in $\mathrm{Sr}_{2} \mathrm{FeMoO}_{6}$. Solid state communications, 120(4):161-164, 2001.

[155] J. M. Greneche, M. Venkatesan, R. Suryanarayanan, and J. M. D. Coey. Mössbauer spectrometry of $A_{2} \mathrm{FeMoO}_{6}(a=\mathrm{Ca}, \mathrm{Sr}, \mathrm{Ba})$ : Search for antiphase domains. Phys. Rev. B, 63:174403, 2001. 
[156] C. Ritter, M. R. Ibarra, L. Morellon, J. Blasco, J. Garcia, and J. M. De Teresa. Structural and magnetic properties of double perovskites $A A^{\prime} \mathrm{FeMoO}_{6}\left(A A^{\prime}=B a_{2}, B a S r, S r_{2}\right.$ and $\left.\mathrm{Ca}_{2}\right)$. Journal of Physics: Condensed Matter, 12(38):8295, 2000.

[157] M. Besse, V. Cros, A. Barthélémy, H. Jaffrès, J. Vogel, F. Petroff, A. Mirone, A. Tagliaferri, P. Bencok, P. Decorse, et al. Experimental evidence of the ferrimagnetic ground state of $\mathrm{Sr}_{2} \mathrm{FeMoO}_{6}$ probed by X-ray magnetic circular dichroism. EPL (Europhysics Letters), 60(4):608, 2002.

[158] T. Koide, T. Sekine, H. Miyauchi, H. Manaka, D. Asakura, A. Fujimori, K. I. Kobayashi, Y. Tomioka, T. Kimura, and Y. Tokura. Microscopic origin of ferrimagnetism of a double perovskite $\mathrm{Sr}_{2} \mathrm{FeMoO}_{6}$ : An x-ray magnetic circular dichroism study. 502(1):012003, 2014.

[159] B. García-Landa, C. Ritter, M.R. Ibarra, J. Blasco, P.A. Algarabel, R. Mahendiran, and J. García. Magnetic and magnetotransport properties of the ordered perovskite $\mathrm{Sr}_{2} \mathrm{FeMoO}_{6}$. Solid State Communications, 110(8):435 - 438, 1999.

[160] O. Kubaschewski and E.L. Evans. Metallurgische Thermochemie. Verlag Technik, 1959.

[161] H. J. T. Ellingham. Transactions and Communications: Reducibility of oxides and sulphides in metallurgical processes. Journal of the Society of Chemical Industry, 63(5):125-160, 1944.

[162] T. Manako, M. Izumi, Y. Konishi, K.-I. Kobayashi, M. Kawasaki, and Y. Tokura. Epitaxial thin films of ordered double perovskite $\mathrm{Sr}_{2} \mathrm{FeMoO}$. Applied physics letters, 74(15):2215-2217, 1999.

[163] J. Santiso, A. Figueras, and J. Fraxedas. Thin films of $\mathrm{Sr}_{2} \mathrm{FeMoO}$ grown by pulsed laser deposition: preparation and characterization. Surface and interface analysis, 33(8):676-680, 2002.

[164] A. J. Hauser, R. E. A. Williams, R. A. Ricciardo, A. Genc, M. Dixit, J. M. Lucy, P. M. Woodward, H. L. Fraser, and F. Yang. Unlocking the potential of half-metallic $\mathrm{Sr}_{2} \mathrm{FeMoO}_{6}$ films through controlled stoichiometry and double-perovskite ordering. Physical Review B, 83(1):014407, 2011.

[165] D. Serrate, J. M. De Teresa, J. Blasco, L. Morellon, and M. R. Ibarra. Properties of half metallic $\left(\mathrm{Ba}_{0.8} \mathrm{Sr}_{0.2}\right)_{2-x} \mathrm{La}_{2 x / 3} \sharp_{x / 3} \mathrm{Fe} \mathrm{MoO}_{6}$ double perovskites. Journal of magnetism and magnetic materials, 290:1021-1024, 2005.

[166] H. Asano, S. B. Ogale, J. Garrison, A. Orozco, Y. H. Li, E. Li, V. Smolyaninova, C. Galley, M. Downes, M. Rajeswari, R. Ramesh, and T. Venkatesan. Pulsed-laser-deposited epitaxial $\mathrm{Sr}_{2} \mathrm{FeMoO}_{6-y}$ thin films: Positive and negative magnetoresistance regimes. Applied Physics Letters, 74(24):3696-3698, 1999 .

[167] M. Hanesch. Raman spectroscopy of iron oxides and (oxy)hydroxides at low laser power and possible applications in enviromental magnetic studies. Geophys. J. Int., 177:941-948, 2009.

[168] D. T. Margulies, F. T. Parker, F. E. Spada, R. S. Goldman, J. Li, R. Sinclair, and A. E. Berkowitz. Anomalous moment and anisotropy behavior in $\mathrm{Fe}_{3} \mathrm{O}_{4}$ films. Physical Review B, 53(14):9175, 1996.

[169] Victor Pfahl. Sauerstoffarme MAD: Struktur und Magnetotransport von Magnetitschichten, 2014.

[170] E. J. W. Verwey and P. W. Haayman. Electronic conductivity and transition point of magnetite $\mathrm{Fe}_{3} \mathrm{O}_{4}$. Physica, 8(9):979-987, 1941. 


\section{Literatur}

[171] D. D. Ebbing. In General Chemistry 3rd ed., page 222. Houghton Mifflin Company, Boston, MA, 1990.

[172] H. Yamada, M. Kawasaki, and Y. Tokura. Epitaxial growth and valence control of strained perovskite $\mathrm{SrFeO}_{3}$ films. Applied physics letters, 80(4):622-624, 2002.

[173] E. Gürmen, E. Daniels, and J. S. King. Crystal Structure Refinement of $\mathrm{SrMoO}_{4}, \mathrm{SrWO}_{4}, \mathrm{CaMoO}_{4}$, and $\mathrm{BaWO}_{4}$ by Neutron Diffraction. The Journal of Chemical Physics, 55(3):1093-1097, 1971.

[174] C. Brisi and P. Rolando. Ann. Chim. (Rome), 59:385-399, 1969.

[175] W. Meiklejohn and C. Bean. New Magnetic Anisotropy. Phys. Rev., 105:904-913, 1957.

[176] S. Middey, S. Jana, and S. Ray. Surface spin-glass and exchange bias in $\mathrm{Sr}_{2} \mathrm{FeMoO}_{6}$ nanoparticle. Journal of Applied Physics, 108(4):043918-043918, 2010. 


\section{Abbildungsverzeichnis}

1 Idealisierte Darstellung eines kubischen Perowskites $\ldots \ldots \ldots \ldots \ldots \ldots$

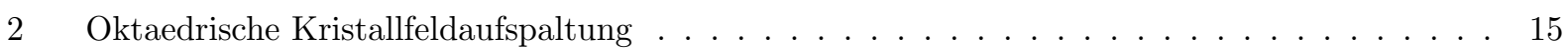

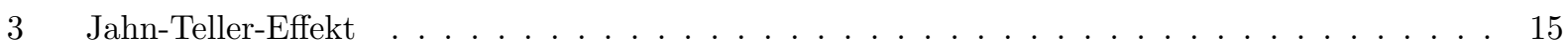

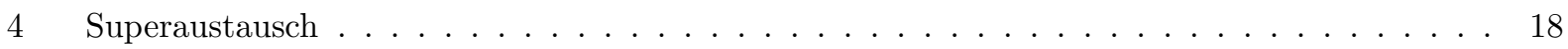

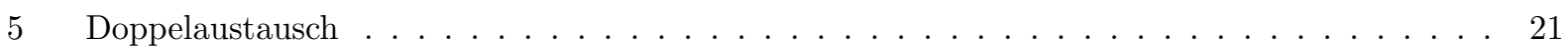

6 Schematischer Aufbau der MAD Anlagen . . . . . . . . . . . . . . . . . . . . . 28

$7 \quad$ SFM Aufnahmen von einem $\mathrm{STO}(001)$ und $\mathrm{STO}(111)$ Substrat $\ldots \ldots \ldots \ldots \ldots \ldots$. . . . . . 31

$8 \quad$ XRD-Messung der in dieser Arbeit verwendeten Substrate . . . . . . . . . . . . . . . 33

9 Magnetische Eigenschaften der STO und $\mathrm{MgO}$ Substrate $\ldots \ldots \ldots \ldots \ldots \ldots$

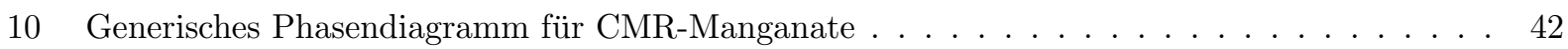

11 Elektronische und magnetische Eigenschaften von $\left(\mathrm{La}_{0.6} \mathrm{Pr}_{0.4}\right)_{0.67} C a_{0.33} \mathrm{MnO}_{3} \ldots \ldots \ldots$

12 Modell des CMR über die elektronische Phasenseparation . . . . . . . . . . . . . . . 47

13 Phasendiagramm von $\left(L a_{1-y} P r_{y}\right)_{0.67} C a_{0.33} M n O_{3}$ auf $\mathrm{MgO}(001) \ldots \ldots \ldots \ldots$

14 Strukturelle Daten von LCMO, PCMO Filmen und LCMO/PCMO Übergitter . . . . . . . . 51

15 Magnetische Eigenschaften von LCMO und PCMO auf $\mathrm{MgO}(001) \ldots \ldots \ldots \ldots$. . . . . 52

16 Abschätzung der Ladungsordnungstemperatur in PCMO . . . . . . . . . . . . . . . . . 52

17 XRR Messung und Simulation von LCMO/PCMO Übergittern auf $\mathrm{MgO}(001) \ldots \ldots \ldots$. . . . . 54

18 Elektronische Eigenschaften der LCMO/PCMO Übergitter auf $\mathrm{MgO}(001) \ldots \ldots \ldots$. . . . . 57

$19 M(H)$-Kurven der LCMO/PCMO Übergitter . . . . . . . . . . . . . . . . . . . 57

$20 M(T)$-Kurven der LCMO $/$ PCMO Übergitter $\ldots \ldots \ldots \ldots \ldots \ldots \ldots$

21 Relaxation des elektrischen Widerstands in LCMO/PCMO Übergittern . . . . . . . . . . . . 59

22 Vergleich der physikalischen Eigenschaften von LPCMO und LCMO/PCMO . . . . . . . . . . 63

23 Physikalische Eigenschaften von geordneten und ungeordneten $\mathrm{La}_{0.75} \mathrm{Ca}_{0.25} \mathrm{MnO}_{3} \ldots \ldots 8$

24 Elektrische und magnetische Eigenschaften von LMO und SMO auf STO(001) . . . . . . . . 71 
Abbildungsverzeichnis

STM Aufnahmen von LMO und SMO auf STO $(001) \ldots \ldots \ldots \ldots \ldots \ldots$. . . . . . . 72

XRD Daten von $\mathrm{LMO}$ und $\mathrm{SMO}$ auf $\mathrm{STO}(001) \ldots \ldots \ldots \ldots \ldots$

$27 M(T)$ und $R(T)$ Kurven für LSMO auf $\mathrm{STO}(001)$ ubd $\mathrm{STO}(111) \ldots \ldots . \ldots . \ldots 75$

28 Phasendiagramm für LSMO Filme auf $\mathrm{STO}(001)$ und $\mathrm{STO}(111) \ldots \ldots$. . . . . . . . . 76

29 Schichtdicken-Serie für LMO, SMO und LSMO . . . . . . . . . . . . . . . . . . . . 77

30 HAADF TEM Aufnahmen von LSMO Übergittern auf STO(111) . . . . . . . . . . . . . . . 79

31 Elementspezifische EELS TEM Aufnahmen von einem LSMO Übergitter auf STO(001) . . . 79

32 STM Aufnahmen von LSMO Übergittern . . . . . . . . . . . . . . . . . . . . . . . 80

33 XRR Messung und Simulation von LSMO Übergittern . . . . . . . . . . . . . . . . . . . . 82

34 XRD Messung und Simulation eines LMO/SMO Übergitters auf STO(001) . . . . . . . . . . 83

35 Temperaturabhängigkeit des spez. elektr. Widerstandes der L/SMO(111) Übergitter . . . . . 85

36 Magnetische Daten einiger LSMO Übergitter auf $\mathrm{STO}(001)$ und $\mathrm{STO}(111)$. . . . . . . . . 86

37 Zusammenfassung der magnetischen Eigenschaften der LSMO Übergitter . . . . . . . . . . . 88

38 Strukturelle und magnetische Daten von $\mathrm{LaCoO}_{3}$ auf $\mathrm{STO}(001) \quad \ldots \ldots$

39 Strukturelle, elektrische und magnetische Daten von $\mathrm{La}_{2} C o M n \mathrm{O}_{6}$ auf $\mathrm{STO}(001)$. . . . . 98

$40 \quad \mathrm{LaCoO}_{3}$ Schichtdickenserie . . . . . . . . . . . . . . . . . . . . . . . . . . . . 100

41 Ideal geordnete Doppelperowskitstruktur . . . . . . . . . . . . . . . . . . . . . . . 102

42 Strukturelle und magnetische Daten von $L a_{2} C o M n O_{6}$ auf $\operatorname{STO}(111) \ldots \ldots$

$43 \mathrm{Co} / \mathrm{Mn}$-Abhängigkeit der strukturelle und magnetische Eigenschaften von $\mathrm{La}_{2} \mathrm{CoMnO}_{6} \quad$. . 104

44 TEM-EDX elementspezifische Karten von $\mathrm{La}_{2} \mathrm{CoMnO}_{6} \ldots \ldots \ldots$. . . . . . . . . . . . . . 105

45 HAADF-TEM Karte der Überstruktur-Intensität von $\mathrm{La}_{2} \mathrm{CoMnO}_{6} \ldots \ldots$. . . . . . . . . . . 106

46 EELS-Spektren von B-Platz Kationen in $\mathrm{La}_{2} C o M n O_{6} \ldots \ldots \ldots$. . . . . . . . . . . . . . . . 107

47 Großskalige elementspezifische TEM-EELS Karten von $\mathrm{La}_{2} \mathrm{CoMnO}_{6} \ldots \ldots$. . . . . . . . . . . . 108

48 Strukturelle und magnetische Daten von $\mathrm{La}_{2} \mathrm{CoMnO}$ auf $\mathrm{STO}(111)$ ohne $L a$-Defizit . . . . 110

49 XRD Simulation für den XRD-Ordnungs-Parameter als Funktion der Gittergröße . . . . . . . 113 
50 Model für die Magnetisierung der antiphasen-getrennten Domänen . . . . . . . . . . . . . . . 114

51 Modell der ferrimagnetischen Kopplung in $\mathrm{Sr}_{2} \mathrm{FeMoO}_{6} \ldots \ldots \ldots \ldots$

52 Die temperaturabhängige freie Enthalpien für die Oxidation verschiedener Elemente . . . . . 119

53 Strukturelle und magnetische Daten von $\mathrm{Fe}_{3} \mathrm{O}_{4}$ auf $\mathrm{Al}_{2} \mathrm{O}_{3}(0001)$ und $\mathrm{STO}(011) \quad \ldots \quad \ldots$

54 Ramanspektren von LOMAD Eisenoxidfilmen auf $\mathrm{Al}_{2} \mathrm{O}_{3}(0001) \ldots \ldots$. . . . . . . . . . 123

55 XRD Daten von $S r F e-$ und $S r M o-O x i d e n$ auf $\mathrm{STO}(001) \ldots \ldots \ldots \ldots$

56 XRD Daten von $\mathrm{Sr}_{2} \mathrm{FeMoO}_{6}$ für verschiedene LOMAD Parameter auf STO . . . . . . . . . . 130

57 Magnetische Daten von $\mathrm{Sr}_{2} \mathrm{FeMoO}_{6}$ für verschiedene LOMAD Parameter auf STO . . . . . 132

\section{Tabellenverzeichnis}

1 Strukturelle Daten der LSMO Übergitter . . . . . . . . . . . . . . . . . . . . . . . 81

\section{Abkürzungsverzeichnis}

AFM
AFM-COO
APG
BLT
CMR
DE
DP
EB
FM
FMM
$H$
$M$
MIT
PD
PMI




$\begin{array}{ll}\text { PS } & \text { Phasenseparation/phasensepariert } \\ R & \text { elektrischer Widerstand } \\ \rho & \text { spezifischer elektrischer Widerstand } \\ \text { RMS } & \text { quadratisches Mittel } \\ \text { SE } & \text { Superaustausch } \\ T & \text { Temperatur } \\ \text { TF } & \text { Toleranzfaktor }\end{array}$

\section{Methoden}

EDX

Energiedispersive Röntgenspektroskopie

EELS

Elektronenenergieverlustspektroskopie

HAADF

MAD

High angle annular dark field - Modus

MBE

Metallorganische Aerosol Deposition

MOKE

Molekularstrahlepitaxie

PLD

Magnetooptischer Kerr-Effekt

SFM

Laserstrahlverdampfen

STM

Rasterkraftmikroskopie

TEM

Rastertunnelmikroskopie

$\mathrm{XRD}$

Transmissionselektronenmikroskopie

$\mathrm{XRR}$

Röntgendiffraktion

Röntgenreflexion

\section{Materialien}

DMF

LCMO

LCO

$\mathrm{LMCO}$

LPCMO

LSMO

LSMO:0.5

LMO

$\mathrm{PCMO}$

SFMO

SMO

STO
N,N-Dimethylformamid

$\mathrm{La}_{0.67} \mathrm{Ca}_{0.33} \mathrm{MnO}_{3}$

$\mathrm{LaCoO}_{3}$

$\mathrm{La}_{2} \mathrm{CoMnO}_{6}$

$\left(\mathrm{La}_{1-y} \mathrm{Pr}_{y}\right)_{0.67} \mathrm{Ca}_{0.33} \mathrm{MnO}_{3}$

$\mathrm{La}_{0.67} \mathrm{Sr}_{0.33} \mathrm{MnO}_{3}$

$\mathrm{La}_{0.5} \mathrm{Sr}_{0.5} \mathrm{MnO}_{3}$

$\mathrm{LaMnO}_{3}$

$\mathrm{Pr}_{0.67} \mathrm{Ca}_{0.33} \mathrm{MnO}_{3}$

$\mathrm{Sr}_{2} \mathrm{FeMoO}_{6}$

$\mathrm{SrMnO}_{3}$

$\mathrm{SrTiO}_{3}$ 


\section{Lebenslauf}

Persönliche Daten

Name: Sebastian Hühn

Geburtsdaten: $\quad$ 09.12.1985 in Erfurt

Staatsangehörigkeit: deutsch

Familienstand: ledig

Schulische und Akademische Ausbildung

08/1992 - 06/1996 Grundschule Behringen

08/1996 - 06/2004 Ernst-Abbe-Gymnasium Eisenach

10/2004 - 06/2010 Diplomstudiengang im Fach Physik an der Georg-August-Universität Göttingen

Thema der Abschlussarbeit: „Präparation und Eigenschaften dünner $\left(\mathrm{La}_{1-y} \mathrm{Pr}_{y}\right)_{0.67} \mathrm{Ca}_{0.33} \mathrm{MnO}_{3}$ Schichten "

seit 11/2010 Anfertigung der vorliegenden Dissertation am I. Physikalischen Institut der Georg-August-Universität Göttingen

Berufliche Praxis

04/2008 - 03/2010 Wissenschaftliche Hifskraft an der Georg-August-Universität Göttingen; Übungsgruppenleiter für Physik 1 und Physik 2 für Nebenfächler

11/2010 - 01/2015 Wissenschaftlicher Mitarbeiter im EU FP7 Projekt: IFOX; Übungsgruppenleiter für Festkörper und Materialphysik, Versuchsbetreuer im Masterpraktikum 


\title{
Publikationen
}

\author{
Artikel
}

1. S. Hühn, M. Jungbauer, M. Michelmann, F. Massel, F. Koeth, C. Ballani and V. Moshnyaga, "Modeling of colossal magnetoresistance in $\mathrm{La}_{0.67} \mathrm{Ca}_{0.33} \mathrm{MnO}_{3} / \mathrm{Pr}_{0.67} \mathrm{Ca}_{0.33} \mathrm{MnO}_{3}$ superlattices: Comparison with individual $\left(\mathrm{La}_{1-y} \mathrm{Pr}_{y}\right)_{0.67} \mathrm{Ca}_{0.33} \mathrm{MnO}_{3}$ films", Journal of Applied Physics, 113: $17 \mathrm{D} 701$ 17D701-3, 2013

2. M. Jungbauer, S. Hühn, M. Michelmann, E. Goering and V. Moshnyaga, "Exchange bias in $\mathrm{La}_{0.7} \mathrm{Sr}_{0.3} \mathrm{MnO}_{3} / \mathrm{SrMnO}_{3} / \mathrm{La}_{0.7} \mathrm{Sr}_{0.3} \mathrm{MnO}_{3}$ trilayers", Journal of Applied Physics, 113: $17 \mathrm{D} 709$ 17D709-3, 2013

3. O. Shapoval, S.Hühn, J. Verbeeck, M. Jungbauer, A. Belenchuk and V. Moshnyaga, "Interfacecontrolled magnetism and transport of ultrathin manganite films", Journal of Applied Physics, 113: 17D711 - 17D711-3, 2013

4. V. Moshnyaga, A. Belenchuk, S. Hühn, C. Kalkert, M. Jungbauer, O. I. Lebedev, S. Merten, K.-Y. Choi, P. Lemmens, B. Damaschke, and K. Samwer, "Intrinsic antiferromagnetic coupling underlies colossal magnetoresistance effect: Role of correlated polarons", Phys. Rev. B, 89: 024420, 2014

5. M. Jungbauer, S. Hühn, J.-O. Krisponeit and V. Moshnyaga, "Visible range colossal magnetorefractive effect in $\left(L a_{1-y} P_{y}\right)_{2 / 3} C a_{1 / 3} M n O_{3}$ films", New J. Phys., 16: 063034, 2014

6. M. Foerster, L. Peña, C. A. F. Vaz, J. Heinen, S. Finizio, T. Schulz, A. Bisig, F. Büttner, S.Eisebitt, L. Méchin, S. Hühn, V. Moshnyaga and M. Kläui, "Efficient spin transfer torque in $\mathrm{La}_{2 / 3} \mathrm{Sr}_{1 / 3} \mathrm{MnO}_{3}$ nanostructures", Appl. Phys. Lett., 104: 072410, 2014

7. S. Finizio, M. Foerster, B. Krüger, C. A. F. Vaz, T. Miyawaki, M. A. Mawass, L. Peña, L. Méchin, S. Hühn, V. Moshnyaga, F. Büttner, A. Bisig, L. Le Guyader, S. El Moussaoui, S. Valencia, F. Kronast, S. Eisebitt and M. Kläui, "Domain wall transformations and hopping in $\mathrm{La}_{0.7} \mathrm{Sr}_{0.3} \mathrm{MnO}_{3}$ nanostructures imaged with high resolution x-ray magnetic microscopy", J. Phys.: Condens. Matter, 26: 456003, 2014

8. K. J. O’Shea, D. McGrouther, C. A. Ferguson, M. Jungbauer, S. Hühn, V. Moshnyaga, D. A. MacLaren, "Fabrication of high quality plan-view TEM specimens using the focused ion beam", Micron, 66: 9-15, 2014

9. M. Jungbauer, S. Hühn, R. Egoavil, H. Tan, J. Verbeeck, G. Van Tendeloo, and V. Moshnyaga, "Atomic layer epitaxy of Ruddlesden-Popper $\mathrm{SrO}\left(\mathrm{SrTiO}_{3}\right)_{n}$ films by means of metalorganic aerosol deposition", Applied Physics Letters, 105: 251603, 2014

10. R. Egoavil, S. Hühn, M. Jungbauer, N. Gauquelin, A. Béche, G. van Tendeloo, J. Verbeeck and V. Moshnyaga, "Phase problem in the B-site Ordering of La2CoMnO6: Impact on Structure and Magnetism", Eingereicht in Nanoscale 


\section{Beiträge zu Konferenzen}

- Frühjahrstagung der Deutschen Physikalischen Gesellschaft, Dresden, 2009: Posterpräsentation

- Frühjahrstagung der Deutschen Physikalischen Gesellschaft, Regensburg, 2010: Vortrag

- Frühjahrstagung der Deutschen Physikalischen Gesellschaft, Dresden, 2011: Posterpräsentation

- Frühjahrstagung der Deutschen Physikalischen Gesellschaft, Berlin, 2012: Posterpräsentation

- IFOX summer school, Hesselberg, 2012: Posterpräsentation

- 12th Joint MMM/Intermag Conference, Chicago, USA, 2013: Posterpräsentation

- 24th month IFOX meeting, Enschede, Niederlande, 2013: Vortrag

- Frühjahrstagung der Deutschen Physikalischen Gesellschaft, Regensburg, 2013: Posterpräsentation 



\section{Danksagung}

An dieser Stelle möchte ich allen danken, die mir mein Studium und die Vollendung meiner Promotion ermöglichten:

Meinem Doktorvater Prof. Dr. Vasily Moshnyaga danke ich für die Betreuung meiner Promotion. Er stand mir mit seinem Fachwissen jederzeit zur Seite und wurde nicht zuletzt auch durch private Gespräche zu einem wertvollen und freundschaftlichen Wegbegleiter. Prof. Dr. Christian Jooß und Prof. Dr. Johan Verbeeck bin ich für ihre Zweitgutachten zu Dank verpflichtet. Bei Prof. Dr. Konrad Samwer möchte ich mich bedanken, da er mir die Promotion in seinem Institut ermöglicht hat.

Ganz herzlich möchte ich mich bei meinen Mitdoktoranden und Freunden Markus Jungbauer und Markus Michelmann bedanken, die mich seit den ersten Tagen durch mein Studium begleiten und stark an dem Gelingen dieser Promotion beteiligt waren. Auch der gesamten MAD-Arbeitsgruppe danke ich für die außerordentlich gute Zusammenarbeit und die familiäre Atmosphäre.

Ebenso geht mein Dank an alle weiteren Mitglieder des Institutes für ein konstruktives und kameradschaftliches Arbeitsklima über all die Jahre hinweg. Insbesondere danke ich den Technikern und der Feinmechanik-Werkstatt für die technische Hilfe bei der Anlagen(weiter)entwicklung und Frau Michaela Mecke für ihre Unterstützung bei allen bürokratischen Aufgabenstellungen.

Florian Fischgrabe, Julia Motz und Herma Rabben danke ich für das sorgfältige Korrekturlesen meiner Dissertationsschrift.

Ganz besonders danke ich natürlich meinen Eltern, die mir das Studium erst ermöglichten und mich jederzeit unterstützten, sowie meiner Freundin Julia, ohne Ihre liebevollen Fürsorge, viel Verständnis und Geduld wäre diese Arbeit nicht möglich gewesen.

Für die finanzielle Unterstützung dieser Arbeit im Rahmen des EU FP7 Prokjektes IFOX danke ich der Europäischen Kommission. 
